Gerald Spindler

\title{
Gerichtsnahe Mediation in Niedersachsen
}

Eine juristisch-rechtsökonomische Analyse Abschlussbericht im Auftrag des Niedersächsischen Ministeriums für Justiz und des Niedersächsischen Ministeriums für Wissenschaft und Kultur

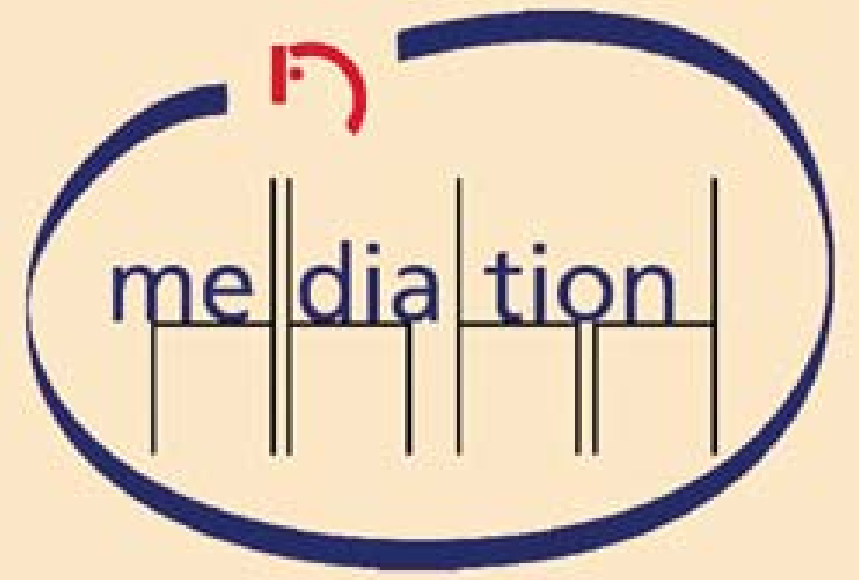



Gerald Spindler

Gerichtsnahe Mediation in Niedersachsen

This work is licensed under the

Creative Commons License 2.0 "by-nd", allowing you to download, distribute and print the document in a few copies for private or educational use, given that the document stays unchanged and the creator is mentioned.

You are not allowed to sell copies of the free version.

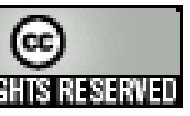


erschienen im Universitätsverlag Göttingen 2006 
Gerald Spindler

\section{Gerichtsnahe Mediation in Niedersachsen}

Eine juristisch-rechtsökonomische Analyse

Abschlussbericht im Auftrag des Niedersächsischen Ministeriums für Justiz und des Niedersächsischen Ministeriums für Wissenschaft und Kultur

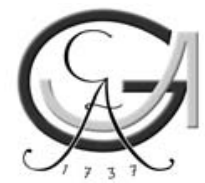

Universitätsverlag Göttingen 2006 
Bibliographische Information der Deutschen Nationalbibliothek

Die Deutsche Nationalbibliothek verzeichnet diese Publikation in der

Deutschen Nationalbibliographie; detaillierte bibliographische Daten sind im Internet über < http://dnb.ddb.de $>$ abrufbar

\author{
Autor \\ Prof. Dr. Dipl.-Oec. Gerald Spindler, Lehrstuhl für Bürgerliches Recht, \\ Handels- und Wirtschaftsrecht, Multimedia- und Telekommunikationsrecht, \\ Rechtsvergleichung, Georg-August-Universität Göttingen, Platz der \\ Göttinger Sieben 6, 37073 Göttingen
}

\author{
Mitwirkende \\ Dipl.-Jur. Dipl.-Vw. Katharina Apel \\ Dr. Judith Volkmann LL. M. \\ Dipl.-Jur. Matthias Mönch
}

\title{
Auftraggeber
}

Niedersächsisches Ministerium für Justiz

Niedersächsisches Ministerium für Wissenschaft und Kultur

Dieses Buch ist auch als freie Onlineversion über die Homepage des Verlags sowie über den OPAC der Niedersächsischen Staats- und

Universitätsbibliothek (http://www.sub.uni-goettingen.de) erreichbar und darf gelesen, heruntergeladen sowie als Privatkopie ausgedruckt werden [Es gelten die Lizenzbestimmungen der Onlineversion]. Es ist nicht gestattet, Kopien oder gedruckte Fassungen der freien Onlineversion zu veräußern.

Satz und Layout: Marc Weber, Jörn Heckmann

Umschlaggestaltung: Kilian Klapp

(C) 2006 Universitätsverlag Göttingen

http://univerlag.uni-goettingen.de

ISBN-10: 3-938616-67-9

ISBN-13: 978-3-938616-67-3 


\section{Inhaltsverzeichnis}

A. Untersuchungsauftrag und Gegenstand der Untersuchung 1

B. Gang der Untersuchung 3

C. Erster Teil: Die Rechtlichen Rahmenbedingungen der gerichtsnahen Mediation

I. Überblick 5

II. Das Projekt „Gerichtsnahe Mediation in Niedersachsen“ 6

III. Modellprojekte zur gerichtsnahen Mediation in anderen Bundesländern

IV. Die rechtliche Zulässigkeit des gerichtlichen Mediationsangebots

1. Richterliches Dienstrecht

2. Zulässigkeit der gerichtsnahen Mediation nach dem Rechtsberatungsgesetz

a) Gerichtsnahe Mediation als nach dem Rechtsberatungsgesetz erlaubnispflichtige Tätigkeit

aa) Die Rolle des Rechts in der Mediation

bb) Schwerpunkt der Mediatorentätigkeit

b) Erlaubnisfreiheit der gerichtsnahen Mediation nach Art. 1 \& 3 Nr. 1 RBerG

3. Verfassungs- und wettbewerbsrechtliche Grenzen

a) Verfassungsrechtliche Grenzen

b) Wettbewerbsrechtliche Grenzen

aa) Das gerichtliche Mediationsangebot als

Wettbewerbshandlung

bb) Wettbewerbswidrigkeit des gerichtlichen

Mediationsangebotes

(1) Unlautere Preisunterbietung 19

(2) Vertrauensmissbrauch 20

(3) Autoritätsmissbrauch 21

(4) Ausnutzung amtlicher Beziehungen zum Wettbewerb

c) Rechtspolitische Handlungsempfehlung 
4. Prozessuale Einbettung des gerichtlichen Mediationsangebots 26

5. Fazit

V. Die rechtlichen Beziehungen zwischen der Justiz und den an der gerichtsnahen Mediation beteiligten Parteien

1. Vertragsrechtliche Beziehungen

a) Vertragsinhalt

b) Qualifizierung als öffentlich-rechtlicher Vertrag?

aa) Gerichtsnahe Mediation im Zivilprozess

bb) Gerichtsnahe Mediation im verwaltungsoder sozialgerichtlichen Verfahren

c) Vertragstypische Einordnung

2. Haftungsfragen

33

3. Fazit

VI. Die rechtliche Beziehung zwischen den an der gerichtsnahen Mediation beteiligten Konfliktparteien

1. Durchsetzbarkeit

a) Materiell-rechtlicher Vergleich

36

aa) Vergleichsschluss bei Mediation im Zivilprozess

36

bb) Zulässigkeits- und Wirksamkeitsgrenzen für den

Vergleichsschluss bei Mediation im verwaltungsoder sozialgerichtlichen Verfahren

(1) Verfassungsrechtliche Vorgaben des Gebots von Vorrang und Vorbehalt des Gesetzes

(2) Gleichbehandlungsgrundsatz

(3) Vertragsformverbote

(4) Zustimmung nicht-beteiligter Dritter gemäß § 58 Abs. 1 VwVfG

(5) Form- und Zuständigkeitsfragen $\quad 40$

(6) Zwischenergebnis 40

b) Vollstreckungstitel 41

a) Zivilprozess 41

(1) Prozessvergleich 41

(2) Andere Vollstreckungstitel 44

bb) Verwaltungs- und sozialgerichtliches Verfahren 44

2. Gefahr von Rechtsverlusten während des Mediationsverfahrens

a) Durch Zeitablauf drohende Rechtsverluste $\quad 45$

aa) Verjährung 
bb) Ablauf von Ausschlussfristen $\quad 46$

(1) Geltendes Recht 46

(2) Rechtspolitische Handlungsempfehlung 47

b) Verlust von mit der Rechtshängigkeit der Sache verbundenen Rechtsvorteilen

48

aa) Materiell-rechtliche Wirkungen der Rechtshängigkeit $\quad 48$

bb) Prozesstaktische Vorteile 48

c) Gefahr der Verschiebung von Rechts- und Vermögenspositionen

3. Vertraulichkeit in der gerichtsnahen Mediation 50

a) Materiell-rechtliche Regelungen zum Schutz der Vertraulichkeit in der Mediation 51

b) Schutz der Vertraulichkeit im nachfolgenden Prozess 51

a) Gesetzliche Bestimmungen 51

(1) Zivilprozess 51

(2) Verwaltungs- und sozialgerichtliches Verfahren 53

bb) Erforderlichkeit, Möglichkeiten und Grenzen einer vertraglichen Regelung zum Schutz der Vertraulichkeit im nachfolgenden Prozess

(1) Zivilprozess

(a) Geltendes Recht

(b) Rechtspolitische Handlungsempfehlung

(2) Verwaltungs- und sozialgerichtliches Verfahren

4. Fazit

VII. Kostenaspekte der gerichtsnahen Mediation $\quad 59$

1. Kosten für Gerichts- und Mediationsverfahren $\quad 59$

a) Gerichts- und Mediationskosten nach geltendem Recht 59

a) Zivilprozess $\quad 59$

bb) Verwaltungsgerichtsverfahren $\quad 60$

cc) Sozialgerichtsverfahren 61

b) Rechtsanwaltskosten 62

$\begin{array}{ll}\text { aa) Gebühren bei gerichtsnaher Mediation } & \\ \text { im Zivilprozess } & 62\end{array}$

$\begin{array}{ll}\text { bb) Gebühren bei gerichtsnaher Mediation } & \\ \text { im verwaltungs-gerichtlichen Verfahren } & 64\end{array}$

cc) Gebühren bei gerichtsnaher Mediation im sozialgerichtlichen Verfahren 
2. Erstattung der Kosten für Gerichts- und Mediationsverfahren 66 a) Erstattung durch den Gegner 66

aa) Zivilprozess 66

(1) Scheitern der Mediationsverhandlungen und Fortführung des gerichtlichen Verfahrens 66

(2) Einigung im Laufe der Mediationsverhandlungen und Protokollierung eines Prozessvergleichs 67

(3) Immaterielle Kosten 68

bb) Verwaltungsgerichtsverfahren $\quad 68$

cc) Sozialgerichtsverfahren 68

b) Kostenerstattung durch die Rechtsschutzversicherung 69

3. Prozess- und Mediationskostenhilfe 71

a) Zivilprozess 71

b) Verwaltungs- und sozialgerichtliches Verfahren 71

D. Zweiter Teil: Rechtsökonomische Analyse 72

I. Überblick 72

$\begin{array}{ll}\text { II. Einleitung } & 72\end{array}$

1. Methodischer Ansatz 72

2. Methodologische Grundlagen $\quad 74$

a) Methodologischer Individualismus $\quad 74$

b) Knappheit der Ressourcen $\quad 75$

c) Verhaltensmodell des Homo oeconomicus 75

III. Theoretisches Modell der Anreizstrukturen 77

1. Anreizstrukturen Richterschaft $\quad 78$

a) Richter als Fallmanager $\quad 78$

a) Richterliche Unabhängigkeit $\quad 78$

bb) Arbeitszeitersparnis $\quad 79$

(1) Konzentration auf wesentliche streitige Verfahren 79

(2) Reduzierung des Arbeitsaufwandes 80

cc) Gefahr eines Kompetenzverlustes 81

dd) Beförderungschancen 82

ee) Ausblick: Rechtspolitische Handlungsempfehlungen $\quad 83$

b) Richtermediatoren 85

aa) Unabhängigkeit und Unparteilichkeit des
Richtermediators

bb) Fehlen finanzieller Anreize 86 
cc) Arbeitszeitersparnis

88

(1) Entscheidung für die Tätigkeit als Richtermediator 88

(2) Ausgestaltung der Richtermediatorentätigkeit 89

dd) Gestaltung des Arbeitsalltags 91

ee) Zufriedenheit mit der beruflichen Tätigkeit 91

ff) Beförderungschancen und Verbesserung der Berufschancen $\quad 92$

gg) Ausblick $\quad 92$

2. Anreizstrukturen Anwälte 93

a) Principal-Agent-Problematik zwischen Anwalt und Mandant

93

a) Das Problem 93

bb) Ansätze zur Überwindung des

Principal-Agent-Problems: 95

b) Gebührenordnung 96

a) Gerichtsnahe Mediation im Zivilprozess 96

bb) Gerichtsnahe Mediation im verwaltungsgerichtlichen Verfahren $\quad 97$

cc) Gerichtsnahe Mediation im sozialgerichtlichen $\begin{array}{ll}\text { Verfahren } & 97\end{array}$

dd) Ergebnis 97

c) Transaktionskostenersparnis 98

d) Berufsständische Regulierungen 98

e) Reputation 99

aa) Reputationsgewinn $\quad 99$

bb) Reputationsverlust 99

f) Arbeitsmarktbedingungen und Gewinn von

g) Fazit 100

3. Anreizstrukturen Parteien 101

a) Kostenersparnis 101

aa) Kosten für Gerichts- und Mediationsverfahren 101

(1) Gerichts- und Mediationskosten nach geltendem Recht 102

(a) Zivilprozess 102

(b) Verwaltungsgerichtsverfahren 102

(c) Sozialgerichtsverfahren 102 
(d) Ergebnis

103

(2) Kostenregelung für die gerichtsnahe

Mediation in der Zukunft

103

bb) Rechtsanwaltskosten 105

cc) Verteilung der Kostenlast 105

(1) Kosten für Gerichts- und Mediationsverfahren 106

(a) Zivilprozess

106

(aa) Scheitern der Mediationsverhandlungen und Fortführung des gerichtlichen Verfahrens

106

(bb) Einigung im Laufe der Mediationsverhandlungen und Protokollierung eines Prozessvergleichs

106

(b) Verwaltungsgerichtsverfahren 106

(c) Sozialgerichtsverfahren 107

(d) Ergebnis 107

(2) Immaterielle Kosten 107

(3) Scheitern der gerichtsnahen Mediation 107

(4) Kostenverteilungsregelungen und

Erfolgsaussichten 108

(5) Kostenanreize in Form von Kostensanktionen bereits im geltenden Recht? 108

(6) Kostenanreize in anderen Verfahren (SGG) 114

dd) Kostenerstattung durch die

Rechtsschutzversicherung 114

ee) Prozess- und Mediationskostenhilfe 115

ff) Zeitlich bedingte finanzielle Anreize 116

(1) Anreize für den Kläger 116

(a) Entwertung eines Prozesserfolges durch eine überlange Verfahrensdauer

(aa) Berücksichtigung der Prozessdauer durch Diskontierung

(bb) Schädigung des Klägers durch Brachliegen seines Kapitals 117

(cc) Durch überlange Verfahrensdauer verursachter Konkurs 117

(dd) Überbrückung von langen Wartezeiten 118 
(b) Gefährdung der materiellen Richtigkeit des Urteils durch lange Verfahrensdauer

(c) Konsequenzen

(2) Anreize für den Beklagten

(a) Kreditgewährung als negativer Anreiz für die Mediation

(b) Zeitgewinn durch den Prozess als negativer Anreiz für die Mediation

(c) Zermürben des Gegners durch die lange Prozessdauer als negativer Anreiz für die Mediation

(d) Gefährdung der materiellen Richtigkeit des Urteils durch lange Verfahrensdauer

gg) Ersparnis sonstiger Kosten

(1) Transaktionskosten

(2) Reputationsbezogene Kosten

(3) Erhalt der Parteibeziehungen

(4) Vermeidung von Folgeprozessen

hh) Abwägung der finanziellen Anreize und rechtspolitische Handlungsempfehlungen

b) Endgültigkeit des Mediationsverfahrens

c) Durchsetzbarkeit des Mediationsergebnisses

d) Vertraulichkeit des Mediationsverfahrens

e) Gefahr von Rechtsverlusten

aa) Durch Zeitablauf drohende Rechtsverluste

(1) Verjährung

(2) Ablauf von Ausschlussfristen

bb) Verlust von mit der Rechtshängigkeit der Sache verbundenen Rechtsvorteilen

(1) Materiell-rechtliche Wirkungen der Rechtshängigkeit

(2) Prozesstaktische Vorteile

cc) Gefahr der Verschiebung von Rechts- und Vermögenspositionen

f) Zufriedenheit mit Verfahren und Ergebnis der Mediation129

a) Mediationsverfahren

bb) Mediationsergebnis

g) Reputation 
h) Obligatorische Mediation 132

i) Fazit 134

4. Anreizstrukturen Rechtsschutzversicherungen 136

a) Status quo 137

b) Ausblick: Angebot eines Versicherungsschutzes für (gerichtsnahe) Mediation und mögliche Probleme 137

a) Grundsätzliche Überlegungen zu einem Versicherungsschutz für (gerichtsnahe) Mediation 137

bb) Probleme 139

(1) Moral hazard/ moralisches Wagnis für die Versicherer 139

(2) Adverse Selection/ Negativauslese 140

cc) Empfohlene Ausgestaltungsmöglichkeiten eines Versicherungsschutzes

E. Dritter Teil: Die empirischen Ergebnisse 144

I. Überblick 144

II. Anreizstrukturen Richterschaft 144

1. Richter als Fallmanager 145

a) Arbeitszeitersparnis 145

aa) Konzentration auf wesentliche streitige Verfahren 145

bb) Reduzierung des Arbeitsaufwandes 146

b) Gefahr eines Kompetenzverlustes 149

2. Richtermediatoren 150

a) Arbeitszeit- und Kostenersparnis $\quad 150$

aa) Zeitersparnis durch Mediation 150

bb) Arbeitsbelastung durch Mediation 153

cc) Erforderlichkeit einer Beweisaufnahme im streitigen Prozess 154

dd) Kostenersparnis in Bezug auf eine etwaige Beweiserhebung 155

ee) Zeitersparnis durch Mediation 155

ff) Arbeitsbelastung 157

gg) Ausbildungskosten 161

b) Zufriedenheit mit der beruflichen Tätigkeit 164

c) Beförderungschancen und Verbesserung der
Berufschancen

III. Anreizstrukturen Anwälte 166

1. Gebührenordnungen 166 
2. Transaktionskostenersparnis

3. Zusammenfassung Gebührenordnung und Transaktionskostenersparnis

4. Reputation

5. Arbeitsmarktbedingungen und Gewinn von zusätzlicher Ausbildung und Erfahrung

6. Ergebnis Anreizstrukturen Anwälte

IV. Anreizstrukturen Parteien

1. Zeitlich bedingte finanzielle Anreize

a) Ersparnis an Arbeits- und Zeitaufwand $\quad 170$

b) Ersparnis an Geld

c) Zeitliche Ersparnis

d) Zeitersparnis nach dem Verfahren

e) Kostenersparnis nach dem Verfahren

f) Ersparnis sonstiger Kosten

aa) Transaktionskosten

bb) Erhalt der Parteibeziehungen

cc) Vermeidung von Folgeprozessen

2. Endgültigkeit des Mediationsverfahrens

3. Durchsetzbarkeit des Mediationsergebnisses

a) Mitsprachemöglichkeiten

b) Konstruktive Mitarbeit

c) Beeinflussung durch den Mediator

d) Erreichen der selbst gesteckten Ziele

e) Gerechtigkeit der Vereinbarung

f) Zufriedenheit mit dem Ergebnis insgesamt

g) Dauerhafte Lösung des Konflikts 192

h) Vereinbarung eingehalten 193

4. Zufriedenheit mit dem Verfahren und Ergebnis der Mediation195

a) Mediationsverfahren

195

b) Mediationsergebnis

F. Vierter Teil: Zusammenfassung und Ausblick

G. Literaturverzeichnis 



\section{A. Untersuchungsauftrag und Gegenstand der Untersuchung}

Alternative Streitbeilegungsformen und insbesondere Mediation haben in den letzten Jahren zunehmend Beachtung und Bedeutung gefunden. Gegenüber den normalen streitigen gerichtlichen Verfahren werden der Mediation zahlreiche Vorteile, insbesondere Kosten- und Zeitersparnisse, Vertraulichkeit, Flexibilität der Verhandlungen und des Ergebnisses sowie Akzeptanz des Ergebnisses durch alle Beteiligten, zugeschrieben. ${ }^{1}$ Vor dem Hintergrund, dass Gerichtsverfahren von den Betroffenen oft als langwierig, aufwändig, teuer, oft auch als unpersönlich, unverständlich und unberechenbar erlebt werden, wird mit dem Modellprojekt „Gerichtsnahe Mediation in Niedersachsen“ seit dem 1. März 2002 erkundet, ob und unter welchen Voraussetzungen es sinnvoll ist, die konsensuale und eigenverantwortliche Streitbeilegung durch gerichtsnahe ${ }^{2}$ Mediation als kostengünstige Alternative zum gerichtlichen Verfahren in das Angebotsspektrum der Justiz aufzunehmen und wie gegebenenfalls eine solche Erweiterung des Angebots der Justiz effektiv organisiert werden kann. In Anknüpfung an $\$ 278$ Abs. 5 S. 2 und 3 ZPO soll Rechtssuchenden auch dann, wenn ein gerichtliches Verfahren bereits eingeleitet wurde, ein anderer Weg der Konfliktlösung als der durch Urteil oder gerichtlichen Vergleich eröffnet werden, sofern auf diese Weise ihr Konflikt interessengerechter und umfassender behandelt werden kann. Gemäß \278 Abs. 5 S. 2 und 3 ZPO kann das Gericht den Parteien eine außergerichtliche Streitbeilegung vorschlagen und bei Einverständnis der Parteien für deren Dauer das Ruhen des gerichtlichen Verfahrens anordnen. Mit der Neufassung des $\ 278$ ZPO des am 1. Januar 2002 in Kraft getretenen ZPO-Reformgesetzes soll die einvernehmliche Streitbeilegung auch im Zivilprozess in noch stärkerem Umfang gefördert werden als bisher. ${ }^{3}$

Ziel des Modellprojektes ist es, die Fähigkeit der Justiz und der Rechtssuchenden zur sinnvollen Konfliktlösung zu verbessern. Mit der Aufnahme von Mediation in

1 S. Duve, Mediation und Vergleich im Prozess, S. 84 ff. zum Potential der Mediation; siehe auch Risse, NJW 2000, 1614, 1618 ff.

2 Im Folgenden werden die Begriffe „gerichtsnahe“ und „gerichtsinterne“ Mediation synonym für das Angebot der niedersächsischen Justiz, Mediation an den Gerichten durch einen Richtermediator durchzuführen, verwendet. Eine Anpassung des Sprachgebrauchs erfolgt nicht, um die bei der Projektkonzeption verwendete Terminologie der gerichtsnahen Mediation weitestgehend beizubehalten.

3 BT-Drucks. 14/4722, S. 62. 
das Angebotsspektrum der Justiz soll die Akzeptanz gerichtlicher Streitbeilegung gefördert, ein Beitrag zur Änderung des Streitverhaltens in der Gesellschaft im Sinne einer Stärkung kooperativer Konfliktbewältigung geleistet sowie systematisches Wissen für die Aus- und Weiterbildung gewonnen und vermittelt werden. Nicht zuletzt soll Mediation als staatliches Angebot neben dem herkömmlichen gerichtlichen Verfahren jedoch auch die Belastung sowohl der Justiz als auch der Rechtssuchenden durch einen Rechtsstreit verringern. Dies gilt zum einen mit Blick auf eine finanzielle Entlastung der Gerichte und der Parteien, angestrebt wird aber auch eine Reduzierung der mit streitigen Gerichtsverfahren verbundenen sozial-emotionalen Belastung der Parteien.

$3 \mathrm{Ob}$ und unter welchen Rahmenbedingungen die Aufnahme von Mediation in das Angebotsspektrum der Justiz zur Erreichung dieser Ziele geeignet ist, sollte im Rahmen des von wissenschaftlicher Forschung begleiteten Modellversuchs geklärt werden. Damit Mediation tatsächlich eine effiziente und effektive Alternative darstellen kann, bedarf es der Akzeptanz der Beteiligten. Ob und unter welchen Rahmenbedingungen dieses Akzeptanz vorliegt und wie gegebenenfalls Faktoren des Mediationseinsatzes geändert werden müssen, wurde durch eine empirisch gestützte Begleitforschung untersucht, die sich ihrerseits auf einen verhaltensbezogenen Forschungsansatz stützt, der die Brücke zwischen Empirie und normativen Aussagen schlagen kann. So sollten gleichzeitig wichtige Fundamente für die weitere Ausformung des Mediationsprozesses und seine Einbettung in die Prozessrechtsordnungen gelegt werden. Denn auch hier ist eine Verrechtlichung zu erwarten, da Mediation nicht völlig losgelöst von allgemeinen verfassungs- und prozessrechtlichen Prinzipien betrachtet werden kann. Mit dieser rechtsökonomischen Begleitforschung wurde die Georg-August-Universität Göttingen, Lehrstuhl für Bürgerliches Recht, Handels- und Wirtschaftsrecht, Multimedia- und Telekommunikationsrecht, Rechtsvergleichung (Prof. Dr. iur. Dipl. Oec. Gerald Spindler) beauftragt. Die sozialwissenschaftliche Forschung wurde durch das arpos-Institut, Hannover, durchgeführt, auf deren Ergebnisse und methodischer Ansatz hier vertiefend verwiesen wird ${ }^{4}$. Die Begleitforschung soll schließlich der Rechtspolitik Entscheidungsgrundlagen dafür liefern, ob und wie die gerichtsnahe Mediation langfristig in das Leistungsangebot der Justiz aufgenommen werden soll, insbesondere welche gesetzlichen Rahmenbedingungen geändert werden müssen und wie die gerichtsnahe Mediation in den Aufbau der Justiz einzubetten ist.

\footnotetext{
${ }^{4}$ Siehe dazu http://www.arpos.de/download/fb5.pdf.
} 
Darüber hinaus wurde auf Wunsch der Lenkungsgruppe des Modellprojekts die rechtsökonomische Begleitforschung um eine Untersuchung des Angebots gerichtsinterner Mediation unter betriebswirtschaftlichen Effizienzaspekten erweitert, um zusätzlich Aussagen über mögliche Kostenersparnisse bzw. das mögliche Kostensenkungspotential durch das Angebot gerichtsinterner Mediation treffen zu können. Für diesen Untersuchungsauftrag liegt ein gesonderter Abschlussbericht vor. Ein unter Effizienzaspekten angestellter Vergleich des herkömmlichen streitigen Verfahrens mit der Verfahrensalternative gerichtsinterner Mediation ergab hier unter Berücksichtigung der grundsätzlichen Wesensunterschiede zwischen streitigem Verfahren und gerichtsinternem Mediationsverfahren und unter Berücksichtigung der aufgrund eines geringen Rücklaufs nur begrenzten Aussagekraft der Erhebung gleichwohl für Streitigkeiten, die ansonsten erstinstanzlich vor den Landgerichten zu verhandeln wären, dass durch das gerichtsinterne Mediationsangebot eine Zeitersparnis in Form von Richterarbeitszeit von ca. 40\% erzielt werden kann. Unter Einbeziehung der amtsgerichtlichen Verfahren ist danach immer noch eine Ersparnis von ca. 20\% für sämtliche erstinstanzliche zivilgerichtliche Verfahren gegeben. ${ }^{5}$

\section{B. Gang der Untersuchung}

Um zu klären, unter welchen Voraussetzungen und strukturellen Rahmenbedingungen es sinnvoll ist, die konsensuale und eigenverantwortliche Streitbeilegung durch gerichtsnahe Mediation tatsächlich als effiziente Alternative zum gerichtlichen Verfahren in das Angebotsspektrum der Justiz aufzunehmen und wie eine solche Erweiterung des Angebots der Justiz effektiv organisiert werden kann, sieht der rechtsökonomische Ansatz der Begleitforschung drei wesentliche Untersuchungsschritte vor:

Nach einem kurzen Überblick über das Projekt „,Gerichtsnahe Mediation in Niedersachsen“ (Rn. 11 ff.) sollen in einem ersten Schritt die rechtlichen Rahmenbedingungen der gerichtsnahen Mediation unter dem geltenden Recht, insbesondere Fragen der Zulässigkeit des gerichtlichen Mediationsangebotes (hierzu Rn. 17 ff.), Aspekte der rechtlichen Beziehung zwischen der Justiz und den Parteien (hierzu Rn. 63 ff.) und zwischen den Parteien (hierzu Rn. 77 ff.) sowie Kostenaspekte der gerichtsnahen Mediation (hierzu Rn. 132 ff.) umfassend analysiert werden.

5 Ausführlich hierzu Spindler, Abschlussbericht zur Ausweitung des Begleitforschungsprojekts „Gerichtsnahe Mediation in Niedersachsen“ - Berücksichtigung betriebswirtschaftlicher Effizienzaspekte - PEBB $\ Y$-analoge Datenerhebung zur Gerichtsnahen Mediation, Februar 2005. 
7 In einem zweiten Schritt sind dann die theoretischen Vorarbeiten zur Klärung der Anreizstrukturen für die an einer Konfliktbewältigung Beteiligten zu leisten: Hier sind die derzeitigen rechtlichen Rahmenbedingungen der gerichtsnahen Mediation und die Anreizstrukturen sämtlicher Konfliktbeteiligten - Richterschaft, Anwälte, Konfliktparteien und Rechtsschutzversicherer - rechtsökonomisch zu analysieren (hierzu Rn. 267 ff.).

8 In einem dritten Schritt wird schließlich anhand der empirischen Feldforschung die tatsächliche Auswirkung der Mediation auf die Akzeptanz der Beteiligten zu untersuchen. Dafür wurden in Zusammenarbeit mit der sozialwissenschaftlichen Forschergruppe Fragebögen entwickelt. Aufbauend auf der im zweiten Schritt erarbeiteten theoretischen Analyse der Anreizstrukturen sollen vor dem Hintergrund der empirischen Ergebnisse rechtspolitische Handlungsempfehlungen formuliert werden. ${ }^{6}$

6 Die empirischen Ergebnisse wurden vom arpos-Institut entwickelt und teilweise aufbereitet. Siehe dazu die Zusammenfassung der Ergebnisse unter http://www.arpos.de/download/fb5.pdf. 


\section{Erster Teil: \\ Die Rechtlichen Rahmenbedingungen der gerichtsna- hen Mediation}

\section{I. Überblick}

Als rechtliche Rahmenbedingungen für die gerichtsnahe Mediation sind zunächst neben dienstrechtlichen Vorschriften des DRiG und dem Rechtsberatungsgesetz (RBerG) auch verfassungs- und wettbewerbsrechtliche Grenzen der Zulässigkeit eines solchen Angebots durch die Justiz zu untersuchen. Das Angebot gerichtsnaher Mediation kann darüber hinaus nicht völlig losgelöst von prozessrechtlichen Prinzipien bestehen, daher muss auch seine prozessuale Einbettung in die Zivilprozessordnung analysiert werden. Rechtliche Rahmenbedingungen werden daneben auch durch die erhobenen Gerichts- und Mediationskosten nach dem GKG sowie durch die Rechtsanwaltskosten des RVG gesetzt. In diesem Zusammenhang müssen auch die prozessualen Regelungen zur Erstattung und Verteilung der anfallenden Kosten berücksichtigt werden.

Auch die zu erwartende europäische Mediationsrichtlinie wird Auswirkungen auf die rechtlichen Rahmenbedingungen der (gerichtsnahen) Mediation haben. Der bisher der Öffentlichkeit zugängliche Vorentwurf der EU-RL ${ }^{7}$ beschränkt sich neben der Beschreibung von Ziel und Definition von Mediation in Art. 1 auf die Möglichkeit des Gerichts, in die Mediation zu verweisen (Art. 3), auf die Aufforderung an die Länder, die Qualität von Mediation durch Kontroll- und Fördermaßnahmen sicherzustellen (Art. 4), auf die Aufforderung an die Länder, die Vollstreckung von Mediationsvereinbarungen zu erleichtern (Art. 5), auf die Vertraulichkeit (Art. 6) und auf die Hemmung oder Unterbrechung der Verjährung (Art. 7). Soweit sich hierdurch für die gerichtsnahe Mediation dennoch zusätzlicher gesetzgeberischer Handlungsbedarf ergeben sollte, wird dieser dann an entsprechender Stelle behandelt.

7 Hierzu Proposal for a Directive of the European Parliament and the Council on certain aspects of Mediation in Civil and Commercial Matters of 22.10.2004; 2004/0251 (COD); COM (2004) 718 final; abrufbar unter:

http://europa.eu.int/eur-lex/lex/LexUriServ/site/en/com/2004/com2004_0718en01.pdf, abgedruckt in: ZKM 2004, 149 und in SchiedsVZ 2005, 41, 42. 


\section{Das Projekt „Gerichtsnahe Mediation in Niedersachsen“}

11 Das Modellprojekt „Gerichtsnahe Mediation in Niedersachsen“ wird vom Niedersächsischen Justizministerium in Zusammenarbeit mit dem Anfang 2002 gegründeten gemeinnützigen Verein Konsens e.V. - Verein zur Förderung der Mediation in Niedersachsen - durchgeführt. An sechs Modellgerichten, den Amtsgerichten Oldenburg und Hildesheim, den Landgerichten Hannover und Göttingen sowie dem Verwaltungs- und dem Sozialgericht Hannover, wird seit Beginn der Praxisphase des Projekts am 1. September 2002 den Parteien eines Rechtsstreits in geeigneten Fällen die Möglichkeit geboten, noch nach Klageerhebung ihren Konflikt mit Hilfe eines Mediators einvernehmlich zu lösen. An den Modellgerichten wurden zu diesem Zweck einzelne Richter als Mediatoren ausgebildet und je nach Größe des Gerichts und Umfang ihrer Mediatorentätigkeit von ihren richterlichen Aufgaben freigestellt. Voraussetzung für die Durchführung eines Mediationsverfahrens ist dabei, dass der Richtermediator selbst nicht als gesetzlicher Richter für den Fall zuständig ist. Der Verein Konsens e.V. beschäftigt zusätzlich seit dem 1. März 2003 eine Psychologin, die schwerpunktmäßig in familienrechtlichen Verfahren als Mediatorin tätig wird.

12 Die gesetzlich zuständigen Richter der Modellgerichte wurden durch entsprechende Schulungen zu sogenannten Fallmanagern qualifiziert und haben die Aufgabe, die Parteien und ihre Anwälte in mediationsgeeigneten Fällen über Mediation sowie das vor Ort bestehende Angebot von Mediation zu informieren und ihnen die gerichtsnahe Mediation vorzuschlagen, wobei die Initiative zur Mediation jedoch auch von den Parteien bzw. ihren Anwälten selbst ausgehen kann. Die Parteien und ihre Anwälte können frei entscheiden, ob sie das Angebot der gerichtsnahen Mediation nutzen oder das gerichtliche Verfahren fortsetzen wollen. Entscheiden sich die Parteien nach der Rechtshängigkeit der Klage für die Mediation, wird das gerichtliche Verfahren für die Dauer der Mediation zum Ruhen gebracht. Ist die Mediation erfolgreich, endet sie mit einer schriftlichen Vereinbarung der Parteien, mit der sie ihren Konflikt einvernehmlich beilegen. Das gerichtliche Verfahren wird beendet, indem die Parteien ihre in der Mediation getroffene Vereinbarung als gerichtlichen Vergleich - vor dem gesetzlich zuständigen Richter - abschließen, übereinstimmende Erledigungserklärungen abgeben oder die Klage zurückgenommen wird. Gelingt es den Parteien nicht, ihren Konflikt in der Mediation einvernehmlich zu lösen, wird das gerichtliche Verfahren vor dem gesetzlichen Richter weitergeführt.

13 Im Hinblick auf die Initiierung des Mediationsverfahrens und die Protokollierung einer in der Mediation getroffenen Vereinbarung als Prozessvergleich, hat sich am 
Landgericht Göttingen im Laufe des Projekts noch eine weitere Vorgehensweise als praktikabel erwiesen, die mittlerweile auch an anderen Projektgerichten angewendet wird: Der gesetzliche Richter gibt die Akten ohne eine gesonderte Prüfung der Mediationseignung des Falles an den Richtermediator ab, der dann direkt den Kontakt zu den Parteien bzw. ihren Anwälten aufnimmt, und zwar unabhängig davon, ob der Fall als typischerweise besonders geeignet erscheint. Als mediationsgeeignet wird nach dieser Praxis vielmehr jeder Fall angesehen, in dem die Parteien noch bereit sind, miteinander zu sprechen. Einigen sich die Parteien in der Mediation, so nimmt teilweise auch der Richtermediator in analoger Anwendung der $\iint 278$ Abs. 5 S. 1, 362 ZPO anstelle des gesetzlich zuständigen Richters direkt im Anschluss an die Mediationsverhandlungen das gerichtliche Verfahren wieder auf und nimmt die von den Parteien getroffene Vereinbarung als Prozessvergleich entgegen. ${ }^{8}$

Die Inanspruchnahme des gerichtlichen Mediationsangebots ist für die Parteien während der Dauer des Projekts kostenlos. Damit soll für die Parteien der Anreiz erhöht werden, sich für die gerichtsnahe Mediation zu entscheiden, und sichergestellt werden, dass Mediation auch in einer lohnenswerten Anzahl von Fällen durchgeführt wird. ${ }^{9}$

\section{Modellprojekte zur gerichtsnahen Mediation in anderen Bundesländern}

Die Integration der Mediation in das gerichtliche Verfahrensangebot wird mittlerweile auch in anderen Bundesländern in verschiedenen Projekten erprobt. Bereits seit dem Jahr 2000 fand am Verwaltungsgericht Berlin ein Modellversuch zur gerichtsnahen Mediation statt, ${ }^{10}$ welcher nach dem Abschlussbericht vom April $2005^{11}$ zur Einführung von Mediationsangeboten auch an den Berliner Zivilgerichten führte. Weitere Projekte zur gerichtsnahen Mediation wurden von BadenWürttemberg ${ }^{12}$, Hessen ${ }^{13}$, Mecklenburg-Vorpommern ${ }^{14}$, Nordrhein-Westphalen ${ }^{15}$ und Sachsen-Anhalt ${ }^{16}$ ins Leben gerufen.

8 Zur Zulässigkeit und Begründung dieser Analogie siehe unten Rn. 89. Näher zur Praxis am Landgericht Göttingen Götz v. Olenbusen, ZKM 2004, 104 f.; Götz v. Olenbusen, DRiZ 2003, 396, 397.

9 Vgl. dazu http://www.mediation-in-niedersachsen.de/Das_Projekt/das_projekt.html.

10 Dazu Ortloff, NVwZ 2004, 385 f., 388; Ortloff NVwZ 2002, 1310, 1316.

${ }^{11}$ Siehe http://www.kammergericht.de/Inhalt\%20Startseite/KG_mediation.htm.

$12 v$. Bargen, DVBl 2004, 468 ff. 
16 Einen etwas anderen Ansatz verfolgt der Modellversuch „Güterichter“, ${ }^{17}$ welches vom Bayerischen Staatsministerium der Justiz seit Anfang 2005 an acht Landgerichten in Bayern durchführt wird. ${ }^{18}$ Ausgehend von der Regelung der Güteverhandlung (\$2 278 Abs. 2 ZPO) wird die Durchführung des Schlichtungsversuchs vom Streitrichter auf einen als Schlichter und Mediator ausgebildeten Richter (sog. Güterichter) verlagert und das Streitgericht von der Güteverhandlung und sonstigen Güteversuchen entlastet. Die Entscheidung über die Einschaltung des Güterrichters liegt im fachlichen Ermessen des Streitrichters. Stimmen die Parteien dem Schlichtungsversuch zu, wird die Sache dem Güterichter formell zugewiesen (\$ 278 Abs. 5 Satz 1 ZPO analog). Im Falle einer Einigung kann der Güterichter einen richterlichen Vergleich beurkunden, womit das Verfahren abgeschlossen ist. Dagegen werden die Akten an den zuständigen Streitrichter zurückgeleitet, sofern der Schlichtungsversuch scheitert.

\section{Die rechtliche Zulässigkeit des gerichtlichen Mediationsangebots}

\section{Richterliches Dienstrecht}

17 Gegen ein gerichtliches Mediationsangebot unter Einsatz von Richtermediatoren können zunächst unter dienstrechtlichen Gesichtspunkten Bedenken bestehen. $\mathrm{Da}$ \4 DRiG dem Richter die Wahrnehmung von Aufgaben außerhalb der originären Rechtsprechungsaufgaben in nur sehr eingeschränktem Umfang gestattet, kommt es für die Beurteilung der dienstrechtlichen Zulässigkeit der hier untersuchten Form der gerichtsnahen Mediation maßgeblich auf die rechtliche Einordnung der richterlichen Mediatorentätigkeit an. Fraglich ist daher, ob die gerichts-

\footnotetext{
${ }^{13}$ Näher zum Projekt „Gerichtsnahe Mediation in der hessischen Verwaltungsgerichtsbarkeit“ $W$ alther, DRiZ 2005, 127 ff.; W alther, ZKM 2005, 53 ff. Zum Mediationsangebot des Landgerichts Frankfurt am Main siehe http://www.lg-frankfurt.justiz.hessen.de.

14 Näher zum Projekt „Gerichtliche Mediation“ Hückstädt, NJ 2005, 289 ff.

15 Siehe http://www.justiz.nrw.de/Online_verfahren_projekte/projekte/modell_owl/Paderborn/Mediati on/index.php.

${ }^{16}$ Siehe http://www.sachsen-anhalt.de/LPSA/index.php?id=mediation.

17 Greger, ZKM 2006, 68 ff. Die Projektbeschreibung und der Zwischenbericht vom Februar 2006 sind abrufbar unter: http://www.jura.uni-erlangen.de/aber/gueterichter.htm.

${ }^{18}$ Greger, ZKM 2006, 68.
} 
nahe Mediation den originären Rechtsprechungsaufgaben zuzuordnen ist, ${ }^{19}$ dem Bereich der Gerichtsverwaltung angehört ${ }^{20}$ oder vielmehr eine neue Form gerichtlicher Tätigkeit darstellt, die weder dem einen noch dem anderen Bereich gerichtlicher Aufgabenerfüllung zuzuordnen ist. ${ }^{21}$

Dabei scheitert die Einordnung der richterlichen Mediatorentätigkeit als originäre Rechtsprechungstätigkeit des Richters von vornherein daran, dass im Rahmen des Mediationsverfahrens eine Entscheidung des Richtermediators in Bezug auf den zu lösenden Konflikt in jedem Fall ausgeschlossen ist. ${ }^{22}$ Denn auch wenn über die Definition des Rechtsprechungsbegriffs im Sinne des Art. 92 GG Uneinigkeit herrscht, so ist man sich dennoch einig, dass Rechtsprechung zumindest durch eine verbindliche, allein am Maßstab des objektiven Rechts getroffene Entscheidung gekennzeichnet ist. ${ }^{23}$ Vor dem Hintergrund, dass auch der Auftrag des Richters gemäß \ 278 Abs. 1 ZPO, in jedem Stadium des gerichtlichen Verfahrens auf eine gütliche Einigung hinzuwirken, ${ }^{24}$ sowie die Güteverhandlung gemäß \ 278 Abs. 2 ZPO als originäre Rechtsprechungsaufgabe des Richters anzusehen sind, 25 wird dagegen eingewandt, dass auch die richterliche Mediatorentätigkeit im Wege der analogen Anwendung des die Güteverhandlung vor einem beauftragten oder ersuchten Richter regelnden $\int 278$ Abs. 5 S. 1 ZPO den originären Rechtsprechungstätigkeiten zugeordnet werden könne. ${ }^{26}$ Hiergegen spricht jedoch, dass die vorgenannten Tätigkeiten im Gegensatz zu der Tätigkeit eines Richtermediators unter Umständen die Vorstufe zur Streitentscheidung darstellen können, was ihre

${ }^{19}$ So Greger, ZKM 2003, 240, 244; v. Bargen, DVBl 2004, 468, 474 f.

${ }^{20}$ So Ortloff, in: Haft/Schlieffen, Handbuch Mediation, S. 762, 788.

${ }^{21}$ In diese Richtung Pitschas, NVwZ 2004, 396, 402; siehe auch Schreiber, Betrifft Justiz 2004, 216, 218.

22 Ausführlich Volkmann, Mediation im Zivilprozess. Rechtliche Rahmenbedingungen für ein gerichtsinternes Mediationsangebot, S. 23 ff.; siehe auch Pitschas, NVwZ 2004, 396, 402; Klose, ZKM 2005, 146, 147. Ebenso für die Tätigkeit des Schlichters in einem dem Prozess vorgeschalteten außergerichtlichen Schlichtungsverfahren Preibisch, Außergerichtliche Vorverfahren in Streitigkeiten der Zivilgerichtsbarkeit, S. 91; Prütting, Außergerichtliche Streitschlichtung, S. 23; Jansen, Die außergerichtliche obligatorische Streitschlichtung nach \$15 a EGZPO, S. 194.

${ }^{23}$ Benda/Weber, ZZP 96 (1983), 285, 287; Wolf, ZZP 99 (1986), 361, 372; Classen, in: v. Mangoldt/Klein/Starck, GG, Band 3, Art. 92 Rn.10; Scbulze-Fielit, in: Dreier, GG, Band 3, Art. 92 Rn. 24 ff. und 42; Schmidt-Räntsch, DRiG, $\ 1$ Rn. 4; siehe auch Dütr, Rechtsstaatlicher Gerichtsschutz im Privatrecht, S. 88 ff.; Preibisch, Außergerichtliche Vorverfahren in Streitigkeiten der Zivilgerichtsbarkeit, S. 89 ff. m.w.N.

${ }^{24}$ Voßkuble, Rechtsschutz gegen Richter, S. 74.

25 Greger, in: Zöller, ZPO, \2 278 Rn. 11; Wrege, DRiZ 2003, 130, 132.

${ }^{26}$ So $v$. Bargen, DVBl 2004, 468, $474 \mathrm{f}$. 
Zuordnung zum Bereich originärer Rechtsprechungsaufgaben rechtfertigt. ${ }^{27}$ Die Tätigkeit des Richtermediators kann dagegen keinerlei Auswirkungen auf eine spätere Streitentscheidung haben. Denn auf Grund der von den Beteiligten regelmäßig getroffenen Vertraulichkeitsabrede kann der Inhalt der Mediationsverhandlungen bei deren Scheitern nicht zum Gegenstand des anhängigen Gerichtsverfahrens gemacht werden. ${ }^{28}$ Eine Zuordnung der richterlichen Mediatorentätigkeit zum Bereich der originären Rechtsprechungsaufgaben des Richters scheidet folglich aus. ${ }^{29}$

19 Gemäß \ 4 Abs. 2 DRiG darf der Richter ausnahmsweise jedoch auch bestimmte Aufgaben außerhalb seiner originären Rechtsprechungsaufgaben wahrnehmen. Dies sind die Aufgaben der Gerichtsverwaltung ( 4 Abs. 2 Nr. 1 DRiG), andere Aufgaben, die auf Grund eines Gesetzes Gerichten oder Richtern zugewiesen sind ( 4 Abs. 2 Nr. 2 DRiG), Aufgaben der Forschung und Lehre an einer wissenschaftlichen Hochschule, öffentlichen Unterrichtsanstalt oder amtlichen Unterrichtseinrichtung ( $\int 4$ Abs. 2 Nr. 3 DRiG), Prüfungsangelegenheiten ( 4 Abs. 2 Nr. 4 DRiG) sowie der Vorsitz in Einigungsstellen und entsprechenden unabhängigen Stellen im Sinne des \104 S. 2 Bundespersonalvertretungsgesetz (BPersVG). Mangels gesetzlicher Vorschrift gemäß $\int 4$ Abs. 2 Nr. 2 DRiG könnte für die gerichtsnahe Mediation allein eine Zuordnung zum Aufgabenbereich der Gerichtsverwaltung gemäß 4 Abs. 2 Nr. 1 DRiG in Betracht kommen. ${ }^{30}$ Zum Bereich der Gerichtsverwaltung gehört die gesamte verwaltende Tätigkeit der Gerichte, die nicht unmittelbar die Erfüllung von Rechtsprechungsaufgaben darstellt, jedoch für diese die unabdingbaren materiellen und personellen Voraussetzungen schaffen muss. ${ }^{31}$ Vor dem Hintergrund, dass auch das gerichtliche Mediationsangebot das Ziel verfolgt, die Justiz zu entlasten und auf diese Weise die Voraussetzungen für eine effektive Erfüllung der den Gerichten zugewiesenen Rechtsprechungsaufgaben zu schaffen, könnte man daher versucht sein, die richterliche Mediatorentätigkeit dem Aufgabenbereich der Gerichtsverwaltung zuzuordnen. Bei genauerem Hinsehen sprechen jedoch sowohl die sachliche Nähe der richterlichen Mediatorentätigkeit zur Aufgabe des Richters in der Güteverhand-

\footnotetext{
27 Siehe dazu Greger, in: Zöller, ZPO, \278 Rn. 11.

28 Volkmann, Mediation im Zivilprozess. Rechtliche Rahmenbedingungen für ein gerichtsinternes Mediationsangebot, S. 26 ff., auch zum folgenden.

${ }^{29}$ Ebenso Prütting, ZKM 2006,100, 101. Nachdrücklich für eine Einordnung als originäre Rechtsprechungsaufgabe dagegen v. Bargen, DVBl 2004, 468, 474 f.; Greger, ZKM 2003, 240, 244.

${ }^{30}$ So Ortloff, in: Haft/v. Schlieffen, Handbuch Mediation, S. 762, 788.

${ }^{31}$ Kissel, GVG, $\ 12$ Rn. 85.
} 
lung als auch das Fehlen vergleichbarer Tätigkeiten in den bekannten Aufgabenbereichen der Gerichtsverwaltung ${ }^{32}$ gegen diese Zuordnung. ${ }^{33}$

Da die Tätigkeit des Richtermediators folglich weder dem Bereich originärer Rechtsprechungsaufgaben noch dem Bereich der Gerichtsverwaltung zuzuordnen ist, ${ }^{34}$ ist für die dienstrechtliche Zulässigkeit der richterlichen Mediatorentätigkeit eine landesrechtliche Regelung im Sinne von $\int 4$ Abs. 2 Nr. 2 DRiG erforderlich, welche dem Richter die Tätigkeit im Rahmen der gerichtsnahen Mediation als ausdrücklich erlaubte Tätigkeit zuweist.

Das Fehlen einer ausdrücklichen Zuweisung hat jedoch keine über das Dienstrecht hinausgehenden Konsequenzen, insbesondere kommen dadurch keine Verfahrensfehler in Betracht, denn der Prozessvergleich wird wiederum vor dem gesetzlichen Richter geschlossen, so dass eine Unwirksamkeit aus prozessualen Gründen nicht gegeben ist. Auch sonstige Gründe für die Unwirksamkeit des Prozessvergleichs kommen nicht in Betracht. So könnte die Wahrnehmung eigentlich nicht vorgesehener richterlicher Aufgaben allein dienstrechtliche Konsequenzen haben, jedoch ist bei dem vorliegenden Angebot der gerichtsnahen Mediation von der niedersächsischen Justiz die Tätigkeit der gerichtsnahen Mediation und somit auch die Richtermediatorentätigkeit gerade vom Dienstherrn gewollt.

\section{Zulässigkeit der gerichtsnahen Mediation nach dem Rechtsberatungsgesetz}

Fraglich ist darüber hinaus, ob die hier untersuchte Form der gerichtsnahen Mediation unter Einsatz von Richtermediatoren nach den Vorgaben des Rechtsberatungsgesetzes zulässig ist. Denn wie vorstehend erörtert, handelt der Richtermediator nicht im Rahmen seiner originären Rechtsprechungsaufgaben.

Die Bundesregierung hat zwar den Entwurf eines neuen Rechtsdienstleistungsgesetzes (RDG) vorgelegt, ${ }^{35}$ dessen $\int 2$ Abs. 3 Nr. 1 von vornherein bestimmt, dass

$32 \mathrm{Zu}$ den klassischen Bereichen der Gerichtsverwaltung gehören die Bereitstellung von Gebäuden sowie aller erforderlichen Arbeitsmittel, die personelle Ausstattung des Gerichts für sämtliche Aufgabenbereiche, die Personalverwaltung einschließlich der Dienstaufsicht, des Haushalts-, Kassen- und Rechnungswesens sowie der Statistik, Aus- und Fortbildung des Nachwuchses, der Ausübung des Hausrechts sowie auch der allgemeinen Rechts- und Amtshilfe durch die Gerichte, siehe dazu Kissel, GVG, \12 Rn. 85; Schmidt-Räntsch, DRiG, \ 4 Rn. 16.

33 Volkmann, Mediation im Zivilprozess. Rechtliche Rahmenbedingungen für ein gerichtsinternes Mediationsangebot, S. 29.

34 So auch Pitschas, NVwZ 2004, 396, 402; ähnlich Schreiber, Betrifft Justiz 2004, $216,218$.

35 Abrufbar unter: http://www.bmj.de/media/archive/746.pdf. 
es sich bei Mediation und jeder vergleichbaren Form der Streitbeilegung nicht um eine Rechtsdienstleistung im Sinne des Gesetzes handelt. Gleichwohl ist zu untersuchen, ob die Tätigkeit des Richtermediators eine nach geltendem Recht zulässige, den Vorgaben des Rechtsberatungsgesetzes entsprechende Tätigkeit darstellt.

\section{a) Gerichtsnahe Mediation als nach dem Rechtsberatungsgesetz erlaubnispflichtige Tätigkeit}

24 Art. 1 S 1 Abs. 1 S. 1 RBerG unterstellt grundsätzlich jede geschäftsmäßige Besorgung fremder Rechtsangelegenheiten der Erlaubnispflicht. ${ }^{36}$ Im Hinblick auf die Zulässigkeit der gerichtsnahen Mediation durch Richter und von der Justiz beauftragte Psychologen kommt es daher zunächst darauf an, ob es sich hierbei um die Besorgung fremder Rechtsangelegenheiten handelt.

$25 \mathrm{Ob}$ es in der Mediation um die Besorgung von Rechtsangelegenheiten und nicht vielmehr um die Besorgung anderer, nämlich wirtschaftlicher, persönlicher oder sozialer Angelegenheiten geht und wie diese voneinander abzugrenzen sind ${ }^{37}$, ist umstritten. Während die Rechtsprechung Mediation in den bislang entschiedenen Fällen als erlaubnispflichtige Rechtsbesorgung eingeordnet hat, ${ }^{38}$ gibt es in der Literatur Meinungsverschiedenheiten über die Frage, ob bzw. unter welchen Voraussetzungen nicht-anwaltliche Mediation als Besorgung fremder Rechtsangelegenheiten im Sinne des Rechtsberatungsgesetzes zu qualifizieren ist. Maßgeblich ist, welche Rolle nach dem Willen der Parteien das Recht im Mediationsverfahren und in der Mediatorentätigkeit spielt. ${ }^{39}$

\section{aa) Die Rolle des Rechts in der Mediation}

26 Zwar wird das Potential der Mediation gerade in der Loslösung von einer rein juristischen Betrachtungsweise gesehen. ${ }^{40}$ Das Recht spielt jedoch auch in der

\footnotetext{
36 Rennen/Caliebe, RBerG, Art. 1 \1 Rn. 5, 8.

${ }^{37}$ Vgl. dazu Haffke, in: Duss-von Werdt/Mähler/Mähler, Mediation: Die andere Scheidung, S. 65, 86 ff.; Glenewinkel, Mediation als außergerichtliches Konfliktlösungsmodell, S. 332 f.

${ }_{38}$ LG Hamburg NJW 2000, 1514; LG Rostock NJW-RR 2001, 1290; OLG Rostock, NJW-RR 2002, 642 jeweils jedoch ohne nähere Auseinandersetzung mit Mediationsverfahren und Mediatorentätigkeit unter dem Gesichtspunkt der Besorgung fremder Rechtsangelegenheiten.

39 Ausführlich zu dieser Frage Volkmann, Mediation im Zivilprozess. Rechtliche Rahmenbedingungen für ein gerichtsinternes Mediationsangebot, S. $31 \mathrm{ff}$.

${ }^{40}$ Risse, BB-Beilage Nr. 9 zu BB 1999, 1, 3.
} 
Mediation eine Rolle, ${ }^{41}$ indem es zum Beispiel den „Wert“ der Handlungsalternativen der Beteiligten bestimmt ${ }^{42}$ und damit den Rahmen für mögliche Einigungsoptionen bzw. den Ausstieg aus den Mediationsverhandlungen absteckt, ${ }^{43}$ den Parteien aber auch als „Schatzkammer" zur Verfügung steht, ${ }^{44}$ aus der sie sich bei der Gestaltung ihrer Einigungsoptionen und zur Verwirklichung von Wertschöpfungspotentialen bedienen können. ${ }^{45}$

\section{bb) Schwerpunkt der Mediatorentätigkeit}

Es stellt sich aber die Frage, ob aufgrund der Bedeutung des Rechts im Mediationsverfahren die Tätigkeit des Mediators grundsätzlich als Besorgung fremder Rechtsangelegenheiten anzusehen is ${ }^{46}$ oder ob die Einordnung der Mediatorentätigkeit als Rechtsbesorgung nicht vielmehr davon abhängt, welche Rolle der Mediator nach dem Willen der Parteien in der Mediation einnimmt. ${ }^{47}$ Teilweise wird in der Literatur die Ansicht vertreten, dass sich eine rechtsbesorgende Tätigkeit im Sinne des Rechtsberatungsgesetzes mit dem Rollenverständnis des Mediators als neutralem Dritten nicht vertrage und schon aus diesem Grund Rechtsberatung in der Mediation nicht stattfinde und auch nicht stattfinden dürfe. ${ }^{48}$ Diese Sichtweise berücksichtigt jedoch nicht, dass der Mediator nicht nur der Neutralität, sondern auch dem Gebot der Fairness verpflichtet ist. Zu den Aufgaben des Mediators gehört es auch, ein faires Ergebnis der Verhandlungen zu gewährleisten ${ }^{49}$ und auf die Einhaltung eines absoluten Mindestmaßes an rechtlicher Ordnung hinzuwirken. ${ }^{50}$ Dies entspricht auch den Vorgaben des von der EU-Kommission in Zu-

${ }^{41}$ Eingehend zur Rolle des Rechts in der Mediation Köper, Die Rolle des Rechts im Mediationsverfahren, S. $76 \mathrm{ff}$.

${ }^{42}$ Eidenmüller, in: Henssler/Koch, Mediation in der Anwaltspraxis, S. 39, 57; Köper, Die Rolle des Rechts im Mediationsverfahren, S. 79; Risse, Beilage Nr. 9 zu BB 1999, 1, 3.

${ }^{43}$ Eidenmüller, in: Breidenbach/Coester-Waltjen/Heß/Nelle/Wolf, Konsensuale Streitbeilegung, S. 45, 47; Risse, Beilage Nr. 9 zu BB 1999, $1,4$.

${ }^{44}$ Mäbler/Mäbler, in: Dieter/Montada/Schulze, Gerechtigkeit im Konfliktmanagement und in der Mediation, S. 9, 32.

${ }^{45}$ Eidenmüller, in: Breidenbach/Coester-Waltjen/Heß/Nelle/Wolf, Konsensuale Streitbeilegung, S. $45,47$.

${ }^{46}$ Hafflee, in: Duss-von Werdt/Mähler/Mähler, Mediation: Die andere Scheidung, S. 65; Heß/Sharma, in: Haft/Schlieffen, Handbuch Mediation, S. 675, 677; Henssler, NJW 2003, 241, 243; Rieble, ZfJ 2001, 13; Mankonski, MDR 2001, 1198, 1199.

${ }^{47}$ Duve, BB 2001, 692, 693; Monßen, AnwBl 2001, 169, 170; Kretschmer, NJW 2003, 1500, 1502.

48 Kretschmer, NJW 2003, 1500, 1502; Monßen, AnwBl 2001, 169, 170.

${ }^{49}$ Hafflee, in: Duss-von Werdt/Mähler/Mähler, Mediation: Die andere Scheidung, S. 65, 67.

${ }^{50}$ Breidenbach, Mediation: Struktur, Chancen und Risiken von Vermittlung im Konflikt, S. 178 f. unter Hinweis auf vertragsrechtliche Institute wie Irrtum, Treu und Glauben oder Sittenwidrigkeit. 
sammenarbeit mit Vertretern verschiedener europäischer Konfliktbeilegungsorganisationen entwickelten europäischen Verhaltenskodexes für Mediatoren: Darin heißt es, dass der Mediator die Parteien aufklären und unter Umständen das Mediationsverfahren für beendet erklären soll, wenn ihm eine von den Parteien getroffene Vereinbarung nicht vollstreckbar oder rechtlich unzulässig erscheint. ${ }^{51} \mathrm{Im}$ Fall eines gravierenden Machtungleichgewichts zwischen den Parteien kann daher eine Einschränkung sowohl der Privatautonomie der Parteien ${ }^{52}$ als auch der Neutralität des Mediators erforderlich sein. ${ }^{53}$ Eine rechtliche Beratung in der Mediation ist auch regelmäßig von den Parteien gewollt. Denn schließlich ist ihnen daran gelegen, dass ihre in der Mediation getroffenen Vereinbarungen im Bereich des rechtlich Zulässigen liegen und tatsächlich alle für die Zukunft relevanten rechtlichen Gesichtspunkte umfassen. ${ }^{54}$ Mediation ist damit als eine nach dem Rechtsberatungsgesetz erlaubnispflichtige Tätigkeit einzuordnen.

\section{b) Erlaubnisfreiheit der gerichtsnahen Mediation nach}

\section{Art. 1 \ 3 Nr. 1 RBerG}

28 Gemäß Art. 1 \ 3 Nr. 1 RBerG wird durch das Rechtsberatungsgesetz jedoch nicht die Rechtsberatung berührt, die von Behörden im Rahmen ihrer Zuständigkeit ausgeübt wird. Für eine Befreiung der gerichtsnahen Mediation von der Erlaubnispflicht kommt es daher darauf an, ob das Angebot gerichtsnaher Mediation in den Zuständigkeitsbereich der Justiz fällt. ${ }^{55}$ Erforderlich, aber auch ausreichend, ist es dafür, dass die Rechtsberatung im Zusammenhang mit dem Aufgabengebiet der Behörde steht. ${ }^{56}$ Eine Zuordnung der gerichtsnahen Mediation zum Aufgabenbereich der Justiz kann sich aus dem mit den Zivilprozessreformen der letzten Jahre verfolgten Anliegen ergeben, die einvernehmliche Streitbeilegung zu fördern und dadurch die Justiz zu entlasten und auf ihre Kernkompetenzen zurückzufüh-

${ }^{51}$ European Code of Conduct for Mediators, 3.2 Fairness of the process, abgedruckt in: ZKM 2004, $148 \mathrm{f}$.

52 Haffke, in: Duss-von Werdt/Mähler/Mähler, Mediation: Die andere Scheidung, S. 65, 67; Köper, Die Rolle des Rechts im Mediationsverfahren, S. 85 f.

53 Breidenbach, Mediation: Struktur, Chancen und Risiken von Vermittlung im Konflikt, S. 174 ff.

54 Henssler, NJW 2003, 241, 243.

55 Vom Behördenbegriff des Rechtsberatungsgesetzes werden nicht nur Behörden im Sinne des $\int 1$ Abs. 4 VwVfG erfasst, sondern auch Gerichte und zwar auch dann, wenn sie als solche und nicht als Justizverwaltungsbehörden tätig sind, Weth, in: Henssler/Prütting, BRAO, Art. 1 \ 3 RBerG Rn. 5; Chemnitz/Johnigk, Rechtsberatungsgesetz, Art. 1 \ 3 Rn. 350; Rennen/Caliebe, Rechtsberatungsgesetz, Art. 1 \ 3 Rn. 2.

56 Chemnitz/Johnigk, Rechtsberatungsgesetz, Art. 1 \& 3 Rn. 354; Rennen/Caliebe, Rechtsberatungsgesetz, Art. 1 S 3 Rn. 8. 
ren. ${ }^{57}$ Das am 1. Januar 2000 in Kraft getretene Gesetz zur Förderung der außergerichtlichen Streitbeilegung ermächtigt die Länder mit der Einführung der in $\ 15$ a EGZPO enthaltenen Öffnungsklausel, zur Entlastung der Justiz ${ }^{58}$ für bestimmte bürgerlich-rechtliche Streitigkeiten ein obligatorisches Güteverfahren vorzuschreiben. In diese Richtung geht auch der durch das am 1. Januar 2002 in Kraft getretene ZPO-Reformgesetz eingeführte $\ 278$ Abs. 5 S. 2 ZPO, der die Möglichkeit einer außergerichtlichen Streitbeilegung auch dann nahe legt, wenn ein Prozess bereits anhängig ist. Aus der amtlichen Begründung zum ZPO-Reformgesetz ergibt sich, dass der Gesetzgeber dabei insbesondere an die Mediation als Methode der außergerichtlichen Streitbeilegung gedacht hat. ${ }^{59}$

Die hier untersuchte Form der gerichtsnahen Mediation gehört damit zwar nicht zu den konkret vom Gesetzgeber vorgegebenen Aufgaben der Justiz. Das gerichtsinterne Mediationsangebot steht jedoch in engem Zusammenhang mit dem Anliegen der Zivilprozessreformen, alternative Konfliktbeilegungsmethoden zu fördern und auf diese Weise die Justiz zu entlasten. Vor diesem Hintergrund ist die gerichtsnahe Mediation dem Zuständigkeitsbereich der Justiz zuzuordnen ${ }^{60}$ und folglich als eine gemäß Art. $1 \rrbracket 3$ Nr. 1 RBerG erlaubnisfreie Tätigkeit einzustufen. Eine begleitende anwaltliche Beratung der Parteien in der Mediation ist mit Blick auf das Rechtsberatungsgesetz daher nicht zwingend erforderlich, d.h. die Parteien können sich ebenso gut für die Alternative der gerichtsnahen Mediation ohne begleitende anwaltliche Beratung entscheiden.

\section{Verfassungs- und wettbewerbsrechtliche Grenzen}

Mediation als Serviceangebot innerhalb der Justiz kann als wirtschaftliche Betätigung des Staates in Konkurrenz zu privaten Mediationsanbietern sowohl wettbewerbsrechtlichen als auch verfassungsrechtlichen Bedenken begegnen.

\section{a) Verfassungsrechtliche Grenzen}

Auch wenn an der grundsätzlichen Berechtigung des Staates, sich wirtschaftlich zu betätigen, keine Zweifel bestehen ${ }^{61}$ und dem Grundgesetz auch kein allgemeiner

\footnotetext{
57 Volkmann, Mediation im Zivilprozess. Rechtliche Rahmenbedingungen für ein gerichtsinternes Mediationsangebot, S. 40 ff., auch zum Folgenden.

58 BT-Drucksache 14/980, S. 5.

59 BT-Drucksache 14/4722, S. 83 f.

60 Ähnlich v. Bargen, DVBl 2004, 468, 475.

${ }^{61}$ Köhler, in: Hefermehl/Köhler/Bornkamm, Wettbewerbsrecht, \4 UWG Rn. 13.6; Emmerich, Der unlautere Wettbewerb der öffentlichen Hand, S. 6; Manssen, in: v. Mangoldt/Klein/Starck, GG,
} 
Subsidiaritätsgrundsatz zu entnehmen ist, nach dem sich die öffentliche Hand erst dann am Wirtschaftsleben beteiligen darf, wenn private Anbieter dazu nicht in der Lage sind, ${ }^{62}$ hat die öffentliche Hand bei ihrer wirtschaftlichen Betätigung gleichwohl die vom Grundgesetz gezogenen Grenzen zu beachten. ${ }^{63}$ Insoweit könnte eine Verletzung der grundrechtlich geschützten Wettbewerbsfreiheit (Art. 2 Abs. 1 GG, Art. 12 Abs. 1 GG, Art. 14 Abs. 1 GG) in Betracht kommen, aber auch des allgemeinen Gleichheitssatzes gemäß Art. 3 Abs. 1 GG. Nach der Rechtsprechung des Bundesverwaltungsgerichts ist in der unternehmerischen Betätigungsfreiheit aus Art. 12 Abs. 1 GG (oder Art. 2 Abs. 1 GG) aber kein subjektives Recht auf Erhaltung und Sicherung eines bestimmten Geschäftsumfangs zu entnehmen. ${ }^{64}$ Vielmehr bedeutet das Hinzutreten des Staates als Konkurrent im Grundsatz lediglich eine systemimmanente Verschärfung des marktwirtschaftlichen Konkurrenzdrucks, ${ }^{65}$ vor der Art. 12 Abs. 1 GG nicht bewahrt, solange die private Konkurrenz nicht unmöglich gemacht wird. ${ }^{66}$ Von einer solchen Konkurrenzsituation ist aber - selbst bei dauerhafter Kostenfreiheit des gerichtlichen Mediationsangebotes - nicht auszugehen.

32 Auch Art. 14 Abs. 1 GG bietet insoweit keinen stärkeren Schutz, da bloße Verdienstmöglichkeiten und Gewinnchancen von der Eigentumsgarantie nicht erfasst werden. ${ }^{67}$ Schließlich ist auch trotz der in aller Regel bestehenden Wettbewerbsvorteile des Staates gegenüber privaten Konkurrenten eine Verletzung des Gleichheitssatzes gemäß Art. 3 Abs. 1 GG nicht zu befürchten, wenn die wirtschaftliche Tätigkeit nur durch einen öffentlichen Zweck sachlich gerechtfertigt ist. ${ }^{68}$ Ein solcher öffentlicher Zweck ist im Fall der gerichtsinternen Mediationangebote neben der Entlastung der Justiz und damit einer Effizienzsteigerung für den Justizapparat auch die Verbesserung der Qualität gerichtlicher Streitbeilegung sowie dadurch auch Ausdruck des günstigen Umgangs mit öffentlichen Ressourcen. Aus

Band 1, Art. 12 Abs. 1 Rn. 79; Piper, GRUR 1986, 574, 575; Stober, ZHR 145 (1981), 565, 578; v.

Gamm, WRP 1984, 303, 305.

62 Piper, GRUR 1986, 574, 575; R. Schmidt, Öffentliches Wirtschaftsrecht - Allgemeiner Teil, S. 520;

Schricker, Wirtschaftliche Tätigkeit der öffentlichen Hand und unlauterer Wettbewerb, S. 41.

${ }^{63}$ Köbler, in: Hefermehl/Köhler/Bornkamm, Wettbewerbsrecht, \ 4 UWG Rn. 13.12; Grupp, ZHR

140 (1976), 367, 379; Isensee, Der Betrieb, 1979, 145, 148.

${ }^{64}$ BVerwGE 71, 183, 193.

65 BVerwGE 71, 183, 193.

${ }^{66}$ BVerwGE 39, 329, 336.

${ }^{67}$ BVerfGE 28, 119, 142; BVerfGE 68, 193, 222 f.; BVerfGE 74, 129, 148.

${ }^{68}$ BVerwGE 39, 329, 337. 
verfassungsrechtlicher Sicht bestehen gegen das Mediationsangebot der Gerichte damit keine Zulässigkeitsbedenken. ${ }^{69}$

\section{b) Wettbewerbsrechtliche Grenzen}

Aus der grundsätzlich unbeschränkten Berechtigung der öffentlichen Hand zur Teilnahme am Wirtschaftsleben folgt die unbeschränkte Anwendbarkeit des Wettbewerbsrechts auf die wirtschaftliche Betätigung der öffentlichen Hand. ${ }^{70}$ Gemäß $\int 3$ des Gesetzes gegen den unlauteren Wettbewerb (UWG) ist wirtschaftliche Betätigung als rechtswidrig zu beurteilen, wenn es sich um unlautere Wettbewerbshandlungen handelt, die geeignet sind, den Wettbewerb zum Nachteil der Mitbewerber, der Verbraucher oder der sonstigen Marktteilnehmer nicht nur unerheblich zu beeinträchtigen.

\section{aa) Das gerichtliche Mediationsangebot als Wettbewerbshandlung}

$\int 2$ Abs. 1 Nr. 1 UWG definiert als Wettbewerbshandlung jede Handlung einer Person mit dem Ziel, zugunsten des eigenen oder eines fremden Unternehmens den Absatz oder den Bezug von Waren oder die Erbringung oder den Bezug von Dienstleistungen zu fördern. Bei einem Tätigwerden der öffentlichen Hand ist daher, soweit sie ausschließlich erwerbswirtschaftliche Zwecke verfolgt, zweifellos auch eine Wettbewerbshandlung gegeben. ${ }^{71}$ Auf der anderen Seite werden rein hoheitliche Tätigkeiten aufgrund ausdrücklicher gesetzlicher Ermächtigung keinesfalls vom Anwendungsbereich des UWG erfasst. ${ }^{72}$ Fraglich ist allein, wann bei solchen Tätigkeiten eine Wettbewerbshandlung anzunehmen ist, mit denen die öffentliche Hand - wie im Fall der gerichtsnahen Mediation - zwar eine öffentliche Aufgabe verfolgt, für die eine ausdrückliche gesetzliche Ermächtigung jedoch nicht existiert. ${ }^{73}$ Abzustellen ist dann darauf, ob die öffentliche Hand im Einzelfall in Wettbewerb zu privaten Anbietern tritt. Darüber hinaus muss die Leistungs-

\footnotetext{
${ }^{69}$ Siehe dazu auch Volkmann, Mediation im Zivilprozess. Rechtliche Rahmenbedingungen für ein gerichtsinternes Mediationsangebot, S. $44 \mathrm{ff}$.

70 Piper, GRUR 1986, 574, 575; Köhler, in: Hefermehl/Köhler/Bornkamm, Wettbewerbsrecht, $\int 4$ UWG Rn. 13.9.

71 Köhler, in: Hefermehl/Köhler/Bornkamm, Wettbewerbsrecht, \4 UWG Rn. 13.18; Keller, in: Harte-Bavendamm/Henning-Bodewig, UWG, \ 2 Rn. 27.

${ }^{72}$ Köhler, in: Hefermehl/Köhler/Bornkamm, Wettbewerbsrecht, \ 4 UWG Rn. 13.21; Keller, in: Harte-Bavendamm/Henning-Bodewig, UWG, \ 2 Rn. 25.

${ }^{73}$ Klose, ZKM 2005, 146, 149. Siehe dazu Köhler, in: Hefermehl/Köhler/Bornkamm, Wettbewerbsrecht, \ 4 UWG Rn. 13.22.
} 
erbringung im Wettbewerb auch von einer entsprechenden subjektiven Zweckrichtung getragen sein. ${ }^{74}$

35 Bei der gerichtsnahen Mediation ist dies zweifellos der Fall. Es handelt sich um ein Angebot gerade in Konkurrenz zu privaten Mediationsanbietern mit der konkreten Zielsetzung, die Justiz am Wettbewerb um die beste Streit- oder Konfliktbeilegung zu beteiligen. ${ }^{75}$ Irrelevant für die Beurteilung ist hier auch, ob tatsächlich bereits private Mediationsanbieter auf dem Markt für gerichtsnahe Mediationsdienstleistungen im Rahmen des $\int 278$ Abs. 5 ZPO tätig sind. Als Mitbewerber i.S.d. $\int 2$ Abs. 1 Nr. 3 UWG ist auch derjenige anzusehen, der in einem potentiellen Wettbewerbsverhältnis steht. ${ }^{76}$ Ein Markteintritt privater Mediationsanbieter in den Markt für außergerichtliche Mediationen im Rahmen des \278 Abs. 5 ZPO ist auch aufgrund niedriger Marktzutrittsschranken jederzeit möglich.

36 Unerheblich ist für die Beurteilung der Abgabe von Waren oder Dienstleistungen durch die öffentliche Hand dagegen, ob sie damit auch eine Gewinnerzielungsabsicht verfolgt. ${ }^{77}$

\section{bb) Wettbewerbswidrigkeit des gerichtlichen Mediationsangebotes}

37 Für die Beurteilung der Wettbewerbswidrigkeit der wirtschaftlichen Betätigung der öffentlichen Hand gilt, dass der Staat, wenn er ohne ausdrückliche gesetzliche Ermächtigung zum konkreten Handeln tätig wird, grundsätzlich denselben Verhaltensanforderungen unterworfen ist, wie jeder andere Marktteilnehmer. ${ }^{78}$ Die öffentliche Hand kann weder eine generelle Vorzugsstellung beanspruchen, wenn sie auch öffentliche Zwecke verfolgt, ${ }^{79}$ noch ist sie generell besonders strengen Verhaltensanforderungen unterworfen. ${ }^{80}$ Doch kann die öffentliche Hand wegen

74 Keller, in: Harte-Bavendamm/Henning-Bodewig, UWG, \ 2 Rn. 28.

75 Ortloff, NVwZ 2002, 1310, 1316; Hoffmann-Riem, JZ 1997, 1, 5 ff.; Ritter, NJW 2001, 3440, 3447; Ziekow, NVwZ 2004, 390, 392; Klose, ZKM 2005, 146, 149; Volkmann, Mediation im Zivilprozess. Rechtliche Rahmenbedingungen für ein gerichtsinternes Mediationsangebot, S. 48 m.w.N.

76 BGH GRUR 2002, 828, 829 - Lottoschein; Köhler, in Hefermehl/Köhler/Bornkamm, Wettbewerbsrecht, $\int 2$ Rn. 71.

77 BGHZ 82, 375, 395 = BGH GRUR 1982, 425, 430; BGH GRUR 1983, 917, 919; Klose, ZKM 2005, 146, 149.

78 Piper, GRUR 1986, 574, 575; Köhler, in: Hefermehl/Köhler/Bornkamm, Wettbewerbsrecht, \ 4 UWG Rn. 13.30 f.; Schünemann, in: Harte-Bavendamm/Henning-Bodewig, UWG, \ 3 Rn. 155 ff.

${ }^{79}$ BGH GRUR 1974, 733; Köhler, in: Hefermehl/Köhler/Bornkamm, Wettbewerbsrecht, \4 UWG Rn. 13.31; Schünemann, in: Harte-Bavendamm/Henning-Bodewig, UWG, \ 3 Rn. 155.

${ }^{80}$ BGH GRUR 1965, 372, 374; BGH GRUR 1973, 530, 531; BGH GRUR 1987, 116, 118; Piper, GRUR 1986, 574, 576; Schünemann, in: Stober/Vogel, Wirtschaftliche Betätigung der öffentlichen 
ihres großen Handlungspotentials schneller in den wettbewerbsrechtlich inkriminierten Bereich geraten als der private Durchschnittsunternehmer. ${ }^{81}$

Die in den $\iint 4$ ff. des neuen UWG beispielhaft geregelten Fallgruppen unlauterer Wettbewerbshandlungen finden auf die hier untersuchte Situation eines kostenfreien Mediationsangebotes durch die Gerichte keine Anwendung. Für die wettbewerbsrechtliche Beurteilung des gerichtlichen Mediationsangebotes ist daher auf die Generalklausel des $\int 3$ UWG zurückzugreifen. Allerdings hatte die Rechtsprechung bereits zur alten Rechtslage und damit für die wettbewerbsrechtliche Beurteilung der Wirtschaftstätigkeit der öffentlichen Hand nach der in \1 UWG a.F enthaltenen Generalklausel eine Reihe von Grundsätzen und Fallgruppen entwickelt, die auch für eine Prüfung der wettbewerbsrechtlichen Zulässigkeit nach \ 3 UWG n.F herangezogen werden können: ${ }^{2}$

\section{(1) Unlautere Preisunterbietung}

Wettbewerbsrechtlich problematisch ist das - kostenlose - gerichtliche Mediationsangebot zunächst unter dem Gesichtspunkt der unlauteren Preisunterbietung. ${ }^{83}$ Eine Preisunterbietung durch die öffentliche Hand kann zwar zulässig sein, wenn sie mit ihrem wirtschaftlichen Tätigwerden zugleich auch eine ihr gesetzlich obliegende Aufgabe wahrnimmt und dabei die Grenzen des sachlich Gebotenen und verfassungsrechtlich Zulässigen ${ }^{84}$ sowie die sachlich berechtigten Interessen privater Wettbewerber berücksichtigt. ${ }^{85}$ Wird die öffentliche Hand dagegen ohne eine gesetzlich zwingende Vorschrift tätig, so ist ein Verstoß gegen das UWG dann anzunehmen, wenn zu befürchten ist, dass der Wettbewerb der öffentlichen Hand die Existenz eines anerkannten Berufsstandes bedroht ${ }^{86}$ und die Wettbewerbs-

Hand, S. 64; Schricker, Wirtschaftliche Tätigkeit der öffentlichen Hand und unlauterer Wettbewerb, S. $136 \mathrm{ff}$.

81 Scbünemann, in: Stober/Vogel, Wirtschaftliche Betätigung der öffentlichen Hand, S. 64 f.; Köbler, in: Hefermehl/Köhler/Bornkamm, Wettbewerbsrecht, \4 UWG Rn. 13.31.

${ }^{82}$ Der Begriff der Wettbewerbshandlung nach $₫ 2$ UWG n.F. entspricht im Zusammenspiel mit der Generalklausel des $₫ 3$ UWG n.F. weitgehend dem Begriff des „Handelns im geschäftlichen Verkehr zu Zwecken des Wettbewerbs“ im Sinne der Generalklausel des \1 UWG a.F. Siehe dazu auch Köhler, in: Hefermehl/Köhler/Bornkamm, Wettbewerbsrecht, \2 UWG Rn. 4.

${ }^{83}$ So auch Klose, ZKM 2005, 146, 149.

${ }^{84}$ BGH GRUR 1993, 917, 919; BGH GRUR 1991, 53, 56.

${ }^{85}$ BGH GRUR 1974, 733, 735; BGH GRUR 1993, 917, 919.

86 BGHZ 82, 375, 396 f. = BGH GRUR 1982, 426, 430; BGH GRUR 1987, 116, 118; Köbler, in: Hefermehl/Köhler/Bornkamm, Wettbewerbsrecht, \4 UWG Rn. 13.35. 
handlung über das $\mathrm{Maß}$ des sachlich gebotenen Verwaltungshandelns hinausgeht. ${ }^{87}$

40 Vor diesem Hintergrund begegnet ein dauerhaft kostenfreies Angebot von gerichtsnaher Mediation erheblichen wettbewerbsrechtlichen Bedenken. ${ }^{88}$ Da private Konkurrenten nicht die Möglichkeit haben, ihre Dienste kostenfrei anzubieten, können sie selbst bei einem gerichtlichen Hinweis auf die Möglichkeit der Inanspruchnahme privater Mediatoren niemals eine echte Alternative zu einem kostenfreien gerichtlichen Mediationsangebot darstellen. Für sie besteht damit die Gefahr, weitgehend vom Mediationsmarkt im Umfeld der Gerichte verdrängt zu werden. Da es sich bei der gerichtsnahen Mediation zudem nicht um eine gesetzlich vorgeschriebene Aufgabe der Justiz handelt, kann eine Wettbewerbswidrigkeit eines auf Dauer kostenfreien Mediationsangebotes durch die Gerichte nicht ausgeschlossen werden. ${ }^{89}$ Dies ändert sich auch nicht durch die Beschränkung der gerichtsinternen Mediation auf bereits bei Gericht anhängigen Verfahren: Dass die Zahl derjenigen Parteien, welche während eines laufenden Gerichtsverfahrens von der Möglichkeit gerichtsexterner Mediation Gebrauch machen, empirisch betrachtet nicht signifikant ist, ${ }^{90}$ lässt allenfalls bei punktueller Betrachtung der gegenwärtigen Situation die Wettbewerbswidrigkeit entfallen. ${ }^{91}$ Für eine dauerhafte Einführung der gerichtsnahen Mediation wäre es daher geboten, eine angemessene Kostenregelung für das gerichtliche Mediationsangebot zu schaffen. ${ }^{92}$

\section{(2) Vertrauensmissbrauch}

41 Von einem wettbewerbswidrigen Handeln ist auch dann auszugehen, wenn die öffentliche Hand das Vertrauen der Bevölkerung missbraucht, um eigenen oder

${ }^{87}$ BGH GRUR 1993, 917, 919; Köbler, in: Hefermehl/Köhler/Bornkamm, Wettbewerbsrecht, $\ 4$ UWG Rn. 13.35; Köbler, in: Köhler/Piper, UWG, $\$ 1$ Rn. 564; Omsels, in: Harte-

Bavendamm/Henning-Bodewig, UWG, \4 Rn. 293.

88 Volkmann, Mediation im Zivilprozess. Rechtliche Rahmenbedingungen für ein gerichtsinternes Mediationsangebot, S. 52 f., auch zum Folgenden.

${ }^{89}$ Ein bloß zeitweilig wettbewerbsbeeinträchtigendes Verhalten führt dagegen gewöhnlich nicht zu einer Gefahr für den Bestand des Wettbewerbs und ist wettbewerbsrechtlich daher auch nicht zu beanstanden, siehe BGH GRUR 1960, 331; BGH GRUR 1979, 321, 322 I; Köbler, in: Köhler/Pieper, UWG, \1 Rn. 492. Die bloß zeitweilige Kostenfreiheit des Mediationsangebotes während der begrenzten Dauer eines Pilotprojektes kann daher noch als wettbewerbsrechtlich zulässig angesehen werden. siehe dazu auch Volkmann, Mediation im Zivilprozess. Rechtliche Rahmenbedingungen für ein gerichtsinternes Mediationsangebot, S. 53.

${ }^{90}$ Greger, ZKM 2003, 240, 242 f.

91 Anders Klose, ZKM 2005, 146, 149 f.

92 Näher dazu unter Rn. 193. 
fremden Wettbewerb zu fördern. ${ }^{93}$ Ein solcher Vertrauensmissbrauch kann bereits in der bloßen Empfehlung eigener oder fremder Leistungen liegen, ${ }^{94}$ wobei Empfehlungen, die im Aufgabenbereich der öffentlichen Hand liegen ${ }^{95}$ und neutral, objektiv und sachgerecht erfolgen, jedoch nicht zu beanstanden sind. ${ }^{96}$ Sofern bei der Empfehlung der eigenen Leistung zumindest auch auf das Angebot privater Anbieter hingewiesen wird, ${ }^{97}$ können auch wertende Beurteilungen noch als wettbewerbsrechtlich zulässig zu beurteilen sein. ${ }^{98}$

Demnach ist es nicht von vornherein als wettbewerbsrechtlich unzulässig zu beurteilen, wenn der zuständige Richter oder der Richtermediator den Parteien vorrangig die Inanspruchnahme des gerichtlichen Mediationsangebotes empfiehlt. ${ }^{99}$ Denn, wie vorstehend unter B. II. 2. festgestellt, gehört das gerichtliche Mediationsangebot zum Zuständigkeitsbereich der Justiz. Solange die Qualität des gerichtlichen Mediationsangebotes sicher gestellt ist, ist eine solche Empfehlung auch nicht unsachgemäß. Voraussetzung für die wettbewerbsrechtliche Unbedenklichkeit einer Empfehlung des eigenen Mediationsangebots durch die Gerichte ist jedoch, dass die Empfehlung stets auch unter Hinweis auf die Möglichkeit der Inanspruchnahme privater Mediatoren erfolgt. ${ }^{100}$

\section{(3) Autoritätsmissbrauch}

Wettbewerbswidriges Handeln der öffentlichen Hand kann darüber hinaus auch unter dem Aspekt des Autoritätsmissbrauchs vorliegen, was etwa bei einer sachwidrigen Druckausübung auf die Kundenentscheidung der Fall wäre. ${ }^{101}$ In einem solchen Fall reicht es aus, wenn die angesprochenen Personen den Eindruck ge-

\footnotetext{
93 OLG Köln NJW-RR 1996, 1448; Köhler, in: Hefermehl/Köhler/Bornkamm, Wettbewerbsrecht, \ 4 UWG Rn. 13.36; Köhler, in: Köhler/Pieper, UWG, \ 1 Rn. 565; Schricker, Wirtschaftliche Tätigkeit der öffentlichen Hand und unlauterer Wettbewerb, S. $181 \mathrm{ff}$.

94 BGH GRUR 1984, 665, 666; Köhler, in: Köhler/Pieper, UWG, \ 1 Rn. 565, 568.

95 BGH GRUR 1984, 665, 666; Köhler, in: Köhler/Pieper, UWG, \ 1 Rn. 565.

${ }^{6}$ Köhler, in: Köhler/Pieper, UWG, \ 1 Rn. 568.

97 BGH GRUR 1987, 119.

98 BGHZ 19, 299, 306; Köhler, in: Hefermehl/Köhler/Bornkamm, Wettbewerbsrecht, \ 4 UWG Rn. 13.39; Köhler, in: Köhler/Pieper, UWG, \1 Rn. 568.

99 Volkmann, Mediation im Zivilprozess. Rechtliche Rahmenbedingungen für ein gerichtsinternes Mediationsangebot, S. 54.

100 Volkemann, Mediation im Zivilprozess. Rechtliche Rahmenbedingungen für ein gerichtsinternes Mediationsangebot, S. 54.

101 BGH GRUR 1964, 210, 213; Köhler, in: Hefermehl/Köhler/Bornkamm, Wettbewerbsrecht, \ 4 UWG Rn. 13.42; Köhler, in: Köhler/Pieper, UWG, \ 1 Rn. 571; Schricker, Wirtschaftliche Tätigkeit der öffentlichen Hand und unlauterer Wettbewerb, S. 177 ff.
} 
winnen, ihre Entscheidung könne wiederum die Entscheidungen des Amtsinhabers beeinflussen. ${ }^{102}$

44 Wettbewerbswidrigkeit des gerichtlichen Mediationsangebotes ist unter diesem Aspekt jedoch nicht zu befürchten: Das gerichtliche Mediationsangebot folgt dem Grundsatz der Freiwilligkeit. Die Parteien können stets frei entscheiden, ob sie das Angebot in Anspruch nehmen wollen oder nicht. Wird diese Freiwilligkeit des Mediationsverfahrens während des Informationsgesprächs zur gerichtsnahen Mediation deutlich zum Ausdruck gebracht, so ist nicht zu befürchten, dass sich die Beteiligten irgendeinem Druck zur Teilnahme an einer Mediation ausgesetzt sehen. ${ }^{103}$

\section{(4) Ausnutzung amtlicher Beziehungen zum Wettbewerb}

45 Wettbewerbswidriges Verhalten der öffentlichen Hand kann schließlich auch gegeben sein, wenn sie ihre amtlichen Beziehungen zum Wettbewerb missbräuchlich ausnutzt, um sich oder anderen wettbewerbliche Vorteile zu verschaffen, also amtliche und erwerbswirtschaftliche Interessen verquickt. ${ }^{104} \mathrm{Im}$ Zusammenhang mit der gerichtsnahen Mediation ist insbesondere die Fallgruppe der Ausnutzung öffentlicher Ressourcen relevant. Dabei ist zu beachten, dass es der öffentlichen Hand wegen ihrer grundsätzlichen Berechtigung zur wirtschaftlichen Betätigung nicht grundsätzlich untersagt ist, bei der Teilnahme am Wettbewerb auf die ihr zur Verfügung stehenden Mittel (Sachmittel, Geldmittel und Personal) im erforderlichen Umfang und in angemessener Weise zurückzugreifen. ${ }^{105}$ Soweit sie mittels der privatwirtschaftlichen Betätigung dazu beitragen möchte, ihre öffentlichen Aufgaben rascher und rationeller zu erfüllen, muss sie aber so vorgehen, dass die Belange privater Anbieter so wenig wie möglich beeinträchtigt werden. ${ }^{106}$ In diesem Zusammenhang ist es beispielsweise als zulässig erachtet worden, dass eine Gemeinde den Verkauf von Kfz-Schildern betreibt, um den Bürgern das Verfahren zu erleichtern, sofern sie gleichzeitig auf private Bezugsmöglichkeiten hinweist. $^{107}$

\footnotetext{
102 BGH GRUR 1984, 665, 667; Köhler, in: Köhler/Pieper, UWG, \1 Rn. 571.

103 Volkmann, Mediation im Zivilprozess. Rechtliche Rahmenbedingungen für ein gerichtsinternes

Mediationsangebot, S. 54.

104 BGH WRP 1999, 650, 654.

105 BGH GRUR 1987, 1167, 118; BGH WRP 1998, 857, 859; Köhler, in: Köhler/Pieper, UWG, \} 1

Rn. 576; Piper, GRUR 1986, 574, 578.

106 BGH GRUR 1974, 733, 734.

107 BGH GRUR 1974, 733, 734.
} 
Zur Vermeidung einer wettbewerbsrechtlich problematischen Konkurrenzsituation ist es beim Angebot der gerichtsnahen Mediation, für das die Justiz auf die ihr zur Verfügung stehenden öffentlichen Ressourcen zurückgreift, daher auch unter dem Aspekt der Ausnutzung amtlicher Beziehungen zum Wettbewerb von Bedeutung, dass die Gerichte in dem Informationsgespräch zur Mediation auch auf die Möglichkeit der Inanspruchnahme privater Anbieter hinweisen. ${ }^{108}$

Wettbewerbsverzerrungen können sich aber auch aus anderen Aspekten heraus ergeben: So könnten Parteien, die sich für eine Mediation entschieden haben, den Weg der gerichtsnahen Mediation beschreiten, weil ihnen hier nicht ein Privater sei dieser auch noch so gut ausgebildet - sondern ein Richter als Mediator zur Seite steht. Hintergrund einer solchen Überlegung mag sein, dass Parteien eine gewisse Vorstellung von einem Richter haben; er genießt bei ihnen alleine schon durch seine staatliche Anstellung, seiner durch sein Amt geforderten Neutralität und die für die Einstellung in den öffentlichen Dienst zu erfüllenden Voraussetzungen eine hohe Reputation, sodass die gerichtsnahe Mediation den Parteien als besonders vertrauenswürdig erscheinen könnte. ${ }^{109}$ In diesem Zusammenhang wurde von Prof. Dr. Greger im Auftrag des Bayerischen Staatsministeriums der Justiz eine Untersuchung hinsichtlich der Entwicklung der außergerichtlichen Schlichtung in Bayern seit Inkrafttreten des Bayrischen Schlichtungsgesetzes durchgeführt, welche insbesondere auf die Schlichtungs-Infrastruktur, die Erfahrungen mit dem Schlichtungsverfahren, die Auswirkungen auf die Gerichte, die Fragen de Zielerreichung und der Bewährung des Regelungskonzepts sowie darauf eingeht, welche Maßnahmen sich empfehlen, um außergerichtlicher Schlichtung zu größerer Wirksamkeit zu verhelfen und den Schlichtungsgedanken im allgemeinen Rechtsbewusstsein nachhaltiger zu verankern. Zum Zweck der Evaluierung und Auswertung von Erfahrungswerten im Kontext einer freiwilligen Inanspruchnahme von Schlichtungsverfahren initiierte das Bayerische Justizministerium darüber hinaus einen Modellversuch zur Förderung der außergerichtlichen Beilegung von Rechtkonflikten (a. be. r.) in Nürnberg, dessen Erfahrungen in die Empfehlungen des Abschlussberichts des Forschungsprojekts ,außergerichtliche Streitbeilegung in Bayern" eingingen. Dieser kommt zu dem Ergebnis, dass sich privat durchgeführte, gerichtsexterne Mediation mit den Problemen eines zersplitterten und lückenhaften Angebots der außergerichtlichen Streitbeilegung konfrontiert sieht und eine Sicherung von Qualitätsstandards bislang weitgehend fehlt. ${ }^{110}$

\footnotetext{
108 Volkmann, Mediation im Zivilprozess. Rechtliche Rahmenbedingungen für ein gerichtsinternes Mediationsangebot, S. 55.

109 So ausweislich Walther, ZKM 2005, 53, 56.

110 Siehe hierzu auch Abschlussbericht a.be.r, S. 102 f.
} 
Dies resultiert schon aus der Tatsache, dass es bislang keinen festgelegten Begriff des Mediators gibt, sondern dass jeder, der mediieren möchte, sich als Mediator bezeichnen kann, unabhängig davon, ob er eine entsprechende Ausbildung absolviert oder gar durch eine Prüfung abgeschlossen hat. Auch von europarechtlicher Seite wird man hier keine Änderungen erwarten können, wie sich aus dem Vorschlag der Kommission für die Mediationsrichtlinie ergibt. Hier wird ausdrücklich angeführt, dass die vorgeschlagene Richtlinie keinerlei Vorschriften über Mediationsverfahren selbst, aber auch nicht über die Benennung oder Zulassung von Mediatoren enthält, sondern dass die hierzu einschlägigen Vorschriften mit Bedacht offen formuliert wurden, um den Mitgliedsstaaten einen möglichst weiten Spielraum zu geben. ${ }^{111}$ Insofern bleibt es hier dem deutschen Gesetzgeber überlassen, eine Qualtitätskontrolle für den Zugang zum Mediatorenberuf per Gesetz zu etablieren. ${ }^{112}$

48 Um die genannten Wettbewerbsverzerrungen zu beseitigen, wäre es empfehlenswert, eine Art Gütesiegel einzuführen, wie dies in Österreich durch das österreichische Zivilrechts-Mediations-Gesetz (ZivMediatG) ${ }^{113}$ geschehen ist. Das Gesetz regelt für das Land Österreich die Einrichtung eines Beirats für Mediation, die Voraussetzungen und das Verfahren für die Eintragung von Personen in die Liste der eingetragenen Mediatoren, die Führung dieser Liste, die Voraussetzungen und das Verfahren für die Eintragung von Ausbildungseinrichtungen und Lehrgängen für Mediation in Zivilrechtssachen, die Führung der hierzu gehörenden Liste, die Rechte und Pflichten der eingetragenen Mediatoren sowie die Hemmung von Fristen durch die Mediation in Zivilsachen. In den $\iint 8 \mathrm{ff}$. ZivMediatG ist vorgesehen, ${ }^{114}$ dass bei dem Bundesminister der Justiz eine Liste der Mediatoren zu führen ist, wobei nur in diese Liste aufzunehmen ist, wer unter anderem fachlich qualifiziert ist. Eine solche fachliche Qualifikation wird in Österreich nur angenommen, wenn die entsprechende Person aufgrund einer entsprechenden Ausbildung über Kenntnisse und Fertigkeiten der Mediation verfügt, hierzu gehören unter anderem Grundlagen in rechtlichen sowie psychosozialen Aspekten. In

\footnotetext{
111 Siehe hierzu Proposal for a Directive of the European Parliament and the Council on certain aspects of Mediation in Civil and Commercial Matters of 22.10.2004; 2004/0251 (COD); COM (2004) 718 final (siehe oben Fn. 7).

112 Ähnlich auch Pitkowitr, SchiedsVZ 2005, 81, 84, der darauf verweist, dass die Ausbildungsrichtlinien des Bundesverbandes Mediation e.V., des Fordervereins Umweltmediation e.V., der deutschen Gesellschaft für Mediation in der Wirtschaft und des Bundesverbandes Mediation in Wirtschaft und Arbeitswelt als Standard ausreichend sein könnten.

113 Österreichisches BGBl. I, 2003, S. 123.

114 Siehe zu den Voraussetzungen für die Anerkennung als Mediator http://www.mediatorenliste.justiz.gv.at/mediatoren/mediatoren.nsf/docs/home.
} 
Deutschland fände eine solche Liste ihr Vorbild beispielsweise in der Erfassung gerichtlicher Sachverständiger durch die Industrie- und Handelskammern..Eine andere Möglichkeit besteht darin, in Übereinstimmung mit dem Wortlaut des Art. 4 des Entwurfs einer Mediationsrichtlinie ${ }^{115}$ auf den Markt als Selbstregulativinstrument zu vertrauen und lediglich freiwillig verpflichtende Kodizes zu ermöglichen. ${ }^{116}$ Eine solche Steuerung erfolgt beispielsweise in den Niederlanden auf verbandsrechtlicher Ebene durch das Nederlands Meetinstituut (NMI), ${ }^{117}$ welches Mediatoren akkreditiert, die die vom NMI definierten Voraussetzungen erfüllen. ${ }^{118}$

Den Status, den ein Richter alleine schon durch seine Stellung als solche innehat und das Vertrauen, dass er dadurch genießt, wird zwar unter Umständen für einen Privaten auch nicht durch ein staatliches Gütesiegel erreicht werden können, aber zumindest können damit gewisse Qualitätsstandards gewährleistet werden, die das Vertrauen in private Mediatoren stärken und die Kluft zwischen dem Vertrauen in Richtermediatoren und private Mediatoren verkleinern helfen.

\section{c) Rechtspolitische Handlungsempfehlung}

Die vorstehenden wettbewerbsrechtlichen Bedenken eines kostenfreien Mediationsangebotes der Gerichte könnten durch eine gesetzliche Verankerung der Mediation im Rahmen des $\int 278$ ZPO ausgeräumt werden. Bei der Entscheidung für die langfristige Aufnahme der Mediation in das Leistungsangebot der Justiz ist eine Änderung der ZPO daher dringend zu empfehlen.

Im Rahmen des $\int 278$ ZPO sollte daher die explizite Nennung der gerichtsinternen Mediation erfolgen. Die Möglichkeit der Verweisung eines Falles an den Richtermediator zur gerichtsinternen Mediation kann entsprechend des bisherigen $\$ 278 Abs. 5 ZPO formuliert werden. Ein ähnlicher Vorschlag findet sich im bayrischen Entwurf eines Gesetzes zur Stärkung der gütlichen Streitbeilegung im Zivilprozess $^{119}$, der eine Neufassung des $\int 278$ Abs. 5 ZPO vorsieht und im Wortlauf

\footnotetext{
115 Hierzu Proposal for a Directive of the European Parliament and the Council on certain aspects of Mediation in Civil and Commercial Matters of 22.10.2004; 2004/0251 (COD); COM (2004) 718 final; abrufbar unter: http://europa.eu.int/eur-lex/lex/LexUriServ/site/en/com/2004/com2004_0718en01.pdf.

116 Die österreichische Lösung wird hingegen als vom Ermessensspielraum der Richtlinie gedeckte (potentielle) Umsetzung anerkannt, vgl. Roth, IDR 2005, 114, 118 f.; Pitkowitr, ZKM 2005, 68, 69.

117 Nederlands Meetinstituut, http://www.nmi.nl.

118 Das niederländische Justizministerium hat jedoch bebereits angekündigt, die Mediatorentätigkeit künftig staatlich zu regulieren, Kilian, ZKM 2005, 186, 187.

119 Bundesrat-Drucks.747/04; auch abrufbar unter: http://www2.justiz.bayern.de/daten/pdf/GesEntwurfStreitbeilegungZivil.pdf.
} 
wie folgt lautet: „Für die Güteverhandlung oder für einen sonstigen Güteversuch kann das Gericht die Parteien vor einen nach der Geschäftsverteilung hierfür zuständigen Richter (Güterichter) oder vor einen beauftragten oder ersuchten Richter verweisen. Hält der Güterichter einen Güteversuch für aussichtslos oder kommt eine Einigung der Parteien in den Fällen des Satzes 1 nicht zu Stande, so wird das Verfahren vor dem Prozessgericht fortgesetzt". Nach dem bayrischen Entwurf wird vom Güterichter auch der Richtermediator, vom Güterversuch auch die gerichtsinterne Mediation erfasst. Durch die Wahl „Güteversuch“ soll allerdings eine zu enge Festlegung auf bestimmte Streitschlichtungsverfahren vermieden werden. Wichtig muss hier sein, dass es sich um einen Richter handelt, der hauptamtlich durch die gerichtliche Geschäftsverteilung mit der Durchführung von Güteverhandlungen und sonstigen Güteversuchen betraut und hierfür in geeigneten Konfliktlösungsmethoden, insbesondere Mediation speziell geschult ist. Der Entwurf will die letzte Entscheidung, Richtermediatoren oder Güterichter bei einem bestimmten Gericht zu schaffen, der richterlichen Geschäftsverteilungsautonomie unterstellen, die im Einzelfall dann von den Präsidien gem. $\ 21 \mathrm{e}$ GVG im Wege der Geschäftsverteilung zu regeln ist.

52 Die bisher in $₫ 278$ Abs. 5 Satz 2 und 3 ZPO enthaltene Möglichkeit des Vorschlags einer außergerichtlichen Streitschlichtung sollte beibehalten werden, so dass letztlich den Parteien die Wahl des Konfliktlösungsangebots überlassen bleiben sollte und Wettbewerb so gewährleistet wird.

53 Wird auf eine solche Gesetzesänderung verzichtet, muss die Ausgestaltung der einzuführenden Gebühren den eben aufgeführten wettbewerbsrechtlichen Bedenken Rechnung tragen und sollte sich daher an den Gebühren privatwirtschaftlich tätiger Mediatoren orientieren. ${ }^{120}$

\section{Prozessuale Einbettung des gerichtlichen Mediationsangebots}

54 Für den Zivilprozess ist die gerichtliche Empfehlung einer außergerichtlichen Streitbeilegung sowie die Aussetzung des gerichtlichen Verfahrens gemäß \251 ZPO zum Zweck der Durchführung einer solchen ausdrücklich in $₫ 278$ Abs. 5 S. 2 und 3 ZPO vorgesehen. ${ }^{121} \mathrm{Ob}$ dabei für den Versuch der einvernehmlichen Streitbeilegung ein gerichtliches oder - wie in $\$ 278$ Abs. 5 S. 2 ZPO vorgesehen ein außergerichtliches Mediationsangebot genutzt wird, ist in prozessrechtlicher Hinsicht unerheblich. Denn auch die Inanspruchnahme eines gerichtsinternen Mediationsangebotes findet außerhalb des streitigen Gerichtsverfahrens statt und

\footnotetext{
$120 \mathrm{Zu}$ möglichen unterschiedlichen Gebührenmodellen siehe unten Rn. 273.

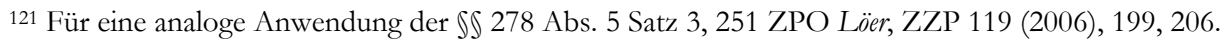


stellt - verfahrensrechtlich - damit den Versuch einer außergerichtlichen Streitbeilegung im Sinne von $₫ 278$ Abs. 5 S. 2 ZPO dar ${ }^{122}$.

Fraglich ist jedoch, ob auch die Verwaltungsgerichtsordnung (VwGO) und das Sozialgerichtsgesetz (SGG) die Durchführung einer Mediation während des gerichtlichen Verfahrens zulassen. Eine dem \278 Abs. 5 S. 2 und 3 ZPO entsprechende Regelung ist in den Prozessordnungen jedenfalls nicht enthalten.

Zu denken ist daher zunächst an die $\iint 173$ VwGO, 202 SGG, welche die Regelungen der ZPO auch im verwaltungs- und im sozialgerichtlichen Verfahren für entsprechend anwendbar erklären, soweit VwGO und SGG keine eigenen Regelungen enthalten und die grundsätzlichen Unterschiede der Verfahrensarten dies nicht ausschließen. Überwiegend wird die entsprechende Anwendung der $\iint 5271$, 272, 275 ff. ZPO über die Vereinfachung und Beschleunigung des Verfahrens und damit auch des $\int 278$ Abs. 5 ZPO jedoch abgelehnt. ${ }^{123}$ Begründet wird dies teilweise unter Hinweis auf die grundsätzlichen Unterschiede von Zivilprozess auf der einen und verwaltungs- und sozialgerichtlichem Verfahren auf der anderen Seite, ${ }^{124}$ teilweise damit, dass $\int 87$ Abs. 1 S. 2 Nr. 1 VwGO eine eigenständige Regelung enthalte, die keinen Raum für eine weitere entsprechende Anwendung von \278 ZPO lasse. ${ }^{125}$

Die prozessuale Zulässigkeit eines richterlichen Hinweises auf die Möglichkeit der Inanspruchnahme eines außergerichtlichen oder gerichtlichen Mediationsangebotes ist im verwaltungs- und im sozialgerichtlichen Verfahren gleichwohl - auch ohne eine entsprechende Anwendung der ausdrücklichen Regelung des \278 Abs. 5 S. 2 und 3 ZPO - gegeben. ${ }^{126}$ Denn einem richterlichen Hinweis auf ein möglicherweise besser geeignetes Konfliktlösungsverfahren als das Gerichtsverfahren stehen die Regelungen von VwGO und SGG jedenfalls nicht entgegen. Gemäß S 173 VwGO in Verbindung mit \251 ZPO bzw. gemäß \202 SGG in Verbindung mit \251 ZPO kann zudem auch ohne Rückgriff auf \278 Abs. 5 S. 3 ZPO auf

\footnotetext{
122 Ausführliche Vorschläge hinsichtlich der verfahrenstechnischen Umsetzung der richterlichen Mediation finden sich bei Löer, ZZP 119 (2006), 199, 204 ff.

123 Dürschke, SGb 2001, 532, 533; Meyer-Ladewig, SGG, \ 202 Rn. 3; Ziekow, NVwZ 2004, 390, 394; Kopp/Schenke, VwGO, \173 Rn. 5; a.A. v. Bargen, DVBl 2004, 268, 275; differenzierend Meissner, in: Schoch/Schmidt-Aßmann/Pietzner, VwGO, Band 2, \173 Rn. 204, 206; für die Anwendbarkeit des $\int 279$ ZPO a.F. im Rahmen des verwaltungsgerichtlichen Verfahrens Alm-Merk, NdsVBl. 1997, 245, 248.

124 Dürschke, SGb 2001, 532, 533; Meyer-Ladewig, SGG, 』 202 Rn. 3.

125 Ziekow, NVwZ 2004, 390, 394; a.A. v. Bargen, DVB1 2004, 268, 275.

126 Ziekow, NVwZ 2004, 390, 396.
} 
Antrag der Konfliktparteien das Ruhen des Verfahrens angeordnet werden. ${ }^{127}$ Eine gesetzliche Regelung ist damit für die gerichtsnahe Mediation im verwaltungs- und im sozialgerichtlichen Verfahren nicht unbedingt erforderlich. ${ }^{128}$ Zur Förderung der einvernehmlichen Streitbeilegung und insbesondere der gerichtsnahen Mediation im Bereich des verwaltungs- und sozialgerichtlichen Verfahrens wäre es jedoch sinnvoll, eine dem \278 Abs. 5 S. 2 und 3 ZPO entsprechende Regelung in VwGO und SGG aufzunehmen ${ }^{129}$ bzw. die Regelung zumindest ausdrücklich für entsprechend anwendbar zu erklären. ${ }^{130}$

\section{Fazit}

58 Die vorstehenden Ausführungen zeigen, dass für die dauerhafte Einführung eines - rechtlich zulässigen - Mediationsangebotes noch erheblicher Handlungsbedarf besteht.

59 Die viel diskutierte Problematik der Zulässigkeit der Tätigkeit nicht-anwaltlicher Mediatoren wird im Bereich der gerichtsnahen Mediation zwar dadurch entschärft, dass Art. 1 \ 3 Nr. 1 RBerG Behörden die rechtliche Beratung im Rahmen ihrer Zuständigkeit erlaubt und das gerichtliche Mediationsangebot damit unter dem Aspekt der unerlaubten Rechtsberatung bereits nach geltendem Recht keinen Zulässigkeitsbedenken begegnet.

60 Dringend erforderlich ist es dagegen, für die richterliche Mediatorentätigkeit eine gesetzliche Grundlage im Sinne von $\int 4$ Abs. 2 Nr. 2 DRiG zu schaffen, um die dienstrechtliche Zulässigkeit zu gewährleisten.

61 Bei der dauerhaften Einführung von gerichtsnaher Mediation ist wegen wettbewerbsrechtlicher Bedenken eine rechtliche Anpassung dringend notwendig. Hier sind eine Änderung der ZPO und eine gesetzliche Verankerung der gerichtsnahen Mediation dringend zu empfehlen. Daneben wäre auch die Einführung einer angemessenen Kostenregelung für die gerichtsnahe Mediation möglich, um die Bedenken eines Verstoßes gegen das UWG durch ein dauerhaft kostenfreies gerichtliches Mediationsangebot auszuräumen.

\footnotetext{
127 Ziekow, NVwZ 2004, 390, 396; Meissner, in: Schoch/Schmidt-Aßmann/Pietzner, VwGO, Band 2, $\int 173 \mathrm{Rn} .206$.

128 Hält man \278 Abs. 5 S. 2 und 3 ZPO über $\int 173$ VwGO auch im verwaltungsgerichtlichen Verfahren für anwendbar, so stellt diese Regelung - wie im Zivilprozess im Übrigen auch - lediglich die ausdrückliche Legalisierung einer ohnehin schon zulässigen Praxis dar, siehe dazu auch Meissner, in: Schoch/Schmidt-Aßmann/Pietzner, VwGO, Band 2, 』173 Rn. 206.

${ }^{29}$ Ebenso v. Bargen, DVBl 2004, 268, 276; Ziekow, NVwZ 2004, 390, 396.

130 Pitschas, NVwZ 2004, 396, 402.
} 
Nicht zwingend geboten, zur Förderung der gerichtsnahen Mediation im Bereich des verwaltungs- und sozialgerichtlichen Verfahrens jedoch sinnvoll, wäre es zudem, eine dem $\int 278$ Abs. 5 S. 2 und 3 ZPO entsprechende Regelung in VwGO und SGG aufzunehmen bzw. \ 278 Abs. 5 S. 2 und 3 ZPO zumindest ausdrücklich für entsprechend anwendbar zu erklären.

\section{Die rechtlichen Beziehungen zwischen der Justiz und den an der gerichtsnahen Mediation beteiligten Parteien}

\section{Vertragsrechtliche Beziehungen}

Mangels gesetzlicher Regelung für das gerichtliche Mediationsangebot sind die rechtlichen Beziehungen zwischen den Parteien und dem Richtermediator bzw. der Justiz regelmäßig vertraglich geregelt. Vertragspartner der Konfliktparteien wird dabei nicht der Richtermediator, sondern - vergleichbar der Situation bei Abschluss eines Mediationsorganisationsvertrages mit einer Mediationsinstitution im außergerichtlichen Bereich ${ }^{131}$ - das jeweilige Bundesland als Rechtsträger der Justiz. ${ }^{132}$ Direkte vertragliche Beziehungen zwischen den Parteien und dem Richtermediator bestehen somit nicht, der Mediator ist vielmehr allein der Justiz als Mediationsinstitution verpflichtet. ${ }^{133} \mathrm{Im}$ Verhältnis zu den Konfliktparteien wird er für die Justiz als Erfüllungsgehilfe gemäß \ 278 BGB tätig. ${ }^{134}$ Die Parteien sind hinsichtlich der von der Justiz zu erbringenden Leistung Mitgläubiger gemäß \432

${ }^{131}$ Die Mediationsinstitution übernimmt in diesem Fall die Organisation des Mediationsverfahren, stellt die nötige Infrastruktur zur Verfügung und bestimmt unter Umständen auch den Mediator, siehe dazu Heß/Sharma, in: Haft/v. Schlieffen, Handbuch Mediation, S. 675, 686; Nölting, Mediatorenverträge, S. 72 .

132 Volkmann, Mediation im Zivilprozess. Rechtliche Rahmenbedingungen für ein gerichtsinternes Mediationsangebot, S. $94 \mathrm{ff}$, auch zum folgenden.

${ }^{133}$ Für den Bereich der außergerichtlichen Mediation Eidenmüller, Vertrags- und Verfahrensrecht der Wirtschaftsmediation, S. 33; Eidenmüller, in: Breidenbach/Coester-Waltjen/Heß/Nelle/C. Wolf, Konsensuale Streitbeilegung, S. 45, 71; Heß/Sharma, in: Haft/v. Schlieffen, Handbuch Mediation, S. 675, 686; für die vergleichbare Situation bei der institutionellen Schiedsgerichtsbarkeit Geimer, in: Zöller, ZPO, $\ 1035$ Rn. 23; Schlosser, in: Stein/Jonas, ZPO, Band 9, Vor $\int 1025$ Rn. 7; C. Wolf, Die institutionelle Handelsschiedsgerichtsbarkeit, S. 79 f. und 253; a.A. Nölting, Mediatorenverträge, S. 78.

134 Volkmann, Mediation im Zivilprozess. Rechtliche Rahmenbedingungen für ein gerichtsinternes Mediationsangebot, S. 95. 
BGB, ${ }^{135}$ im Hinblick auf eine - in der Zukunft geschuldete - Vergütung der Mediatorentätigkeit Gesamtschuldner im Sinne von \421 BGB. ${ }^{136}$

\section{a) Vertragsinhalt}

64 Hauptleistungspflicht des Mediators bzw. der Justiz, als die das Mediationsverfahren organisierende Institution, ist die sachkundige Unterstützung der Parteien bei dem Versuch einer einvernehmlichen Konfliktbeilegung. ${ }^{137}$ Welche Pflichten neben der Bereitstellung der für die Durchführung des Mediationsverfahrens erforderlichen Infrastruktur - davon im Einzelnen erfasst sein sollen, kann im Mediationsorganisationsvertrag geregelt werden. Teilweise werden zu diesem Zweck an den Projektgerichten schriftliche Vereinbarungen zwischen den Parteien und dem Richtermediator geschlossen, die auch die zwischen den Konfliktparteien zur Verabredung des Mediationsversuchs zu schließende Mediationsvereinbarung enthalten. Um eine einheitliche Praxis an den Gerichten zu gewährleisten, empfiehlt es sich jedoch zur Konkretisierung des Vertragsinhalts auf ein die Verhaltenspflichten von Mediatoren betreffendes Regelwerk zurückzugreifen. Dafür bietet sich insbesondere der von der EU-Kommission in Zusammenarbeit mit Vertretern verschiedener europäischer Konfliktbeilegungsinstitutionen entwickelte Verhaltenskodex für Mediatoren an: Darin sind neben dem Erfordernis einer ausreichenden fachlichen Qualifikation des Mediators insbesondere die Fragen der Unabhängigkeit, Neutralität und Unparteilichkeit des Mediators, der Verfahrensleitung, der Fairness des Verfahrens, der Verfahrensbeendigung, der Vergütung sowie der Verschwiegenheit des Mediators geregelt. ${ }^{138}$

65 Als Gegenleistung der Parteien wird, um die wettbewerbsrechtliche Zulässigkeit des gerichtlichen Mediationsangebotes zu gewährleisten, regelmäßig eine ange-

\footnotetext{
135 Für den Bereich der außergerichtlichen Mediation Eidenmüller, Vertrags- und Verfahrensrecht der Wirtschaftsmediation, S. 33; Eidenmüller, in: Breidenbach/Coester-Waltjen/Heß/Nelle/C. Wolf, Konsensuale Streitbeilegung, S. 45, 71 f.; Eidenmüller, in: Henssler/Koch, Mediation in der Anwaltspraxis, S. 39, 67; Hacke, Der ADR-Vertrag, S. 71.

${ }^{136}$ Für den Bereich der außergerichtlichen Mediation Eidenmüller, Vertrags- und Verfahrensrecht der Wirtschaftsmediation, S. 33; Eidenmüller, in: Breidenbach/Coester-Waltjen/Heß/Nelle/C. Wolf, Konsensuale Streitbeilegung, S. 45, 71 f.; Eidenmüller, in: Henssler/Koch, Mediation in der Anwaltspraxis, S. 39, 67; Hacke, Der ADR-Vertrag, S. 71; Heß/Sharma, in: Haft/v. Schlieffen, Handbuch Mediation, S. 675, 685.

${ }^{137}$ Siehe dazu für den außergerichtlichen Bereich Hacke, Der ADR-Vertrag, S. 67 ff.; Nölting, Mediatorenverträge, S. 19.

${ }^{138}$ European Code of Conduct for Mediators, abgedruckt in: ZKM 2004, 148 f.
} 
messene Vergütung der Mediationsdienstleistung in den Vertrag aufzunehmen sein. ${ }^{139}$

\section{b) Qualifizierung als öffentlich-rechtlicher Vertrag?}

\section{aa) Gerichtsnahe Mediation im Zivilprozess}

Fraglich ist, ob es sich bei dem zwischen der Justiz und den Parteien einer gerichtsnahen Mediation geschlossenen Mediationsorganisationsvertrag um einen privatrechtlichen oder einen öffentlich-rechtlichen Vertrag handelt. Gemäß \54 S. 1 VwVfG liegt ein öffentlich-rechtlicher Vertrag vor, wenn ein Rechtsverhältnis auf dem Gebiet des öffentlichen Rechts durch Vertrag begründet, geändert oder aufgehoben wird. Es kommt damit auf den Inhalt des Vertrags an, ${ }^{140}$ nicht dagegen darauf, ob auf einer Seite ein öffentlicher Rechtsträger beteiligt ist ${ }^{141}$ oder ob der Vertrag der Erfüllung öffentlicher Aufgaben dient. ${ }^{142}$

Vor diesem Hintergrund ist der zwischen Justiz und Parteien geschlossene Mediationsorganisationsvertrag als rein privatrechtlicher Vertrag zu qualifizieren. ${ }^{143}$ Denn die Justiz bietet ihre Mediationsdienstleistungen wie private Mediatoren auch - außerhalb des von ihr originär wahrzunehmenden Aufgabenbereichs - auf dem Mediationsmarkt an. Vertragsinhalt ist allein die Pflicht zur Unterstützung bei der Durchführung eines Mediationsversuchs. Ein Bezug zum öffentlichen Recht ist hier nicht gegeben.

\section{bb) Gerichtsnahe Mediation im verwaltungs- oder sozialgerichtli- chen Verfahren}

Allein auf der Grundlage des öffentlich-rechtlichen Charakters des Konfliktgegenstandes ist der Mediationsorganisationsvertrag auch im Bereich der öffentlichrechtlichen Gerichtsbarkeiten nicht als öffentlich-rechtlicher Vertrag im Sinne des

139 Volkmann, Mediation im Zivilprozess. Rechtliche Rahmenbedingungen für ein gerichtsinternes Mediationsangebot, S. 93 f.

140 BVerwGE 74, 368, 370; BVerwGE 30, 65, 67; BVerwGE 42, 331, 332; BVerwG NJW 1995, 1104, 1105; BGHZ 89, 250, 252; BGHZ 32, 215 f.; Kopp/Ramsauer, VwVfG, I 54 Rn. 27; Erichsen, in: Erichsen/Ehlers, Allgemeines Verwaltungsrecht, S. 405 f.; Maurer, Allgemeines Verwaltungsrecht, S. 368; Schlette, Die Verwaltung als Vertragspartner, S. $112 \mathrm{f}$.

141 So Gern, NJW 1979, 694, 695.

142 Bonk, in: Stelkens/Bonk/Sachs, VwVfG, I 54 Rn. 76; Schlette, Die Verwaltung als Vertragspartner, S. $119 \mathrm{f}$.

143 Volkmann, Mediation im Zivilprozess. Rechtliche Rahmenbedingungen für ein gerichtsinternes Mediationsangebot, S. 97. 
$\int 54 \mathrm{~S} .1 \mathrm{VwVfG}$ zu qualifizieren. Für vorgerichtlich durchgeführte Mediationsverfahren im Bereich des Verwaltungsrechts wurde der öffentlich-rechtliche Charakter des Mediatorvertrages mit einer Beleihung des Mediators oder mit der Konstruktion des Mediators als Verwaltungshelfer begründet. ${ }^{144}$ Nach anderer Ansicht wird der Mediator auf Grund eines privatrechtlichen Auftrags tätig, da er nicht für die Verwaltung, sondern gleichermaßen im Interesse aller Konfliktparteien tätig werde und er zudem in keinem Abhängigkeitsverhältnis zur Verwaltung stehe. ${ }^{145}$

69 Dies entspricht auch der Situation im Bereich der gerichtsnahen Mediation: Es handelt sich bei der gerichtsnahen Mediation um ein unabhängiges Angebot der Justiz, welches, solange eine öffentlich-rechtliche Regelung der gerichtlichen Mediationsangebote nicht existiert, ebenso wie die Tätigkeit privater Mediationsanbieter dem Privatrecht zuzuordnen ist. Dass sich das Mediationsverfahren auf Konflikte auf dem Gebiet des öffentlichen Rechts bezieht, ändert daran nichts.

\section{c) Vertragstypische Einordnung}

70 Nach Einführung der für das dauerhafte Angebot von gerichtsnaher Mediation erforderlichen Kostenregelung, wird der Mediationsorganisationsvertrag in den meisten Fällen als Dienstvertrag mit Geschäftsbesorgungscharakter gemäß \$S 611, 675 Abs. 1 BGB zu qualifizieren sein. ${ }^{146}$ Als Dienstvertrag ist die Vereinbarung deshalb anzusehen, weil sich der Mediator bzw. die Justiz weder zur Lösung des Konflikts ${ }^{147}$ noch zur Unterbreitung eines konkreten Vergleichsvorschlags verpflichtet ${ }^{148}$ und anders als bei einem Werkvertrag ein bestimmter Erfolg damit nicht geschuldet ist. Voraussetzung für das Vorliegen einer Geschäftsbesorgung im Sinne von $\int 675$ Abs. 1 BGB ist, dass es sich bei der Dienstleistung um eine

144 Hellriegel, Mediation im Umweltrecht, S. 190 ff.

145 Holznagel/Ramsauer, in: Haft/v. Schlieffen, Handbuch Mediation, S. 1124, 1131; Kopp/Ramsauer, VwVfG, Einf. Rn. 101.

146 Für den außergerichtlichen Bereich Eidenmüller, Vertrags- und Verfahrensrecht der Wirtschaftsmediation, S. 32; Eidenmüller, in: Breidenbach/Coester-Waltjen/Heß/Nelle/C. Wolf, Konsensuale Streitbeilegung, S. 45, 71; Eidenmüller, in: Henssler/Koch, Mediation in der Anwaltspraxis, S. 39, 67; Hacke, Der ADR-Vertrag, S. 77 ff.; C. Hartmann, in: Haft/v. Schlieffen, Handbuch Mediation, S. 712, 720; Heß/Sharma, in: Haft/v. Schlieffen, Handbuch Mediation, S.675, 685; Koch, in: Henssler/Koch, Mediation in der Anwaltspraxis, S. 245, 251; Leibner, NJW 2002, 3521; Monßen, AnwB1 2001, 169; Nölting, Mediatorenvertrage, S. 23; Prütting, in: Haft/v. Schlieffen, Handbuch Mediation, S. 824, 827; Wegmann, Zeitschrift für Mediation 1999, 146, 147; a.A. Glenewinkel, Mediation als außergerichtliches Konfliktlösungsmodell, S. 295 f.

147 Hacke, Der ADR-Vertrag, S. 75; Nölting, Mediatorenverträge, S. 23 f.; Prütting, in: Haft/v. Schlieffen, Handbuch Mediation, S. 824, 829.

148 Hacke, Der ADR-Vertrag, S. 74. 
selbständige Tätigkeit wirtschaftlicher Art handelt, die nicht in der bloßen Dienstoder Werkleistung gegenüber einem anderen, sondern in der selbständigen Wahrnehmung der Vermögensinteressen eines anderen besteht. ${ }^{149}$ Auch dies ist im Fall der gerichtsnahen Mediation regelmäßig der Fall: Der Richtermediator handelt (für die Justiz) selbständig, indem er das Mediationsverfahren leitet und regelmäBig unabhängig von Weisungen der Parteien eigenverantwortlich gestaltet. ${ }^{150}$ Die Fremdnützigkeit der Mediatorentätigkeit ergibt sich daraus, dass der Mediator durch die Unterstützung der Parteien bei dem Versuch einer Konfliktlösung in erster Linie das fremde Interesse der Parteien fördert. ${ }^{151}$ Indem die Gerichte mit ihrem Mediationsangebot in Konkurrenz zu privaten Mediationsanbietern treten, nehmen sie aktiv am Wirtschaftsleben teil, so dass es sich auch um eine Tätigkeit wirtschaftlicher Art handelt. ${ }^{152}$ Schließlich geht es bei der einvernehmlichen Beilegung von Rechtsstreitigkeiten in den meisten Fällen auch um Vermögensinteressen der Konfliktparteien, so dass in der Tätigkeit des Richtermediators ohne Weiteres eine Geschäftsbesorgung im Sinne von \ 675 Abs. 1 BGB zu sehen ist.

Wenn der Bezug zum Vermögen der Konfliktparteien dagegen fehlt, handelt es sich um einen reinen Dienstvertrag gemäß \611 BGB. Dies kann etwa in familienrechtlichen Streitigkeiten bei Verhandlungen über das Umgangs- und Sorgerecht für gemeinsame Kinder der Fall sein. ${ }^{153}$

\section{Haftungsfragen}

Da die Rechtsbeziehungen zwischen Justiz und Konfliktparteien bei der gerichtsnahen Mediation durch privatrechtlichen Vertrag geregelt sind, erfolgt eine Haftung der Justiz nicht nach den allgemeinen Amtshaftungsgrundsätzen gemäß S 839 BGB, sondern gemäß 278 BGB. Als mögliche Anspruchsgrundlagen kommen dabei insbesondere die $\iint 280$ ff. BGB in Betracht. Daneben kommt eine vertragliche Haftung gemäß \627 Abs. 2 BGB auch in Zusammenhang mit einer

149 Häuser/Welter, in: Soergel, BGB, Band 4/2, \ 675 Rn. 2; Sprau, in: Palandt, BGB, \675 Rn. 2 ff.; Martinek, in: Staudinger, BGB, JS 652-704, \675 Rn. A 23; Medicus, Schuldrecht, Rn. 433.

150 S. dazu Eidenmüller, Vertrags- und Verfahrensrecht der Wirtschaftsmediation, S. 34; Eidenmüller, in: Breidenbach/Coester-Waltjen/Heß/Nelle/C. Wolf, Konsensuale Streitbeilegung, S. 45, 72; Hager, Konflikt und Konsens, S. 114.

151 Dass dabei möglicherweise auch eigene Interessen verfolgt werden, etwa die Entlastung der Justiz, ist dabei unerheblich. Allgemein zur Fremdnützigkeit der Geschäftsbesorgung Beutbien, in: Soergel, BGB, Band 4/2, \662 Rn. 10; Sprau, in: Palandt, BGB, \ 662 Rn. 7; Wittmann, in: Stau-

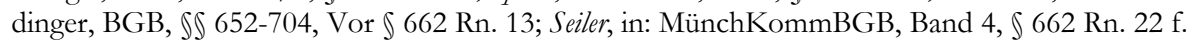

152 Zum Kriterium der „Tätigkeit wirtschaftlicher Art“ siehe Häuser/Welter, in: Soergel, BGB, Band 4/2, \675 Rn. 5; Martinek, in: Staudinger, BGB, JS 652-704, 』675 Rn. A 30.

153 Siehe dazu Nölting, Mediatorenverträge, S. 27. 
unzeitigen außerordentlichen Kündigung durch den Richtermediator in Betracht. Theoretisch möglich ist aber auch eine Haftung nach den Grundsätzen des Deliktsrechts etwa wegen Verletzung eines Schutzgesetzes gemäß \823 Abs. 2 BGB bei Missachtung der dem Richtermediator gemäß \203 Abs. 2 S. 1 StGB obliegenden Schweigepflicht.

73 Anspruchsschuldner ist bei einem haftungsbegründenden Verhalten des Richtermediators in erster Linie die Justiz, die gemäß \278 BGB für das Verschulden ihres Erfüllungsgehilfen haftet. Nur im Falle deliktischen Handelns kann daneben auch der Richtermediator selbst in Anspruch genommen werden, wobei er als Beamter im staatsrechtlichen Sinne ${ }^{154}$ allerdings nur nach 839 BGB haftet. Das bedeutet, dass die persönliche Haftung des Richtermediators gemäß \839 Abs. 1 S. 2 BGB jedenfalls dann nicht in Betracht kommt, wenn die Konfliktparteien bereits die Justiz gemäß \831 BGB in Anspruch nehmen können und der Richter nicht vorsätzlich gehandelt hat. Ob die für eine Haftung der Justiz gemäß \ 831 BGB erforderliche Weisungsgebundenheit des Richters gegeben ist, hängt davon $\mathrm{ab}$, wo die richterliche Mediatorentätigkeit in Zukunft angesiedelt wird. ${ }^{155}$ Vor dem Hintergrund, dass die richterliche Mediatorentätigkeit im Rahmen des niedersächsischen Modellprojektes als Aufgabe der Gerichtsverwaltung begriffen wird, wird in Niedersachsen derzeit wohl von einer Weisungsgebundenheit des Richtermediators ausgegangen.

74 Eine Haftungsbegrenzung in direkter oder analoger Anwendung des Spruchrichterprivilegs gemäß $\ 839$ Abs. 2 S. 1 BGB auf die richterliche Mediatorentätigkeit scheidet aus. ${ }^{156}$ Nach dieser Vorschrift ist ein Beamter, der bei einem Urteil in einer Rechtssache seine Amtspflicht verletzt, für den daraus entstehenden Schaden nur dann verantwortlich, wenn die Verletzung in einer Straftat besteht. Da der Anwendungsbereich des $₫ 839$ Abs. 2 S. 1 BGB damit jedenfalls auf richterliche Entscheidungen begrenzt ist, scheidet eine direkte Anwendbarkeit der Vorschrift von vornherein aus und es kommt wegen der fehlenden Vergleichbarkeit auch eine analoge Anwendung des Spruchrichterprivilegs auf die Tätigkeit des Richter-

\footnotetext{
${ }^{154}$ Für die Eigenhaftung des Amtsträgers in Ausübung privatrechtlicher Funktionen des Dienstherrn liegt $\int 839$ BGB der staatsrechtliche Beamtenbegriff zugrunde. Beamter im staatsrechtlichen Sinne ist, wer in einem öffentlich-rechtlichen Dienst- und Treueverhältnis zum Staat steht, in das er unter Aushändigung der gesetzlich vorgeschriebenen Ernennungsurkunde berufen worden ist, siehe

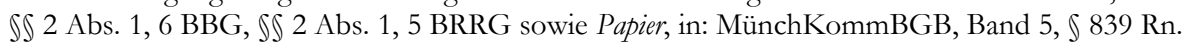
130.

155 Volkmann, Mediation im Zivilprozess. Rechtliche Rahmenbedingungen für ein gerichtsinternes Mediationsangebot, S. 107.

156 So auch Prütting, ZKM 2006, 100, 101.
} 
mediators nicht in Betracht. ${ }^{157}$ Ebensowenig ist von einem stillschweigend vereinbarten Haftungsausschluss entsprechend \839 Abs. 2 S. 1 BGB auszugehen. Denn anders als bei der Tätigkeit des Schiedsrichters, für den wegen der grundsätzlichen Vergleichbarkeit seiner Tätigkeit mit der eines staatlichen Richters eine solche stillschweigende Haftungsbegrenzung angenommen wird, ${ }^{158}$ kann bei der Tätigkeit eines (Richter-)Mediators, der keinesfalls eine der Rechtskraft fähige Entscheidung trifft, eine solche Vergleichbarkeit nicht festgestellt werden. ${ }^{159}$ Ausdrücklich vereinbarte Haftungsbegrenzungen bleiben im Rahmen der vom Gesetz gezogenen Grenzen dagegen zulässig.

\section{Fazit}

Die rechtlichen Beziehungen zwischen den Parteien einer Mediation und der Justiz können umfassend mit den Mitteln des Vertragsrechts durch privatrechtliche Vereinbarungen geregelt werden. Im Hinblick auf die Regelung der Pflichten des Mediators und der von ihm bei der Leitung des Mediationsverfahrens zu beachtenden Grundsätze empfiehlt sich dabei die Einbeziehung eines Regelwerks wie etwa des europäischen Verhaltenskodexes für Mediatoren, um weitgehend einheitliche Grundlagen für das gerichtliche Mediationsangebot an den einzelnen Gerichten sicherzustellen.

In Fällen, in denen es zu einer Haftung im Mediationsverfahren aufgrund eines Fehlers des Mediators kommt, werden grundsätzlich nur Ansprüche gegen die Justiz bestehen, die gemäß \ 278 BGB für das Verschulden ihres Erfüllungsgehilfen haftet. Als Anspruchsgrundlage dienen dabei regelmäßig die \$S 280 ff. BGB. Lediglich in den Fällen, in denen der Mediator deliktisch handelt, kommt ausnahmsweise eine Amtshaftung nach $\int 839$ BGB in Betracht. Dabei scheidet jedoch eine Haftungsbegrenzung, wie sie in $\int 839$ Abs. 2 S. 1 für den Spruchrichter besteht, aus, da dessen Anwendungsbereich auf richterliche Entscheidungen begrenzt ist.

\footnotetext{
157 So auch für den Bereich der außergerichtlichen Mediation unter Verweis auf die funktionalen Unterschiede von Rechtsprechung und Mediation Eidenmüller, Vertrags- und Verfahrensrecht der Wirtschaftsmediation, S. 41; Eidenmüller, in: Breidenbach/Coester-Waltjen/Heß/Nelle/C. Wolf, Konsensuale Streitbeilegung, S. 45, 78; Brieske, in: Henssler/Koch, Mediation in der Anwaltspraxis, S. 271, 273 f.; Heß/Sharma, in: Haft/v. Schlieffen, Handbuch Mediation, S. 675, 688; Nölting, Mediatorenverträge, S. 202 ff.; Nölting, ZKM 2004, 231, 234.

158 BGHZ 15, 12, 15; BGHZ 42, 313, 316.

${ }^{159}$ Für die außergerichtliche Mediation Hacke, Der ADR-Vertrag, S. 342; Brieske, in: Henssler/Koch, Mediation in der Anwaltspraxis, S. 271, 273 f.; Eidenmüller, Vertrags- und Verfahrensrecht der Wirtschaftsmediation, S. 41; Eidenmüller, in: Breidenbach/Coester-Waltjen/Heß/Nelle/C. Wolf, Konsensuale Streitbeilegung, S. 45, 78.
} 


\section{Die rechtliche Beziehung zwischen den an der gerichtsnahen Mediation beteiligten Konfliktparteien}

77 Die rechtliche Beziehung zwischen den an der gerichtsnahen Mediation beteiligten Konfliktparteien soll nachfolgend insbesondere unter den Aspekten der Durchsetzbarkeit, der Gefahr von Rechtsverlusten während des Mediationsverfahrens und der Vertraulichkeit näher betrachtet werden. In diesen Punkten dürfen sich gegenüber dem streitigen Verfahren keine Nachteile für die Parteien ergeben, wenn keine negativen Anreize für die Wahl der gerichtsnahen Mediation gesetzt werden sollen. Die Klärung dieser rechtlichen Rahmenbedingungen ist daher notwendige Voraussetzung für die spätere Analyse über Kosten- und Anreizstrukturen in der Mediation:

\section{Durchsetzbarkeit}

\section{a) Materiell-rechtlicher Vergleich}

\section{aa) Vergleichsschluss bei Mediation im Zivilprozess}

78 Bei einer in der Mediation von den Parteien getroffenen Vereinbarung wird es sich in der Regel um einen materiell-rechtlichen Vergleich im Sinne von \779 S. 1 BGB und damit um einen rechtlich bindenden Vertrag handeln. Die Erfüllung dieser Vereinbarung wäre allerdings nur dann gewährleistet, wenn ein Bruch der Vereinbarung ineffizient bzw. ausgeschlossen wäre. ${ }^{160} \mathrm{Zu}$ denken ist in diesem Zusammenhang zunächst an die „Drohung“ mit gerichtlicher Durchsetzung der Vereinbarung. Mediationsverhandlungen werden insoweit häufig als „bargaining in the shadow of the law" "161 angesehen, als einvernehmliche Vereinbarungen erzielt werden, deren Einhaltung vertraglich festgehalten und damit einer Durchsetzung mit Hilfe der staatlichen Gerichte zugänglich gemacht werden. ${ }^{162}$ Der Erwartungswert der Erzwingung der Vertragserfüllung durch ein Gericht ist es, der für die Stabilität der Übereinkunft sorgt. ${ }^{163}$ Mit Blick auf Prozessrisiko und Zeitaufwand einer etwaigen Erfüllungsklage entspricht es jedoch dem Interesse der Par-

\footnotetext{
160 Weigel, in: FS Ott, S. 491, 492 f.

161 Mnookin/Kornhauser, 88 Yale Law Journal 950 ff. (1979).

162 Weigel, in: FS Ott, S. 491, 499.

163 Weigel, in: FS Ott, S. 491, 493.
} 
teien, darüber hinaus Maßnahmen zur Sicherung der Erfüllung ihres Vertrages zu ergreifen. ${ }^{164}$

In Betracht kommt die Einbeziehung materiell-rechtlicher Sanktionen in den in der Mediation geschlossenen Vergleich. So kann aus dem Mediationsvergleich beispielsweise durch die bilaterale Stellung liquider Sicherheiten ein sich selbst durchsetzender Vertrag (self-enforcing agreement) gemacht werden. ${ }^{165}$ Allerdings kann selbst eine solche Vertragsgestaltung keine absolute Garantie für die Erfüllung des Vertrages bieten, so dass darüber hinaus ein Bedürfnis bestehen kann, über die in der Mediation getroffene Vereinbarung einen Vollstreckungstitel zu erhalten und gegebenenfalls die Vereinbarung im Wege der Zwangsvollstreckung durchzusetzen.

\section{bb) Zulässigkeits- und Wirksamkeitsgrenzen für den Vergleichs- schluss bei Mediation im verwaltungs- oder sozialgerichtlichen Verfahren}

Soweit im Rahmen der Mediation zur Lösung eines Konfliktes auf dem Gebiet des öffentlichen Rechts zwischen den Konfliktparteien ein Vergleich geschlossen wird, ist $\mathrm{zu}$ beachten, dass es sich dann in aller Regel um einen öffentlichrechtlichen Vertrag gemäß \54 S. 1 VwVfG bzw. \53 Abs. 1 SGB X X ${ }^{166}$ handelt. Denn Gegenstand des Mediationsvergleichs ist in diesen Fällen ein „Rechtsverhältnis auf dem Gebiet des öffentlichen Rechts“ im Sinne von \54 S. 1 VwVfG bzw. \53 SGB X. Etwas anderes gilt nur dann, wenn im Rahmen eines Mediationsverfahrens mit mehreren Konfliktparteien der Vergleich ohne Beteiligung der Verwaltung allein zwischen Privaten geschlossen wird. Zwar sind grundsätzlich auch öffentlich-rechtliche Verträge zwischen Privaten möglich, in den meisten Fällen wird aber davon auszugehen sein, dass - trotz des Bezugs des gesamten Konflikts zum öffentlichen Recht - durch einen allein zwischen Privaten geschlossenen Vertrag auch nur privatrechtliche Pflichten und Rechte begründet werden sollen.

164 Eidenmüller, in: Breidenbach/Coester-Waltjen/Heß/Nelle/Wolf, Konsensuale Streitbeilegung, S. 45,80 .

165 Eidenmüller, in: Breidenbach/Coester-Waltjen/Heß/Nelle/Wolf, Konsensuale Streitbeilegung, S. 45, 80; ähnlich auch Deason, Public Law and Legal Theory Working Paper Series No. 22/Center for Law, Policy and Social Science Working Paper Series No. 15, S. 38.

166 Die entsprechenden Normen des SGB X sind meist wörtlich denen des VwVfG nachgebildet. Soweit im SGB X Abweichungen gegenüber dem VwVfG bestehen, werden diese kenntlich gemacht. 
81 Als Besonderheit für das sozialgerichtliche Verfahren ist zu beachten, dass gem. \ 53 Abs. 2 SGB X ein öffentlich-rechtlicher Vertrag über Sozialleistungen nur geschlossen werden kann, soweit die Erbringung der Leistung im Ermessen des Leistungsträgers steht. Auf Sozialleistungen (nach \ 11 SGB Dienst-, Sach- und Geldleistungen) besteht gem. \38 SGB I grundsätzlich ein Anspruch, soweit dem Leistungsträger nicht ausdrücklich ein Ermessen eingeräumt ist. Daher unterliegen weite Bereiche der Sozialleistungen nicht der Regelung durch einen öffentlichrechtlichen Vertrag.

82 Beim Abschluss eines öffentlich-rechtlichen Vertrages sind die folgenden Zulässigkeits- und Wirksamkeitsgrenzen zu beachten:

\section{(1) Verfassungsrechtliche Vorgaben des Gebots von Vorrang und Vorbehalt des Gesetzes}

83 Allgemein ist bei Abschluss eines öffentlich-rechtlichen Vertrages die Bindung der Verwaltung an die Grundsätze des Vorrangs und des Vorbehalts des Gesetzes zu beachten. Durch den Grundsatz des Vorrangs des Gesetzes wird die Dispositionsbefugnis der Verwaltung im Rahmen von Mediationsverhandlugen insoweit eingeschränkt, als sie sich nicht zu rechtswidrigen Leistungen verpflichten darf. ${ }^{167}$ Im Hinblick auf den Grundsatz des Vorbehalts des Gesetzes gilt zwar, dass es nicht erforderlich ist, dass jede der in dem Vertrag getroffenen Vereinbarungen durch eine über $\int 54 \mathrm{VwVfG}$ hinausgehende spezielle Ermächtigungsgrundlage gedeckt ist. ${ }^{168}$ Doch erlaubt $\int 54$ S. 1 VwVfG weder vertragliche Regelungen, die gegen den Grundsatz des Vorrangs des Gesetzes verstoßen, noch die Verpflichtung zu oder die Gewährung von Leistungen, die dem Vorbehalt des Gesetzes unterliegen, entgegen diesem Grundsatz. ${ }^{169}$

\section{(2) Gleichbehandlungsgrundsatz}

84 Grenzen für ein zulässiges Verhandlungsergebnis im Rahmen der gerichtsnahen Mediation an den Verwaltungs- und Sozialgerichten werden auch durch den Gleichbehandlungsgrundsatz gemäß Art. 3 Abs. 1 GG gesetzt. ${ }^{170}$ Insbesondere im Bereich der Leistungsgewährung durch die Verwaltung ist zu beachten, dass Art. 3 Abs. 1 GG eine Selbstbindung der Verwaltung bei der Ausübung von Ermessen

\footnotetext{
167 Alm-Merk, NdsVBl. 1997, 245, 247.

168 Kopp/Ramsauer, VwVfG, $\int 54$ Rn. 44; Ule/Laubinger, Verwaltungsverfahrensgesetz, S. 784.

${ }^{169}$ Kopp/Ramsauer, VwVfG, \54 Rn. 44; Bonk, in: Stelkens/Bonk/Sachs, VwVfG, \54 Rn. 13.

170 Alm-Merk, NdsVBl. 1997, 245, 247, 249; Engelmann, in von Wulffen, SGB X, \53 Rn. 15.
} 
fordert, was den Entscheidungsspielraum der Verwaltung bei der Erarbeitung einer für beide Seiten akzeptablen Konfliktlösung erheblich einschränkt. ${ }^{171}$

\section{(3) Vertragsformverbote}

Aus dem in $\int 54$ S. 1 VwVfG bzw. \53 Abs. 1 S. 1 SGB X enthaltenen Vorbehalt „soweit Rechtsvorschriften nicht entgegen stehen“ ergibt sich zudem ein Verbot zum Abschluss eines öffentlich-rechtlichen Vertrages unter Verstoß gegen ein Vertragsformverbot. ${ }^{172}$ Ein Vertragsformverbot besteht, wenn eine Rechtsvorschrift ausdrücklich oder implizit anordnet, dass sich die Behörde einer anderen Handlungsform, etwa eines Verwaltungsaktes bedienen muss. ${ }^{173}$ Ein gleichwohl unter Verstoß gegen ein solches Handlungsformverbot geschlossener öffentlichrechtlicher Vertrag ist nichtig. ${ }^{174}$

\section{(4) Zustimmung nicht-beteiligter Dritter gemäß $\int 58$ Abs. 1 VwVfG}

Gemäß 58 Abs. 1 VwVfG bzw. \57 SGB X wird ein öffentlich-rechtlicher Vertrag, der in Rechte Dritter eingreift, erst wirksam, wenn der Dritte schriftlich zustimmt. Liegt ein Eingriff in Rechte Dritter vor, so ist die Vereinbarung so lange schwebend unwirksam, bis der betroffene Dritte die Zustimmung erteilt oder endgültig verweigert hat oder die Zustimmung als endgültig verweigert anzusehen ist. 175 Voraussetzung für einen Eingriff im Sinne des $\int 58$ Abs. 1 VwVfG bzw. \ 57 SGB X ist, dass ein subjektiv öffentliches Recht des Dritten in rechtlich relevanter Weise beeinträchtigt wird. ${ }^{176}$ Dies ist unzweifelhaft der Fall, wenn die vertragliche Regelung unmittelbar zu einer Rechtsbeeinträchtigung des Dritten führt, etwa bei Verträgen, durch die eine Genehmigung in Form eines Verwaltungsaktes

171 Spellbrink, SGb 2003, 141, 143; siehe auch Bonk, in: Stelkens/Bonk/Sachs, VwVfG, \ 54 Rn. 13; Knack, VwVfG, \54 Rn. 26.

172 Ule/Laubinger, Verwaltungsverfahrensrecht, S. 781; Bonk, in: Stelkens/Bonk/Sachs, VwVfG, \54 Rn. 13; Knack, VwVfG, \54 Rn. 25.

173 Ule/Laubinger, Verwaltungsverfahrensrecht, S. 781; Knack, VwVfG, \54 Rn. 18. In der verwaltungsrechtlichen Praxis bilden Vertragsformverbote jedoch die Ausnahme, siehe dazu $U$ -

le/Laubinger, Verwaltungsverfahrensrecht, S. 781; Bonk, in: Stelkens/Bonk/Sachs, VwVfG, \54 Rn. 103.

174 Ule/Laubinger, Verwaltungsverfahrensrecht, S. 782; Bonk, in: Stelkens/Bonk/Sachs, VwVfG, \54 Rn. 102; Knack, VwVfG, \54 Rn. 19.

175 Kopp/Ramsauer, VwVfG, \58 Rn. 9; Bonk, in: Stelkens/Bonk/Sachs, VwVfG, \58 Rn. 4; Knack, VwVfG, \ 58 Rn. 13; Engelmann, in: von Wulffen, SGB X, \ 57 Rn. 7, Diering in LPK-SGB X \57, Rn.9.

176 Kopp/Ramsauer, VwVfG, \ 58 Rn. 5; siehe auch Bonk, in: Stelkens/Bonk/Sachs, VwVfG, \ 58 Rn. 13 f.; Engelmann, in: von Wulffen, SGB X, \ 57 Rn. 4; Diering in LPK-SGB X \ 57, Rn. 4. 
(mit Doppelwirkung) ersetzt wird. ${ }^{177}$ Fraglich ist indes, ob $\int 58$ Abs. 1 VwVfG bzw. \57 SGB X auch auf Verpflichtungsverträge anwendbar ist, wenn sich die Behörde vertraglich zu einer Maßnahme oder einem Verhalten verpflichtet hat, welches zu einem Eingriff in die Rechte Dritter führt. Denn in der bloßen Verpflichtung der Behörde liegt noch keine Beeinträchtigung, so dass vom Wortlaut her die bloße Verpflichtung zum Erlass eines drittbelastenden Verwaltungsaktes nicht in den Anwendungsbereich des $\int 58$ Abs. 1 VwVfG bzw. $\int 57$ SGB X fällt. ${ }^{178}$ Wenn jedoch die Durchführung des Vertrages ohne eine Beeinträchtigung der Rechte Dritter nicht möglich ist, so ist mit der herrschenden Meinung davon auszugehen, dass vom Sinn und Zweck der Regelung her das Zustimmungserfordernis auch für Verpflichtungsverträge der Behörde gilt. ${ }^{179}$ Die generelle Verneinung einer Eingriffswirkung durch den Abschluss eines drittbelastenden Verpflichtungsvertrages hätte sonst de facto die Anerkennung öffentlich-rechtlicher Verträge zu Lasten Dritter zur Folge. ${ }^{180}$ Von der rechtsbeeinträchtigenden Wirkung eines Verpflichtungsvertrages ist daher auszugehen, wenn die Erfüllung des Vertrages Rechte Dritter verletzen kann. ${ }^{181}$

\section{(5) Form- und Zuständigkeitsfragen}

87 Die Rechtmäßigkeit des Mediationsvergleichs hängt ferner davon ab, dass die Behörde für den Abschluss des Vergleichs sachlich und örtlich zuständig ist und die für den Abschluss eines öffentlich-rechtlichen Vertrages vorgeschriebene Form, d.h. Schriftform gemäß $\ 57$ VwVfG bzw. \56 SGB X eingehalten wird.

\section{(6) Zwischenergebnis}

88 Festzuhalten bleibt für die gerichtlichen Mediationsangebote in den öffentlichrechtlichen Gerichtsbarkeiten, dass die Möglichkeiten eine rechtlich verbindliche Vereinbarung zu schließen gegenüber den Möglichkeiten für einen Vergleichsschluss im Zivilrecht deutlich eingeschränkt sind.

\footnotetext{
177 Kopp/Ramsauer, VwVfG, \58 Rn. 7; siehe dazu auch Bonk, in: Stelkens/Bonk/Sachs, VwVfG, \ 58 Rn. 14 sowie die Beispiele bei Knack, VwVfG, \ 58 Rn. 4.

178 Ule/Laubinger, Verwaltungsverfahrensrecht, \ 26 Rn. 8.

179 BVerwG NJW 1988, 663; OVG Münster, NVwZ 1988, 370; Bonk, in: Stelkens/Bonk/Sachs, VwVfG, \ 58 Rn. 15; Knack, VwVfG, \ 58 Rn. 6; Kopp/Ramsauer, VwVfG, \ 58 Rn. 7.

180 Kopp/Ramsauer, VwVfG, \ 58 Rn. 7; Bonk, in: Stelkens/Bonk/Sachs, VwVfG, \58 Rn. 16; Knack, VwVfG, \ 58 Rn. 6.; Engelmann, in: von Wulffen, SGB X, \57 Rn. 5.

181 Kopp/Ramsauer, VwVfG, \ 58 Rn. 8. Siehe dazu auch Knack, VwVfG, \ 58 Rn. 6.
} 


\section{b) Vollstreckungstitel}

\section{aa) Zivilprozess}

\section{(1) Prozessvergleich}

Im Bereich der gerichtsnahen Mediation haben die Parteien regelmäßig die Möglichkeit, die in der Mediation über die streitgegenständlichen Forderungen getroffenen Vereinbarungen anschließend als gerichtlichen Vergleich protokollieren zu lassen. Voraussetzung für einen Prozessvergleich ist, dass die Parteien den Prozess zumindest bezüglich eines Teils des Streitgegenstandes beenden wollen. ${ }^{182}$ Neben der zwingend erforderlichen Vereinbarung über die Erledigung zumindest eines Teils des Streitgegenstandes kann der Prozessvergleich darüber hinaus auch Angelegenheiten regeln, die außerhalb des Prozesses streitig sind oder Dritte betreffen. ${ }^{183}$ Die Parteien haben damit die Möglichkeit, auch Angelegenheiten, die Gegenstand der Mediationsverhandlungen, nicht jedoch des anhängigen gerichtlichen Verfahrens sind, in ihre Vereinbarung mit einzubeziehen und als Prozessvergleich protokollieren zu lassen.

Von der Konzeption des Modellversuchs her erfolgt die Protokollierung des Prozessvergleichs in der Weise, dass das gerichtliche Verfahren durch den gesetzlich zuständigen Richter wieder aufgenommen wird und dieser den in der Mediation geschlossenen Vergleich zu Protokoll nimmt. In der Praxis hat sich diese Vorgehensweise jedoch als umständlich herausgestellt, da für die Protokollierung des Prozessvergleichs vielfach ein zusätzlicher, für die Parteien bzw. ihre Anwälte zeitaufwändiger Termin anberaumt werden musste. ${ }^{184}$ Teilweise wurde daher an den Projektgerichten dazu übergegangen, den Prozessvergleich direkt im Anschluss an das Mediationsverfahren durch den Richtermediator zu Protokoll nehmen zu lassen. ${ }^{185}$ Verfahrensrechtlich soll diese Vorgehensweise dadurch abgesichert werden, dass der gesetzlich zuständige Richter vor Beginn der Mediation einen Beschluss erlässt, in dem ,der mit der Mediation betraute Richter [...] als ersuchter Richter gemäß $\iint 278$ Abs. 5 S. 1, 362 ZPO analog ersucht [wird], auf Wunsch der Parteien nach Beendigung der Mediation das Verfahren wieder aufzu-

\footnotetext{
182 Wolfsteiner, in: MünchKommZPO, Band 2, \ 794 Rn. 52; Stöber, in: Zöller, ZPO, \ 794 Rn. 2.

183 Münz̧berg, in: Stein/Jonas, ZPO, Band 6, \ 794 Rn. 11; Stöber, in: Zöller, ZPO, \ 794 Rn. 3; Wolfsteiner, in: MünchKommZPO, Band 2, \ 794 Rn. 57.

${ }^{184}$ Löer, ZZP 119 (2006), 119, 209.

185 So zuerst die Praxis am Landgericht Göttingen, siehe Götz v. Olenhusen, ZKM 2004, 104, 105.
} 
rufen und eine Güteverhandlung - einschließlich der Protokollierung eines ggf. abzuschließenden Vergleiches - durchzuführen." ${ }^{\text {"186 }}$

91 Zulässigkeitsbedenken dagegen, dass der mit der Mediation betraute Richter aus Praktikabilitätsgründen für den an sich zuständigen Richter analog $\iint 278$ Abs. 5 S. 1, 362 ZPO die Wiederaufnahme des Verfahrens und die anschließende Protokollierung des Prozessvergleichs übernimmt, bestehen nicht. Eine Analogie ist die Übertragung einer für einen Tatbestand (A) im Gesetz gegebenen Regel auf einen vom Gesetz nicht geregelten, vergleichbaren Tatbestand (B). ${ }^{187}$ Eine Analogie setzt damit das Bestehen einer Gesetzeslücke, d.h. eine planwidrige Unvollständigkeit des positiven Rechts voraus. ${ }^{188}$ Planwidrig ist eine solche Lücke, wenn im positiven Recht etwas nicht geregelt ist, obwohl die Rechtsordnung eine entsprechende Regelung fordert. ${ }^{189}$ Für die Feststellung einer planwidrigen Regelungslücke sind dabei insbesondere die Wertungen des Gesetzes als ein wesentlicher Bestandteil des geltenden Rechts in Verbindung mit dem Gleichheitssatz heranzuziehen. ${ }^{190}$

92 Für die hier in Frage stehende Situation der Protokollierung des Prozessvergleichs durch den Richtermediator ist von einer solchen planwidrigen Unvollständigkeit der Bestimmungen der ZPO auszugehen. Eine Protokollierung des Prozessvergleichs durch den Richtermediator direkt im Anschluss an die Mediationsverhandlungen ist gesetzlich nicht geregelt. Doch lässt sich vor dem Hintergrund der grundsätzlichen Vergleichbarkeit dieser Situation mit dem Fall einer Protokollierung des Prozessvergleichs durch einen ersuchten Richter in der Güteverhandlung eine Analogie mit der Wertung der \$S 278 Abs. 5 S. 1, 362 ZPO begründen. ${ }^{191}$ \ 362 ZPO regelt - etwa bei großer örtlicher Entfernung - die Beweisaufnahme durch ein anderes Gericht, das im Wege der Rechtshilfe (\$157 Abs. 1 GVG) tätig wird. Mit der Einführung des $\ 278$ Abs. 5 S. 1 ZPO, der für die Möglichkeit einer Güteverhandlung vor einem ersuchten Richter auf $\int 362$ ZPO verweist, ist nunmehr auch für die Durchführung der obligatorischen Güteverhandlung einschließ-

\footnotetext{
186 Götzv. Olenhusen, ZKM 2004, 104, 105; Löer, ZZP 119 (2006), 119, 209.

187 Larenz/Canaris, Methodenlehre der Rechtswissenschaft, S. 202.

188 Canaris, Die Feststellung von Lücken im Gesetz, S. 25, 30.

189 Canaris, Die Feststellung von Lücken im Gesetz, S. 55; siehe dazu auch Larenz/Canaris, Methodenlehre der Rechtswissenschaft, S. 195.

190 Canaris, Die Feststellung von Lücken im Gesetz, S. 56 f.; Larenz/Canaris, Methodenlehre der Rechtswissenschaft, S. 195; Pawlowski, Einführung in die juristische Methodenlehre, Rn. 204, $214 \mathrm{a}$.

191 Volkmann, Mediation im Zivilprozess. Rechtliche Rahmenbedingungen für ein gerichtsinternes Mediationsangebot, S. 80 .
} 
lich der Protokollierung eines Prozessvergleichs ${ }^{192}$ die Möglichkeit eines Rechtshilfegesuchs ausdrücklich geregelt. Gegenstand eines Rechtshilfegesuchs ist dabei immer eine richterliche Amtshandlung, die das ersuchende Gericht kraft eigener Zuständigkeit rechtlich auch selbst vornehmen könnte, zu deren Vornahme es sich aber aus tatsächlichen Gründen nicht oder jedenfalls nicht ohne Schwierigkeiten in der Lage sieht und die es deshalb aus Zweckmäßigkeitsgründen einem anderen Gericht übertragen hat. ${ }^{193}$

Vor diesem Hintergrund wird mit der entsprechenden Anwendung des $₫ 278$ Abs. 5 S. 1 ZPO auf den mit der Mediation betrauten Richter nur auf unbedenkliche Weise eine für die gerichtsnahe Mediation bestehende Regelungslücke geschlossen. Denn wenn schon Gründe der Praktikabilität es rechtfertigen, gemäß \\278 Abs. 5 S. 1, 362 ZPO einen Richter eines anderen Gerichts zu ersuchen, eine Güteverhandlung durchzuführen und unter Umständen auch einen Prozessvergleich zu Protokoll zu nehmen, so sollte dies für den Fall einer Protokollierung des Prozessvergleichs durch den Richtermediator im Anschluss an das Mediationsverfahrens erst recht gelten. ${ }^{194}$ In beiden Fällen besteht wegen der Aussicht auf einen ansonsten nicht unerheblichen Mehraufwand an Zeit und Kosten für die Beteiligten ein Bedürfnis dafür, dass für den an sich zuständigen gesetzlichen Richter ein anderer Richter, der für die Beteiligen - örtlich oder zeitlich - leichter zu erreichen ist, die Amtshandlung vornimmt. Ein sachlicher Grund für die ungleiche Behandlung beider Sachverhalte ist nicht ersichtlich. Vielmehr ist davon auszugehen, dass der Gesetzgeber bislang nur noch keine Gelegenheit hatte, die Bestimmungen der ZPO im Hinblick auf die neue Erscheinungsform eines gerichtsinternen Mediationsangebotes zu ergänzen.

Zur Klarstellung und um diesbezügliche Zweifel ${ }^{195}$ auszuräumen, empfiehlt sich gleichwohl eine Ergänzung des $\ 278$ Abs. 5 ZPO mit dem Inhalt, dass ein Richter, der im Rahmen eines gerichtlichen Mediationsangebotes die Mediationsverhandlungen leitet, durch den gesetzlich zuständigen Richter ersucht werden kann, das gerichtliche Verfahren nach Abschluss der Mediation wieder aufzurufen und einen von den Parteien in der Mediation geschlossenen Vergleich zu Proto-

\footnotetext{
192 Zweck der Güteverhandlung ist insbesondere auch die Prozessbeendigung durch Vergleich, siehe Greger, in: Zöller, ZPO, \$ 278 Rn. 9.

193 BGH NJW 1990, 2936.

194 v. Bargen, DVBl 2004, 468, 475, der allerdings $₫ 278$ Abs. 5 S. 1 ZPO auch auf die Tätigkeit des Richtermediator während des Mediationsverfahrens anwendet; Löer, ZKM 2005, 182, 185 f.;

Volkmann, Mediation im Zivilprozess. Rechtliche Rahmenbedingungen für ein gerichtsinternes Mediationsangebot, S. 81.

195 S. dazu Bischof, SchiedsVZ 2004, 252, 254.
} 
koll zu nehmen. Zudem wäre auch den Anforderungen des Art. 5 der Mediationsrichtlinie ${ }^{196}$ entsprochen, welcher - originär mit der Intention der Ermöglichung einer grenzüberschreitenden Vollstreckung ${ }^{197}$ die Schaffung einer Vollstreckbarerklärung bzw. -bestätigung des Mediationsergebnisses vorsieht.

\section{(2) Andere Vollstreckungstitel}

95 Daneben können die Parteien das anhängige Gerichtsverfahren jedoch auch durch Klagerücknahme oder beiderseitige Erledigungserklärung beenden und sich anstelle eines Prozessvergleiches für einen Vollstreckungstitel in Form eines Anwaltsvergleichs gemäß \ 794 Abs. 1 Nr. 4b in Verbindung mit $\iint 796 a$ bis 796c ZPO, einer notariellen Urkunde gemäß $\int 794$ Abs. 1 Nr. 5 ZPO oder eines Schiedsspruchs mit vereinbartem Wortlaut ${ }^{198}$ gemäß \ 794 Abs. 1 Nr. 4a in Verbindung mit $\iint 1053,1060$ ZPO entscheiden. Zusätzlich zu den ohnehin schon anfallenden Gerichtskosten würden dann jedoch weitere Kosten für Abschluss bzw. Vollstreckbarerklärung des jeweiligen Vollstreckungstitels entstehen. ${ }^{199}$

\section{bb) Verwaltungs- und sozialgerichtliches Verfahren}

96 Im verwaltungsgerichtlichen Verfahren erhalten die Parteien mit einem gemäß \ 106 VwGO geschlossenen gerichtlichen Vergleich gemäß \ 168 Abs. 1 Nr. 3 VwGO ebenfalls unmittelbar einen Vollstreckungstitel. Gleiches gilt gemäß \ 199 Abs. 1 Nr. 3 SGG im sozialgerichtlichen Verfahren für einen gemäß \101 SGG geschlossenen Prozessvergleich. In der Praxis der gerichtsnahen Mediation am Verwaltungs- und am Sozialgericht Hannover wird allerdings ganz überwiegend auf den Abschluss eines Prozessvergleichs und damit auf einen Vollstreckungstitel verzichtet und darauf vertraut, dass die Beteiligten sich bereits durch den Abschluss des materiell-rechtlichen Vertrages hinreichend gebunden sehen.

\footnotetext{
196 Hierzu Proposal for a Directive of the European Parliament and the Council on certain aspects of Mediation in Civil and Commercial Matters of 22.10.2004; 2004/0251 (COD); COM (2004) 718 final; abrufbar unter: http://europa.eu.int/eur-lex/lex/LexUriServ/site/en/com/2004/com2004_0718en01.pdf.

197 Commission Staff Working Paper, Annex to the proposed mediation directive, 22.10.2004, SEC (2004) 1314, S. 5, abrufbar unter: http://europa.eu.int/comm/justice_home/ejn/adr/adr_ec_annex_mediation_en.pdf.

198 Ein Schiedsspruch mit vereinbartem Wortlaut bietet sich wegen seiner praktisch weltweiten Vollstreckbarkeit insbesondere in Streitigkeiten mit internationalem Bezug an.

199 Zum Anwaltsvergleich: Nerlich, NJW 1997, 416 ff.; zur notariellem Schlichtungsverfahren und notarieller Beurkundung: K.-R. Wagner, BB 1997, 53 ff.; zum Schiedsspruch mit vereinbartem Wortlaut, Eidenmüller, in: Breidenbach/Coester-Waltjen/Heß/Nelle/Wolf, Konsensuale Streitbeilegung, S. 45, 83 ff.; Lörcher, in: Haft/Schlieffen, Handbuch Mediation, S. 747, 755 ff.
} 


\section{Gefahr von Rechtsverlusten während des Mediationsverfahrens}

Entscheiden sich die Parteien für die Durchführung einer gerichtsnahen Mediation, so ist die Sache bereits rechtshängig gemacht, für die Dauer der gerichtsnahen Mediation wird dann das Ruhen des gerichtlichen Verfahrens entsprechend $\$ \$ 278$ Abs. 5 Satz 3, 251 ZPO angeordnet.

Für den Bereich der vorgerichtlichen Mediation könnten sich unter Umständen Gefahren daraus ergeben, dass mit der Durchführung eines Mediationsverfahrens unter Umständen der Verlust von Rechtspositionen verbunden sein kann. Nachfolgend sollen daher mögliche mit der Durchführung eines Mediationsverfahrens verbundene Risiken, namentlich durch Zeitablauf drohende Rechtsverluste, der Verlust von mit der Rechtshängigkeit der Sache verbundenen Privilegien sowie die Gefahr der Verschiebung von Rechts- und Vermögenspositionen, erörtert und im Hinblick auf ihre Relevanz auch im Bereich der gerichtsnahen Mediation auch mit Blick auf die Einbeziehung über den ursprünglichen Streitgegenstand hinausgehende Ansprüche rechtlich untersucht werden, um so die Vorteile der gerichtsnahen Mediation unter den derzeitigen rechtlichen Rahmenbedingungen für die Parteien herausarbeiten zu können.

\section{a) Durch Zeitablauf drohende Rechtsverluste}

\section{aa) Verjährung}

Vor Inkrafttreten des Schuldrechtsmodernisierungsgesetzes am 1. Januar 2002 drohte den Parteien stets die Verjährung ihrer jeweiligen Forderungen während der Dauer des Mediationsverfahrens. ${ }^{200}$ Die neu eingeführte Regelung des $₫ 203$ S. 1 BGB hat diese Gefahr nun beseitigt: Solange zwischen dem Schuldner und dem Gläubiger Verhandlungen über den Anspruch oder die den Anspruch begründenden Umstände schweben, ist die Verjährung gehemmt. Bei der (gerichtsnahen) Mediation handelt es sich um eine Form der - drittunterstützten - Verhandlung, so dass gemäß $\ 203$ S. 1 BGB für die Parteien einer (gerichtsnahen) Mediation ganz unabhängig von der Rechtshängigkeit der Sache stets ein lückenloser Schutz vor einer Verjährung ihrer Forderungen während der Dauer des Mediationsverfahrens besteht. Darüber hinaus ordnet $₫ 203$ S. 2 BGB für die Zeit nach dem Ende der Verhandlungen eine Ablaufhemmung an, ${ }^{201}$ so dass auch bei einem

\footnotetext{
200 Zur alten Rechtslage und der Gefahr des Verjährungseintritts während laufender Mediationsverhandlungen: Eidenmüller, in:Breidenbach/Coester-Waltjen/Heß/Nelle/Wolf, Konsensuale Streitbeilegung, 2001, S. 45, 65; G. Wagner, NJW 2001, 182.

201 Spindler, in: Bamberger/Roth, BGB, Band 1, § 203 Rn. 7.
} 
überraschenden Abbruch der Mediationsverhandlung die Gefahr eines Verjährungseintritts nicht besteht. ${ }^{202}$

100 Als weitere Hemmungstatbestände kommen die \$S 204 Abs. 1 Nr.4, 207 und 209 BGB in Betracht. Gemäß \204 Abs. 1 Nr. 4 wird die Verjährung „,durch die Veranlassung der Bekanntgabe eines Güteantrags, der bei einer durch die Landesjustizverwaltung eingerichteten oder anerkannten Gütestelle oder, wenn die Parteien den Einigungsversuch einvernehmlich unternehmen, bei einer sonstige Gütestelle, die Streitbeilegungen betreibt, eingereicht ist" gehemmt. \207 BGB regelt die Hemmung der Verjährung aus familiären und ähnlichen Gründen. \209 enthält eine Ablaufhemmung in Nachlassfällen. Wegen \203 S. 1 BGB ist ein Rückgriff auf diese spezielleren Hemmungstatbestände jedoch regelmäßig nicht erforderlich.

\section{bb) Ablauf von Ausschlussfristen}

\section{(1) Geltendes Recht}

101 Aufgrund der von den Parteien für die Dauer eines Mediationsverfahrens getroffenen Vereinbarung, keine (gerichtlichen) Schritte zur Durchsetzung ihrer Ansprüche einzuleiten, besteht bei der außergerichtlichen Mediation die Gefahr eines Rechtsverlustes insoweit, ${ }^{203}$ als gesetzliche Ausschlussfristen für die Geltendmachung von Ansprüchen regelmäßig nur durch die Erhebung einer zulässigen Klage gewahrt werden können. ${ }^{204}$ Den Konfliktparteien einer gerichtsnahen Mediation droht diese Gefahr in den meisten Fällen jedoch nicht, da das Mediationsverfahren erst nach Rechtshängigkeit einer Klage über $\int 278$ Abs. 5 S. 2 und 3 ZPO initiiert wird und etwaige von Ausschlussfristen bedrohte Ansprüche damit bereits wirksam geltend gemacht sind.

102 Fraglich ist jedoch, ob sich die Parteien auch in den Fällen, in denen nicht zum Gegenstand der ursprünglichen Klage gehörende Ansprüche in die Mediationsverhandlungen einbezogen werden, vor dem Verlust von Rechten infolge eines Ablaufs von Ausschlussfristen schützen können: Den Verjährungsvorschriften entsprechende Hemmungstatbestände für Ausschlussfristen sind gesetzlich jedenfalls nicht vorgesehen, eine analoge Anwendung der Verjährungsvorschriften

\footnotetext{
202 Heß/Sharma, in: Haft/v. Schlieffen, Handbuch Mediation, S. 675, 700; G. Wagner, ZKM 2002, 103, $107 \mathrm{f}$.

203 Hacke, Der ADR-Vertrag, S.139.

${ }^{204}$ Grothe, in: MünchKommBGB, $\$ 194$ Rn. 8 f.; siehe in diesem Zusammenhang die Ausführungen zur Möglichkeit vertraglicher Regelungen im Bereich vor- und außergerichtlicher Streitbeilegungsformen bei Hacke, Der ADR-Vertrag, S. 140 f.
} 
kommt auf Grund der funktionellen Unterschiede zwischen Verjährungs- und Ausschlussfristen nicht in Betracht ${ }^{205}$ und auch eine vertragliche Verlängerung der Ausschlussfristen wird vom Schutzzweck der Ausschlussfristen verboten. ${ }^{206}$ Denn anders als die Verjährungsfristen dienen Ausschlussfristen nicht in erster Linie dem Schuldnerschutz, sondern der Herstellung von Rechtssicherheit und Rechtsklarheit. ${ }^{207}$

Allerdings kann einer Partei, die sich auf den Ablauf einer Ausschlussfrist beruft, die Einrede der unzulässigen Rechtsausübung gemäß \242 BGB entgegen gehalten werden, wenn sie zuvor durch ihre Bereitschaft zum Versuch einer einvernehmlichen Streitbeilegung das Vertrauen der anderen Partei in den wechselseitigen Verzicht auf die Geltendmachung von Rechten erweckt hat. ${ }^{208}$ Denn der Schuldner handelt treuwidrig, wenn er den Ablauf der Ausschlussfrist dadurch mit veranlasst hat, dass er den Eindruck erweckt hat, er werde sich nicht auf den Fristablauf berufen, und der Gläubiger infolge dessen untätig geblieben ist. ${ }^{209} \mathrm{Ob}$ es sich um außergerichtlich oder im Rahmen der gerichtsnahen Mediation durchgeführte Mediationsverfahren handelt, macht dabei keinen Unterschied.

\section{(2) Rechtspolitische Handlungsempfehlung}

Obwohl sich folglich die Parteien mit $\int 242$ BGB gegen Rechtsverluste durch den

Ablauf von Ausschlussfristen schützen können, empfiehlt sich zur Klarstellung und als positives Signal für die Parteien eine gesetzliche Regelung, die auch für Ausschlussfristen eine Fristhemmung für die Dauer von (Mediations)Verhandlungen vorsieht. ${ }^{210}$ Als Beispiel für eine solche Regelung kann \ 22 des am 1. Mai 2004 in Österreich in Kraft getretenen Zivilrechts-MediationsGesetzes $^{211}$ genannt werden. Nach dieser Vorschrift hemmt eine Mediation den Anfang und den Fortlauf der Verjährung sowie sonstiger Fristen zur Geltendmachung der von den Mediationsverhandlungen betroffenen Ansprüche und Rechte,

\footnotetext{
205 Peters, in: Staudinger, BGB, JS 164-240, Vor $\int 194$ Rn. 15.

206 OLG Celle WM 1975, 652, 654; Henrich, in: Bamberger/Roth, BGB, Band 1, § 194 Rn. 4.

207 Peters, in: Staudinger, BGB, SS 164-240, Vor \ 194 Rn. 13.

208 Hacke, Der ADR-Vertrag, S. 141; Böttcher/Laskawy, Der Betrieb 2004, 1247, 1251.

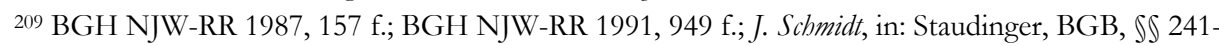
243, \ 242 Rn. 613 f.; Grothe, in: MünchKommBGB, Band 1, \ 194 Rn. 13.

210 So auch Art. 7 des Entwurfs einer europäischen Mediationsrichtlinie, abgedruckt in ZKM 2004, 149 f.; Volkmann, Mediation im Zivilprozess. Rechtliche Rahmenbedingungen für ein gerichtsinternes Mediationsangebot, S. $86 \mathrm{f}$.

211 Ausführlich zum Zivilrechts-Mediations-Gesetz Ferz/Filler, Mediation. Gesetzestexte und Kommentar; Pruckner, Recht der Mediation; Oberhammer/Domej, ZKM 2003, 144 f.
} 
wenn der Mediator in die offizielle Mediatorenliste des österreichischen Bundesministeriums für Justiz eingetragen ist. ${ }^{212}$

\section{b) Verlust von mit der Rechtshängigkeit der Sache verbundenen Rechtsvorteilen}

\section{aa) Materiell-rechtliche Wirkungen der Rechtshängigkeit}

105 Im Bereich der vorgerichtlichen Mediation muss der Gläubiger wegen des in der Mediationsvereinbarung regelmäßig vereinbarten zeitweiligen Klageverzichts auf die durch die Rechtshängigkeit einer Sache herbeigeführten für ihn positiven materiell-rechtlichen Wirkungen, wie beispielsweise den Eintritt des Verzuges gemäß \ 286 Abs. 1 S. 2 BGB, den Zinsanspruch gemäß \ 291 BGB sowie Haftungsverschärfungen für den Schuldner gemäß $\iint$ 292, 818 Abs. 4, 987 Abs. 2, 988, 989, 991, 994 Abs. 2, 996, 1007 Abs. 2 und 2023 BGB213, verzichten. ${ }^{214}$ Im Bereich der gerichtsnahen Mediation besteht dieser Nachteil dagegen in den meisten Fällen nicht, da das Mediationsverfahren gemäß \ 278 Abs. 5 S. 2 und 3 ZPO während des gerichtlichen Verfahrens und damit erst nach Rechtshängigkeit der Sache eingeleitet wird, so dass der Gläubiger hier regelmäßig von den für den Kläger positiven materiell-rechtlichen Wirkungen der Rechtshängigkeit profitieren kann. Etwas anderes gilt nur im Hinblick auf die Ansprüche, die nachträglich in die Mediationsverhandlungen einbezogen werden, ohne bereits zum Gegenstand der Klage zu gehören. ${ }^{215}$

\section{bb) Prozesstaktische Vorteile}

106 Keinesfalls besteht im Bereich der gerichtsnahen Mediation dagegen die Möglichkeit einer Streitverkündung gegenüber am Prozess nicht beteiligten Dritten. Davon ausgehend, dass der Ausgang des konkreten Konflikts Auswirkungen auf ein etwaiges Vorgehen gegen Außenstehende bzw. auf die eigene Verteidigungsposition gegenüber rechtlichen Angriffen eines Dritten haben kann, können die Konfliktparteien ein Interesse daran haben, im Wege einer Streitverkündung die Ne-

\footnotetext{
${ }^{212}$ Kritisch zu dem Listensystem nach dem österreichischen Zivilrechts-Mediations-Gesetz Köper, ZKM 2004, $161 \mathrm{ff}$.

213 Wegen der weiteren materiell-rechtlichen Wirkungen der Klageerhebung, siehe Greger, in: Zöller, ZPO, Vor $\$ 253$ Rn. 26.

${ }^{214}$ Hacke, Der ADR-Vertrag, S. 146 f.

215 Volkmann, Mediation im Zivilprozess. Rechtliche Rahmenbedingungen für ein gerichtsinternes Mediationsangebot, S. 87 f.
} 
beninterventionswirkung gemäß $\iint 74$ Abs. 3, 68 ZPO herbeizuführen. ${ }^{216}$ Da die Nebeninterventionswirkung nur durch rechtskräftiges Sachurteil, nicht aber durch einen Prozessvergleich herbeigeführt werden kann, ${ }^{217}$ bleibt den Parteien im Bereich der gerichtsnahen Mediation diese Möglichkeit jedoch verschlossen. ${ }^{218}$

\section{c) Gefahr der Verschiebung von Rechts- und Vermögenspositionen}

In tatsächlicher Hinsicht droht schließlich die Gefahr, dass eine Partei das Mediationsverfahren dazu nutzt, die zu verhandelnden Rechtspositionen und Vermögenswerte zu ihren Gunsten zu verschieben und dadurch dem Zugriff der anderen Partei zu entziehen. ${ }^{219}$ Im Rahmen einer vorgerichtlichen, also einer nicht nach \$ 278 Abs. 5 S.2 ZPO veranlassten außergerichtlichen Mediation verpflichten sich die Parteien in ihrer Mediationsvereinbarung grundsätzlich zur Unterlassung sämtlicher Prozesshandlungen während der Dauer des Mediationsverfahrens. Zum Schutz vor einer Verschiebung von Rechts- und Vermögenspositionen empfiehlt es sich daher, Maßnahmen des einstweiligen Rechtsschutzes von der in der Mediationsvereinbarung geregelten Unterlassungsverpflichtung auszunehmen. ${ }^{220}$ Bei der nach \278 Abs. 5 S. 2 ZPO gerichtsnahen, also sowohl gerichtsinternen als auch außergerichtlichen, Mediation ist eine solche Vereinbarung zur Unterlassung sämtlicher Prozesshandlungen während der Dauer des Mediationsverfahrens nicht erforderlich. Hier wurde zum einen bereits Klage erhoben, zum anderen sind aufgrund des in $\iint 278$ Abs. 5 Satz 3, 251 ZPO entsprechend angeordneten Ruhens des Verfahrens ohnehin Prozesshandlungen in Ansehung der Hauptsache, welche eine Partei während des Stillstands vornimmt, gem. \249 Abs. 2 ZPO gegenüber dem Gegner unwirksam.221 Sofern die Mediationsvereinbarung dennoch die Unterlassung prozessualer Maßnahmen regelt, empfiehlt sich in jedem Fall auch die Aufnahme der beschriebenen Rückausnahme für Anträge im einstweiligen Rechtsschutz, um ggf. Rechtsverluste durch die Verschiebung von Rechts- oder Vermögenspositionen verhindern zu können. Denn Anträge im Verfahren des einstweiligen Rechtsschutzes werden zwar von \ 249 Abs. 2 ZPO

\footnotetext{
216 Hacke, Der ADR-Vertrag, S. 146.

217 BGH VersR 1958, 762, 763; BGH NJW 1969, 1480, 1481; Musielak, ZPO, I 68 Rn. 2; Vollkommer, in Zöller, ZPO, \68 Rn. 4.

218 Volkmann, Mediation im Zivilprozess. Rechtliche Rahmenbedingungen für ein gerichtsinternes Mediationsangebot, S. 88. Zu Überlegungen zur Anreizsetzung durch die Einbeziehung Dritter siehe unten Rn. 353.

219 Hacke, Der ADR-Vertrag, S. 142.

220 Hacke, Der ADR-Vertrag, S. 144.

221 Zur entsprechenden Anwendung des $\int 249$ Abs. 1 und 2 ZPO auf das Ruhen des Verfahrens nach \ 251 ZPO Greger, in Zöller, ZPO, \ 251 Rn. 1.
} 
nicht erfasst, da sie einen von der Hauptsache zu unterscheidenden Streitgegenstand betreffen; sie können daher auch während des Ruhens des Verfahrens gestellt werden. ${ }^{222}$ Anders wäre dies hingegen bei einer unbeschränkt formulierten Unterlassungsvereinbarung. Bezieht sich die Unterlassungsvereinbarung auch auf Prozesshandlungen in Bezug auf die später verschobenen Vermögensgegenstände, welche nicht mit dem Streitgegenstand des ruhenden Hauptsacheverfahrens identisch sind, ist auch insoweit eine Rückausnahme für Maßnahmen im einstweiligen Rechtsschutz empfehlenswert.

\section{Vertraulichkeit in der gerichtsnahen Mediation}

108 Die Parteien haben häufig ein anerkennenswertes Interesse daran, dass ihre Auseinandersetzung nicht Thema einer unsensiblen medialen Berichterstattung wird, was bei einem normalen gerichtlichen Verfahren wegen des Grundsatzes der Öffentlichkeit gem. \169 GVG nicht gewährleistet ist. Eine Rechtfertigung für die Vertraulichkeit der Mediation liegt aber auch im Mediationsverfahren selbst: Der Erfolg einer Mediation hängt nämlich ganz wesentlich davon ab, dass die Parteien offen miteinander kommunizieren, und zwar nicht nur im Hinblick auf ihre Vergleichsbereitschaft, sondern auch bezüglich der hinter ihren jeweiligen Positionen verborgenen Interessen und Bedürfnisse. ${ }^{223} \mathrm{Da}$ es jedoch keine Garantie dafür gibt, dass ein Mediationsverfahren auch tatsächlich mit einer gütlichen Einigung endet, besteht für die Parteien die Gefahr eines Missbrauchs der in der Mediation mitgeteilten vertraulichen Informationen, insbesondere im geschäftlichen Verkehr, aber auch im Bereich ihrer privaten Beziehungen. ${ }^{224} \mathrm{Zu}$ befürchten ist insbesondere ein strategisches Verhalten in dem Sinne, dass ohne echte Einigungsabsicht die systemimmanente Offenheit in der Mediation als taktisches Mittel zum Ausspionieren der anderen Partei genutzt wird und sich die Parteien dadurch Nachteilen im Hinblick auf den Fortgang des gerichtlichen Verfahrens aussetzen. 225 Wesentliche Voraussetzung für eine erfolgreiche Mediation ist daher der Schutz der Vertraulichkeit der in der Mediation offenbarten Informationen.

222 Greger, in Zöller, ZPO, \ 249 Rn. 3 mit Vor \ 239 Rn. 8.

223 Gray, 36 Osgoode Hall Law Journal 667, 671 (1998).

224 Gray, 36 Osgoode Hall Law Journal 667, 671 (1998); Deason, Public Law and Legal Theory Working Paper Series No. 22/ Center for Law, Policy and Social Science Working Paper Series No. 15, 14.

${ }^{225}$ Für die außergerichtiche Mediation: Weigand, BB 1996, 2106, 2108; Casper/Risse, ZIP 2000, 437, 440; Köper, Die Rolle des Rechts im Mediationsverfahren, S. 30. 


\section{a) Materiell-rechtliche Regelungen zum Schutz der Vertraulichkeit in der Mediation}

Spezielle gesetzliche Bestimmungen zum Schutz der Vertraulichkeit in der Mediation existieren nicht. Zu nennen sind in diesem Zusammenhang jedoch die allgemeinen, an die berufliche Tätigkeit anknüpfenden Vorschriften, die eine Verschwiegenheitspflicht des Mediators bezüglich ihm in der Mediation anvertrauter oder bekannt gewordener Informationen begründen können. ${ }^{226}$ Für den Richtermediator ergibt sich diese Pflicht zunächst aus seiner allgemeinen Pflicht zur Amtsverschwiegenheit gemäß \68 Abs. 1 NBG. Darüber hinaus kann sich eine Verschwiegenheitspflicht sowohl für Richtermediatoren als auch für die im Rahmen der gerichtsnahen Mediation tätigen Psychologen aus \203 StGB ergeben.227 Eine Geheimhaltungspflicht der an der Mediation beteiligten Personen wird darüber hinaus regelmäßig auch vertraglich vereinbart werden, insbesondere mit Blick darauf, dass die Vertraulichkeit nicht nur durch den Mediator, sondern auch durch die Konfliktparteien sowie an der Mediation beteiligte Dritte zu wahren ist.

\section{b) Schutz der Vertraulichkeit im nachfolgenden Prozess}

Die materiell-rechtliche Verpflichtung der Beteiligten, die in der Mediation offenbarten Informationen vertraulich zu behandeln, gewährleistet jedoch nicht zugleich auch den Schutz der Vertraulichkeit in einem nachfolgenden Prozess. Hierzu bedarf es vielmehr einer prozessualen Regelung:

\section{aa) Gesetzliche Bestimmungen}

\section{(1) Zivilprozess}

Als gesetzliche Bestimmung zum Schutz der Vertraulichkeit der Mediationsverhandlungen kommt allein ein gesetzlich geregeltes Zeugnisverweigerungsrecht der im Rahmen der gerichtsnahen Mediation tätigen Mediatoren gemäß $\iint 383$ ff. ZPO in Betracht. \376 ZPO kann insoweit keinen verlässlichen Schutz vor einer Aussage des Richtermediators in einem nachfolgenden Gerichtsverfahren bieten, als eine Aussagegenehmigung zwar im Hinblick auf die Bedeutung des Vertrau-

\footnotetext{
226 Näher dazu Volkmann, Mediation im Zivilprozess. Rechtliche Rahmenbedingungen für ein gerichtsinternes Mediationsangebot, S. 119.

227 Für Richtermediatoren ergibt sich die Schweigepflicht aus $\ 203$ Abs. 2 Nr. 1, für Psychologen aus $\int 203$ Abs. 1 Nr. 2 StGB; ausführlich zur gesetzlich begründeten Schweigepflicht des Psychologen C. Hartmann, in: Haft/Schlieffen, Handbuch Mediation, S. 712, 718.
} 
lichkeitsschutzes für das Mediationsverfahren verweigert werden kann, 228 es jedoch keineswegs garantiert ist, dass eine solche Entscheidung auch einer Überprüfung durch die Verwaltungsgerichte standhalten würde. ${ }^{229}$

112 Ein Zeugnisverweigerungsrecht ergibt sich dagegen unabhängig von ihrem beruflichen Hintergrund als Richter oder Psychologen gemäß \383 Abs. 1 Nr. 6 bereits aus der Mediatorentätigkeit selbst. \383 Abs. 1 Nr. 6 ZPO gesteht denjenigen Personen ein Zeugnisverweigerungsrecht zu, ,denen kraft ibres Amtes, Standes oder Gewerbes Tatsachen anvertraut sind, deren Geheimbaltung durch ibre Natur oder durch gesetzliche Vorschrift geboten ist, in betreff der Tatsachen, auf welche die Verpflicbtung zur Verschwiegenheit sich bezieht". Neben der Möglichkeit eines Zeugnisverweigerungsrechts der in der gerichtsnahen Mediation tätigen Mediatoren aufgrund ihrer gesetzlich begründeten Schweigepflicht ${ }^{230} \mathrm{kann}$ sich für Mediatoren ein Zeugnisverweigerungsrecht daher auch daraus ergeben, dass ihre Verschwiegenheit bezüglich während des Mediationsverfahrens bekannt gewordener Tatsachen kraft Natur der Sache geboten ist. Was kraft Natur der Sache geheim zu halten ist, ist mit Rücksicht auf die Verkehrssitte und die berechtigten Erwartungen der vertrauenden Personen zu beurteilen. ${ }^{231}$

113 Die Vertraulichkeit der Mediationsverhandlungen gehört zu den grundlegenden Prinzipien des Mediationsverfahrens. Demnach entspricht der vertrauliche Umgang des Mediators mit ihm in der Mediation bekanntgewordenen Informationen nicht nur den berechtigten Erwartungen der Parteien. ${ }^{232}$ Es hat sich zudem inzwischen auch eine entsprechende Verkehrssitte herausgebildet:233 Die vertrauliche Behandlung von in der Mediation bekannt gewordenen Informationen ist typi-

${ }^{228}$ Ein Grund für die Verweigerung einer Aussagegenehmigung ist es zum Beispiel, wenn die Arbeit der Behörde von einer besonderen Vertrauensatmosphäre geprägt ist, die durch eine Zeugenaussage über vertrauliche Gespräche beeinträchtigt würde, siehe dazu Sommer/Konert/Sommer, NBG, \ 69 Rn. 4; Zängl, in: Fürst, GKÖD, Band 1, \ 62 BBG Rn. 11; Löer, ZKM 2006, 4.

${ }^{229}$ Siehe dazu BVerwGE 18, 58 ff. Ausführlich zum Ausschluss einer Vernehmung des Richermediators gemäß \376 ZPO Volkmann, Mediation im Zivilprozess. Rechtliche Rahmenbedingungen für ein gerichtsinternes Mediationsangebot, S. $125 \mathrm{ff}$.

230 Musielak, ZPO, \ 376 Rn. 6, \383 Rn. 6; Chr. Berger, in Stein/Jonas, ZPO, Band 4/2, 』383 Rn. $21,71$.

231 Damrau, in: MünchKommZPO, Band 2, \ 383 Rn. 39; Chr. Berger, in: Stein/Jonas, \ 383 Rn. 85, 91; Thomas/Putzo, ZPO, \383 Rn. 7.

232 Eidenmüller, in:Breidenbach/Coester-Waltjen/Heß/Nelle/Wolf, Konsensuale Streitbeilegung, S. $45,65$.

233 Eidenmüller, in:Breidenbach/Coester-Waltjen/Heß/Nelle/Wolf, Konsensuale Streitbeilegung, S. 45, 65; C. Hartmann, in: Haft/Schlieffen, Handbuch Mediation, S. 712, 731; Mähler/Mähler, ZKM $2001,4,7$. 
scher Bestandteil von Verfahrensordnungen und Richtlinien zum Mediationsverfahren 234 und insbesondere auch eines von der EU-Kommission in Zusammenarbeit mit Vertretern verschiedener europäischer Konfliktbeilegungsorganisationen entwickelten europäischen Verhaltenskodexes für Mediatoren ${ }^{235}$ und des Vorentwurfs zu einer europäischen Mediationsrichtlinie.236 Ein kraft Natur der Sache bestehendes Zeugnisverweigerungsrecht des Mediators gemäß \383 Abs. 1 Nr. 6 $\mathrm{ZPO}$ ist daher anzuerkennen. ${ }^{237}$

\section{(2) Verwaltungs- und sozialgerichtliches Verfahren}

Die Ausführungen zu einem Zeugnisverweigerungsrecht des Mediators im Bereich eines zivilgerichtlichen Mediationsangebotes gelten entsprechend für die gerichtsnahe Mediation an Verwaltungs- und Sozialgerichten. Für die Beweisaufnahme im verwaltungsgerichtlichen Verfahren erklärt $\ 98$ VwGO die $\$ \int 358$ bis 444 und 450 bis 494 ZPO für entsprechend anwendbar, so dass dem am Verwaltungsgericht tätigen Richtermediator die gleichen Zeugnisverweigerungsmöglichkeiten zustehen, wie dem Richtermediator am Amts- oder am Landgericht. Für das sozialgerichtliche Verfahren verweist $₫ 118$ Abs. 1 SGG auf die $\$ ₫ 383,384$ $\mathrm{ZPO}$, so dass auch für den am Sozialgericht tätigen Richtermediator ein Zeugnisverweigerungsrecht gemäß \383 Abs. 1 Nr. 6 ZPO anzuerkennen ist.

${ }^{234}$ \6 der Verfahrensordnung der Gesellschaft für Wirtschaftsmediation und Konfliktmanagement e.V. (gwmk), nachzulesen bei C. Hartmann, in: Haft/Schlieffen, Handbuch Mediation, S. 712, 739; Richtlinien der Bundes-Arbeitsgemeinschaft für Familien-Mediation (BAFM), Nr. II 5 II/1, nachzulesen bei Breidenbach/Henssler, Mediation für Juristen, S. 123, 125.

235 European Code of Conduct for Mediators, 4. Confidentiality, abgedruckt in: ZKM 2004, $148 \mathrm{f}$.

236 Hierzu Proposal for a Directive of the European Parliament and the Council on certain aspects of Mediation in Civil and Commercial Matters of 22.10.2004; 2004/0251 (COD); COM (2004) 718 final (siehe oben. Fn. 7).

237 Eidenmüller, in: Breidenbach/Coester-Waltjen/Heß/Nelle/Wolf, Konsensuale Streitbeilegung, S. 45, 65; C. Hartmann, in: Haft/Schlieffen Handbuch Mediation, S. 712, 731; Mäbler/Mäbler, ZKM 2001, 4, 7; Eckart/Dendorfer, MDR 2001, 786, 790; Greger, in: Zöller, ZPO, \383 Rn. 20; wohl auch P. Hartmann, in: Baumbach/Lauterbach/Albers/P. Hartmann, ZPO, \$383 Rn. 17; a.A. Musielak, ZPO, \376 Rn. 6, §383 Rn. 6 ohne weitere Begründung mit Hinweis auf Groth/v. Bubnoff, NJW 2001, 338. 


\section{bb) Erforderlichkeit, Möglichkeiten und Grenzen einer vertraglichen Regelung zum Schutz der Vertraulichkeit im nachfolgenden Prozess}

\section{(1) Zivilprozess}

\section{(a) Geltendes Recht}

115 Das Zeugnisverweigerungsrecht betrifft jedoch immer nur den Mediator. Die Parteien können dagegen in der Mediation offenbarte vertrauliche Informationen zum Inhalt ihres Sachvortrags machen und darüber hinaus zum Nachweis der Informationen andere Beweismittel als den Zeugenbeweis benennen. ${ }^{238}$ Zudem beinhaltet das Zeugnisverweigerungsrecht des Mediators keine prozessuale Pflicht zur Aussageverweigerung, so dass auch in dieser Hinsicht kein vollständiger Schutz der Vertraulichkeit besteht. ${ }^{239}$ Es besteht daher ein Bedürfnis die Vertraulichkeit der Mediationsverhandlungen im nachfolgenden Prozess vertraglich sicher zu stellen.

116 Jedenfalls für den Zivilprozess wird die Zulässigkeit vertraglicher Vereinbarungen der Parteien zur Vornahme oder Unterlassung von Prozesshandlungen ${ }^{240}$ und insbesondere von Vereinbarungen sowohl über den Vortrag bzw. Nicht-Vortrag bestimmter Tatsachen als auch über die Benennung bestimmter Beweismittel auch von der herrschenden Meinung anerkannt. ${ }^{241}$ Die grundsätzliche Zulässigkeit derartiger Vereinbarungen ergibt sich aus dem dem Zivilprozess zugrunde liegenden Dispositionsgrundsatz und der Verhandlungsmaxime.242 Soweit die Zivilprozessordnung den Parteien die Ausübung bestimmter Rechte, wie den Vortrag von Tatsachen oder den Antritt von Beweisen, freistellt, ist es auch zulässig, hierüber

238 G. Wagner, NJW 2001, 1398.

239 G. Wagner, NJW 2001, 1398.

${ }^{240}$ Lüke, in: MünchKommZPO, Band 1, Einl. Rn. 285; Musielak, ZPO, Einl. Rn. 67; Leipold, in: Stein/Jonas, ZPO, Band 2, Vor \128 Rn. 237; Greger, in: Zöller, ZPO, Vor \ 128 Rn. 32; ausführlich G. Wagner, Prozeßverträge - Privatautonomie im Verfahrensrecht.

241 BGHZ 38, 254, 258; BGHZ 109, 19, 28 f; P. Hartmann, in: Baumbach/Lauterbach/Albers/P. Hartmann, ZPO, Einf. \ 284 Rn. 33; Greger, in: Zöller, ZPO, Vor \ 284 Rn. 23; Prütting, in: MünchKommZPO, Band 1, \ 286 Rn. 159; Schlosser, Einverständliches Parteihandeln im Zivilprozess, S. 86 ff.; Thomas/Putzo, ZPO, Vorbem \ 284 Rn. 41; G. Wagner, Prozeßverträge - Privatautonomie im Verfahrensrecht, S. 608 ff.; G. Wagner, NJW 2001, 1399.

${ }^{242}$ C. Hartmann, in: Haft/Schlieffen, Handbuch Mediation, S. 712, 726; Leipold, in: Stein/Jonas, ZPO, Band 2, Vor \ 128 Rn. 236; G. Wagner, NJW 2001, 1399. 
vertragliche Vereinbarungen zu treffen. ${ }^{243}$ Der Ausschluss des Vortrags bestimmter Tatsachen oder der Benennung bestimmter Beweismittel führt dazu, dass ein abredewidriger Sachvortrag oder Beweisantritt vom Gericht im Prozess nicht zu berücksichtigen ist. ${ }^{244}$

Gleichwohl ist jedoch auch die Wirkung eines von den Parteien bezüglich Tatsachenvortrag und Benennung von Beweismitteln geschlossenen Beweisvertrags insoweit beschränkt, als einer vertraglichen Vereinbarung immer auch das Risiko der Unwirksamkeit aus vertragsrechtlichen Gründen innewohnt. ${ }^{245}$ Darüber hinaus kann ein solcher Vertrag immer nur zwischen den am Mediationsverfahren unmittelbar beteiligten Parteien wirken: An der Mediation nicht beteiligte Dritte sind regelmäßig nicht gehindert, den Inhalt des Mediationsverfahrens durch Tatsachenvortrag oder entsprechende Beweisanträge in einen nachfolgenden Prozess einzuführen. ${ }^{246}$ Schließlich ist die Wirkung von Beweismittelverträgen auch insoweit begrenzt, als das Gericht die dem Vertrag unterliegenden Informationen auch ohne Einverständnis der Parteien - wenn auch nur in engen Grenzen - im Wege richterlicher Beweisanordnung in den Prozess einführen kann. ${ }^{247}$ Denn die $\iint 142$, 143, 144, 448, 273 Abs. 2 ZPO stellen auch im Zivilprozess die Beweiserhebung von Amts wegen - außer für den Zeugenbeweis - in das Ermessen des Gerichts.

Zusammenfassend bleibt festzuhalten, dass mit den Mitteln des Vertragsrechts ein umfassender Schutz der Vertraulichkeit der Mediationsverhandlungen im nachfolgenden Gerichtsverfahren nicht hergestellt werden kann.

\section{(b) Rechtspolitische Handlungsempfehlung}

Da folglich mit den Mitteln des Vertragsrechts ein umfassender Schutz der Vertraulichkeit der Mediationsverhandlungen im nachfolgenden Gerichtsverfahren nicht hergestellt werden kann, besteht ein dringendes Bedürfnis eine gesetzliche Regelung des Vertraulichkeitsschutzes in der (gerichtsnahen) Mediation zu schaffen. Um einen umfassenden Schutz der Vertraulichkeit der Mediationsverhandlungen sicherzustellen empfiehlt sich eine gesetzliche Regelung nach dem Vorbild des im Juni 2002 von der United Nation Commission on International Trade Law

\footnotetext{
243 C. Hartmann, in: Haft/Schlieffen, Handbuch Mediation, S. 712, 726; G. Wagner, NJW 2001, 1399.

244 Eckeart/Dendorfer, MDR 2001, 786, 790; G. Wagner, NJW 2001, 1399.

245 Vgl. insoweit G. Wagner, NJW 2001, 1398, 1400 zur Unwirksamkeit von AGB-Klauseln zum Schutz der Vertraulichkeit im Prozess.

246 Hacke, Der ADR-Vertrag, S. 261, 267.

247 Greger, in: Zöller, ZPO, Vor \ 284 Rn. 2; Leipold, in: Stein/Jonas, ZPO, Band 3, \ 286 Rn. 133;

Foerste, in: Musielak, ZPO, \ 286 Rn. 16.
} 
(UNCITRAL) verabschiedeten „Model Law On International Commercial Conciliation". ${ }^{248}$ Dieses fordert in Art. 9 die vertrauliche Behandlung aller in der Mediation offenbarten vertraulichen Informationen und enthält zudem in Art. 10 (1) eine Zeugnisverweigerungspflicht der am Mediationsverfahren Beteiligten sowie jedes Dritten. Damit werden nicht nur die Parteien und der Mediator, sondern auch alle übrigen Personen an einem Missbrauch vertraulicher Informationen gehindert. Im Gegensatz dazu ist der in Art. 6 (1) des von der EU-Kommission veröffentlichten Vorentwurf zu einer europäischen Mediationsrichtline ${ }^{249}$ gemachte Regelungsvorschlag für den Schutz der Vertraulichkeit der Mediationsverhandlungen ebenso unzulänglich wie $\int 18$ des am 1. Mai 2004 in Österreich in Kraft getretenen Zivilrechts-Mediations-Gesetzes:250 In beiden Fällen wird allein die Verschwiegenheit des Mediators sowie seiner Hilfspersonen gefordert und durch ein entsprechendes Beweisverwertungsverbot abgesichert.

120 Eine umfassende gesetzliche Regelung des Vertraulichkeitsschutzes nach dem Vorbild des UNCITRAL-Modellgesetzes sollte, um den individuellen Bedürfnissen der Beteiligten gerecht werden zu können, allerdings dispositiv ausgestaltet sein. ${ }^{251}$ Außerdem muss die Regelung deutlich machen, dass der Schutz der Vertraulichkeit dort seine Grenze findet, wo gesetzliche Offenbarungspflichten die Offenlegung vertraulicher Informationen verlangen ${ }^{252}$ oder die betroffenen Informationen auch auf andere Weise hätten beschafft werden können. Denn eine Mediation könnte sonst dazu missbraucht werden, bestimmte Tatsachen für einen späteren Prozess auszuschließen. ${ }^{253}$

248 Ebenso Duve/Prause, IDR 2004, 126, 131; Volkmann, Mediation im Zivilprozess. Rechtliche Rahmenbedingungen für ein gerichtsinternes Mediationsangebot, S. 135. Das UNCITRAL Model Law On International Commercial Conciliation ist abrufbar unter:

www.uncitral.org/english/texts/arbitration/ml-conc-e.pdf.

${ }^{249}$ Proposal for a directive on certain aspects of mediation in civil and commercial matters, siehe oben Fn. 7; zu den Unzulänglichkeiten der Richtlinie hinsichtlich des Vertrauensschutzes Roth, IDR 2005, 114, $121 \mathrm{f}$.

250 Ausführlich zum gesetzlichen Vertraulichkeitsschutz in Österreich Allmayer-Beck, IDR 2004, 119, 124 f.; allgemein zu dem neuen österreichischen Zivilrechtsmediationsgesetz Ferz/Filler, Mediation. Gesetzestexte und Kommentar.

251 Duve/Prause, IDR 2004, 126, 131.

252 Siehe z.B. $\iint 118,166,338$ HGB, 131 AktG, 51a GmbHG.

253 Eidenmüller, in: Breidenbach/Coester-Waltjen/Heß/Nelle/C. Wolf, Konsensuale Streitbeilegung, S. 45, 66; Duve/Prause, IDR 2004, 126, 127. 


\section{(2) Verwaltungs- und sozialgerichtliches Verfahren}

Anders als im Zivilprozess gilt im verwaltungs- und im sozialgerichtlichen Verfahren der Amtsermittlungsgrundsatz, in dessen Anwendungsbereich die Gerichte an Beweismittelverträge der Parteien nicht gebunden sind ${ }^{254}$ und von Amts wegen Beweis erheben können. Eine Möglichkeit, die Vertraulichkeit der Mediationsverhandlungen durch Abschluss eines Beweismittelvertrages zu sichern, haben die Konfliktparteien damit im verwaltungs- und im sozialgerichtlichen Verfahren noch weniger als im Zivilprozess. Im Hinblick auf das dem Amtsermittlungsgrundsatz zugrundeliegende öffentliche Interesse an der Sachverhaltsaufklärung wird diese Einschränkung der Dispositionsbefugnis der Parteien im Bereich der öffentlich-rechtlichen Gerichtsbarkeiten auch hinzunehmen sein.

Umso mehr ergibt sich für die gerichtsnahe Mediation in diesem Bereich ein Bedürfnis für eine gesetzliche Regelung zum Schutz der Vertraulichkeit der Mediationsverhandlungen. Hinsichtlich der Ausgestaltung einer solchen Regelung wird auf die vorstehenden Ausführungen zum Zivilprozess verwiesen.

\section{Fazit}

Um den Bestand der in der Mediation getroffenen Vereinbarung zu gewährleisten, muss ein Bruch dieser Vereinbarung ineffizient oder gar ausgeschlossen sein. Dies ist im Zivilprozess dadurch möglich, die Einhaltung vertraglich festzuhalten und somit die Durchsetzung der Vereinbarung den Gerichten zugänglich zu machen. Aufgrund des mit einem Rechtsstreit verbundenen Prozessrisikos ist es jedoch darüber hinaus sinnvoll, Maßnahmen zur Sicherung der Einhaltung des Vertrages zu ergreifen, wie zum Beispiel materiell-rechtliche Sanktionen. Zur weiteren Absicherung bietet es sich an, über die Vereinbarung einen Vollstreckungstitel zu bekommen, um sie gegebenenfalls im Wege der Zwangsvollstreckung durchsetzen zu können. Für diese prozessuale Absicherung stehen den Parteien zum einen der Prozessvergleich und zum anderen weitere Vollstreckungstitel, wie zum Beispiel eine notarielle Urkunde, zur Verfügung.

Im Rahmen eines Mediationsverfahrens im verwaltungs- bzw. sozialgerichtlichen Verfahren ist für den Vergleich, welcher ein öffentlich-rechtlicher Vertrag ist, erforderlich, dass es der Verwaltung gestattet ist, den Verfahrensgegenstand durch einen öffentlich-rechtlichen Vertrag zu regeln. Darüber hinaus sind die Grundsätze des Vorrangs und des Vorbehalts des Gesetzes, der Gleichbehandlungsgrundsatz, Form- und Zuständigkeitsgesichtspunkte und eine unter Umständen nötige

${ }^{254}$ G. Wagner, Prozeßverträge, S. 683, 688; Hager, Konflikt und Konsens, S. 118. 
Zustimmung eines betroffenen Dritten zu beachten. Dieser geschlossene Vergleich ist dann gemäß \168 Abs. 1 Nr. VwGO bzw. \199 Abs. 1 Nr. 3 SGG ein Vollstreckungstitel, so dass auch die Durchsetzbarkeit gewährleistet ist.

125 Bezüglich eines etwaigen Rechtsverlustes während des Mediationsverfahrens durch Verjährung besteht für die Parteien aufgrund der Hemmung gemäß $\ 203$ S. 1 BGB keinerlei Gefahr. Auch einen Rechtsverlust durch Ablauf von Ausschlussfristen brauchen die Parteien nicht zu fürchten, da das gerichtsnahe Mediationsverfahren zumeist erst nach Rechtshängigkeit, mit der etwaige Ansprüche bereits wirksam geltend gemacht wurden, initiiert wird. Aus diesem Grund besteht auch keine Gefahr für die Parteien, dass auf materiell-rechtliche Ansprüche, wie beispielsweise der Eintritt des Verzuges gemäß \286 Abs. 1 S. 2 BGB, verzichtet werden muss, was bei einer außergerichtlichen Mediation aufgrund des vereinbarten Klageverzichts regelmäßig der Fall ist.

126 Für den Fall, dass ein Anspruch noch nicht rechtshängig ist und die gegnerische Partei sich nun auf den Ablauf von Ausschlussfristen beruft, kann ihr die Einrede der unzulässigen Rechtsausübung gemäß $\ 242$ BGB entgegengehalten werden. Darüber hinaus wäre es wünschenswert, wenn eine gesetzliche Regelung geschaffen würde, die, ähnlich wie schon bei der Verjährung, den Ablauf von Ausschlussfristen während eines Mediationsverfahrens hemmt.

127 Die Möglichkeit einer Streitverkündung besteht in einem Mediationsverfahren im Gegensatz zu einem normalen streitigen Verfahren nicht, da die Nebeninterventionswirkung nur durch rechtskräftiges Sachurteil, nicht hingegen durch einen Prozessvergleich herbeigeführt werden kann.

128 Der Grundsatz der Vertraulichkeit wird auf Seiten des Richtermediators durch \ 68 Abs. 1 NBG gewährleistet. Darüber hinaus kann sich für ihn und für an der Mediation beteiligte Psychologen die Verschwiegenheitspflicht aus $\ 203$ StGB ergeben. Eine Verschwiegenheitspflicht aller weiteren an der Mediation beteiligten Personen ist nicht gesetzlich geregelt und ist insofern vertraglich festzulegen. ${ }^{255}$

129 Für einen gegebenenfalls nachfolgenden Prozess ergibt sich für den Richtermediator und für die an einer Mediation beteiligten Psychologen ein Zeugnisverweigerungsrecht aus $₫ 383$ Abs. 1 Nr. 6 ZPO bzw. \98 VwGO und $\ 118$ Abs. 1 SGG, die auf $\int 383$ Abs. 1 Nr. 6 ZPO verweisen. Darüber hinaus ist die vertrauliche Behandlung von in der Mediation bekannt gewordenen Informationen Bestandteil von Verfahrensordnungen und Richtlinien zum Mediationsverfahren. Ferner ist

${ }^{255}$ Zur rechtspolitischen Forderung einer gesetzlichen Regelung sogleich. 
die Vertraulichkeit Bestandteil eines von der EU-Kommission und Vertretern verschiedener europäischer Konfliktbeilegungsorganisationen entwickelten europäischen Verhaltenskodexes für Mediatoren und des Vorentwurfs einer europäischen Mediationsrichtlinie.

Da das Zeugnisverweigerungsrecht jedoch nur dem Mediator und nicht den Parteien zusteht und der Mediator prozessual nicht gezwungen ist, zu schweigen, besteht ein Bedürfnis, die Vertraulichkeit im nachfolgenden Prozess zu gewährleisten. Im Zivilprozess ist dies, anders als im verwaltungs- und sozialgerichtlichen Verfahren, in denen der Amtsermittlungsgrundsatz gilt, durch Vertragsschluss zwischen den Parteien möglich. Doch auch dieser Schutz ist nicht umfassend, da an der Mediation beteiligte Dritte nicht von der vertraglich vereinbarten Vertraulichkeit erfasst werden.

Um einen umfassenden Schutz der Vertraulichkeit zu gewährleisten, empfiehlt sich eine gesetzliche Regelung nach dem Vorbild des von der UNCITRAL verabschiedeten „Model Law On International Commercial Conciliation“, nach deren Art. 9 die Vertraulichkeit der in der Mediation erlangen Informationen gefordert und in Art. 10 (1) jedem, also auch jedem Dritten, an der Mediation Beteiligten ein Zeugnisverweigerungsrecht eingeräumt wird. Der Vorentwurf der EUKommission, genauer Art. 6 (1) des Vorentwurfes greift hier zu kurz. Er sieht lediglich die Verschwiegenheit des Mediators sowie seiner Hilfspersonen vor und sichert diese durch ein Beweisverwertungsverbot ab. Dritte werden folglich nicht erfasst.

\section{Kostenaspekte der gerichtsnahen Mediation}

\section{Kosten für Gerichts- und Mediationsverfahren}

Zunächst soll geklärt werden, welche direkten Kosten den Parteien für die gerichtsnahe Mediation im Vergleich zum herkömmlichen streitigen Gerichtsverfahren entstehen.

\section{a) Gerichts- und Mediationskosten nach geltendem Recht}

\section{aa) Zivilprozess}

Im Zivilprozess entstehen gemäß $\int 3$ Abs. 2 GKG in Verbindung mit Nr. 1210 Anlage $1 \mathrm{GKG}$ für das streitige mit einem Urteil abschließende Verfahren drei Gerichtsgebühren. Schließen die Parteien dagegen einen Prozessvergleich, wird gemäß \ 3 Abs. 2 GKG in Verbindung mit Nr. 1211 Abs. 3 Anlage 1 GKG ledig- 
lich eine Gebühr fällig. ${ }^{256}$ Zusätzlich können Kosten für Zeugen, Sachverständige und sonstige gerichtliche Auslagen entstehen.

134 Für die Durchführung der gerichtsnahen Mediation selbst werden derzeit noch keine gesonderten Gebühren erhoben. Das bedeutet, dass, sofern die Parteien die in der Mediation ausgehandelte Vereinbarung als Prozessvergleich protokollieren lassen, wie im herkömmlichen streitigen Verfahren gemäß \ 3 Abs. 2 GKG in Verbindung mit Nr. 1211 Abs. 3 Anlage 1 GKG nur eine Gerichtsgebühr fällig wird. Zusätzliche Kosten etwa für Zeugen oder Sachverständige werden dagegen in der Regel nicht entstehen, so dass für die Zeit der Kostenfreiheit der gerichtsnahen Mediation im Hinblick auf die Gerichtskosten insgesamt von einer Kostenersparnis der Parteien auszugehen ist, wenn sie sich für die Mediation entscheiden und das Mediationsverfahren erfolgreich ist. Scheitern die Mediationsverhandlungen, so entstehen zumindest keine höheren Kosten als im Falle eines herkömmlichen streitigen Gerichtsverfahrens.

\section{bb) Verwaltungsgerichtsverfahren}

135 Ebenso wie im Zivilprozess entsteht im verwaltungsgerichtlichen Verfahren gemäß \ 3 Abs. 2 GKG in Verbindung mit Nr. 5110 Anlage 1 GKG für das Verfahren selbst eine Gerichtsgebühr in Höhe einer dreifachen Gebühr, die sich gemäß Nr. 5111 Anlage 1 GKG bei einer Beendigung des Verfahrens durch Klagerücknahme, durch Anerkenntnis- oder Verzichtsurteil, durch gerichtlichen Vergleich oder durch Erledigungserklärungen gemäß 161 Abs. 2 VwGO, sofern keine Entscheidung über die Kosten ergeht oder die Entscheidung einer zuvor mitgeteilten Einigung der Beteiligten über die Kostentragung oder der Kostenübernahmeerklärung eines Beteiligten folgt, jedoch auf eine einfache Gebühr ermäßigt. Insoweit hat durch die Neufassung des Gerichtskostengesetzes (GKG) in Art. 1 des am 1. Juli 2004 in Kraft getretenen Kostenrechtsmodernisierungsgesetzes eine Harmonisierung der Gerichtskosten der verschiedenen Gerichtsbarkeiten stattgefunden. Bei Durchführung eines Mediationsverfahrens fällt, sofern sich die Konfliktparteien in der Mediation einigen und das gerichtliche Verfahren in einer der genannten Weisen beendet wird, damit regelmäßig eine einfache Gerichtsgebühr an.

256 Gleiches gilt, wenn die Klage zurückgenommen wird, sowie im Fall einer beiderseitigen Erledigungserklärung gemäß \91a ZPO, soweit keine Entscheidung über die Kosten ergeht oder die Kostenentscheidung einer zuvor mitgeteilten Einigung der Parteien über die Kostentragung oder der Kostenübernahmeerklärung einer Partei entspricht. 


\section{cc) Sozialgerichtsverfahren}

Das sozialgerichtliche Verfahren ist für Versicherte, Leistungsempfänger einschließlich Hinberbliebenenleistungsempfänger, Behinderte oder deren Sonderrechtsnachfolger nach \56 SGB I gemäß \183 S. 1 SGG kostenfrei. Damit ist sowohl das herkömmliche sozialgerichtliche Verfahren als auch die Teilnahme an einer gerichtsnahen Mediation kostenfrei. Im Hinblick auf eine zukünftige Kostenregelung für die gerichtsnahe Mediation müsste mit Rücksicht auf das Sozialstaatsprinzip für die nach $\int 183 \mathrm{~S}$. 1 SGG privilegierten Beteiligten die Inanspruchnahme des gerichtlichen Mediationsangebotes auch in Zukunft kostenfrei bleiben. Wettbewerbsrechtliche Bedenken bestehen insoweit nicht, weil der Staat damit nur seiner verfassungsrechtlich verankerten Sozialverantwortung gerecht wird.

Für die andere, nicht zu dem in \183 S. 1 SGG genannten Personenkreis gehörende Partei, fällt gemäß $\int 184$ Abs. 1 S. 1, Abs. 2 SGG eine Pauschalgebühr in Höhe von 150 bis 300 Euro an. Nicht gemäß \ 183 S. 1 SGG privilegiert sind insbesondere Körperschaften und Anstalten des öffentlichen Rechts, kassenärztliche und kassenzahnärztliche Vereinigungen, kommunale Gebietskörperschaften, Familienausgleichskassen, Innungen und Innungsverbände, Landwirtschaftskammern, Ärztekammern, aber auch Ärzte, Zahnärzte, Psychotherapeuten und andere Personen, soweit sie nicht in ihrer Eigenschaft als Versicherte oder sonstige Leistungsberechtigte beteiligt sind. ${ }^{257}$

In den Fällen, in denen weder der Kläger noch der Beklagte zu den gemäß $\int 183$ SGG privilegierten Personen gehört, werden gemäß \ 197 a Abs. 1 S. 1 SGG auch im sozialgerichtlichen Verfahren Kosten nach dem GKG erhoben. Gemäß \ 3 Abs. 2 GKG in Verbindung mit Nr. 7110 Anlage 1 GKG entsteht dann ebenso wie im zivil- und verwaltungsgerichtlichen Verfahren für das Verfahren selbst eine Gerichtsgebühr in Höhe einer dreifachen Gebühr, die sich gemäß Nr. 7111 Anlage 1 GKG bei einer Beendigung des Verfahrens durch Klagerücknahme, durch Anerkenntnisurteil, durch gerichtlichen Vergleich oder durch Erledigungserklärungen gemäß \ 197 a Abs. 1 S. 1 SGG in Verbindung mit \ 161 Abs. 2 VwGO, sofern keine Entscheidung über die Kosten ergeht oder die Entscheidung einer zuvor mitgeteilten Einigung der Beteiligten über die Kostentragung oder der Kostenübernahmeerklärung eines Beteiligten folgt, auf eine einfache Gebühr ermäßigt.

${ }^{257}$ Meyer-Ladewig, SGG, \184 Rn. 3. 


\section{b) Rechtsanwaltskosten}

\section{aa) Gebühren bei gerichtsnaher Mediation im Zivilprozess}

139 Das mit Inkrafttreten des Kostenrechtsmodernisierungsgesetzes am 7. Juli 2004 neu eingeführte Rechtsanwaltsvergütungsgesetz (RVG), welches die bis dahin geltende Bundesrechtsanwaltsgebührenordnung (BRAGO) ersetzt hat, hat für die Berechnung der anwaltlichen Gebühren in Zusammenhang mit der gerichtsnahen Mediation wichtige Änderungen gebracht. Ein Ziel des neuen RVG ist es, die außergerichtliche einvernehmliche Streitbeilegung dadurch zu fördern, dass der Bedeutung der außergerichtlichen Tätigkeit des Anwalts durch entsprechende Gebührenanreize Rechnung getragen wird. ${ }^{258}$ Dementsprechend kann der Rechtsanwalt für seine Tätigkeit in der gerichtsnahen Mediation nun nicht nur die in Zusammenhang mit der Einleitung des streitigen Gerichtsverfahrens entstehenden Gebühren sowie im Falle einer Einigung der Parteien in der Mediation auch eine Vergleichs- bzw. Einigungsgebühr berechnen. Es steht ihm - anders als nach der bisherigen Rechtslage nach der BRAGO - auch ein gesetzlicher Gebührentatbestand für seine begleitend beratende Tätigkeit im Mediationsverfahren zur Verfügung. Nach der BRAGO war es dagegen nicht möglich, für das außergerichtliche Tätigwerden eine gesetzliche Gebühr zu berechnen, wenn ein Rechtsstreit in derselben Sache bereits anhängig war. Denn gemäß \ 37 Nr. 2 BRAGO gehörten außergerichtliche Verhandlungen, wenn ein gerichtliches Verfahren anhängig war, zum Rechtszug und wurden bereits mit der Prozessgebühr gemäß $\ 31$ Abs. $1 \mathrm{Nr}$. 1 BRAGO abgegolten. ${ }^{259}$ Der Rechtsanwalt stand damit, sofern er nicht auf eine Honorarvereinbarung hinwirkte, im Falle einer - erfolgreich durchgeführten gerichtsnahen Mediation finanziell schlechter da als bei einem herkömmlichen Gerichtsverfahren mit der Möglichkeit, neben der Prozessgebühr gemäß \31 Abs. 1 Nr. 1 BRAGO und gegebenenfalls einer Vergleichsgebühr gemäß $\$ 23$ Abs. 1 S. 3 BRAGO auch die Verhandlungs- und die Beweisgebühr gemäß $\ 31$ Abs. 1 Nr. 2 und 3 BRAGO zu verdienen. ${ }^{260}$ Nur wenn weitere, nicht zum Streitgegenstand des anhängigen Gerichtsverfahrens gehörende Ansprüche in die Mediationsverhandlungen einbezogen wurden, konnte der Anwalt neben den Gebühren der \\31 ff. BRAGO nach dem Wert der anhängigen Ansprüche für sein außerge-

\footnotetext{
258 BT-Drucks. 15/1971, S. 2.

259 Siehe dazu Enders, JurBüro 1998, 337, 338.

$260 \mathrm{Um}$ diesen Gebührenverlust durch die gerichtsnahe Mediation zu vermeiden wurde an den Gerichten teilweise dazu übergegangen, die Sache vor der gerichtlichen Protokollierung eines in der Mediation geschlossenen Vergleichs noch einmal zu erörtern, um zumindest die Erörterungsgebühr gemäß \31 Abs. 1 Nr. 4 BRAGO entstehen zu lassen. Im Hinblick auf den Grundsatz der Vertraulichkeit der Mediationsverhandlung war diese Vorgehensweise jedoch zweifelhaft.
} 
richtliches Tätigwerden auch die Gebühren des \118 BRAGO nach dem Wert der nicht anhängigen Ansprüche berechnen. ${ }^{261}$

Nach dem RVG stehen dem Rechtsanwalt, der seinen Mandanten in einer gerichtsnahen Mediation begleitet, dagegen die folgenden Gebühren zu:

In Zusammenhang mit der Einleitung des zivilgerichtlichen Verfahrens kann der Rechtsanwalt gemäß Nr. 3100 VV RVG eine Verfahrensgebühr berechnen. Die Verfahrensgebühr entspricht im Wesentlichen der Prozessgebühr gemäß \31 Abs. 1 Nr. 1 BRAGO, ${ }^{262}$ wurde jedoch im Vergleich zu der einfachen Prozessgebühr auf 1,3 erhöht. Eine Terminsgebühr gemäß Nr. 3104 VV RVG, welche die Verhandlungs- und die Erörterungsgebühr ersetzt hat und im Vergleich zu den genannten einfachen Gebühren nach der BRAGO auf 1,2 erhöht wurde, kann der Rechtsanwalt für seine Tätigkeit im streitigen Gerichtsverfahren dagegen ebenso wenig wie nach bisherigem Recht berechnen, wenn die Mediation noch vor der mündlichen Verhandlung eingeleitet wird. ${ }^{263}$

Anders als nach der bisherigen Rechtslage kann der Rechtsanwalt die Terminsgebühr gemäß Nr. 3104 VV RVG mit einem Gebührensatz von 1,2 aber durch seine begleitend beratende Tätigkeit in der gerichtsnahen Mediation verdienen. Denn Abs. 3 der Vorbemerkung zu Teil 3 VV RVG bestimmt, dass der Rechtsanwalt die Terminsgebühr nicht nur für die Wahrnehmung gerichtlicher Termine, sondern auch für die Mitwirkung an auf die Vermeidung oder Erledigung des Verfahrens gerichteten Besprechungen ohne Beteiligung des Gerichts erhält. Nichts anderes ist bei der gerichtsnahen Mediation der Fall, so dass nunmehr die außergerichtliche Tätigkeit des Anwalts parallel zu einem anhängigen Gerichtsverfahren der Tätigkeit im streitigen Gerichtsverfahren gebührenrechtlich gleichgestellt ist.

Im Fall einer Einigung der Parteien im Rahmen der gerichtsnahen Mediation kann der Anwalt zudem eine Einigungsgebühr gemäß Nr. 1003 VV RVG in Höhe einer einfachen Gebühr berechnen. ${ }^{264}$ Die Einigungsgebühr nach Nr. 1003 VV RVG hat die zuvor geltenden Vergleichsgebühr gemäß \ 23 Abs. 1 S. 3 BRAGO ersetzt.

261 Es handelte sich dann gebührenrechtlich im zwei verschiedene Angelegenheiten, siehe dazu Enders, JurBüro 1998, 337, 338.

262 Braun, Gebührenabrechnung nach dem neuen Rechtsanwaltsvergütungsgesetz, S. 65; Müller-Rabe, in: Gerold/Schmidt/v. Eicken/Madert/Müller-Rabe, RVG, VV Vorb. 3 Rn. 25.

263 Eine Beweisgebühr wird vom RVG gar nicht erst vorgesehen.

${ }^{264} \mathrm{Da}$ bei der gerichtsnahen Mediation regelmäßig ein gerichtliches Verfahren anhängig ist, beträgt die Einigungsgebühr anders als im außergerichtlichen Bereich nicht 1,5 gemäß Nr. $1000 \mathrm{VV}$ RVG. 
Der Höhe nach hat sich an der Gebühr durch die Einführung des RVG nichts geändert.

144 Schließlich ist der Fall, dass bei außergerichtlichen Verhandlungen gemäß Nr. 3104 VV RVG auch Verhandlungen über nicht zum Streitgegenstand des anhängigen Gerichtsverfahrens gehörende Ansprüche geführt werden, nunmehr ausdrücklich geregelt: Gemäß Abs. 2 der Anmerkung zu Nr. 3104 VV RVG entsteht die volle Terminsgebühr in Höhe von 1,2 auch aus dem Wert der nicht rechtshängigen Ansprüche. ${ }^{265}$ Nur wenn der Anwalt bereits aus einem anderen Verfahren Gebühren wegen dieser Ansprüche erhält, hat eine Anrechnung zu erfolgen. ${ }^{266}$

145 Als Ergebnis ist festzuhalten, dass der Anwalt im Hinblick auf die gesetzlichen Gebühren nach dem RVG nunmehr keine Gebührenverluste im Vergleich zu seiner Tätigkeit in einem herkömmlichen Gerichtsverfahren befürchten muss, wenn er seinem Mandanten die Inanspruchnahme des gerichtlichen Mediationsangebotes empfiehlt und die Partei in der Mediation begleitend berät. ${ }^{267}$

\section{bb) Gebühren bei gerichtsnaher Mediation im verwaltungs- gerichtlichen Verfahren}

146 Das Kostenrechtsmodernisierungsgesetz hat im Bereich der anwaltlichen Gebühren für eine Harmonisierung gesorgt. Die relevanten Gebührentatbestände in Teil 1 und Teil 3 des Vergütungsverzeichnisses zum RVG gelten für Verfahren der öffentlich-rechtlichen Gerichtsbarkeiten ebenso wie für bürgerliche Rechtsstreitigkeiten. Im Hinblick auf die Gebühren, die der Rechtsanwalt für seine Tätigkeit im Zusammenhang mit der gerichtsnahen Mediation beim Verwaltungsgericht berechnen kann, wird daher auf die vorstehenden Ausführungen zum Zivilprozess verwiesen.

147 Der Umstand, dass im Rahmen des niedersächsischen Modellprojekts zur gerichtsnahen Mediation im Bereich der öffentlich-rechtlichen Gerichtsbarkeiten regelmäßig auf die Protokollierung eines Prozessvergleichs und damit auf einen Vollstreckungstitel verzichtet wird, hat auf die Höhe der anwaltlichen Gebühren keine Auswirkung. Denn die Einigungsgebühr entsteht - unabhängig von der

\footnotetext{
265 Schneider/Mock, Das neue Gebührenrecht für Anwälte, S. 159.

266 Römermann, in: Hartung/Römermann, RVG, VV Teil 3 Rn. 65; Schneider/Mock, Das neue Gebührenrecht für Anwälte, S. $159 \mathrm{f}$.

267 Näher zum Honoraranspruch der begleitend beratenden Anwälte in der gerichtsnahen Mediation Volkmann, Mediation im Zivilprozess. Rechtliche Rahmenbedingungen für ein gerichtsinternes Mediationsangebot, S. 141 ff.
} 
Protokollierung eines Prozessvergleichs - mit der Einigung der Konfliktparteien im Laufe der Mediationsverhandlungen.

\section{cc) Gebühren bei gerichtsnaher Mediation im sozialgerichtlichen Verfahren}

Für seine Tätigkeit im sozialgerichtlichen Verfahren kann der Rechtsanwalt dagegen in den Fällen, in denen das GKG nicht anwendbar ist ( $\int 183$ SGG), gemäß \ 3 Abs. 1 S. 1 RVG nur Betragsrahmengebühren berechnen. Die einzelnen dem Rechtsanwalt zustehenden Betragsrahmengebühren ergeben sich im Gegensatz zu der bisherigen Regelung in der BRAGO nunmehr aus dem Vergütungsverzeichnis des RVG. ${ }^{268}$ Neben einer Verfahrensgebühr gemäß Nr. 3102 VV RVG in Höhe von 40,- bis 460,- Euro sieht das RVG nun auch eine Terminsgebühr gemäß Nr. 3106 VV RVG in Höhe von 20,- bis 380,- Euro vor, für die Abs. 3 der Vorbemerkung zu Teil 3 VV RVG ebenso gilt wie für die Terminsgebühr im zivil- und verwaltungsgerichtlichen Verfahren. Insoweit kann auf die vorstehenden Ausführungen zum Zivilprozess verwiesen werden. Mit dem RVG wurde zudem auch für den Bereich der sozialrechtlichen Angelegenheiten eine besondere Vergleichsbzw. Erledigungsgebühr neu eingeführt: ${ }^{269}$ Die Rahmengebühr in Höhe von 40,bis 520,- Euro entsteht nun gemäß Nr. 1005 VV RVG auch im Fall einer Einigung oder Erledigung in sozialrechtlichen Angelegenheiten, in denen im gerichtlichen Verfahren Betragsrahmengebühren entstehen, wobei sie sich gemäß Nr. 1006 VV RVG jedoch auf 30,- bis 350,- Euro reduziert, wenn über den Gegenstand ein gerichtliches Verfahren anhängig ist. Damit wurde für die Anwaltschaft auch ein positiver Anreiz gesetzt, auch in sozialrechtlichen Angelegenheiten an einem einvernehmlichen Konfliktbeilegungsverfahren teilzunehmen.

In sonstigen Verfahren vor den Sozialgerichten, sofern der Auftraggeber nicht zu den in \183 SGG genannten Personen gehört, werden die Gebühren - wie im zivil- und verwaltungsgerichtlichen Verfahren auch - gemäß $\iint 3$ Abs. 1 S. 2, 2 Abs. 1 RVG nach dem Wert berechnet, den der Gegenstand der anwaltlichen Tätigkeit hat. Insoweit wird auf die Ausführungen zum Zivilprozess verwiesen.

\footnotetext{
268 Dinkat, in: Mayer/Kroiß, RVG, \ 3 Rn. 11.

${ }^{269}$ Dinkat, in: Mayer/Kroiß, RVG, \ 3 Rn. 14.
} 


\section{Erstattung der Kosten für Gerichts- und Mediationsverfahren}

\section{a) Erstattung durch den Gegner}

\section{aa) Zivilprozess}

\section{(1) Scheitern der Mediationsverhandlungen und Fortführung des gerichtlichen Verfahrens}

150 Grundsätzlich gilt für den deutschen Zivilprozess das Prinzip der Kostenerstattung: Scheitern die Mediationsverhandlungen und wird der Rechtsstreit durch Urteil entschieden, so hat die unterliegende Partei die Kosten des Rechtsstreits zu tragen, insbesondere die dem Gegner erwachsenen, zur zweckentsprechenden Rechtsverfolgung oder Rechtsverteidigung notwendigen Kosten zu erstatten (vgl. $\left.\int 91 \mathrm{ZPO}\right)$.

151 Zu einer zweckentsprechenden Rechtsverfolgung oder Rechtsverteidigung notwendig sind Kosten, wenn die ihnen zugrunde liegende Handlung zu einer sachdienlichen Rechtsverfolgung oder Rechtsverteidigung erforderlich und geeignet war, ${ }^{270}$ wobei auf den Zeitpunkt, zu welchem die Tätigkeit vorzunehmen war, abzustellen ist. ${ }^{271}$ Dazu zählen die Kosten für die anwaltliche Begleitung in der Mediation ebenso wie die zukünftig für das gerichtliche Mediationsangebot zu erhebenden Gerichtskosten: 272

152 Im Hinblick auf die Kosten für die anwaltliche Begleitung in der Mediation ergibt sich dies aus Abs. 3 der Vorbemerkung zu Teil 3 VV RVG, der auch für die Tätigkeit des Anwalts in außergerichtlichen Verhandlungen, wenn ein Rechtsstreit in derselben Sache anhängig ist, nunmehr einen gesetzlichen Gebührentatbestand geschaffen hat. Nach der alten Rechtslage infolge Honorarvereinbarung entstehende Anwaltkosten waren nicht erstattungsfähig, da es sich hierbei nicht um gesetzliche Gebühren des Rechtsanwalts im Sinne von \91 Abs. 2 ZPO handelte. Bei der durch die anwaltliche Begleitung in der gerichtsnahen Mediation entstehenden Terminsgebühr handelt es sich dagegen um eine gesetzliche Gebühr, die der obsiegenden Partei stets gemäß \91 Abs. 2 ZPO zu erstatten ist.

\footnotetext{
270 Belz, in: MünchKommZPO, Band 1, \ 91 Rn. 17; P. Hartmann, in: Baumbach/Lauterbach/Albers/P. Hartmann, ZPO, \ 91 Rn. 29; Wolst, in: Musielak, ZPO, \ 91 Rn. 8.

271 Belz, in: MünchKomm ZPO, Band 1, \ 91 Rn. 17; Bork, in: Stein/Jonas, ZPO, Band 2, \ 91 Rn. 45.

272 Zum folgenden siehe auch Volkmann, Mediation im Zivilprozess. Rechtliche Rahmenbedingungen für ein gerichtsinternes Mediationsangebot, S. 150 f.
} 
Aus der Wertung des $₫ 278$ Abs. 5 S. 2 ZPO ergibt sich, dass auch die in der Zukunft zu erhebenden Kosten für die Inanspruchnahme des gerichtlichen Mediationsangebots als notwendige und damit erstattungsfähige Kosten des Rechtsstreits anzusehen sind. Für die Erstattung außergerichtlicher Kosten in einem obligatorischen Schlichtungsverfahren im Sinne von \15 a EGZPO wurde bereits entschieden, dass im Falle des Scheiterns der Schlichtung die für das Schlichtungsverfahren aufzuwendenden Kosten als notwendige Vorbereitungskosten im Rahmen der Kostenerstattung nach \91 ZPO in einem nachfolgenden Klageverfahren grundsätzlich erstattungsfähig sind. ${ }^{273}$ Für eine gemäß \ 278 Abs. 5 S. 2 ZPO durchgeführte außergerichtliche Streitbeilegung kann unabhängig davon, ob die Parteien ein gerichtliches oder ein außergerichtliches Konfliktlösungsangebot in Anspruch nehmen, nichts anderes gelten. Zwar erfolgt der Versuch einer einvernehmlichen Streitbeilegung außerhalb des streitigen Gerichtsverfahrens im Fall des $\int 278$ Abs. 5 S. 2 ZPO anders als bei $\ 15$ a EGZPO ausschließlich auf freiwilliger Basis, doch wird man nicht umhin können, auch die Kosten eines Konfliktlösungsversuchs nach \278 Abs. 5 S. 2 ZPO als notwendige Kosten im Sinne von $\int 91$ ZPO anzuerkennen, wenn die Gerichte von der Möglichkeit des \278 Abs. 5 S. 2 ZPO als Mittel zur Förderung der einvernehmlichen außergerichtlichen Streitbeilegung Gebrauch machen und den Parteien den Versuch einer einvernehmlichen Streitbeilegung außerhalb des streitigen Gerichtsverfahrens nahe legen.

Zur Anreizfunktion des $\int 91$ ZPO, insbesondere zur Frage, ob bereits de lege lata Kostensanktionen im Rahmen des \91 ZPO möglich sind, s. unten Rn. 294.

\section{(2) Einigung im Laufe der Mediationsverhandlungen und Protokol- lierung eines Prozessvergleichs}

Wird hingegen bei erfolgreich verlaufenen Mediationsverhandlungen die Mediationsvereinbarung als Prozessvergleich gemäß \ 794 Abs. 1 Nr. 1 ZPO protokolliert, so sind gemäß \98 ZPO die Kosten des abgeschlossenen Vergleichs als gegeneinander aufgehoben anzusehen, wenn nicht die Parteien ein anderes vereinbart haben. Dabei ist eine vertragliche Vereinbarung über die Verteilung der Kostenlast der Regelfall. Wie im Bereich der außergerichtlichen Mediation auch, wird im Fall einer Einigung der Parteien in der Regel davon auszugehen sein, dass sie sich die Kosten des Verfahrens teilen. ${ }^{274}$ Aber auch für den Fall des Scheiterns der Mediationsverhandlungen kann die Verteilung der Kostenlast vertraglich gere-

\footnotetext{
273 BayObLG ZKM 2004, 281 m. Anm. Wagner ZKM 2004, 282 ff.

${ }^{274}$ Monßen, ZKM 2003, 116, 118; Wegmann, Zeitschrift für Mediation 1999, 80, 82.
} 
gelt werden, z.B. nach dem Vorbild der sog. Michigan-Mediation: Hier werden auch der obsiegenden Partei die Kosten des gesamten Rechtsstreits auferlegt, wenn sie zuvor einen Einigungsvorschlag der anderen Partei unbegründet abgelehnt hat. ${ }^{275}$

\section{(3) Immaterielle Kosten}

156 Für die Kostenerstattungspflicht des $\int 91$ ZPO gilt ein materieller Kostenbegriff. Immaterielle Kosten wie z.B. die psychische Belastung eines Beklagten, der zu Unrecht in einen Rechtsstreit verwickelt wird, werden selbst bei Obsiegen im Prozess nicht ersetzt.

\section{bb) Verwaltungsgerichtsverfahren}

157 Für den Verwaltungsprozess ergibt sich hier grundsätzlich nichts anderes als für den Zivilprozess. Gemäß $\int 154$ Abs. 1 VwGO sind die Kosten des Verfahrens vom unterliegenden Teil zu tragen. Bei teilweisem Obsiegen und Unterliegen, sind die Kosten gemäß \ 155 Abs. 1 S. 1 VwGO gegeneinander aufzuheben oder verhältnismäßig zu teilen. Wird ein Vergleich geschlossen und haben die Parteien keine Vereinbarung über die Kosten getroffen, so fallen gemäß \ 160 S. 1 VwGO die Gerichtskosten jedem Teil zur Hälfte zur Last, während gemäß \ 160 S. 2 VwGO die außergerichtlichen Kosten von jedem Beteiligten selbst zu tragen sind. Wie im Zivilprozess ist aber auch im verwaltungsgerichtlichen Verfahren in aller Regel davon auszugehen, dass im Fall einer Einigung in der Mediation auch eine Vereinbarung zur Verteilung der Kostenlast getroffen wird. Insoweit kann auf die vorstehenden Ausführungen verwiesen werden.

\section{cc) Sozialgerichtsverfahren}

158 Die außergerichtlichen Kosten der in \183 SGG privilegierten Personen können gemäß \193 Abs. 1 SGG vom Gegner zu erstatten sein. ${ }^{276}$ Die erstattungsfähigen außergerichtlichen Kosten umfassen gemäß \ 193 Abs. 3 SGG insbesondere die gesetzlichen Rechtsanwaltsgebühren. Nicht erstattungsfähig sind dagegen gemäß \ 193 Abs. 4 S. 1 die Aufwendungen der Behörden der in \ 184 Abs. 1 SGG genannten Gebührenpflichtigen. Die Kostenentscheidung gemäß \193 SGG erfolgt nach billigem Ermessen und ist nicht an den Ausgang des Verfahrens gebun-

\footnotetext{
275 Zur Michigan-Mediation siehe auch Risse/C. Wagner, in: Haft/v. Schlieffen, Handbuch Mediation, S. 987, 1020; Eidenmüller, in: Duve/Eidenmüller/Hacke, Mediation in der Wirtschaft, S. 240 f. 276 Meyer-Ladewig, SGG, \193 Rn. 4.
} 
den. ${ }^{277}$ Gleichwohl hat das Gericht bei seiner Ermessensentscheidung das Ergebnis des Rechtsstreits und den Sach- und Streitstand zu berücksichtigen und sich am Rechtsgedanken der $\iint 91 \mathrm{ff}$. ZPO zu orientieren. ${ }^{278}$ Deshalb ergibt sich unter Berücksichtigung der vorstehend genannten Besonderheiten - für die Kostenverteilung grundsätzlich nichts anderes als im Zivil- und Verwaltungsprozess.

Für Fälle, in denen weder der Kläger noch der Beklagte zu den in \183 SGG genannten Personen gehört, verweist \ 197 a Abs. 1 SGG auf die \$S 154 bis 162 VwGO, so dass insoweit grundsätzlich nichts anderes gilt als im verwaltungsgerichtlichen Verfahren.

\section{b) Kostenerstattung durch die Rechtsschutzversicherung}

Hat eine Partei eine Rechtsschutzversicherung abgeschlossen, so werden die Kosten des Rechtsstreits von der Rechtsschutzversicherung erstattet. Unproblematisch besteht ein Erstattungsanspruch für die Kosten und Kostenvorschüsse, die zur Wahrnehmung der rechtlichen Interessen notwendig sind. Das sind die Kosten des Anwaltes nach der gesetzlichen Gebührenordnung, die Gerichtskosten einschließlich der Zeugengelder und Sachverständigengebühren sowie die Vollstreckungskosten, außerdem die Kosten des Prozessgegners, soweit der Versicherte diese Kosten zu tragen hat. Damit ermöglicht die Rechtsschutzversicherung also den Parteien für die versicherten Kosten - bei Fehlen eines Selbstbehaltes ein (privat) grenzkostenfreies Prozessieren, also den einzelnen Prozess ohne zusätzliche eigenen (monetären) Kostenaufwendungen durchzuführen. ${ }^{279}$

Im Fall der gerichtsnahen Mediation ist folglich davon auszugehen, dass die in Zusammenhang mit dem gerichtlichen Verfahren entstehenden Kosten unproblematisch von der Rechtsschutzversicherung getragen werden. Anders als nach bisheriger Rechtslage gehört seit Inkrafttreten des RVG dazu auch die durch die anwaltliche Begleitung in der Mediation entstehende Terminsgebühr gemäß Nr. 3104 VV RVG. ${ }^{280}$ Fraglich ist jedoch, ob auch von einer Übernahme der in Zukunft möglicherweise erhobenen gerichtlichen Mediationskosten durch die Rechtsschutzversicherungen auszugehen ist.

\footnotetext{
277 Meyer-Ladewig, SGG, \ 193 Rn. 12.

278 Meyer-Ladewig, SGG, \ 193 Rn. 12.

279 Adams, Ökonomische Analyse des Zivilprozesses, 1981, S. 114.

280 Nach der BRAGO konnte der Rechtsanwalt dagegen keine gesetzlichen Gebühren für seine begleitend beratende Tätigkeit in der Mediation berechnen und war auf den Abschluss einer Honorarvereinbarung angewiesen, deren Kosten nicht ohne weiteres von den Rechtsschutzversicherungen übernommen worden wären, siehe dazu Spindler/Apel/Spalckhaver, ZKM 2003, 192, 195.
} 
162 In der Rechtspraxis ergibt sich ein uneinheitliches Bild für die Übernahme der Kosten eines Mediationsverfahrens. Einzelne Rechtsschutzversicherer übernehmen die Kosten für die Mediation unter den Voraussetzungen, dass der Mediator Rechtsanwalt ist, das Verfahren den rechtlichen Interessen des Versicherten dient und nicht nur "psychologisch-sozial“ motiviert ist und außerdem die Mediation ein Rechtsgebiet betrifft, das die Police abdeckt, so dass im Familien- und Erbrecht eine Kostenübernahmen von vornherein ausscheidet. Im Bereich der außergerichtlichen Mediation erklärten sich 2004 gleichwohl etwa ein Drittel der Rechtsschutzversicherungen nicht bereit, die Kosten für die Inanspruchnahme eines Mediators zu übernehmen.281

163 Auf eigene Anfrage der Universität Göttingen gab der Gesamtverband der Deutschen Versicherungswirtschaft e.V. (GDV) an, dass Mediationskosten grundsätzlich nicht vom Rechtsschutzversicherungsschutz erfasst werden. Übernehmen einzelne Versicherungsanbieter dennoch diese Kosten, handele es sich dabei um Leistungen aus Kulanz. Der GDV sieht als übernahmefähig vielmehr nur Kosten für einen Anwalt an, der Interessenvertreter des Versicherungsnehmers ist. Nach Ansicht des GDV fallen daher Kosten für ein Mediationsverfahren nicht unter $₫ 5$ Abs. 1 d ARB 94/2000, wonach der Rechtsschutzversicherer u.a. auch die Gebühren eines Schieds- und Schlichtungsverfahrens bis zur Höhe der Gebühren trägt, die im Falle der Anrufung eines zuständigen staatlichen Gerichts erster Instanz entstehen. Nach einer historischen Auslegung des $₫ 5$ Abs. 1 d ARB 94/2000 wird das Mediationsverfahren hiervon nicht erfasst, auch widersprechen - wie bereits erwähnt - Sinn und Zweck der Rechtsschutzversicherung (Interessenvertretung des Versicherungsnehmers, vgl. \1 Abs. 1 d ARB 94/2000) einer Kostenübernahme. ${ }^{282}$ Eine Erweiterung des Versicherungsschutzes auch auf die Inanspruchnahme von Mediation zur Konfliktlösung ist zu Beginn des Jahres 2005 daher noch nicht abzusehen. Vielmehr bleibt hier abzuwarten, ob (und vor allem wann) die Versicherungen die Kosten für eine gerichtlich angeregte und im Gericht durchgeführte Mediation in der Zukunft bereitwilliger übernehmen.

281 Anfrage von FINANZtest 2004, 14, jedoch ohne weitere über die genannten Voraussetzungen hinausgehende Angaben.

282 Anders Bauer, in Harbauer, RSV, $\ 5$ ARB 94/2000, Rn. 8 nach dem dann die Kosten nach $₫ 5$ Abs. 1 d ARB 94/2000 übernommen werden können, wenn ein uneingeschränkter Rechtsschutz besteht. 


\section{Prozess- und Mediationskostenhilfe}

\section{a) Zivilprozess}

Fraglich ist, ob eine Prozesskostenhilfeberechtigung sich auch auf etwaige Kosten im Zusammenhang mit gerichtsnaher Mediation erstrecken kann. Die Prozesskos-

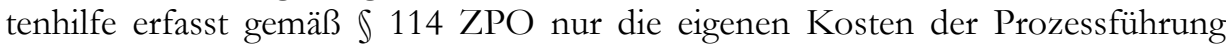
(Anwaltskosten ( $₫ 1$ Abs. 1 RVG), gerichtliche Gebühren und Auslagen ( $₫ 1$ Abs. 1 GKG)).283 Anders als nach der bisherigen Rechtslage werden bei der gerichtsnahen Mediation nun nicht nur die in Zusammenhang mit der Einleitung des gerichtlichen Verfahrens entstehenden Gerichts- und Anwaltskosten, sondern wegen der Einführung des Gebührentatbestands der Nr. 3104 VV RVG - auch die Kosten für eine anwaltliche Begleitung in der Mediation von der Prozesskostenhilfe erfasst, so dass bedürftigen Parteien die Teilnahme an einer Mediation unter anwaltlicher Begleitung nicht länger verschlossen bleibt. ${ }^{284}$

Im Hinblick auf die in Zukunft möglicherweise erhobenen gerichtlichen Mediationskosten ist die Einführung einer der Prozesskostenhilfe entsprechenden Mediationskostenhilfe geboten. So kann der Staat wirkungsvoll Anreize setzen, auch tatsächlich die für ihn kostengünstigere Alternative der gerichtsnahen Mediation zu wählen. Auch muss der Staat, wenn er den Parteien die Wahrnehmung des gerichtlichen Mediationsangebotes als Alternative zu einem gerichtlichen Verfahren, für welches Prozesskostenhilfe gewährt wird, nahe legt, dafür sorgen, dass bedürftigen Parteien der Weg der gerichtsnahen Mediation nicht aus Kostengründen verschlossen bleibt. Dies ergibt sich aus seiner Sozialverantwortung: Die Sicherung eines gleichwertigen Rechtsschutzes ist ein sozialstaatliches Gebot im Sinne von Art. 20 Abs. 1 GG. ${ }^{285}$

\section{b) Verwaltungs- und sozialgerichtliches Verfahren}

Gemäß $₫ 166$ VwGO und $\ 73$ a Abs. 1 S. 1 SGG gelten die Vorschriften der Zivilprozessordnung über die Prozesskostenhilfe im verwaltungs- und sozialgerichtlichen Verfahren entsprechend. Insoweit kann auf die Ausführungen zum Zivilprozess verwiesen werden.

\footnotetext{
283 Philippi, in Zöller, ZPO, \ 114 Rn. 15.

284 Volkmann, Mediation im Zivilprozess. Rechtliche Rahmenbedingungen für ein gerichtsinternes Mediationsangebot, S. 155 ff., auch zum folgenden.

285 BVerfGE 9, 124, 131 f.; Sachs, in: Sachs, GG, Art. 20 Rn. 48.
} 


\section{Zweiter Teil: Rechtsökonomische Analyse}

\section{I. Überblick}

167 Damit die gerichtsnahe Mediation tatsächlich eine Alternative zum herkömmlichen streitigen Gerichtsverfahren darstellen kann, bedarf es der Akzeptanz der Beteiligten. Im folgenden soll untersucht werden, ob unter den gegenwärtigen rechtlichen Rahmenbedingungen für alle Beteiligten Anreize gegeben sind, gerichtsnahe Mediation als Alternative zum streitigen Verfahren zu wählen bzw. welche Anreize gegebenenfalls gesetzt und welche Rahmenbedingungen geändert werden müssen, damit die Beteiligten tatsächlich in der gewünschten Weise handeln. Wünschenswert, aber außerhalb des Forschungsauftrages liegend, wäre dabei die Erstreckung dieses Ansatzes auf die vorgerichtliche Mediation und deren Anreizstrukturen, um ein einheitliches Bild der Chancen einer Mediation zur Entlastung der Justiz zu gewinnen.

\section{Einleitung}

\section{Methodischer Ansatz}

168 Ob und unter welchen Rahmenbedingungen diese Akzeptanz der Beteiligten vorliegt und wie gegebenenfalls Faktoren des Mediationseinsatzes geändert werden müssen, wurde durch eine empirisch gestützte Begleitforschung untersucht, die sich auf einen verhaltensbezogenen Forschungsansatz stützt, der die Brücke zwischen Empirie und normativen Aussagen schlagen kann. So können gleichzeitig wichtige Fundamente für die weitere Ausformung des Mediationsprozesses und seine Einbettung in die Prozessrechtsordnungen gelegt werden.

169 Ausgangspunkt dieser Begleitforschung ist ein ökonomischer Ansatz, der nach einer effizienten Verteilung der Anreiz- und Kostenstrukturen für die Beilegung von Konflikten fragt. Der methodische und theoretische Hintergrund für diesen Ansatz sind die Neue Institutionenökonomik und die Ökonomische Analyse des Rechts, die rechtliche Rahmenbedingungen auf ihre Auswirkungen und ihre effiziente Ausgestaltung zur Erreichung einer optimalen Transaktionskostenstruktur zwischen Parteien, aber auch zwischen staatlichen Institutionen und Bürgern (Verwaltungsverfahren, Sozialgericht) untersucht. 
Die Neue Institutionenökonomik beschäftigt sich mit der Frage, welche Regelsysteme geeignet sind, erwünschte Interaktionen zu ermöglichen und unerwünschte $\mathrm{zu}$ unterbinden et vice versa, also welche Regelsysteme dazu weniger geeignet sind. Der Begriff der Institution ist dabei weit zu verstehen i.S.v. Regelsystemen, in denen bestimmte Verhaltensweisen verbindlich festgelegt sind. ${ }^{286}$ Damit unterfallen dem Begriff der Institution z.B. Märkte, Unternehmen, Verbände und die Gesellschaft selbst, letztlich aber auch Rechtsnormen, soziale Normen und Verträge. ${ }^{287}$ Institutionen werden daher im Rahmen der Neuen Institutionenökonomik als Systeme der Anreizsetzung und Sanktionierung von Gesellschaften zum Gegenstand der Analyse. ${ }^{288}$

Auf Grundlage dieses umfassenden Verständnisses des Begriffes Institution stellt die ökonomische Analyse des Rechts einen Teilbereich der Neuen Institutionenökonomik dar. Sie bewertet die Folgen der rechtlichen Rahmenbedingungen mit Hilfe des Kriteriums der ökonomischen Effizienz (sog. positive Theorie des Rechts) und macht in normativer Hinsicht Vorschläge, wie Recht zu gestalten ist, damit ökonomisch effiziente Ergebnisse erzielt werden können. Sie behandelt auch die Mechanismen, die zu Rechtsänderungen führen, ebenso untersucht sie die Funktionsweise der Justiz und mögliche Verbesserungen ihrer Funktionsfähigkeit. Dabei vermag ein solcher Ansatz sowohl normative Aussagen zu liefern als auch rechtstatsächlich testbare Modelle zur Verfügung zu stellen.

Ziel einer solchen normativen, aber auch empirischen Aufarbeitung des Verhältnisses von Mediation und traditionell prozessorientierter Konfliktbewältigung ist die Senkung volkswirtschaftlich nicht erwünschter Kosten der Konfliktlösung, die sowohl in Zeit- als auch personellem und sachlichen Kostenaufwand für die Justiz im weitesten Sinne, aber auch für die Beteiligten stehen. Hierfür muss der status quo in seinen Anreizstrukturen für die Verfahrensbeteiligten ebenso wie die Auswirkungen einer Mediation auf Motivation und Verfahrensverhalten untersucht werden.

Unterstützt wird eine solche Begleitforschung durch empirisch-quantitiative und qualitative Methoden, die die verdeckte Motivation der Verfahrensbeteiligten und ihr strategisches Verhalten aufdecken und einer Analyse zugänglich machen kann.

\footnotetext{
286 Eine einheitliche Definition des Begriffes existiert nicht. Die Offenheit des Begriffs soll eine zu frühe Einengung in dem insgesamt noch jungen Forschungsgebiet vermeiden, vgl. Janson, S. 21; Zu den Definitionsversuchen s. auch Richter/Furubotn, S. 7 f., Martiensen, S. 11 ff.

287 Martiensen, S. 11 ff, 25.

288 Vgl. auch North, 84 American Economic Review 359 (1994).
} 
174 Für dieses Vorgehen sind folgende Phasen zu unterscheiden:

175 In einem ersten Schritt werden die theoretischen Vorarbeiten zur Klärung der Anreizstrukturen für die an einer Konfliktbewältigung Beteiligten (Richterschaft, Anwälte, Konfliktparteien, Rechtsschutzversicherungen) geleistet.

176 Auf Grundlage des theoretischen Modells werden Fragebögen gemeinsam mit der sozialwissenschaftlichen Forschergruppe entwickelt. Anhand der empirischen Feldforschung wurde die tatsächliche Auswirkung der Mediation auf die Akzeptanz der Beteiligten untersucht.

177 Die empirischen Ergebnisse werden abschließend vor dem Hintergrund des erarbeiteten analytischen Rahmens ausgewertet und in normative Vorschläge umgewandelt.

\section{Methodologische Grundlagen}

178 Die ökonomische Analyse des Rechts ermittelt auf der Grundlage des ökonomischen Verhaltensmodells die Folgen, die durch rechtliche Regeln (und auch von Gerichtsentscheidungen) in der Rechtswirklichkeit ausgelöst werden und bewertet diese mit Hilfe des Kriteriums der ökonomischen Effizienz. Gleichzeitig vermag die ökonomischen Analyse des Rechts Vorschläge zu machen, wie Recht zu gestalten ist, damit ökonomisch effiziente Ergebnisse erzielt werden. Sie behandelt ferner die Mechanismen, die zu Rechtsänderungen führen, ebenso untersucht sie die Funktionsweise der Justiz und mögliche Verbesserungen ihrer Funktionsfähigkeit.

179 Methodologische Grundlage für die ökonomische Analyse des Rechts ist dabei das sog. „Ökonomische Paradigma“289, welches auf dem methodologischen Individualismus, der Knappheit der Ressourcen und dem immer wieder kontrovers diskutierten Verhaltensmodell des „homo oeconomicus“ basiert, welches das Eigennutztheorem sowie die Annahme rationalen Verhaltens voraussetzt.

\section{a) Methodologischer Individualismus}

180 Der methodologische Individualismus ${ }^{290}$ stellt das Individuum in den Mittelpunkt der Betrachtung, dieses wird als Ausgangspunkt und Träger aller Entscheidungen

\footnotetext{
${ }^{289}$ Vgl. hierzu beispielhaft Kirchner, Ökonomische Theorie des Rechts, S. 12 ff.

${ }^{290}$ Hierzu Behrens, Die ökonomischen Grundlagen des Rechts, S. 34 ff.; Kirchner, Ökonomische Theorie des Rechts, S. 18 ff.; Schwintowski, JZ 1998, 581, 583 f.; Coleman, Foundations of Social Theory, S. 5; Arrow 84 American Economic Review 1 ff. (1994).
} 
in einer Gesellschaft akzeptiert. Auch bei Organisationen sind es allein die Individuen, die einen Willen bilden können, da nur sie über Präferenzen verfügen. Und somit werden auch nur die individuellen Interessen und Bedürfnisse, nicht aber irgendein abstrakter Kollektivwille berücksichtigt. Zum anderen sieht der methodologische Individualismus nur natürliche Personen als in der Lage zum Handeln an. Damit kann und muss letztlich jedes auftretende kollektive Phänomen auf individuelles Verhalten zurückgeführt werden.

Der methodologische Individualismus bzw. die auf ihm basierende Theorie will zugleich aber nicht das tatsächliche Verhalten jeder einzelnen Person erklären können, vielmehr steht auch im Rahmen der ökonomischen Theorie des Rechts die Erklärung und Vorhersage des Verhaltens von aggregierten, also größeren Gruppen von Akteuren im Vordergrund. ${ }^{291}$

\section{b) Knappheit der Ressourcen}

Der Vollständigkeit des ökonomischen Paradigmas halber ist an dieser Stelle auf die Voraussetzung der Knappheit der Ressourcen kurz einzugehen. Diese Voraussetzung verdeutlicht, dass die zur Bedürfnisbefriedigung zur Verfügung stehenden Mittel begrenzt sind und wirft damit die Frage nach dem „Wirtschaften“ als ureigenstes Erkenntnisobjekt der Ökonomie auf. Hinzuweisen ist darauf, dass sich die Ressourcenknappheit nicht nur auf wirtschaftliche Güter im traditionellen Sinne, sondern auch auf immaterielle Güter und dabei insbesondere auch auf das Gut „Zeit“"292 bezieht.

Erst aufgrund dieses omnipräsenten Problems der Knappheit der Güter sind menschliche Wahlhandlungen bzw. ökonomische Entscheidungen zur Bedürfnisbefriedigung in allen Bereichen notwendig. Der ökonomische Ansatz wird damit $\mathrm{zu}$ einem allgemeinen entscheidungstheoretischen. ${ }^{293}$

\section{c) Verhaltensmodell des Homo oeconomicus}

Aufgrund der beiden genannten Voraussetzungen steht im Zentrum der Ökonomik das Individuum, das aufgrund der Knappheit der Güter dazu gezwungen ist, sich zwischen verschiedenen Handlungsalternativen zu entscheiden. Die Kriterien, nach denen diese Entscheidung getroffen wird, bestimmt das ökonomische Verhaltensmodell als ein positives, nicht normatives Verhaltensmodell, das von dem

${ }^{291}$ Kirchgässner, Homo Oeconomicus, S. 23 ff.

292 Behrens,ZfA 1989, 209, 212 spricht von der Zeit als wohl knappsten Ressource überhaupt.

${ }^{293}$ Behrens, RT 1981, 472, 474; Homann/Suchanek, Ökonomik: Eine Einführung, S. 60. 
Modell des homo oeconomicus ausgeht. Das Verhaltensmodell des homo oeconomicus beruht im Wesentlichen auf den Annahmen des Eigeninteresses und des Rationalverhaltens ${ }^{294}$ : Danach verfolgt der Mensch stets seine eigenen, meist egoistischen Interessen und geht bei der Verfolgung dieser Ziele rational vor. Ein rational verhaltender Akteur prüft in jedem Einzelfall seinen Nutzen aus den ihm zur Verfügung stehenden Handlungsalternativen mit Blick auf seine Präferenzen. Er wägt dann bei der Wahl von Handlungen den Nutzen und die Kosten gegeneinander ab und versucht so, seinen Nutzen unter den Nebenbedingungen bzw. Restriktionen zu maximieren, welche durch die bestehende institutionelle Ordnung vorgegeben sind. ${ }^{295}$

185 Hinsichtlich des Rationalverhaltens des Individuums ist zu berücksichtigen, dass das Individuum in vielen Situationen nicht unter Sicherheit, sondern unter Unsicherheit Entscheidungen treffen muss. In diesen Fällen weiß das Individuum nicht sicher, welche genauen Folgen sich aus einer Handlungsalternative ergeben, bzw. wie hoch der Nutzen sein wird, sondern das Individuum kennt nur die Wahrscheinlichkeiten bzw. versucht diese abzuschätzen, mit denen bestimmte Handlungen zu bestimmten Folgen, die Nutzen stiften können, führen. In diesen Fällen maximiert das Individuum nicht seinen tatsächlichen Nutzen, sondern nur noch seinen Erwartungsnutzen, also den mit der Eintrittswahrscheinlichkeit für ein bestimmtes Ereignis gewichteten Nutzen. ${ }^{296}$ Dies hat, wie sich zeigen wird, ${ }^{297}$ insbesondere für die Parteien eine große Relevanz. Der Ausgang eines Prozesses kann nicht immer vorhergesehen werden. Somit sind die Einschätzung der Prozessaussichten und die Auswirkungen der Prozessaussichten auf die zu erwartenden Kosten und Kostenverteilung von großer Relevanz für ihre Entscheidungen.

186 Allerdings enthält dieses Verhaltensmodell keine exogenen Annahmen über konkrete Ziele, sondern geht von einer Präferenzautonomie der Menschen aus. Die Individuen bestimmen also selbst, wie hoch der Nutzen und wie hoch die Kosten sind, so dass letztlich auch altruistisches Verhalten mit dem Modell eines eigennutzenmaximierenden Menschen vereinbar ist. Auch ist das Merkmal der Eigennützigkeit des ökonomischen Verhaltensmodells generell für alle Arten von Präferenzen, monetären wie auch immateriellen, offen.

${ }^{294}$ Hierzu ausführlich: Janson, S. 26 ff., zur Diskussion um dieses Verhaltensmodell sieher auch Homann/Suchanek, S. 414 ff.; van Aaken, S. 73 ff.

295 Kirchner, Ökonomische Theorie des Rechts, S. 9; Homann/Suchanek, S. 414.

${ }^{296}$ Janson, S. 39; Martiensen, S. 150 f.

${ }^{297}$ Siehe hierzu insbesondere unten Rn. 289, 334. 
Die in der jeweiligen Situation erwarteten Nutzenerwartungen bezeichnet man, wenn und sofern sie das Handeln bestimmen, als Anreize i.S.v. situationsbedingten handlungsbestimmenden Vorteilserwartungen. Das Standardmodell der ökonomischen Handlungstheorie formuliert daher, dass Individuen den Anreizen der Situation folgen. ${ }^{298}$

Individuen reagieren daher nach diesem Modell auch auf rechtliche Regeln und gerichtliche Standards rational und eigennützig. Das Recht beeinflusst Nutzen und/oder Kosten von Handlungen und entfaltet somit verhaltenssteuernde Wirkungen.

\section{Theoretisches Modell der Anreizstrukturen}

Basierend auf diesen methodologischen Grundlagen sind im Folgenden in einem theoretischen Modell die Anreizstrukturen für die an einer Konfliktbewältigung Beteiligten (Richterschaft, Anwälte, Konfliktparteien, Rechtsschutzversicherungen) zu klären. Dabei ist zum einen aufzuarbeiten, wie die Anreizstrukturen aufgrund der rechtlichen und tatsächlichen Rahmenbedingungen während des Modellprojektes ausgestaltet sind, zum anderen ist zu analysieren, in wiefern aus normativer Perspektive rechtspolitischer Handlungsbedarf besteht, um bei einer langfristigen Aufnahme der Gerichtsnahen Mediation in das Leistungsangebot der Justiz mediationsfördernde Anreize für alle Beteiligten zu setzen.

Die folgende Untersuchung bezieht sich zunächst auf das zivilgerichtliche Verfahren. Soweit für die anderen Gerichtsbarkeiten des Modellprojektes (Verwaltungsgericht Hannover und Sozialgericht Hannover) rechtliche Besonderheiten bestehen, wird darauf jeweils gesondert hingewiesen und auf etwaige Auswirkungen dieser Besonderheiten auf die rechtsökonomische Analyse eingegangen.

298 Homann/Suchanek, S. 32. 


\section{Anreizstrukturen Richterschaft ${ }^{299}$}

\section{a) Richter als Fallmanager}

191 Nach Einreichung der Klage sollen sämtliche Richter an den Projektgerichten als Fallmanager anhand des Aktenstudiums entscheiden, ob sich der vorliegende Fall für eine Mediation eignet, und gegebenenfalls den Parteien und ihren Anwälten die gerichtsnahe Mediation vorschlagen. Nach dem in Göttingen praktizierten Ansatz erfolgt eine Abgabe an den Richtermediator jedoch auch ohne gesonderte Prüfung der Mediationseignung des Falles.

Die Bereitschaft des Fallmanagers zur Abgabe von Fällen an den Richtermediator ist somit wesentliche Voraussetzung für die gerichtsnahe Mediation. Es ist daher zunächst danach zu fragen, welche Anreize für den Fallmanager bestehen, geeignete Fälle auszuwählen, den beteiligten Parteien die Mediation vorzuschlagen und den Fall an den Richtermediator abzugeben.

\section{aa) Richterliche Unabhängigkeit}

192 Für die folgende Betrachtung ist zunächst die besondere verfassungsrechtliche Stellung des Richters zu berücksichtigen. Den Richtern wird durch das Grundgesetz sowohl eine sachliche Unabhängigkeit (vgl. Art. 97 Abs. I GG; Art. 20 III GG) von Weisungen und Empfehlungen der Exekutive und Legislative gewährleistet, als auch eine persönliche Unabhängigkeit (Art. 97 Abs. 2 GG), die die sachliche absichern soll. Diese verfassungsrechtlich garantierte äußere Unabhängigkeit ist zugleich Ausdruck einer besonderen Form von Bindung: Da der Richter gem. \97 I GG und Art. 20 III GG nur an Gesetz und Recht gebunden sein soll, muss er von anderen Einflüssen freigehalten werden. Der unabhängigen Stellung des Richters entsprechen besondere Pflichten, die im Richtereid (vgl. \38 bzw. \ 45 DRiG) zum Ausdruck kommen. Er hat getreu dem Grundgesetz und getreu den Gesetzen nach bestem Wissen und Gewissen ohne Ansehen der Person zu

\footnotetext{
${ }^{299}$ In der zumeist US-amerikanischen Literatur wird richterliches Verhalten fast ausschließlich hinsichtlich der Entscheidungsfunktion bzw. der rechtsprechenden Funktion des Richters im Prozess untersucht. Die dort erarbeiteten Anreizstrukturen und Einflussfaktoren des richterlichen Verhaltens sind auf die Aufgaben des Fallmanagers, die Mediationseignung des Falles zu bestimmen und Fälle an die Richtermediatoren abzugeben, nur sehr beschränkt übertragbar und verwertbar. Für eine rechtsökonomische Untersuchung des Richtermediators zeigen sich wegen der grundsätzlichen Verschiedenheit der Aufgaben des rechtsprechenden und mit Entscheidungsgewalt ausgestatteten Richters und des Richtermediators, dem anders als im gerichtlichen Verfahren keine Entscheidungsbefugnis zukommt, sondern der auf die Aufgabe einer unparteilichen und neutralen Verhandlungsmoderation beschränkt ist, noch größere Einschränkungen bei der Verwertung und Übertragbarkeit der Ergebnisse der rechtsökonomischen Literatur.
} 
urteilen und nur der Wahrheit und Gerechtigkeit zu dienen. Ferner hat sich der Richter innerhalb und außerhalb seines Amtes, auch bei politischen Betätigungen, so zu verhalten, dass das Vertrauen in seine Unabhängigkeit nicht gefährdet wird (vgl. \ $39 \mathrm{DRiG}$ ). Der Richter ist also bei seinen Handlungen der Gerechtigkeit und Wahrheit, dem Grundgesetz und anderen Gesetzen verpflichtet. ${ }^{300}$ Diese rechtliche Stellung des Richters zielt darauf ab, zu verhindern, dass der Richter positive Auszahlungen von einer Partei dafür erhält, in einer bestimmten Art und Weise im Streitfall zu entscheiden; zum anderen soll der politische Einfluss bestimmter Interessengruppen auf seine Entscheidungen minimiert werden. ${ }^{301} \mathrm{Um}$ diese unabhängige Position des Richters zu gewährleisten und eine (direkte) Einflussnahme Dritter auszuschließen, besteht durch die Bezüge in Form eines festen Gehalts auch eine finanzielle Unabhängigkeit. Daher gilt grundsätzlich für den Fallmanager-Richter, dass ihm - anders als sonstigen Personen des politischen Lebens, die nach der Theorie des Public Choice auf ihre Eigennutzmaximierung überprüft werden - wegen seiner finanziellen und politischen Unabhängigkeit für sein Handeln und seine Entscheidungen keine direkten Präferenzen unterstellt werden können und er daher vor allem auf indirekte Anreize nichtpekuniärer Art beschränkt ist. ${ }^{302}$

\section{bb) Arbeitszeitersparnis}

\section{(1) Konzentration auf wesentliche streitige Verfahren}

Da der Richter finanziell unabhängig ist und ein festes Gehalt bezieht, gibt es keine monetären Anreize für ihn, in einer bestimmten Art und Weise tätig zu werden oder besonders leistungsorientiert zu arbeiten. Um allerdings trotz fehlender finanzieller Anreize für die Richter die Funktionsfähigkeit der Justiz zu gewährleisten, hat der Richter eine bestimmte Anzahl von Fällen innerhalb seiner Arbeitszeit zu verhandeln, die von dem Deputatsschlüssel vorgegeben ist.

Für die gerichtsnahe Mediation ist mit Blick auf den Deputatsschlüssel zu beachten, dass die in der Mediation erfolgreich verhandelte Vereinbarung in der Regel wieder vor dem Fallmanager als gesetzlichem Richter als Prozessvergleich proto-

\footnotetext{
300 Posner, Economic Analysis of Law, S. 581 hingegen betont, dass auch Richter in erster Linie wie alle anderen Individuen versuchen, ihren persönlichen Nutzen zu maximieren; ebenso Landes/Posner, 9 Journal of Legal Studies 367, 369 (1980); Cohen, 7 Journal of Law, Economics, and Organization 183, 184 (1991).

301 Posner, Economic Analysis of Law, S. 581.

302 Alexander, 23 Journal of Legal Studies 647, 648 (1994); Cohen, 12 International Review of Law and Economics 12 (1992); Babusiaux, in: Schmidtchen/Weth, Der Effizienz auf der Spur, S. 157, 171.
} 
kolliert und auf den Deputatsschlüssel des Fallmanagers vollständig angerechnet wird. Damit besteht für den Fallmanager-Richter die Möglichkeit, durch Abgabe von mediationsgeeigneten Fällen in die gerichtsnahe Mediation Arbeitszeit einzusparen und dennoch die Verpflichtungen aus dem Deputatsschlüssel zu erfüllen. Damit bleibt für den einzelnen Richter mehr Zeit für die nicht mediationsgeeigneten bzw. die in der Mediation gescheiterten Fälle, die sich durch rechtliche Probleme und Beweisschwierigkeiten auszeichnen. Er kann sich so auf die Lösung dieser rechtlich komplexen Fälle oder auf Präzedenzfälle konzentrieren und somit eine Erhöhung der Qualität der Justizdienstleistung bewirken.

\section{(2) Reduzierung des Arbeitsaufwandes}

195 Ist aufgrund eines festen Gehalts eine direkte Erhöhung des Einkommens nicht möglich, so können aber gleichwohl eigene Interessen, wie z.B. die möglichst wenig arbeitsintensive und möglichst zügige Erledigung eines Verfahrens auch bei festen Gehaltsbezügen, verfolgt werden. 303

196 Unter diesem Aspekt könnte bei vollständiger Anrechnung der später als Prozessvergleich protokollierten Abschlussvereinbarung auf den Deputatsschlüssel die Gefahr des strategischen Verhaltens einzelner Richter bestehen. Einzelne Richter könnten versuchen, durch die Abgabe von Fällen in die Mediation ihre persönliche Arbeitszeit und ihren persönlichen Arbeitsaufwand zu reduzieren, ohne die eingesparte Zeit für andere Fälle zu verwenden.

197 Es könnte dann insbesondere bei komplizierten und komplexen Fällen, die sich durch rechtliche Probleme und Beweisschwierigkeiten auszeichnen und somit einen hohen Zeit- und Arbeitsaufwand erfordern, ein solcher Anreiz zur Abgabe des Falles in die Mediation bestehen. Einfache und weniger komplexe Fälle könnten dann von einzelnen Richtern strategisch zurückgehalten werden, wenn ein schneller Abschluss des Falles möglich erscheint und auch so der Deputatsschlüssel erfüllt werden kann.

303 Dürschke, SGb 2001, 532, 533; zur Steigerung der persönlichen Freizeit als indirektem Teil des Arbeitseinkommens siehe auch Posner, Economic Analysis of Law, S. 582; Posner, What do judges maximize? (The same thing everybody else does), Chicago Working Paper in Law \& Economics No. 15 (2 ${ }^{\text {nd }}$ series), S. 8; Landes/Posner, 9 Journal of Legal Studies 367, 369 (1980); Macey, 23 Journal of Legal Studies 627, 631 (1994); Cohen, 7 Journal of Law, Economics, and Organization 183, 184 (1991); Fucik, in: Bundesministerium für Justiz/Lewisch/Rechberger, 100 Jahre ZPO: ökonomische Analyse des Zivilprozesses, S. 191, 193; Lande, UCLA Law Review 69 (2000), 69, 124; kritisch zu dem Anreizkriterium der Arbeitsersparnis s. Alexander, 23 Journal of Legal Studies 647, 648 (1994). 
Fraglich ist, ob die an den Richtermediator abgegebenen Fälle dann allein nach dem Kriterium der persönlichen Arbeitsersparnis und nicht nach dem Kriterium der Eignung für die Mediation ausgewählt würden. Unabhängig von der Frage, welche Fälle mediationsgeeignet sind, ist auch mit Blick auf die Möglichkeit der Arbeitszeitersparnis davon auszugehen, dass offensichtlich ungeeignete Fälle nicht abgegeben werden, da diese Fälle nach einem eventuellen Scheitern der Mediation wieder vor dem Fallmanager streitig verhandelt werden müssten. Dann aber bestünde eventuell ein psychischer Druck von den Parteien auf den Richter, bzw. die Reputation des Richters und auch der Justiz im Allgemeinen würde darunter leiden.

Bei der derzeitigen vollen Anrechnung eines in der Mediation verhandelten Prozessvergleiches auf den Deputatsschlüssel könnte somit zwar für den Fallmanager - wie oben aufgezeigt - einen Anreiz bestehen, sich und seine Arbeitskraft/-zeit auf die Fälle zu konzentrieren, die eine umfassende rechtliche Würdigung oder eine gerichtliche Entscheidung erfordern, doch zugleich stellt dies aber eine Gefahr dar, dass durch die vollständige Anrechnung dieser Prozessvergleiche ein Anreiz für einige Richter bestehen könnte, möglichst komplexe und zeitaufwändige Fälle an die Richtermediatoren unabhängig von ihrer Mediationseignung abzugeben. Eine Erfüllung des Deputatsschlüssels wäre dann für die Fallmanager unter minimalem Aufwand möglich.

\section{cc) Gefahr eines Kompetenzverlustes}

Gibt der Fallmanager die Fälle an den Richtermediator ab und setzt er das Verfahren bis zur Protokollierung der Abschlussvereinbarung als Prozessvergleich aus, so gibt der Fallmanager die Entscheidungsgewalt über das Verfahren ab. Für ihn besteht durch die Abgabe von Fällen an den Richtermediator die Gefahr des Kompetenzverlustes und somit auch die des Verlustes an interessanten und wichtigen Fällen. ${ }^{304}$ Ein Streben nach Beibehalten von Entscheidungskompetenz ${ }^{305}$ und von interessanten und wichtigen Fällen kann der oben beschriebenen Gefahr eines bloßen Anreizes zur Arbeitszeitminimierung zwar entgegen wirken, allerdings würde dann erneut die Gefahr bestehen, dass der Fallmanager die Fälle auch nur nach seinen persönlichen Präferenzen und nicht nach dem Kriterium der Mediationseignung auswählt.

\footnotetext{
304 Siehe zum Streben des Richters nach interessanten und wichtigen Fällen Alexander, 23 Journal of Legal Studies 647, 663 (1994); ferner schreibt Posner, Economic Analysis of Law, S. 582; Posner, What do judges maximize? (The same thing everybody else does), Chicago Working Paper in Law \& Economics No. 15 (2 $2^{\text {nd }}$ series), S. 10 dem Richter auch ein Streben nach Prestige und Macht zu. ${ }^{305}$ Landes/Posner, 9 Journal of Legal Studies 367, 369, 370 (1980).
} 
201 Auch könnte gegen eine Abgabe von Fällen an den Richtermediator das richterliche Selbstverständnis stehen, als berufener Entscheider den Konflikt mit Mitteln des Rechts lösen zu können. Gegebenenfalls könnten Richter befürchten, durch die Abgabe ein inkompetentes Bild bei den Kollegen zu vermitteln.

\section{dd) Beförderungschancen ${ }^{306}$}

202 Gem. Art. 33 GG Abs. 2 hat jeder Deutsche nach seiner Eignung, Befähigung und fachlichen Leistung den gleichen Zugang zu jedem öffentlichen Amt. Diese Regelung gilt auch für die richterlichen Beförderungsämter. Die Gewichtung dieser drei genannten Kriterien, Eignung, Befähigung und fachliche Leistung, wird dem pflichtgemäßen Ermessen des Dienstherrn überlassen und unterliegt einem weiten, gerichtlich nicht in vollem Umfang nachprüfbaren Beurteilungsspielraum der Beurteilungsbehörde bzw. des Dienstherren. ${ }^{307}$ Die fachliche Leistung ist zumeist ausschlaggebend für die Beförderungschancen des Richters. Die Tätigkeit des Richters als Fallmanager könnte auch Einfluss auf seine fachliche Leistung haben. Zum einen ist die gerichtsnahe Mediation in das Leistungsspektrum der Justiz in Niedersachsen (zumindest im Rahmen des Modellversuches) aufgenommen worden. Daher kann auch eine fachliche Leistung festgestellt werden, die sich auf die Mitarbeit im Rahmen der gerichtsnahen Mediation bezieht. Dazu zählt auch die Auswahl für die gerichtsnahe Mediation geeigneter Fälle. Zum anderen bietet sich durch die Möglichkeit der gerichtsnahen Mediation die Möglichkeit für den Fallmanager, mediationsgeeignete Fälle abzugeben und sich auf die Fälle zu konzentrieren, die sich durch rechtliche Probleme auszeichnen (s.o.). Dies kann sich ebenfalls positiv auf die fachliche Leistung des Richters auswirken. Der Fallmanager-Richter kann daher durch die Abgabe von geeigneten Fällen in die gerichtsnahe Mediation seine Beförderungschancen erhöhen.

203 Gleichwohl ist ein pauschales Urteil nicht möglich. Ist die Gewichtung der drei genannten Kriterien, Eignung, Befähigung und fachliche Leistung, dem pflichtgemäßen Ermessen des Dienstherrn überlassen, so kann z.B. auch die Fähigkeit zum Interessenausgleich bedeutsam sein und durch die Anzahl der Vergleiche nachgewiesen werden. In diesen Fällen würde dann die Gefahr des Zurückbehaltens von mediationsgeeigneten Fällen bestehen, die sie sich auch durch einen richterlichen Vergleich lösen ließen, um so die eigenen Vergleichszahlen und somit die Beförderungschancen zu erhöhen.

\footnotetext{
306 Zu Beförderungschancen als Anreiz richterlicher Tätigkeit s. Cohen, 12 International Review of Law and Economics 13, 16 f. (1992); Cohen, 7 Journal of Law, Economics, and Organization 183, 185 (1991).

307 BVerwGE 11, 139, 139 f.; 15, 39, 40; 68, 109, 110.
} 
Bei einer langfristigen Installation der Gerichtsnahen Mediation würde diese Gefahr jedoch reduziert werden, wird doch dann die Gerichtsnahe Mediation in den Leistungskatalog der Justiz aufgenommen. Die Förderung der Gerichtsnahen Mediation durch die Abgabe von Fällen, die auch vergleichsgeeignet sind, kann dann hinsichtlich der Beförderungschancen nicht negativ bewertet werden.

\section{ee) Ausblick: Rechtspolitische Handlungsempfehlungen}

Langfristig sind die Rahmenbedingungen mit Blick auf den Fallmanager zu ändern, um die eben aufgezeigte Gefahr einer bloß arbeitsaufwand-motivierten Abgabe von Fällen in die Mediation ungeachtet ihrer Eignung zu vermeiden.

Zunächst ist dabei an eine Änderung des Deputatsschlüssel dergestalt zu denken, dass eine später als Prozessvergleich protokollierte Vereinbarung, die in der Mediation verhandelt wurde, nicht vollständig auf den Deputatsschlüssel anzurechnen ist, um den Zeitaufwand für normale streitige Verfahren in ein angemessenes Verhältnis zu der (bloßen) Protokollierung der Abschlussvereinbarung der Mediation als Prozessvergleich zu setzen (z.B. hälftige Anrechnung). Hierbei ist jedoch auch zu beachten, dass sich während des Modellprojekts die Praxis durchgesetzt hat, dass der Richtermediator als ersuchter Richter gem. SS 278 Abs. 5 S.1, 362 ZPO analog die Mediationsvereinbarung protokolliert und hierdurch für den abgebenden gesetzlichen Richter kein weiterer Arbeitsaufwand mehr anfällt. Eine anteilige Anrechnung, die sich an dem Verhältnis des Arbeitsaufwandes von gesetzlichem Richter und Richtermediator orientiert, ist daher wenig zweckdienlich Bei der Ausgestaltung eines Anrechnungssystems ist ferner zu beachten, dass keine negativen Anreize gesetzt werden: Ist der Anrechnungsfaktor zu gering, besteht kein Anreiz für den Fallmanager mehr, den Fall an den Richtermediator abzugeben (abgesehen von äußerst aufwendigen Verfahren), um sich auf andere Verfahren konzentrieren zu können. Hier gilt es zu beachten, dass der Richtermediator auf die Zusammenarbeit mit dem Fallmanager angewiesen ist, um überhaupt in Kontakt mit den Parteien und deren Anwälte zu treten.

Ferner ist durch die Erarbeitung eines Kriterienkataloges im Rahmen der Begleitforschung des Modellprojektes auch die Unsicherheit der Fallmanagers über die Mediationseignung der Fälle und somit zugleich die Gefahr zu reduzieren, dass die Fälle für die Mediation nach persönlichen Interessen und nicht allein nach ihrer Mediationseignung ausgewählt werden.

Erweist sich der in Göttingen praktizierte Ansatz als erfolgversprechend, so ist auch über diese Alternative nachzudenken. Nach diesem Modell - Abgabe des Falles an den Richtermediator ohne Prüfung des Mediationseignung des Falles; Fälle werden dann als mediationsgeeignet angesehen, wenn die Parteien noch 
bereit sind, miteinander zu reden - erscheint die Erarbeitung eines Kriterienkataloges als nicht zwangsläufig notwendig. Vielmehr wohnt diesem Modell eine Tendenz zu einer grundsätzlich vorgeschalteten Mediation inne. Erfolgt die Abgabe von Fällen an den Richtermediator ohne eine gesonderte Prüfung der Mediationseignung des Falles, so ließe sich über die Einführung einer Mediationspflicht nachdenken bzw. über Kostenvorteile für die Parteien bei der Wahrnehmung einer gerichtsnahen Mediation vor dem normalen Verfahren (bzw. Kostennachteilen bei Ablehnung der gerichtsnahen Mediation und bei Fehlen eines Nachweises eines anderen außergerichtlichen Streiteinigungsversuches). Bei der Einführung einer generell vorgeschalteten Mediation entfielen auch die oben genannten Bedenken strategischen Verhaltens einzelner Fallmanager, da die Abgabe des Falles in die Gerichtsnahe Mediation dann von ihm unabhängig erfolgen würde. Eine Pflicht zur Mediation sollte aber aufgrund der der Mediation wesensimmanenten Freiwilligkeit besser dahingehen verstanden werden, dass generell ein Informationsgespräch mit den Konfliktparteien über die gerichtsnahe Mediation eingeführt werden könnte; zumindest in den Fällen, in denen die Parteien nicht bereits einen anderen außergerichtlichen Streitschlichtungsversuch vorweisen können. Die generelle Einführung eines Informationsgesprächs würde zudem auch die Akzeptanz des Mediationsangebots innerhalb der Gerichte und der Justiz stärken bzw. Vorbehalte in der Richterschaft, die sich auch in einer nur zögerlichen Bereitschaft zur Fallabgabe äußern könnte, abbauen.

209 Wird eine „Pflicht“ zu Mediation nicht angeordnet bzw. generell ein Informationsgespräch vorgeschaltet, sondern soll es auch weiterhin im Ermessen des Richters liegen, ob ein Fall in die Mediation abgegeben wird, so bietet das derzeitig praktizierte Modell in Göttingen für die nähere Ausgestaltung auch hier einen Ansatzpunkt: ${ }^{308}$ Die in der Mediation erzielten Vergleiche zählen auch weiterhin vollständig zugunsten der abgebenden Kammer. Gleichzeitig sieht aber die richterliche Geschäftsverteilung eine erhöhte Zuweisung von Verfahren an die Kammer vor, die sich dann in selbst bestimmtem Umfang durch die Abgabe an die Mediationsabteilung entlasten kann. Hierdurch wird ein sehr starker Anreiz geschaffen, Fälle in die Mediation abzugeben und so eventuell für die Justiz Einsparpotentiale an richterlicher Arbeitszeit nutzbar zu machen. Im Gegenzug wurden die Richtermediatoren aus den Kammern abgezogen und mit ihrem für die gerichtsinterne Mediation notwendigen Arbeitsanteil in den Kammern entlastet. Eine Mehrbelastung für die Kollegen ist dadurch aber, wie die Erfahrung am LG Göttingen zeigt, nicht entstanden; vielmehr tritt bei hohen Erledigungszahlen aufgrund der gerichtsinternen Mediation letztlich ein Entlastungseffekt zugunsten

308 Hierzu Götəv v. Olenbusen, ZKM 2004, 104, 105 f. 
der richterlich tätigen Kollegen ein, was wiederum die Abgabebereitschaft der Kollegen aufgrund der positiven Erfahrungen stärken kann. ${ }^{309}$

Im Ergebnis steht aber eine nicht vollständige, sondern nur anteilige Anrechnung eines Prozessvergleichs für den den Fall abgebenden gesetzlichen Richter oder die abgebende Kammer bei gleichem Pensenschlüssel einer Erhöhung der zu erledigenden Fallzahlen bei vollständiger Anrechnung gleich. Hierbei handelt es sich dann letztlich nur um Unterschiede in der rechnerischen Ausgestaltung.

\section{b) Richtermediatoren}

Mediation wird in der Literatur häufig als eine win-win-Situation ${ }^{310}$ im spieltheoretischen Sinne bezeichnet, die gegenüber dem status quo und einem richterlichen Urteil eine pareto ${ }^{311}$-überlegene Lösung hervorzubringen vermag, also als kooperatives Spiel mit positiver Gesamtauszahlung bewertet wird. ${ }^{312} \mathrm{Im}$ Unterschied zur herkömmlichen Grundsituation eines solchen Spiels ist dabei zu beachten, dass der Mediator ein dritter Spieler ist, der aber - dem Prinzip nach - an nichts anderem interessiert ist, als die Parteien bei einer konsensualen Konfliktlösung unparteiisch zu unterstützen. Eine Koalitionsbildung des Mediators mit der einen oder anderen Seite würde hingegen die Situation „kippen“ lassen und in ein herkömmliches Nullsummen-Spiel ${ }^{313}$ verwandeln. ${ }^{314}$

Für den Richtermediator gilt in der gerichtsnahen Mediation nun insbesondere, dass ihm, anders als im gerichtlichen Verfahren, keine Entscheidungsbefugnis zukommt, sondern er auf die Aufgabe einer unparteilichen und neutralen Verhandlungsmoderation beschränkt ist.

Da dem Richtermediator wie eben beschrieben eine entscheidende Rolle für das Gelingen der Mediation zukommt, sind nun die Rahmenbedingungen und Anreize

309 Siehe hierzu auch unten Rn. 195 und Rn. 205.

310 Im Gegensatz zu einem Nullsummenspiel, bei dem bei jedem möglichen Ereignis ein Spieler eine Auszahlung erhält, die der andere Spieler bezahlen muss, bezeichnet eine win-win-Situation in der Spieltheorie einen Zustand, in der beide Spieler, d.h. also die Parteien, positive Auszahlungen erhalten.

311 Eine Regelung wird dann pareto-effizient genannt, wenn sie aus der Sicht der Parteien nicht mehr dergestalt verbessert werden kann, dass zumindest eine Partei noch einen Vorteil erzielt, ohne dass die andere Partei hierdurch einen Nachteil erleidet; vgl. Schumann/Meyer/Ströbele, Grundzüge der mikroökonomischen Theorie, S. 35 f.

312 Weigel, in: FS Ott, S. 491, 492; Göthlich, IDR 2005, 68, 72.

313 Bei dem Nullsummenspiel erhält bei jedem Ereignis ein Spieler eine Auszahlung, die der andere Spieler zahlen muss.

314 Weigel, in: FS Ott, S. 491, 492. 
seines Handelns im Interesse der Parteien an einer unparteilichen und neutralen Verhandlungsmoderation zu klären.

\section{aa) Unabhängigkeit und Unparteilichkeit des Richtermediators}

214 Der Richtermediator wird im Rahmen der gerichtsnahen Mediation nicht rechtsprechend tätig, so dass das Gebot der richterlichen Unabhängigkeit (vgl. Art. 97 Abs. 1 und 2 GG, Art. 20 Abs. 3, sowie \39 bzw. \45 DRiG) im Bereich der gerichtsnahen Mediation nicht gilt. Eine Neutralitätspflicht des Richtermediators ergibt sich gleichwohl nicht nur aus der vertraglichen Vereinbarung mit den Konfliktparteien, sondern bereits unmittelbar aus der Verfassung, nämlich aus dem Recht auf ein faires Verfahren gemäß Art. 2 Abs. 1 GG in Verbindung mit dem Rechtsstaatsprinzip. Denn Art. 2 Abs. 1 GG in Verbindung mit dem Rechtsstaatsprinzip gewährt dem Einzelnen auch außerhalb der Anwendbarkeit der Prozessgrundrechte ein Recht auf ein faires, rechtsstaatliches Verfahren,315 was in jedem Fall die Neutralität des in einem Konfliktlösungsverfahren tätigen Dritten mit einschließt. Die Tätigkeit des richterlicher Mediator im Rahmen eines gerichtsnahen Mediationsverfahrens ist daher auch als solche keinen Grund zur Selbstablehnung in einem nach Beendigung der Mediation fortzusetzenden Klageverfahren; vielmehr bleibt die Besorgnis der Befangenheit aufgrund der im Mediationsverfahren zur Kenntnis des Richters gelangten vertraulichen Informationen stets eine Frage des Einzelfalls. 316

\section{bb) Fehlen finanzieller Anreize}

215 Der Richtermediator bezieht, wie auch die Fallmanager-Richter, ein festes Gehalt, das vom Staat und nicht von den Parteien bezahlt wird. Seine Bezahlung ist daher auch nicht von dem Erfolg und der beiderseitigen Zufriedenheit der Parteien mit der Mediation abhängig. ${ }^{317}$ Insbesondere kann somit nicht sein Beitrag und sein Bemühen um den Erfolg der Mediation kontrolliert werden. Zwar fehlt damit ein Kontrollmechanismus für die Parteien, doch bedeutet dies aber auch zugleich, dass der Richtermediator - anders als im Falle einer vereinbarungshöhenabhängigen Bezahlung eines privatwirtschaftlich tätigen Mediators - keinen Anreiz dazu hat, den Wert der Vereinbarung zum Nachteil der Parteien künstlich in die Höhe zu treiben.

\footnotetext{
315 BVerfG JZ 2000, 783 f.

316 LSG Niedersachsen-Bremen, ZKM 2005, 139 f.

317 Siehe dazu Weigel, in: FS Ott, S. 491, 495.
} 
Während die rechtsprechende Tätigkeit des Richters in formeller und materieller Hinsicht gerichtlich überprüfbar ist, wird sich eine Überprüfung der vermittelnden Tätigkeit des Richtermediators als Verhandlungsmoderator - abgesehen von groben Verstößen gegen den Grundsatz der Unparteilichkeit und Neutralität - als sehr schwierig erweisen. Die gerichtsnahe Mediation zielt nicht auf eine allein rechtliche Bewertung und Entscheidung von Streitigkeiten ab, sondern auf eine konsensuale Lösung, die den Konflikt unter Einbeziehung auch anderer Faktoren als der Rechtslage dauerhaft zu lösen und Parteibeziehungen aufrecht zu erhalten versucht, die sich stark in der Zufriedenheit beider Parteien mit dem Verfahren und Ergebnis der Mediation widerspiegelt.

Da die Bezahlung des Richtermediators nicht an die beiderseitige Zufriedenheit der Parteien geknüpft ist, sondern von dritter Seite erfolgt, und dadurch eine Kontrolle der Qualität seiner Mediatorenleistung durch die von seinem Handeln unmittelbar betroffenen Parteien durch pekuniäre Anreize nicht erfolgt, ist zu überlegen, ob und wie eine Kontrolle der Mediatorenleistung dennoch möglich ist, insbesondere ob der Mediator vorrangig im Interesse der beteiligten Parteien handelt. Dabei genügt es allerdings nicht, allein auf das Zustandekommen (irgend)einer Einigung in der Mediation abzustellen.

Fraglich ist, ob dieses Problem eines fehlenden finanziellen Anreizes für die Qualität der Mediatorentätigkeit über einen Reputationsmechanismus abgeschwächt wird. Für den privatwirtschaftlich tätigen Mediator gilt im Allgemeinen, dass die beiderseitige Zufriedenheit der Konfliktparteien Auswirkungen auf seinen Ruf und seine Reputation auf dem Markt für Mediatorentätigkeiten hat. Weitere Beauftragungen als Mediator hängen von seiner Reputation ab. Der privatwirtschaftlich tätige Mediator hat damit einen (indirekt auch finanziellen) Anreiz, für beide Parteien im zufrieden stellenden Sinne zu handeln. ${ }^{318}$

Dieser Reputationsmechanismus ist jedoch auf den Fall des Richtermediators nicht undifferenziert übertragbar. Schließlich wird dieser nicht von den Konfliktparteien beauftragt, sondern ihm werden die Fälle von dem gesetzlichen Richter/Fallmanager übertragen. Damit aber auf dem Markt für Mediatorendienstleistungen die Justiz mit dem Angebot der Gerichtsnahen Mediation mit privaten und etablierten Mediationsdienstleistern konkurrieren kann, ist an eine „Zertifizierung der Richtermediatoren“ zur Gewährleistung von Qualitätsstandards zu denken. Damit könnte die Justiz auf dem Markt für die Mediationsnachfrager ein positives Signal setzen, dass die Tätigkeit der Richtermediatoren Qualitätsstandards Genüge leistet. Im Innenverhältnis Justiz/Dienstherr und

318 Weigel, in: FS Ott, S. 491, 495. 
Richtermediator müssen dann neben einer umfassenden Ausbildung für die Richtermediatorentätigkeit auch regelmäßige Überprüfungen der Tätigkeit bzw. Weiterbildungen/Supervisionen eingerichtet werden. Hier kommt auch eine freiwillige Selbstbindung der Richtermediatoren an den europäischen Verhaltenskodex für Mediatoren ${ }^{319}$ in Betracht.

220 Darüber hinaus können niedrige Erfolgsquoten aber auch die Abgabebereitschaft der gesetzlichen Richter schwächen, da sie gegebenenfalls bestimmte Richtermediatoren für nicht ausreichend kompetent halten könnten, eine Einigung herbeizuführen. Ähnlich könnten auch Anwälte reagieren: Hohe Erfolgsquoten fördern die Bereitschaft der Anwälte, ihren Mandanten zur gerichtsnahen Mediation zu raten. Umgekehrt könnten auch hier niedrige Erfolgsquoten zu gewissen Vorbehalten gegenüber dem Mediationsangebot führen, da die Anwälte von einem Scheitern ausgehen und aufgrund der Gefahr zusätzlicher Arbeit ihren Mandanten von der gerichtsnahen Mediation abraten könnten. Daher ist davon auszugehen, dass die Reputation in den Augen der gesetzlichen Richter und Anwälte für den Richtermediator einen starken Anreiz setzt.

221 Schließlich könnte auch eventuell die richterliche Autorität darunter leiden, wenn der Richter nicht nur Entscheider ist, der in jedem Fall eine rechtliche Lösung des Konflikts findet und so den Konflikt endgültig beendet, sondern nun auch als Richtermediator als Vermittler tätig ist. Hier besteht immer die Gefahr, dass die Mediationsverhandlungen scheitern können. ${ }^{320} \mathrm{Ihm}$ könnte dann mangelnde Konfliktlösungskompetenz unterstellt werden, die auch auf die Tätigkeit als gesetzlicher Richter übergreift. Um die Reputation - auch für Richter mit Entscheidungsmacht - nicht zu schädigen, besteht daher für den Richtermediator ebenfalls ein Anreiz, ${ }^{321}$ möglichst gut im Mediationsprozess zu vermitteln und eine beide Parteien zufrieden stellende Lösung zu fördern.

\section{cc) Arbeitszeitersparnis}

\section{(1) Entscheidung für die Tätigkeit als Richtermediator}

222 Die Tätigkeit als Richtermediator könnte im Vergleich mit der normalen richterlichen, rechtsprechenden Tätigkeit vorteilhaft sein. Durch die Mediation entfällt das häufig langwierige Aktenstudium; die Behandlung von komplexen, rechtlich kom-

\footnotetext{
319 In der englischen Originalfassung abgedruckt in ZKM 2004, 148 f., eine deutsche Übersetzung findet sich im mediations-report 8/2004, S. 2 f.

320 Gottwald, AnwBl 2000, 265, 271.

321 Cohen, 7 Journal of Law, Economics, and Organization 183, 184 (1991).
} 
plizierten und/oder durch Beweisschwierigkeiten geprägten Fällen wird vereinfacht und kann zeitlich verkürzt werden, indem die Parteien selbst den Verhandlungsablauf der Mediation prägen und bestimmen. Eine solche Tätigkeit könnte weniger belastend sein und gegebenenfalls auch der Motivation der Richter, Rechtsfrieden zwischen den Parteien zu schaffen, entsprechen.

Allerdings ist dabei auch zu beachten, dass zusätzliche (Transaktions-)Kosten ${ }^{322}$ für den Richtermediator entstehen, da eine Zusatzausbildung erforderlich ist, um die notwendigen Fähigkeiten für die Mediatorentätigkeit zu erwerben. Wird langfristig aber der Erwerb von den für die Mediation erforderlichen Kenntnissen und Fähigkeiten im Ausbildungskanon etabliert, so entstehen keine zusätzlichen Transaktionskosten im Vergleich zur normalen Richtertätigkeit.

Darüber hinaus hat die Mediatorenausbildung auch positive Auswirkungen auf die Tätigkeit als gesetzlicher Richter. So werden durch die Ausbildung die Kommunikations- und Verhandlungsfähigkeit des Richters positiv beeinflusst und ihm Techniken vermittelt, die er auch im Rahmen von Vergleichsgesprächen im Prozess einsetzen kann.

\section{(2) Ausgestaltung der Richtermediatorentätigkeit}

Während des Modellversuchs besteht für die Richtermediatoren keine dem Deputatsschüssel vergleichbare Verpflichtung, eine bestimmte Anzahl von Mediationen durchzuführen. Somit könnte die Gefahr bestehen, durch die Tätigkeit als Richtermediator die persönliche Arbeitszeit zu verkürzen. Derzeit bestehen daher keine direkten Anreize, die Tätigkeit besonders leistungsorientiert auszurichten.

Auch wenn derzeit keine Anzahl an durchzuführenden Mediationen vorgegeben ist, so ist dennoch davon auszugehen, dass im Rahmen des Modellversuches ein Anreiz besteht, möglichst erfolgreich tätig zu sein und viele Mediationsverfahren durchzuführen. ${ }^{323}$ Der Erfolg des Richtermediators ist somit für das öffentliche Bild der Justiz und die Reputation der Richterschaft im Allgemeinen (s.o.) aus-

322 Der Begriff der Transaktionskosten ist grundlegend für die Neue Institutionenökonomik und ist weit zu verstehen. Transaktionskosten fallen bei der Errichtung, der Änderung und der Nutzung von Institutionen aller Art an. Umfassend zu diesem Begriff siehe Richter/Furubotn, S. 12 f., 55 ff., Eidenmüller, S. 97 ff.; Martiensen, S. 271 ff.; grundlegend zum Transaktionskostenansatz siehe Coase, The Problem of Social Cost, Economica 1937, 386 ff.

${ }^{323}$ Die Unterschiede in den „Erfolgszahlen“ zwischen den einzelnen Projektgerichten, insbesondere zum LG Göttingen lassen sich wohl vor allem auf die Unterschiede in den Abgabeverfahren an die Richtermediatoren erklären. Für eine Analyse über die Vorteilhaftigkeit des jeweiligen Verfahrens bleiben die Ergebnisse der empirischen Begleitforschung abzuwarten. 
schlaggebend und später auch für die Entscheidung, ob gerichtsnahe Mediation dauerhaft in das Angebot der Justiz aufgenommen werden soll. 324

227 Ist die Gerichtsnahe Mediation in das Leistungsangebot der Justiz aufgenommen, so könnte dieser Anreiz aber abgeschwächt werden. Daher ist auch bei einer langfristigen Installation der Gerichtsnahen Mediation an einen Deputatsschlüssel für die Richtermediatorentätigkeit zu denken, wobei jedoch das Problem berücksichtigt werden muss, dass das Mediationsergebnis bei der Gerichtsnahen Mediation von den Parteien und ihrem Verhalten stärker als im normalen Prozess abhängig ist. Zudem ist der Erfolg einer Mediation, ein Abschluss einer Mediationsvereinbarung, kein Nachweis einer guten Leistung des Richtermediators, sondern lediglich ein Indiz. Anders als ein Urteil ist die Richtermediatorentätigkeit nicht später überprüfbar. Umgekehrt gilt, dass das Scheitern der Mediationsverhandlungen nicht unbedingt auf schlechtes Verhalten des Richtermediators zurückzuführen ist.

228 Einen Lösungsansatz für das eben beschriebene Problem bietet der Ansatz, der am Landgericht Göttingen erprobt wird und der statt eines Deputatsschlüssels für Richtermediatoren eine Selbstbindung der Richtermediatoren vorsieht: ${ }^{325}$ Höhere Fallzuweisungen an die Kammern sollen zunächst einen stärkeren Anreiz zur Abgabe von Fällen an den Richtermediator geben. Um das eben beschriebene Problem zu entschärfen bzw. einen Ausgleich zwischen den Interessen zu finden, haben die Göttinger Richtermediatoren, die zunächst aus den Kammern abgezogen und mit ihrem für die gerichtsinterne Mediation notwendigen Arbeitsanteil in den Kammern entlastet wurden, in einer Selbstbindung eine bestimmte Anzahl monatlicher Mediationstermine und auch Erledigungen garantiert. Durch die hohen Erledigungszahlen tritt dann letztlich ein Entlastungseffekt zugunsten der richterlich tätigen Kollegen ein, der wiederum die Abgabebereitschaft der Kollegen aufgrund der positiven Erfahrungen stärkt. Voraussetzung für den Erfolg eines solchen Verfahrens ist natürlich auch hier eine positive Haltung aller am Gericht tätigen Personengruppen gegenüber der gerichtsinternen Mediation.

229 Eine Selbstbindung kann gegenüber einem verpflichtenden Pensenschlüssel stärker motivierend wirken. Außerdem werden, um diese zugesicherte Selbstbindung auch einhalten zu können, in Absprache mit den Parteien Mediationsverhandlun-

324 Trotz gleicher Voraussetzungen, also Freistellung der Richtermediatoren für die Richtermediatorentätigkeit, bestehen an den einzelnen Projektgerichten in der Rechtstatsächlichkeit Unterschiede in den mediierten Fällen. Dabei ist zunächst zu beachten, dass hohe Fallzahlen kein Ausdruck besonders hoher Qualität der Mediatorentätigkeit sind. Gleichwohl sind die Ursachen für diese Unterschiede im Rahmen der empirischen Erhebung zu vertiefen.

325 Hierzu Götzv. Olenhusen, ZKM 2004, 104, 105 f. 
gen in Göttingen aufgrund von Erfahrungswerten auf einen Termin von etwa 2 bis 2,5 Stunden begrenzt. Das Zusammenspiel von Selbstbindung und einer solchen Vereinbarung mit den Parteien ergibt einen sehr hohen Anreiz für die Richtermediatoren, in effizienter Weise auf eine Einigung der Parteien hinzuwirken.

Nach den bisherigen Erfahrungen aus dem Projekt erscheint dies als ein gangbares Modell, das daher empfohlen werden kann.

\section{dd) Gestaltung des Arbeitsalltags}

Staatliche Gerichte als Anbieter von Dienstleistungen zur Konfliktbehandlung, die sich bisher auch einer Monopolstellung für die Konfliktbehandlung sicher waren, sehen sich zunehmend durch die Konkurrenz außergerichtlicher Schlichtungsverfahren, vor allem durch die Mediation, einem bisher nicht gekannten Veränderungsdruck ausgesetzt. Konflikte, die zuvor zu ihnen kamen und auch zu ihrem Prestige beitrugen, wie etwa bedeutsame wirtschaftsrechtliche Konflikte, wandern zur ADR ab. ${ }^{326}$ Das Angebot der gerichtsnahen Mediation versucht nun, solche Fälle wieder an das Gericht zu ziehen. Wird das Angebot der gerichtsnahen Mediation auch gezielt von Parteien und Rechtsanwälten nachgefragt, so könnte eine Tätigkeit als Richtermediator daher auch eine Möglichkeit darstellen, interessante(re) und auch prestigeträchtigere Fälle verhandeln zu können.

\section{ee) Zufriedenheit mit der beruflichen Tätigkeit}

Ferner kann für den Richtermediator ein Anreiz bestehen, anstatt des „Kampfes“ im Prozess im Rahmen der Mediation Kooperation zwischen den Parteien zu fördern und so - abhängig von der persönlichen (psychischen) Belastbarkeit - das eigene Wohlbefinden und die Zufriedenheit mit der eigenen Vermittlungstätigkeit zu steigern.

Ein Richter ist zudem als imperfekter Entscheider zu betrachten, ${ }^{327}$ der in einigen Fällen Entscheidungen trotz unvollständiger Information treffen muss, eventuell auch anhand der Beweislage gegen seine innere Überzeugung. Dieses Problem der unvollständigen Information wird mit Hilfe der Mediationsverhandlungen durch die konsensuale Streitbeilegung durch die Parteien gelöst. Somit besteht ein Anreiz, imperfekte Entscheidungen durch die gerichtsnahe Mediation zu vermeiden.

\footnotetext{
326 Gottwald, AnwBL 2000, 265, 273.

327 Vgl. Schmidtchen/Kirstein, in: Schmidtchen/Werth, Effizienz als Rechtsprinzip, S. 207, $209 \mathrm{ff}$.
} 


\section{ff) Beförderungschancen und Verbesserung der Berufschancen}

234 Ein Anreiz für die Tätigkeit als Richtermediator kann auch in der Verbesserung der Beförderungschancen liegen. ${ }^{328}$ In jedem Fall wird durch die Tätigkeit als Richtermediator der Erwerb weiterer Qualifikationen unterstützt. Unter Umständen kann dadurch bei einem eventuellen Wechsel von der Tätigkeit zur Justiz hin zu einer privatwirtschaftlichen Tätigkeit auch das Einkommen gesteigert werden.

\section{gg) Ausblick}

235 Nach Ablauf des Modellversuches und einer eventuellen Entscheidung, gerichtsnahe Mediation auch weiterhin durch die Justiz anzubieten, ist langfristig an die Einführung eines der normalen Richterstelle vergleichbaren Deputatsschlüssels zu denken, um den Anreiz zur bloßen Erhöhung der persönlichen Freizeit durch die Tätigkeit als Richtermediator zu begrenzen (und eine „Flucht in die Tätigkeit als Richtermediator" zu vermeiden) und stärkere Anreize zur leistungsorientierten Ausgestaltung der Tätigkeit als Richtermediators zu setzen.

236 Statt der Einführung eines Deputatsschlüssels ist auch an eine Selbstbindung der Richtermediatoren ähnlich dem am Landgericht Göttingen praktizierten Modell zu denken. Der Vorteil besteht hier in dem Element der Freiwilligkeit für die Tätigkeit als Richtermediator.

237 Um die Qualität der Richtermediatorentätigkeit langfristig zu sichern und auch strategisches Verhalten einzelner Richtermediatoren zu vermeiden, ist an Überprüfungen (Bewertung durch die Parteien; Evaluation), Supervision und regelmäBige Weiterbildungen zu denken. ${ }^{329}$

238 Soll langfristig das Angebot gerichtsnaher Mediation mit sonstigen außergerichtlichen Konfliktangeboten konkurrieren, so sollte die Justiz auf dem Markt für Konfliktlösungsdienstleistungen ein positives Signal zur Gewährleistung bestimmter Qualitätsstandards der Tätigkeit der Richtermediatoren setzen. Dies kann durch

328 Cohen, 7 Journal of Law, Economics, and Organization 183, 184 (1991); Schmidtchen/Weth, in: Schmidtchen/Weth, Effizienz als Rechtsprinzip, S. 237, 243.

329 Art. 4 des Entwurfes der europäischen Mediationsrichtlinie fordert von den Mitgliedsstaaten, die Qualität der Mediationsanbieter durch Kontroll- und Fördermaßnahmen sicherzustellen, wovon explizit auch die Förderung freiwilliger Verhaltenskodizes erfasst wird, abgedruckt in: ZKM 2004, $149 \mathrm{f}$. 
eine Zertifizierung der Richtermediatorentätigkeit oder eine freiwillige Selbstbindung an den europäischen Verhaltenskodex für Mediatoren ${ }^{330}$ erreicht werden.

\section{Anreizstrukturen Anwälte}

Anwälte fungieren als Filter für die Nachfrage nach Gerichtsleistungen, ${ }^{331}$ somit auch für die Nachfrage nach gerichtsnaher Mediation. In der Regel werden die Parteien gemeinsam mit ihren Anwälten entscheiden, sich auf ein Mediationsverfahren einzulassen, bzw. auf den Rat ihrer Anwälte vertrauen, ob gerichtsnahe Mediation in ihrem Fall eine sinnvolle Alternative zum streitigen Verfahren darstellt. ${ }^{332}$ Den Anwälten kommt daher eine Schlüsselrolle im Hinblick darauf zu, ob sich die Partei für die gerichtsnahe Mediation entscheidet. Im Folgenden sollen daher die Rahmenbedingungen und Anreize untersucht werden, die die Entscheidung des Anwalts, seinem Mandanten eine gerichtsnahe Mediation zu empfehlen oder davon abzuraten, steuern.

\section{a) Principal-Agent-Problematik zwischen Anwalt und Mandant}

\section{aa) Das Problem}

Rät der Anwalt seinem Mandanten von einer gerichtsnahen Mediation ab, so ist dem Mandanten eine Bewertung dieses Urteils in der Regel nicht möglich, da ihm das notwendige juristische Wissen zumeist fehlen wird. Anwälte bieten eine Leistung an, die als Vertrauensgut zu beschreiben ist: Der Mandant kann mangels eigener rechtlicher Kenntnisse die Qualität der Leistung des Anwalts und die Richtigkeit seiner Aussagen nicht beurteilen und muss stattdessen auf die Aussagen des Anwalts vertrauen. Aufgrund dieser asymmetrischen Informationsverteilung zulasten der Mandanten besteht ein sog. Principal-Agent-Problem ${ }^{333}$ zwi-

330 In der englischen Originalfassung abgedruckt in ZKM 2004, 148 f., eine deutsche Übersetzung findet sich im mediations-report 8/2004, S. 2 f.

331 Schmidtchen/Weth, in Schmidtchen/Weth, Effizienz als Rechtsprinzip, S. 237, 243; zur Bedeutung der Anwaltschaft für die Verbreitung außergerichtlicher Streitbeilegung siehe Hoffmann-Riem, ZRP 1997, 190, 197; Ponschab, in: Breidenbach/Henssler, Mediation für Juristen, S. 93, 98; Wasilewski, Streitverhütung durch Rechtsanwälte.

332 Zu den Faktoren, die die Häufigkeit gütlicher Streitbeilegung bei Rechtsanwälten beeinflussen siehe auch die Untersuchungen von Berg-van der Wijk, in: Blankenburg/Gottwald/Strempel, Alternativen zur Justiz - Berichte, Analysen, Perspektiven, S. 65 ff. und Wasilewski, Streitverhütung durch Rechtsanwälte.

333 Ein Principal-Agent-Problem kann dann zwischen zwei Rechtssubjekten entstehen, wenn der Prinzipal (hier der Mandant) Aufgaben inklusive einem gewissen Entscheidungsspielraum an einen Agenten (hier den Anwalt) delegiert. Durch geeignete Anreize muss sichergestellt werden 
schen Mandant und Anwalt. ${ }^{334}$ Aus dieser ungleichen Informationsverteilung und fehlenden oder nur eingeschränkten Kontrollierbarkeit der Leistung des Anwalts durch den Mandanten resultieren Verhaltensspielräume für den Anwalt, die dieser zu seinem Vorteil ausnutzen kann. Es besteht dann die Gefahr, dass der Anwalt versucht, vorrangig seinen eigenen Nutzen und Vorteil und nicht den des Mandanten zu maximieren. ${ }^{335}$ Durch ein solches Verhalten entstehen sog. „agency costs" für den Mandanten. 336 Im vorliegenden Fall der gerichtsnahen Mediation könnten die Anwälte einen Einkommensverlust befürchten ${ }^{337}$ oder aufgrund sonstiger eigener, für den Mandanten nicht offensichtlichen Interessen versuchen, ihren Mandanten von der Durchführung der gerichtsnahen Mediation abzuraten. Auch ist zu bedenken, dass eine anwaltliche Fortbildung in Mediation, Konfliktmanagement und -lösung oder ähnlichen Gebieten noch nicht zum Standard

(optimalerweise bereits vor Vertragsschluss), dass der Agent eine eventuell bestehende Informationsasymmetrien nicht zu seinen Gunsten ausnutzt.

Der Agent beeinflusst bei der Erfüllung der ihm obliegenden Aufgaben nicht nur seinen eigenen Nutzen, sondern auch den des Prinzipals. Dabei besteht der Nutzen des Prinzipals zumeist in der Erzielung eines möglichst hohen Gewinns (vorliegend ist die Nutzenfunktion des Mandanten aber sowohl von materiellen als auch von immateriellen Faktoren geprägt, siehe unten Rn. 265 ff.), während der Nutzen des Agenten sich sowohl positiv aus der Höhe der Entlohnung als auch negativ der Höhe des Arbeitsaufwands zusammensetzt.) Aufgrund unterschiedlicher Ziele und Nutzenfunktionen von Prinzipal und Agent entstehen Interessenkonflikte und die Gefahr, dass der Agent diese Situation zu opportunistischem Verhalten ausnutzt. Diese Gefahr würde dann nicht bestehen, wenn sowohl Agent als auch Prinzipal vollständig informiert wären, dann könnte der Prinzipal den Agenten perfekt überwachen und gegebenenfalls opportunistisches Verhalten sanktionieren. In der Realität besteht aber zumeist ein Informationsgefälle. Zumeist weist der Agent die größere Sachnähe und Sachkenntnis als der Prinzipal auf, was ja auch gerade die Delegation der Aufgabe begründet, was dann der Agent auszunutzen versucht. Die Tätigkeit des Agenten kann daher vom Prinzipal aufgrund der fehlenden Information oft nicht unmittelbar beobachtet werden. Um die Gefahr opportunistisches Verhalten des Agenten zu vermeiden, muss der Prinzipal versuchen, geeignete Anreiz- und Informationsmechanismen zu installieren, um die Informationsasymmetrie abzumildern und seinen Nutzenverlust zu vermeiden.

Opportunistisches Verhalten des Agenten verursacht sog. Agency-Costs. Darunter fallen zum einen die Kosten zur Installation von geeigneten Anreiz- und Überwachungsmechanismen und zum anderen gerade der (noch trotz Überwachungsmechanismen noch verbleibende) Nutzenverlust durch (Residual-) Opportunismus.

Allg. zur Principal-Agent-Problematik vgl. Richter/Furubotn, S. 173 ff.; Janson, S. 80, jeweils m.w.N.

334 Watts, Bargaining through an Expert Attorney, 10 Journal of Law, Economics and Organization 168, 169 (1994); Wein, Recht durch Rechtsanwälte?, S. 54 ff.; Gilson/Mnookin, 94 Columbia Law Review 509, 511 f. (1994).

335 Bernstein, 141 University of Pennsylvania Law Review 2169, 2195 (1993).

336 Schmidtchen/Weth, in Schmidtchen/Weth, Effizienz als Rechtsprinzip, S. 237, 243.

337 Auch wenn durch die Einführung des RVG kein Gebührenverlust für die Anwälte besteht, ist damit keine Aussage über die tatsächliche Interessenlage der Anwälte getroffen. Diese Faktoren können vielfältig auch von nichtmonetärer Art sein. 
gehört und daher eventuell auch der Anwalt nicht in der Lage ist, die Mediationseignung oder die „Konfliktreife“ einzuschätzen. Der Rat des Anwalts muss daher - sowohl wegen der Gefahr opportunistischen Verhaltens als auch der bloßen Gefahr der fehlenden Sachkenntnis - nicht immer eine angemessene Lösung des Konfliktes darstellen.

Der Mandant muss mangels eigener rechtlicher Kenntnisse dem juristischen Rat seines Anwalts, seiner Einschätzung der Prozessaussichten und der Mediationseignung vertrauen. Daher kann die Partei auch nicht die Richtigkeit des Urteils des Anwalts einschätzen, wenn dieser von einem Mediationsverfahren abrät und stattdessen zur weiteren Verfolgung des Prozesses rät. So besteht z.B. die Gefahr, dass der Anwalt seinem Mandanten nur die Nachteile und nicht die Vorteile eines Mediationsverfahrens erläutert. Dabei kann der Mandant nicht kontrollieren, ob der Anwalt nur seinen eigenen Vorteil verfolgt oder auch in seinem Interesse handelt.

Auf die möglichen Verhaltensanreize für die Anwälte muss daher besonderes Augenmerk gelegt und überlegt werden, ob seine Interessen mit denen des Mandanten an einer angemessenen Konfliktlösung gleich laufen bzw. wie Anreize gesetzt werden könnten, um einen solchen Interessengleichlauf zu unterstützen. In diesem Zusammenhang sind daher zunächst grundsätzliche Überlegungen anzustellen, wie das eben beschriebene Principal-Agent-Problem überwunden und letztlich das Verhältnis zwischen Anwalt und Mandant strukturell verbessert werden kann.

\section{bb) Ansätze zur Überwindung des Principal-Agent-Problems:}

Als Lösungsmöglichkeit dieses Principal-Agent-Problems zwischen Mandant und Anwalt bietet sich ein Reputationsmechanismus an. ${ }^{338}$ Ebenso wie es sich auf die Reputation eines Anwalts langfristig negativ auswirken wird, wenn dieser seinen Mandanten immer und unverzüglich zum Klagen rät, nur weil er dadurch auf eine Erhöhung seiner eigenen Einnahmen hofft, reduziert auch ein kategorisches Abraten des Anwaltes von der gerichtsnahen Mediation ungeachtet der Mandanteninteressen und seiner besonderen Situation, z.B. einer persönlichen langjährigen Geschäftsbeziehung des Mandanten zur beklagten Partei, das öffentliche Ansehen dieses Anwalts. Sinken infolge einer schlechten Reputation die Mandantenzahlen,

338 Wein, Recht durch Rechtsanwälte?, S. 104 ff. Kobayashi/Parker, Civil Procedure: General, in: Bouckaert/ de Geest: Encyclopedia of Law and Economics (http://encyclo.findlaw.com/), 1, 13; Gilson/Mnookin, 94 Columbia Law Review 509, 513 (1994); Smith/Cox, 14 Journal of Legal Studies 167, 169 ff. (1985); Miller, 16 Journal of Legal Studies 189, 210 (1987). 
wird so ein allein eigennutzenmaxmierendes Verhalten des Anwalts sanktioniert: Nicht nur bestehende Vertrauensverhältnisse zu Mandanten können so geschädigt bzw. zerstört werden, sondern es würde auch aufgrund einer schlechten Reputation schwierig sein, neue Mandanten zu gewinnen.

244 Voraussetzung für die Wirksamkeit des Kontrollmechanismus der Reputation ist allerdings, dass die Öffentlichkeit über das Angebot der gerichtsnahen Mediation informiert und auch über Vor- und Nachteile umfangreich aufgeklärt ist. Daher ist es in jedem Fall erforderlich, dass nach Abgabe des Falles vom Fallmanager an den Richtermediator dieser auch Kontakt direkt mit den Parteien aufnimmt und sie über Chancen und Risiken der gerichtsnahen Mediation aufklärt. So wird das Problem der asymmetrischen Informationsverteilung und der fehlenden Einschätzbarkeit des Anwaltsverhaltens seitens der Parteien reduziert.

245 Auch können Gütesiegel, Zertifizierungen oder Akkreditierungen als Signale für die Mandanten wirken, die zeigen, dass der Anwalt Kenntnisse im Bereich Konfliktmanagement besitzt, und so stärkeres Vertrauen der Mandanten in das Urteil erzeugen. ${ }^{339}$ Gleichzeitig vermögen auch diese Gütesiegel die Qualität der Einschätzung des Anwalts zu erhöhen und eine angemessene Lösung des Konflikts unterstützen.

\section{b) Gebührenordnung}

246 Für die Anwälte bestehen zunächst durch die Gebührenordnung finanzielle Anreize, überhaupt tätig zu werden. Fraglich ist daher, ob durch die gesetzlichen Gebühren für den Anwalt auch ein Anreiz bestehen kann, seinen Mandanten die gerichtsnahe Mediation zu empfehlen.

\section{aa) Gerichtsnahe Mediation im Zivilprozess}

247 Wie bereits ausführlich dargelegt ${ }^{340}$ kann der Rechtsanwalt im Zivilprozess nach dem neuen Rechtsanwaltsvergütungsgesetz (RVG) für seine in der Mediation begleitend beratende Tätigkeit nun auch eine Vergütung in Form einer Terminsgebühr verlangen, was ihm nach der alten Rechtslage, also unter Geltung der BRAGO, nicht möglich war. Darüber hinaus stehen ihm, wie schon bisher, eine Verfahrensgebühr für die Einleitung des Verfahrens und bei Erfolg der Mediation eine Einigungsgebühr zu.

\footnotetext{
339 Siehe hierzu auch unten Rn. 254.

340 Siehe hierzu oben Rn. 139.
} 
Daher bleibt festzuhalten, dass der Anwalt im Hinblick auf die gesetzlichen Gebühren nach dem RVG nunmehr keine Gebührenverluste im Vergleich zu seiner Tätigkeit in einem herkömmlichen Gerichtsverfahren befürchten muss, wenn er seinem Mandanten die Inanspruchnahme des gerichtlichen Mediationsangebotes empfiehlt und die Partei in der Mediation begleitend berät.

Durch die nunmehr ausdrückliche Regelung des Entstehens der vollen Terminsgebühr auch bei Verhandlungen über nicht zum Streitgegenstand des anhängigen Gerichtsverfahrens gehörende Gegenstände in Höhe von 1,2 aus dem Wert der nicht rechtshängigen Ansprüche ${ }^{341}$ besteht zudem für den Anwalt ein positiver Anreiz, seinem Mandanten die Gerichtsnahe Mediation zu empfehlen, wenn ersichtlich ist, dass in dem Konflikt auch nicht rechtshängige Ansprüche mitverhandelt werden könnten.

\section{bb) Gerichtsnahe Mediation im verwaltungsgerichtlichen Verfahren}

Auch im verwaltungsgerichtlichen Verfahren erleidet der Anwalt durch die Empfehlung der Gerichtsnahen Mediation keinen Gebührenverlust, der einen negativen Anreiz darstellen könnte. ${ }^{342}$

\section{cc) Gerichtsnahe Mediation im sozialgerichtlichen Verfahren}

Ebenso wie im Zivilprozess wurde auch im sozialgerichtlichen Verfahren eine Vergleichs- und Erledigungsgebühr neu eingeführt. Im Vergleich zum Zivilprozess ist bei dieser Vergleichsgebühr zu beachten, dass sich diese im Vergleich zur Ausgangshöhe verringert, wenn über den sozialrechtlichen Gegenstand ein gerichtliches Verfahren anhängig ist. Darüber hinaus ergeben sich keine Unterschiede zum Zivilprozess, so dass das dort gesagte entsprechend gilt. Neben einer Verfahrensgebühr sieht das RVG auch eine Terminsgebühr vor. Folglich sind auch im sozialgerichtlichen Verfahren keine negativen Anreize im Vergleich zu einem normalen Prozess gegeben. Durch die Einführung dieser Vergleichs- und Erledigungsgebühr wurde bei der Mediation vielmehr ein positiver Anreiz gesetzt. ${ }^{343}$

\section{dd) Ergebnis}

In keinem der drei untersuchten Verfahren ergeben sich für den Anwalt negative Anreize aufgrund einer geringeren Vergütung; bei den sozialgerichtlichen Verfah-

\footnotetext{
341 Siehe hierzu oben Rn. 139.

342 Siehe hierzu oben Rn. 146.

343 Siehe hierzu oben Rn. 148.
} 
ren ergeben sich sogar positive Anreize. Folglich stellt die gerichtsnahe Mediation unter diesem Aspekt eine ebenbürtige, für die sozialgerichtlichen Verfahren darüber hinaus eine vorzugswürdige, Alternative dar.

\section{c) Transaktionskostenersparnis}

253 Durch die Gerichtsnahe Mediation könnte für den Anwalt und für sein gesamtes Anwaltsbüro noch eine Ersparnis an eigenem Arbeitsaufwand und Arbeitszeit verglichen mit einem normalen Gerichtsverfahren bestehen. ${ }^{344}$ Die Möglichkeit, eigenen Arbeitsaufwand und eigene Arbeitszeit zu sparen, kann auch bei der beschriebenen Kostenneutralität der gerichtsnahen Mediation für den Anwalt gegenüber dem streitigen Verfahren einen positiven Anreiz für den Anwalt setzen, seinem Mandanten die gerichtsnahe Mediation zu empfehlen, wenn er einen eventuellen Einkommensverlust durch eine Ersparnis an eigenem Arbeitsaufwand und dem seines gesamten Anwaltsbüros ausgleichen kann.

\section{d) Berufsständische Regulierungen}

254 Auch berufsständische Regulierungen können - wie bereits dargelegt ${ }^{345}$ - dazu beitragen, das zwischen Anwalt und Mandant bestehende Principal-AgentProblem zu lösen bzw. die Kosten, die durch die Ausnutzung der oben dargelegten Verhaltensspielräume entstehen (,agency costs"), zu reduzieren. 346 Allerdings existieren derzeit keine Verhaltenspflichten, die auf ein Fördern der Mediation abzielen. De lege ferenda wäre daher zur Kontrolle des anwaltlichen Verhaltens an eine Einführung von berufsständischen Regulierungen zu denken, die für die Anwälte Anreize zur Förderung der (gerichtsnahen) Mediation setzen.

255 Hier ist zunächst an Fortbildungen im Konfliktmanagement zu denken, um die Fähigkeiten der Anwälte bei der Einschätzung und Lösung von Konflikten, von Mediationseignung und Konfliktreife zu fördern und so dem Mandanten einen angemessenen Lösungsvorschlag für seinen Konflikt unterbreiten zu können. Darüber hinaus sollten diese Fortbildungen auch für den Mandanten erkennbar sein, damit dieser den Rat des Anwalts auch einschätzen kann. Hier sind Gütesie-

\footnotetext{
344 Auch Huther, ZKM 2004, 247, 250 betont den Aspekt der Zeitersparnis für die Anwälte; Lande, UCLA Law Review 69 (2000), 69, 120.

345 Siehe Rn. 243.

346 Miller, 16 Journal of Legal Studies 189, 209 f. (1987); Kobayashi/Parker, Civil Procedure: General, in: Bouckaert/ de Geest: Encyclopedia of Law and Economics (http://encyclo.findlaw.com/), 1, 13.; Schmidtchen/Weth, in: Schmidtchen/Weth, Effizienz als Rechtsprinzip, S. 237, 243.
} 
gel, Zertifizierungen, Akkreditierungen oder ähnliche als Signal wirkende Instrumente denkbar.

\section{e) Reputation}

\section{aa) Reputationsgewinn}

Die eigene Reputation könnte für den Anwalt nicht nur einen Kontrollmechanismus, sondern zugleich einen positiven Anreiz für das eigene Verhalten darstellen: Ein Anreiz zur Förderung der (gerichtsnahen) Mediation könnte für die Anwälte nämlich dann bestehen, wenn die Mandanten nach erfolgreichem Abschluss einer Mediation tatsächlich zufriedener mit dem ausgehandelten Ergebnis und dem Verfahren wären als mit einem normalen streitigen Verfahren. Der Mandant würde sich dann sehr gut von seinem Anwalt beraten wissen und zum einen sich selbst bei weiteren Konflikten und Rechtsstreitigkeiten erneut an diesen Anwalt wenden, zum anderen aber auch in seinem eigenen Umfeld und Bekanntenkreis diesen Anwalt weiterempfehlen. Die Reputation des Anwalts könnte steigen; er könnte neue Mandanten, somit zusätzliche Fälle gewinnen und dadurch letztlich auch einen Einkommensgewinn erzielen. ${ }^{347}$

\section{bb) Reputationsverlust}

Mit der Möglichkeit eines Reputationsgewinnes geht aber zugleich die Gefahr eines Reputationsverlustes im Einzelfall einher. Empfiehlt z.B. der Anwalt des Klägers diesem zunächst aufgrund hoher Erfolgsaussichten eine Klageeinreichung, so kann es unter Umständen von dem Mandanten als ein Zeichen von fehlenden rechtlichen Fähigkeiten, fehlender Glaubwürdigkeit oder Schwäche aufgefasst werden, wenn er dann zum Eintritt in die gerichtsnahe Mediation rät. Für den Anwalt des Beklagten kann ein Zuraten zur Mediation unter Umständen ebenfalls als Zeichen fehlender Kompetenz bewertet werden. Der Anwalt könnte daher zögern, seinem Mandanten zu einer gerichtsnahen Mediation zu raten.

Dieses Problem stellt sich allerdings in den Fällen nicht, wenn Anwalt und Mandant die Klage nur eingereicht haben, um das Angebot der gerichtsnahen Mediation wahrnehmen zu können. 


\section{f) Arbeitsmarktbedingungen und Gewinn von zusätzlicher Ausbil- dung und Erfahrung}

259 Ein weiterer Anreiz zur Unterstützung der gerichtsnahen Mediation für den Anwalt könnte in den Arbeitsmarktbedingungen bestehen. Mediation hat sich in den vergangenen Jahren als attraktives Berufsfeld und Betätigungsmöglichkeit auch für Anwälte entwickelt. Gleichzeitig nimmt die Anzahl der Anwälte in Deutschland immer mehr zu, so dass eine Erweiterung des Leistungsangebots der Anwälte erforderlich ist. Durch die Teilnahme und Begleitung der Mandanten im Rahmen der gerichtsnahen Mediation könnten auch die Anwälte eigene Erfahrungen für eine Tätigkeit als Mediator sammeln und so ihre Chancen auf dem Arbeitsmarkt langfristig erhöhen.

\section{g) Fazit}

260 Die Änderung der Gebührenstruktur durch die Einführung des RVG hat die früher bestehende Problematik entschärft. Durch die neue Gebührenordnung werden negative finanzielle Anreize für den Anwalt durch die Empfehlung der Gerichtsnahen Mediation vermieden. Dadurch wurde auch die oben beschriebene Principal-Agent-Problematik zwischen Anwalt und Mandant entschärft.

261 Zur Förderung der gerichtsnahen Mediation und zur Entschärfung des PrincipalAgent-Problems ist zusätzlich auch an eine berufsständische Verhaltenspflicht für Anwälte zu denken, mit den Parteien die Alternative einer (gerichtsnahen) Mediation zu diskutieren und Möglichkeiten und Grenzen derselben auszuloten. ${ }^{348}$ Eine Selbstregulierung durch Gütesiegel, Zertifizierung oder Akkreditierung erscheint hier als wirkungsvolles Instrument.

262 Ob darüber hinaus aber auch ein deutlicher positiver Anreiz durch die Gebührenordnung für den Anwalt gesetzt werden sollte, kann an dieser Stelle noch nicht eindeutig bewertet werden. Insbesondere ist hier von Interesse, ob die Anwälte sich tatsächlich von der Gerichtsnahen Mediation eine eigene Zeitersparnis und eine höhere Parteizufriedenheit, die sich positiv auf ihre Reputation auswirken könnte, erhoffen und ob diese tatsächlich eintreten.

263 Besteht tatsächlich eine deutliche Zeitersparnis durch die Gerichtsnahe Mediation, so erscheint die Gerichtsnahe Mediation auch bei gleicher Gebührenhöhe für die Anwaltschaft als vorteilhaft. Hierbei gilt es zu bedenken, dass ein für die Anwaltschaft gesetzter positiver Anreiz durch die Gebührenordnung eine zusätzliche

348 Hager, Konflikt und Konsens, S. 105. 
Belastung des Mandanten mit höheren Anwaltskosten mit sich bringen würde, so dass der für die Anwaltschaft gesetzte positive Anreiz für die Mediation u.U. durch einen negativen Anreiz für die Konfliktparteien ausgeglichen werden könnte.

Das Risiko des Scheiterns der Mediation und des damit verbundene Arbeitsmehraufwands bei gleicher Gebührenhöhe muss nicht zwangsläufig den Anreiz für die Anwälte mindern. Zum einen verhindert dieses ein mögliches strategisches Verhalten des Anwalts, jedes Verfahren zur Arbeitsaufwandsreduzierung in die Mediation abzugeben, sondern schärft vielmehr den Blick auf den Konflikt und fördert Aus- und Weiterbildung im Konfliktmanagement zur Vertiefung der Kenntnisse im Erkennen der Konfliktreife. Zum anderen können selbst bei einer gescheiterten Mediation positive Auswirkungen auf den Prozess bestehen, die insbesondere zu einer Beschleunigung führen können, so dass der vermeintliche Arbeitsmehraufwand durch Arbeitsersparnisse im nachfolgenden Prozess wieder ausgeglichen werden kann.

\section{Anreizstrukturen Parteien}

Wurde eben die versteckte Motivation der Anwälte im Verhältnis zu ihren Mandanten untersucht, so soll nun untersucht werden, ob die gerichtsnahe Mediation für die Parteien wirklich vorteilhaft ist und welche Anreize für die Parteien bestehen, sich auf die gerichtsnahe Mediation selbst bei erfolgversprechenden Prozessaussichten einzulassen, d.h. insbesondere ein wahrscheinliches für den Kläger positives Urteil gegen einen in der Mediation ausgehandelten und protokollierten Prozessvergleich „einzutauschen“. Der Kläger bzw. Beklagte wird immer jene Alternative wählen, die seinen Nutzen maximiert. Allerdings beziehen die privaten Akteure nicht nur alle materiellen Kosten und Nutzen wie Gerichtskosten oder Schadenssummen, sondern auch ihre immateriellen Kosten und Nutzen - z.B. Ärger im Prozess, Wunsch nach Gerechtigkeit, die Beziehung zur anderen Partei, aber auch die Gefahr möglicher Rechtsverluste - in ihr Kalkül mit ein.

\section{a) Kostenersparnis}

\section{aa) Kosten für Gerichts- und Mediationsverfahren}

Zunächst soll geklärt werden, welche direkten Kosten den Parteien für die gerichtsnahe Mediation im Vergleich zum herkömmlichen streitigen Gerichtsverfahren entstehen, werden doch die Anreize der Parteien maßgeblich durch die Kos- 
tenrisiken bestimmt. ${ }^{349}$ Jedoch ist dabei zu beachten, dass wesentliche Faktoren für die Entscheidung der Parteien neben den Gerichts- und Anwaltsgebühren auch die gesetzlichen Regelungen zur Verteilung der Kostenlast und die Erfolgsaussichten im Prozess darstellen. Die tatsächliche Entscheidung ist dann durch ein komplexes Wechselspiel dieser Faktoren gekennzeichnet.

267 Dabei können die Kosten anwaltlicher Beratung als Kostenaufwand weitgehend ausgeklammert werden, da sie gleichermaßen sowohl im Vorfeld einer Mediation als auch im Vorfeld eines ,normalen“ Rechtsstreits entstehen können. ${ }^{350}$

\section{(1) Gerichts- und Mediationskosten nach geltendem Recht}

\section{(a) Zivilprozess}

268 Im Gegensatz zum streitigen Zivilprozess entsteht in der gerichtsnahen Mediation aufgrund des geschlossenen Vergleichs lediglich eine statt drei Gerichtsgebühren. Kosten für Zeugen, Sachverständige und sonstige gerichtliche Auslagen, sofern diese denn überhaupt anfallen, bestehen jedoch auch weiterhin. ${ }^{351}$

\section{(b) Verwaltungsgerichtsverfahren}

269 Auch im verwaltungsgerichtlichen Verfahren entstehen drei Gerichtsgebühren. Diese werden auf eine Gebührt ermäßigt, wenn das Verfahren durch Klagerücknahme, Anerkenntnis- oder Verzichtsurteil, Erledigungserklärung oder einen gerichtlichen Vergleich zum Abschluss gebracht wird. Folglich fällt im Rahmen einer erfolgreichen gerichtsnahen Mediation nur eine Gebühr an. ${ }^{352}$

\section{(c) Sozialgerichtsverfahren}

270 Im Sozialgerichtsverfahren ist zwischen privilegierten und nicht privilegierten Parteien zu unterscheiden. Für privilegierte Parteien ist das Gerichtsverfahren kostenfrei, was auch für die gerichtsnahe Mediation gilt. Ist an dem Rechtsstreit darüber hinaus eine nicht privilegierte Partei beteiligt, so entstehen für diese Kosten in Höhe einer Kostenpauschale in Höhe von 150 bis 300 Euro.

271 Ist keine der Parteien privilegiert, so entstehen, wie im Zivil- bzw. Verwaltungsgerichtsprozess auch, drei Gebühren, die sich bei einer Beendigung des Verfahrens

\footnotetext{
349 So auch Wagner, JZ 1998, 836, 840.

350 Ditges, IDR 2005, 74, 75/ 81.

351 Siehe hierzu Rn. 133.

352 Siehe hierzu Rn. 135.
} 
durch Klagerücknahme, Anerkenntnisurteil, Erledigungserklärung oder einen gerichtlichen Vergleich auf eine einfache Gebühr reduzieren. ${ }^{353}$

\section{(d) Ergebnis}

Aus der Reduzierung der Gerichtskosten der Parteien von drei Gebühren im streitigen Verfahren auf eine Gebühr im Wege der gerichtsnahen Mediation ergeben sich somit positive Anreize für die Beteiligten. Für die Zeit der Kostenfreiheit der gerichtsnahen Mediation ist im Hinblick auf die Gerichtskosten daher insgesamt im Vergleich zum herkömmlichen streitigen Verfahren, das mit einem Urteil abschließt, von einer Kostenersparnis der Parteien auszugehen ist, wenn sie sich für die Mediation entscheiden und das Mediationsverfahren erfolgreich ist. ${ }^{354}$ Scheitern die Mediationsverhandlungen, so entstehen zumindest keine höheren Kosten als im Falle eines herkömmlichen streitigen Gerichtsverfahrens.

\section{(2) Kostenregelung für die gerichtsnahe Mediation in der Zukunft}

Wettbewerbsrechtliche Bedenken zeigen bei einer langfristigen Aufnahme von Mediation in das Leistungsangebot der Justiz Handlungsbedarf auf. 355 Daher sind nachdrücklich eine Änderung der ZPO und die gesetzliche Normierung der gerichtlichen Mediation zu empfehlen. Eine Änderung der Gebührentabelle wäre dann nicht unbedingt erforderlich. Dass die Parteien vorgerichtliche Mediationsoder Streitschlichtungsangebote durch die gerichtsnahe Mediation substituieren, also Klage erheben, um das Angebot der gerichtsnahen Mediation ohne die Gefahr zusätzlicher Kosten durch das Scheitern einer vor- oder außergerichtlichen Mediation nutzen zu können, ist dadurch wohl kaum zu erwarten. Eine Förderung der vor- und außergerichtlichen Mediation könnte auch dergestalt erreicht werden, dass die Gerichtsgebühren im nachfolgenden streitigen Verfahren bei durchgeführter vorgerichtlicher Mediation teilweise erlassen werden könnten, um so auch Anreize für die Parteien zur Nutzung anderer Streitschlichtungsangebote zu setzen. Ist noch keine Klage erhoben, so ist davon auszugehen, dass Anwälte ihren Parteien nicht sofort den Weg zur gerichtsnahen Mediation raten werden, sondern die Inanspruchnahme einer außergerichtlichen Mediation empfehlen. Denkbar sind hier Kooperationen der Anwälte innerhalb der Rechtsanwaltskammer, in geeigneten Fällen - selbstverständlich unter der Gewährleistung gewisser Qualitätsstandards - Mitglieder dieser Kooperation für eine vorgerichtliche Mediation zu empfehlen.

\footnotetext{
353 Siehe hierzu Rn. 136.

354 Dazu Göthlich, IDR 2005, 68, 72.

355 Ausführlich hierzu oben Rn. 33.
} 
274 Sollte eine solche gesetzliche Normierung der gerichtlichen Mediation nicht erfolgen, so machen für die Zukunft die Vorgaben des Wettbewerbsrechts die Erhebung von Kosten für die Inanspruchnahme des gerichtlichen Mediationsangebotes unerlässlich.

275 Dabei könnten sich die Kosten für die gerichtsnahe Mediation vergleichbar den herkömmlichen Gerichtskosten an einer Gebührentabelle orientieren. In wettbewerbsrechtlicher Hinsicht wäre dies unbedenklich: Soweit bei geringen Streitwerten im Fall von inhaltlich und zeitlich überdurchschnittlich aufwändigen Mediationsverfahren vergleichsweise niedrige Gebühren berechnet würden, könnte dies durch einfacher gelagerte Mediationsverfahren mit hohen Streitwerten ausgeglichen werden. 356

276 Für die Konfliktparteien und private Mediationsanbieter transparenter wäre jedoch eine Kostenregelung, die sich nach dem konkreten Arbeitsaufwand des Richtermediators richtet und sich der Höhe nach an den durchschnittlichen Stundenhonoraren privater Mediationsanbieter ${ }^{357}$ orientiert. Ein solches Kostenmodell hätte den Vorteil, dass es einem direkten Vergleich mit den Honoraren privater Anbieter zugänglich wäre und eine wettbewerbsrechtlich problematische Konkurrenzsituation von vornherein vermieden werden könnte.

277 Nicht zu empfehlen ist es dagegen, bei der Regelung der Kosten für die gerichtsnahe Mediation auch an den konkreten gerichtlichen Arbeitsaufwand in Zusammenhang mit dem anhängigen Gerichtsverfahren anzuknüpfen. Mit einer solchen Regelung könnte zwar den durch die Einführung von gerichtlichen Mediationskosten gesetzten negativen Anreizen für die Parteien ein positiver Anreiz insoweit entgegen gesetzt werden, als in Fällen, in denen ein - erfolgreich durchgeführtes - Mediationsverfahren ohne vorheriges Tätigwerden des gesetzlich zuständigen Richters eingeleitet wurde und dieser in der Sache folglich keinen Arbeitsaufwand hatte, die Gerichtskosten über das ohnehin schon in Nr. 1211 Abs. 3 Anlage 1 GKG vorgesehene Maß hinaus reduziert werden könnten. Auch in wettbewerbsrechtlicher Hinsicht wäre eine solche, am konkreten Arbeitsaufwand angelehnte Gebührenstruktur für Gerichts- und Mediationskosten durchaus vertretbar. Doch sprechen Praktikabilitätsgesichtspunkte ebenso wie die Zielrichtung des Kostenrechtsmodernisierungsgesetzes, das Kostenrecht zu vereinfachen, ${ }^{358}$ eindeutig gegen eine solche Vorgehensweise: Da die Einleitung eines gerichtlichen

\footnotetext{
356 Volkmann, Mediation im Zivilprozess. Rechtliche Rahmenbedingungen für ein gerichtsinternes Mediationsangebot, S. 139 ff., auch zum folgenden.

357 Diese variieren zwischen 20,- und 400,- Euro, s. dazu Horst, ZKM 2004, 178, 180.

358 BT-Drucks. 15/1971, S. 1, 144.
} 
Mediationsverfahrens praktisch in jedem Stadium eines noch nicht zum Abschluss gelangten Prozesses erfolgen kann, wäre für jeden Fall individuell zu entscheiden, ob der durch die Mediation ersparte Arbeitsaufwand des gesetzlich zuständigen Richters eine weitere Gebührenreduktion rechtfertigt oder nicht.

Die Einführung von Gebühren für die gerichtsnahe Mediation kann starke Auswirkungen auf die Anreizstruktur der Parteien haben. Insbesondere ist bei der Ausgestaltung der Gebührenstruktur zu beachten, dass keine negativen Anreize gesetzt werden. Grenzen für die Gebührengestaltung werden von den Gebühren für die herkömmlichen Verfahren gesetzt. Für die Mediation sollten nicht mehr als drei Gebühren erhoben werden, da ansonsten kein Anreiz mehr besteht, sich gegen die Fortsetzung eines streitigen Verfahrens zu entscheiden, das mit Sicherheit eine Lösung des Konflikts bietet, während der Mediation auch immer die Gefahr des Scheiterns innewohnt.

\section{bb) Rechtsanwaltskosten}

Eine begleitende anwaltliche Beratung der Parteien während des Mediationsverfahrens ist vor dem Hintergrund des Rechtsberatungsgesetzes nicht erforderlich. ${ }^{359}$ Es kann jedoch empfehlenswert sein, dass sich die Parteien zusätzlich zu der vom Mediator zu leistenden Unterstützung auch in der Mediation von ihren jeweiligen Parteianwälten beraten lassen. Im Hinblick auf die den Parteien durch die Inanspruchnahme einer anwaltlichen Begleitung in der Mediation entstehenden Anwaltskosten kann auf die vorstehenden Ausführungen unter Rn. 139 verwiesen werden. Gebührenrechtlich macht es nach dem RVG nun keinen Unterschied mehr, ob die Parteien einen herkömmlichen Prozess mit anwaltlicher Vertretung führen oder an einer gerichtsnahen Mediation mit der Unterstützung ihrer Parteianwälte teilnehmen. Einen finanziellen Vorteil bietet die gerichtsnahe Mediation im Hinblick auf die Anwaltskosten damit nicht.

\section{cc) Verteilung der Kostenlast}

Auswirkungen auf die Entscheidung des Klägers, das streitige Verfahren fortzusetzen oder die gerichtsnahe Mediation zu wählen, haben auch die Regelungen zur Verteilung der Kostenlast.

\footnotetext{
${ }^{359}$ Siehe oben Rn. 22.
} 


\section{(1) Kosten für Gerichts- und Mediationsverfahren}

\section{(a) Zivilprozess}

\section{(aa) Scheitern der Mediationsverhandlungen und Fortführung des gerichtlichen Verfahrens}

281 Im Falle des Scheiterns der Mediationsverhandlungen und des Abschlusses des Verfahrens durch Urteil hat die unterliegende Partei gem. \91 ZPO in einem Zivilprozess die Kosten der obsiegenden Partei für die anwaltliche Begleitung in der Mediation und darüber hinaus auch mögliche künftig für das gerichtliche Mediationsangebot anfallende Gerichtskosten zu tragen. ${ }^{360}$ Allerdings wird hier langfristig wohl kein Unterschied zu der Wahrnehmung außergerichtlicher Mediationsangebote bestehen. Es wurde bereits für die Erstattung außergerichtlicher Kosten in einem obligatorischen Schlichtungsverfahren im Sinne von $\int 15$ a EGZPO entschieden, dass im Falle des Scheiterns die für das Schlichtungsverfahren aufzuwendenden Kosten als notwendige Vorbereitungskosten im Rahmen der Kostenerstattung nach $\int 91 \mathrm{ZPO}$ in einem nachfolgenden Klageverfahren grundsätzlich erstattungsfähig sind. ${ }^{361}$

\section{(bb) Einigung im Laufe der Mediationsverhandlungen und Protokol- lierung eines Prozessvergleichs}

282 Bei einem erfolgreichen Mediationsverfahren sind die Kosten der Parteien gem. \ 98 ZPO, keine anderweitigen Vereinbarungen vorausgesetzt, gegeneinander aufzuheben, wobei jedoch eine vertragliche Regelung den Regelfall darstellt. ${ }^{362}$

\section{(b) Verwaltungsgerichtsverfahren}

283 Wie im Zivilprozess so hat auch im Verwaltungsprozess die unterliegende Partei die Kosten des Rechtsstreits zu tragen. Bei einem Vergleich und fehlender Vereinbarung haben die Parteien die Kosten je zur Hälfte zu tragen. ${ }^{363}$

\footnotetext{
360 Siehe hierzu Rn. 150.

361 BayObLG ZKM 2004, 281.

362 Siehe hierzu Rn. 155.

363 Siehe hierzu Rn. 157.
} 


\section{(c) Sozialgerichtsverfahren}

Da das Gericht im Sozialgerichtsverfahren, bei dem eine privilegierte Person beteiligt ist, die Kostenentscheidung nach billigem Ermessen trifft, wobei es das Ergebnis des Rechtsstreits und den Sach- und Streitstand zu berücksichtigen hat, ergibt sich grundsätzlich nichts anderes als im Zivil- bzw. Verwaltungsprozess, so dass insbesondere die Rechtsanwaltsgebühren der nicht privilegierten Partei auferlegt werden können. ${ }^{364}$

In Konstellationen, in denen weder Kläger noch Beklagter privilegiert sind, finden gemäß \ 197 a Abs. 1 SGG die verwaltungsprozessrechtlichen Regelungen Anwendung, so dass das zum Verwaltungsgerichtsverfahren gesagte entsprechend gilt.

\section{(d) Ergebnis}

Da sich im Vergleich zu den Kostentragungsregeln eines streitigen Verfahrens keine Abweichungen bei einem Mediationsverfahren ergeben, sind hier keine negativen Anreize ersichtlich.

\section{(2) Immaterielle Kosten}

Ferner gilt für die Kostenerstattungspflicht des \91 ZPO ein materieller Kostenbegriff, so dass immaterielle Kosten wie z.B. die psychische Belastung eines Beklagten, der zu Unrecht in einen Rechtsstreit verwickelt wird, selbst bei Obsiegen im Prozess nicht ersetzt werden. Diese nicht ersetzten immateriellen Kosten senken den Erwartungswert für den Prozess ${ }^{365}$ und erhöhen damit auch die Bereitschaft, sich auf gerichtsnahe Mediation einzulassen.

\section{(3) Scheitern der gerichtsnahen Mediation}

Beide Parteien haben ferner die Gefahr des Scheiterns der Mediation zu berücksichtigen. Zusätzlich zu den eventuellen Kosten für eine anwaltliche Begleitung in der Mediation müssen beide Parteien in ihre Überlegungen die Verteilung der Kostenlast im wieder aufgenommenen streitigen Prozess gemäß \91 ff. ZPO einbeziehen.

\footnotetext{
364 Siehe hierzu, insbesondere zu den Besonderheiten des Sozialgerichtsprozesses Rn. 158.

365 Wein, Recht durch Rechtsanwälte?, 1995, S. 238 f.
} 


\section{(4) Kostenverteilungsregelungen und Erfolgsaussichten}

289 Die Auswirkungen der Regelungen zur Verteilung der Kostenlast auf die Entscheidung der Partei für oder gegen die gerichtsnahe Mediation sind von verschiedenen Faktoren abhängig, in erster Linie von der Risikoaversion der Parteien und den Erfolgsaussichten, die die beteiligten Parteien einem jeweiligen Obsiegen bzw. Unterliegen im Prozess beimessen. Ferner wirken sich auch immaterielle Kosten und Nutzen auf die Bildung des Erwartungswertes aus.

290 So hat ein Kläger, der seine Erfolgsaussichten der Klage als sehr aussichtsreich einschätzt, durch die Regelung des $₫ 91$ ZPO einen Anreiz, das streitige Verfahren fortzuführen, da er davon ausgeht, dass mit einer hohen Wahrscheinlichkeit der Beklagte gemäß \91 ZPO auch seine Kosten des Rechtsstreits mit übernimmt. Er geht also von einem hohen Erwartungswert der Klage aus.

291 Hingegen hat ein Kläger, der die Erfolgsaussichten seiner Klage und somit den Erwartungswert als gering einschätzt, gegebenenfalls einen Anreiz, sich auf eine gerichtsnahe Mediation einzulassen, da er dann nicht Gefahr läuft (das Scheitern der Mediation einmal ausgenommen), bei Unterliegen im Prozess gemäß \91 ZPO auch die Prozesskosten des Beklagten zu tragen.

292 Gleiches gilt für den Beklagten. Für ihn besteht dann eher ein Anreiz zur gerichtsnahen Mediation, wenn seine subjektive Wahrscheinlichkeit, den Prozess zu verlieren und die Kostenlast gemäß $\ 91$ ZPO zu tragen, sehr hoch ist. Die Kosten würden sich die Parteien dann in der Regel teilen.

293 Vice versa ist davon auszugehen, dass mit zunehmender subjektiver Erfolgswahrscheinlichkeit für den Beklagten kein finanzieller Anreiz durch die Gerichts-, Mediations- und Anwaltskosten für die gerichtsnahe Mediation besteht, da er es für wahrscheinlich hält, dass er keine Kosten der anderen Partei zu tragen hat.

\section{(5) Kostenanreize in Form von Kostensanktionen bereits im geltenden Recht?}

294 Ein Blick auf andere Rechtsordnungen zeigt, dass zusätzliche Anreize zur Inanspruchnahme des gerichtlichen Mediationsangebotes durch die Möglichkeit von Kostensanktionen nach dem Vorbild des englischen Prozessrechts geschaffen werden können: Mit der Reform des Zivilprozessrechts durch die am 26. April 1999 in Kraft getretenen Civil Procedure Rules (CPR 1998) ${ }^{366}$ wurde den engli-

\footnotetext{
366 Abrufbar unter: http://www.hmso.gov.uk/si/si1998/19983132.htm.
} 
schen Gerichten die Möglichkeit gegeben, einer Partei, die sich einem gerichtlich angeregten Versuch einer einvernehmlichen außergerichtlichen Konfliktbeilegung ungerechtfertigt widersetzt, gemäß Rule 44.3 (4) und (5) CPR 1998 die Kosten des Rechtsstreits ganz oder zum Teil auch dann aufzuerlegen, wenn sie in dem Rechtsstreit obsiegt. Zwar gilt auch im englischen Prozessrecht der Grundsatz, dass die unterliegende Partei die Kosten des Rechtsstreits zu tragen hat. ${ }^{367}$ Doch sollen mit dieser Regelung die durch die CPR 1998 vorgesehenen Bemühungen des Gerichts zur Förderung der einvernehmlichen außergerichtlichen Streitbeilegung $^{368}$ unterstützt werden. ${ }^{369}$

Die englischen Gerichte haben ihren Auftrag, den Parteien die Wahrnehmung außergerichtlicher Konfliktlösungsangebote nahezulegen, in die Tat umgesetzt $\mathrm{t}^{370}$ und auch von der Möglichkeit Gebrauch gemacht, eine unkooperative Partei gegebenenfalls durch Kostensanktionen zu bestrafen. ${ }^{371}$ Ansatzpunkt ist dabei die Ausgestaltung des englischen Prozesskostenrechts, welches die Auferlegung von Verfahrenskosten in das Ermessen des Gerichts stellt und so dem Gericht die Möglichkeit bietet, auch der obsiegenden Partei alle oder einen Teil der entstandenen Verfahrenskosten aufzuerlegen, sofern es dies unter Berücksichtung der Einzelfallumstände respektive des Parteiverhaltens als angemessen erachtet. ${ }^{372}$ Der englische Court of Appeal hat dazu, um den Zugang zu den staatlichen Gerichten nicht unverhältnismäßig einzuschränken und damit dem Anspruch auf ein faires, rechtsstaatliches Verfahren gerecht zu werden, 373 inzwischen einen Kriterienkatalog entwickelt, unter welchen Voraussetzungen eine Partei berechtigter Weise die Teilnahme an einer Mediation verweigern darf: Maßgebend sind die Art der Streitigkeit, die Erfolgsaussichten der siegreichen Partei im Prozess, die vorherige Ausschöpfung anderer Konfliktbeilegungsmethoden, die Kosten des Mediationsver-

\footnotetext{
367 Rule 44.3 (2) CPR 1998, abrufbar unter: http://www.hmso.gov.uk/si/si1998/19983132.htm.

368 Gemäß Rule 1.4 (2) e CPR 1998 soll das Gericht die Parteien in geeigneten Fällen zum Gebrauch außergerichtlicher Streitbeilegungsmethoden ermutigen.

369 Newmark, SchiedsVZ 2003, 23, 24.

370 Cable \& Wireless v. IBM UK Ltd. [2002] EWHC 2059 (Comm); Shiramayama Shokusan v. Danovo Ltd. [2003] EWHC 3006 (Ch.); zur Entwicklung des englischen Case Law im Hinblick auf die Neuregelungen der CPR 1998 siehe Newmark, SchiedsVZ 2003, 23, 25 ff.

371 Shiramayama Shokusan v. Danovo Ltd. [2003] EWHC 3006 (Ch.); ausführlich zu dieser Rechtsprechung $G$. Wagner, ZKM 2004, 100 ff.

372 Seit Inkrafttreten des CPR wurde dies zur Umsetzung der Rule 1.3 CPR genutzt, vgl. Dunnet v. Railtrack plc. [2002] 2 All ER 850; Hurst v. Leeming v. Leeming Defendant [2003] 1 Lloyds Rep 379; Halsey v. Milton Keyes General NHS Trust/Steel v. Joy and Halliday, [2004] All ER (D) 125 (May); jeweils kurz dargestellt bei Althammer, JZ 2006, 69, 70; Baumann, IDR 2005, 9, 11 ff.

373 Siehe dazu EGMR EUGRZ 1980, 667, 672 Nr. 49.
} 
fahrens, die Verzögerung des gerichtlichen Verfahrens durch die Zwischenschaltung der Mediation sowie die Erfolgsaussichten der Mediation. ${ }^{374}$ Die wesentlichen Ziele der Reform wurden erreicht: Die Anwendung der neuen CPR 1998 hat zu einem vermehrten Gebrauch außergerichtlicher Konfliktlösungsmethoden und zu einem Rückgang des Geschäftsanfalls bei den Gerichten geführt. ${ }^{375}$

296 Fraglich ist, ob in Deutschland die Regelungen der $\iint 91 \mathrm{ZPO}$ bereits de lege lata ähnliche Kostensanktionen zulassen können, ohne eine Gesetzesänderung herbeiführen zu müssen. Zwar gibt es auch im deutschen Recht bereits Durchbrechungen des „The winner takes it all“-Prinzips, ${ }^{376}$ doch lassen diese Regelungen aufgrund ihres Ausnahmecharakters, beschränkten Anwendungsbereichs und fehlender Vergleichbarkeit der Sachverhalte Analogieschlüsse nicht zu, ${ }^{377}$ so dass fraglich ist, ob darüber hinaus im Rahmen des $\ 91$ ZPO die Frage, ob eine Partei ein ernsthaftes und nicht von vornherein aussichtsloses Mediationsangebot der anderen Seite ungerechtfertigt zurückweist, für die Verteilung der Kostenlast bereits Berücksichtigung finden kann.

297 Einen möglichen Ansatzpunkt könnte die Auslegung des Begriffes der Notwendigkeit in $\ 91$ ZPO darstellen. ${ }^{378} \mathrm{Im}$ Rahmen des $\ 91$ ZPO werden Kosten für Handlungen nur dann als notwendig angesehen, wenn sie zur Zeit ihrer Vornahmen objektiv erforderlich und geeignet erscheinen, das im Streit stehende Recht zu verfolgen oder zu verteidigen, wobei für die Parteien bei Berücksichtigung ihrer vollständigen Interessen das Gebot wirtschaftlicher Prozessführung besteht. ${ }^{379}$ Dabei sind die Parteien aber nicht verpflichtet, besondere Anstrengungen zu treffen oder Risiken einzugehen, damit der unterliegende Gegner möglichst wenig Kosten erstatten muss. ${ }^{380}$

298 Die aus $₫ 278$ Abs. 2, 5 ZPO abzuleitende Förderung alternativer Streitbeilegungsmethoden und prozessverhütender Maßnahmen durch den Gesetzgeber wird im Wesentlichen damit begründet, dass zum einen ein außergerichtliches Verfahren im Erfolgsfall günstiger sein kann als der staatliche Rechtsschutz, zum anderen aber durch eine gütliche Einigung die Gesamtwohlfahrt gesteigert werden

\footnotetext{
${ }^{374}$ Halsey v. Milton Keynes General NHS Trust/Steel v. Halliday, [2004] EWCA (Civ) 576, Nr. 16 ff.

375 Greger, JZ 2002, 1020, 1021; Sobich, ZvglRWiss 103 (2004), 69, 84.

376 ss 93, 93 d, 94, 95, 96, 97 Abs. 2 ZPO.

377 Mankowski, ZKM 2004, 8, 9 f.

378 Hierzu ausführlich Mankonski, ZKM 2004, 8, $10 \mathrm{ff}$.

379 OLG Bremen NJW 1969, 142, 143; BayVGHAnwBL. 2000, 323, 324

380 Zimmermann, ZPO, \91 Rn. 15.
} 
kann. ${ }^{381}$ Dieses Ziel kann auch bei der Interpretation und Anwendung schon bestehender Vorschriften im Wege der Auslegung berücksichtigt werden. ${ }^{382}$ Es wäre daher zu überlegen, ob die Möglichkeit einer unter Umständen günstigeren konsensualen Streitbeilegungsmöglichkeit die Kosten für ein Klageverfahren als nicht notwendig erscheinen lassen. Allerdings darf die Berücksichtigung der gesetzgeberischen Förderung alternativer Streitbeilegungsmethoden im Wege der Auslegung nicht zu einem kompletten Bruch mit der bisherigen Auslegungspraxis führen; grundsätzliche Änderungen und fundamentale Wertentscheidungen wie die Kostenverteilungsregel des $\int 91 \mathrm{ZPO}$ müssen Aufgabe des Gesetzgebers bleiben. Die Förderung alternativer Streitbeilegungsmechanismen hat Auswirkungen auf die gesamte Justizstruktur und berührt geradezu die Fundamente der Rechtsdurchsetzung. 383

Bislang hatte der Kläger die Wahl zwischen verschiedenen Verfahren und erlitt keine Nachteile, wenn er statt eines Mahnverfahrens den Klageweg beschritt, ${ }^{384}$ so dass sich eine Partei durch die Wahl des Klageweges nicht dem Vorwurf fehlender Notwendigkeit der Kosten aussetzen brauchte, wenn sie nicht zu einer anderen Streitbeilegungsform gezwungen wurde. Durch die Neuregelung des $\int 278$ ZPO sollte ihr lediglich ein anderer Weg nahe gelegt werden. ${ }^{385}$ Dazu kommt, dass der Gesetzgeber im Rahmen des $\int 15 a$ EGZPO durchaus Möglichkeiten aufgezeigt hat, Parteien zu anderen Wegen zu zwingen, so dass es sich bei $\$ 278$ Abs. 5 ZPO um eine bloße Empfehlung handelt, die jedoch keine Kostensanktionen und Änderungen fundamentaler Wertungen der ZPO nach sich ziehen darf. Die Berücksichtigung der Frage, ob eine Partei ein ernsthaftes und nicht von vornherein aussichtsloses Mediationsangebot der anderen Seite ungerechtfertigt zurückweist, im Rahmen der Verteilung der Kostenlast würde daher die Grenze der zulässigen Weiterentwicklung des Rechts durch Auslegung überschreiten. Solange der Kläger noch nicht auf einen anderen Weg gezwungen ist, muss sich die Klage im Bereich des Notwendigen halten. ${ }^{386}$

\footnotetext{
381 Ausführlich Mankowski, ZKM 2004, 8, 10 f. m.w.N.

382 So z.B. im Rahmen des $₫ 114$ ZPO AG Bochum ZKM 2003, 233; im Ergebnis abgelehnt von OLG Hamm ZKM 2003, 232, ausführlich dazu unten Rn. 311. Mankowski, ZKM 2004, 8, 11 weist aber zugleich auf Strukturunterschiede zwischen \$ 91 und \$114 ZPO hin und hält daher anders als die Auslegung im Rahmen des $\int 91$ ZPO eine Berücksichtigung dieser Wertung im Rahmen des $₫ 114$ ZPO für zulässig.

383 Mankowski, ZKM 2004, 8, 11.

384 Bork, in Stein/Jonas, ZPO, $\$ 91$ Rn. 58; Steiner, in Wieczorek/Schütze, ZPO, $₫ 91$ Rn. 71.

385 Mankowski, ZKM 2004, 8, 11.

386 Mankowski, ZKM 1004, 8, 11 f.
} 
300 Auch wenn man an Parallelen etwa zum Verhältnis zwischen Abmahnung und gerichtlichem Verfahren im Bereich des unlauteren Wettbewerbs denkt, ergeben sich nichts anderes: Hier ist es für den Kläger zur Vermeidung von Kostennachteilen in der Regel erforderlich, dass er vor Anstrengung eines Prozesses den Schuldner zunächst abmahnt. Unterlässt er dies, so wird in der Praxis an das Versäumnis der Abmahnung regelmäßig eine Kostenbelastung des Gläubigers gemäß $\int 93$ ZPO geknüpft, wenn der nicht abgemahnte Schuldner im Prozess die Ansprüche des Gläubigers sofort anerkennt. ${ }^{387}$ Lediglich in einigen Ausnahmefällen vorauszusehende Erfolglosigkeit der Abmahnung ${ }^{388}$ sowie die Entbehrlichkeit der Abmahnung wegen Unzumutbarkeit ${ }^{389}$ - ist eine Abmahnung entbehrlich. Diese Fälle betreffen aber eindeutig die Kostenentscheidung im Rahmen von \93 ZPO und das vorprozessuale Verhalten, nicht das Verhalten während des Prozesses und nicht Entscheidungen im Rahmen von $\int 91$ ZPO. Die Vorschriften der $\iint 93$, 93d, 94, 95, 96, 97 Abs. 2 ZPO betreffen jeweils eine spezielle prozessuale Konstellation und zielen weder auf die Förderung einer allgemeinen Kooperationsbereitschaft inter partes, noch auf die Stärkung des Schlichtungsgedankens ab. 390 Dies verbietet sowohl Einzel- als auch Gesamtanalogien zu diesen Vorschriften.

301 Denkbar wäre allenfalls eine mediationsfreundliche Interpretation, soweit das Gesetz selbst in \91a ZPO das Gericht zu einer Billigkeitsentscheidung ermächtigt. Zwar darf hier nicht außer Betracht bleiben, dass auch im Rahmen des $\int 91 \mathrm{a}$ ZPO in der Regel die Maßstäbe der $\iint 91 \mathrm{f}$. ZPO herangezogen werden, ${ }^{391}$ doch eröffnet $\int 91 \mathrm{a} Z \mathrm{ZPO}$ dem Gericht einen allein durch sachfremde Erwägungen und Willkür begrenzten weitestmöglichen Spielraum, ${ }^{392}$ die auch die Heranziehung der in \93 ZPO niedergelegten Grundsätze ermöglicht ${ }^{393}$. Bereits in der älteren Rechtsprechung wurde zum Teil vertreten, dass das frühzeitige Abbrechen außer-

387 Teplitzky, Wettbewerbsrechtliche Ansprüche und Verfahren, 8. Auflage 2002, Kap. 41 Rn. 7; Stein/Jonas/Bork, ZPO J 93 III Rn. 16a; Baumbach/Hartmann, ZPO, \ 93 ZPO Rn. 67 ff.; HkZPO/Gierl, S 93 ZPO Rn. 17.

388 Siehe hierzu im Einzelnen Teplitzky, Wettbewerbsrechtliche Ansprüche und Verfahren, 8. Auflage 2002, Kap. 41 Rn. 23 ff.

389 Siehe hierzu im Einzelnen Teplitzky, Wettbewerbsrechtliche Ansprüche und Verfahren, 8. Auflage 2002, Kap. 41 Rn. 28 ff.

390 Althammer, JZ 2006, 69, 73; Greger/Engelhardt, ZKM 2004, 10; Baumann, IDR 2005, 9, 16.

391 Stein/Jonas/Bork, ZPO-Kommentar, \91a Rn. 32 f. Zöller/Vollkommer, ZPO-Kommentar, \91a Rn. 24; OLG Zweibrücken, NJW 1986, 939; OLG Hamm, NJW-RR 2005, 331 (obiter dictum); OLG Saarbrücken, OLGR 2004, 167, 168; OLG Jena, OLGR 2000, 367.

392 OLG Hamm, NJW-RR 2000, 212; Baumbach/Lauterbach/Albers/Hartmann/Hartmann, ZPO-

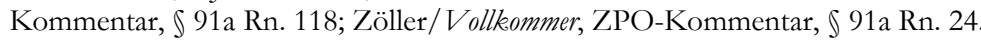

393 Zöller/Vollkommer, ZPO-Kommentar, J 91a Rn. 24; OLG Düsseldorf, NJW-RR 1996 905; OLG Hamm. NJW-RR 1986, 1121; OLG München, NJW-RR 1992, 731. 
gerichtlicher Vergleichsverhandlungen eine Kostenbelastung des Klägers nach sich zu ziehen vermag, selbst wenn seine Klage im Ergebnis Aussicht auf Erfolg ausweist. ${ }^{394}$ Die Regelung, dass die unterlegene Partei die gesamte Kostenlast zu tragen hat, kann daher für den Fall fehlender ernsthafter Bemühungen um außergerichtliche Streitbeilegung gleichsam flexibler interpretiert werden und kann somit für einen geringen Teilbereich auch für Mediationsverfahren fruchtbar gemacht werden. Eine mediationsfreundliche Auslegung muss jedoch an ihre Grenzen stoßen, die einen fundamentalen Systemwechsel in der Kostenpraxis der Gerichte zur Folge haben könnte.

Echte Anreize durch Kostensanktionen sind daher im gegenwärtigen Prozessrecht hingegen noch nicht möglich, ${ }^{395}$ sodass ist die Einführung einer entsprechenden Regelung, um deutliche Anreize zur Inanspruchnahme des gerichtlichen Mediationsangebotes zu setzen, zu empfehlen ist. Eine solche Kostensanktion würde die Überlegungen der Parteien zur Kostenverteilung und Erfolgsaussichten positiv im Sinne der gerichtsnahen Mediation beeinflussen. Eine Entscheidung für oder gegen die gerichtsnahe Mediation würde weniger an den Aussichten der Parteien im Prozess fest gemacht, sondern vielmehr den Schwerpunkt der Überlegungen von rechtlichen Bewertungen auf den Konflikt als solchen verschieben. Die Parteien hätten dann einen Anreiz, das Potential des Falles für die Mediation stärker auszuschöpfen. Finanzielle Sanktionen könnten dann eine wirksame und effektive Prävention unkooperativen Parteiverhaltens darstellen, gegen die auch aus rechtsstaatlicher Sicht keine Bedenken bestehen. ${ }^{396}$

Schwierigkeiten könnten sich dann bei einer gerichtlichen Überprüfung der Kostenverteilungsentscheidung ergeben, soll der Inhalt der Mediationsverhandlungen vertraulich behandelt werden. Die Erarbeitung eines Katalogs zur Bestimmung von Kriterien einer berechtigten Verweigerung oder eines berechtigten Abbruchs der Mediation würde hier auch den Parteien eine gewisse Voraussehbarkeit und Sicherheit über ihr eigenes Verhalten geben.

Allerdings können unter Umständen auch mildere Mittel zur Anreizsetzung, wie beispielsweise die Verpflichtung der Parteien zur Teilnahme an einer Informationssitzung zur gerichtsnahen Mediation, ${ }^{397}$ ausreichen.

\footnotetext{
394 OLG Nürnberg VersR 1969, 359; LG Berlin VersR 1968, 155, 906.

395 So auch schon Greger/Engelhardt, ZKM 2003, 4, 5; ähnlich auch Althammer, JZ 2006, 69, 74.

396 Wagner, ZKM 100, 103, der neben unkooperativem sogar von die Mediation sabotierendem

Verhalten spricht; Baumann, IDR 2005, 9, 15 im Hinblick auf Art. 6 EMRK und den Justizgewährleistungsanspruch.

397 Siehe dazu auch Duve, AnwBl 2004, 1, 4 f.; Ewig, ZKM 2002, 45, 46.
} 


\section{(6) Kostenanreize in anderen Verfahren (SGG)}

305 Probleme wirft ein Kostenanreiz für die Parteien vor allem im Bereich der Sozialgerichtsbarkeit auf: Hier entstehen Naturalpersonen gemäß \183 SGG keinerlei Gerichtskosten - gleichgültig, ob sie in dem jeweiligen Prozess obsiegen oder verlieren. Die Gerichtskosten fallen zur Schaffung von etwaigen Kostenanreizen somit von vornherein bei der Beteiligung von Naturalpersonen aus. Nichtsdestotrotz entstehen den Beteiligten die üblichen in einem Prozess anfallenden Kosten, wie zum Beispiel die Anwaltskosten. Gemäß \193 Abs. 1 SGG hat das Gericht darüber zu entscheiden, ob und in welchem Umfang die Beteiligten einander Kosten zu erstatten haben. In der Regel wird es hierbei als billig angesehen, dass derjenige die Kosten trägt, der unterliegt. ${ }^{398} \mathrm{Da}$ dies aber nur eine Billigkeitsregel ist, wäre es zwar durchaus möglich, die Kosten auch anders zu verteilen, sodass zumindest eine theoretische Möglichkeit eines Kostenanreizes auch für diese Konstellationen in der Sozialgerichtsbarkeit gegeben wäre. Ob dies in der Praxis für die Fälle der Prozesse, in denen es um Sozialleistungen geht, wünschenswert wäre, erscheint jedoch mehr als fraglich, da es in diesen Prozessen zum Teil um für die Beteiligten gravierende und in Grundrechtspositionen eingreifende Belange geht, so dass es unbillig erscheint, hier über Kostenanreize die Beteiligten zu einer Mediation zu bewegen.

306 Noch anders gestaltet sich die Situation, wenn erst gar keine Naturalpersonen an dem Verfahren beteiligt sind, sondern es sich um öffentliche Personen handelt. Hier entstehen die Gerichtskosten gemäß \197a SGG, der auf die Vorschriften der $\mathrm{VwGO}$ verweist. Eine Ermessenentscheidung über die Kosten scheidet hier von vornherein aus, sodass keinerlei Kostenanreize für die Mediation gesetzt werden können.

\section{dd) Kostenerstattung durch die Rechtsschutzversicherung}

307 Nicht nur im Falle eines streitigen Verfahrens, sondern auch in der gerichtsnahen Mediation im Zusammenhang mit einem gerichtlichen Verfahren sind die anfallenden Kosten durch eine bestehende Rechtsschutzversicherung zu tragen. Dies gilt nach dem Inkrafttreten des RVG auch für in der Mediation anfallende Rechtsanwaltskosten. Ob hingegen die in Zukunft anfallenden gerichtlichen Mediationskosten von der Rechtsschutzversicherung getragen werden, ist noch nicht abschließend geklärt. 399

\footnotetext{
398 Meyer-Ladewig/Keller/Leitherer/Mayer-Ladewig/Leitherer, SGG-Kommentar, \$193 SGG Rn. 12.

399 Siehe hierzu Rn. 160.
} 
Ergeben sich im Vergleich mit dem streitigen Verfahren vor Gericht zunächst keine negativen Anreize, das gerichtsnahe Mediationsangebot zu wählen, so besteht durch die aktuellen Rahmenbedingungen und das tatsächliche Verhalten der Rechtsschutzversicherer derzeit ein starker Anreiz für die Parteien, das Angebot der gerichtsnahen Mediation statt außergerichtliche Streitschlichtungsangebote zu wählen:

Zwar sind nach den Empfehlungen des Gesamtverbandes der Deutschen Versicherungswirtschaft e.V. (GDV) die Kosten eines Mediationsverfahrens gemäß den derzeit empfohlenen Allgemeinen Bedingungen für die Rechtsschutzversicherung (ARB 94 bzw. 2000) in bestimmten Fällen vom Versicherungsschutz gedeckt, ${ }^{400}$ doch sind für den Bereich der außergerichtlichen Mediation die Rechtsschutzversicherungen bislang nur zum Teil bereit, die Kosten für die Inanspruchnahme eines Mediators zu übernehmen. ${ }^{401}$ Es bleibt abzuwarten, ob die Versicherungen die Kosten für eine gerichtlich angeregte und im Gericht durchgeführte Mediation in der Zukunft bereitwilliger übernehmen.

Die sicher gewährleistete und nicht bloß empfohlene Übernahme der im Zusammenhang mit der gerichtsnahen Mediation entstandenen Kosten durch die Rechtsschutzversicherung stellt derzeit einen großen Vorteil der gerichtsnahen Mediation gegenüber anderen außergerichtlichen Streitschlichtungsangeboten dar. ${ }^{402}$

\section{ee) Prozess- und Mediationskostenhilfe}

Die Prozesskostenhilfe erfasst nach \114 ZPO (gem. \166 VwGO und \73 a Abs. 1 S. 1 SGG gelten die Vorschriften der Zivilprozessordnung über die Prozesskostenhilfe im Verwaltungsgerichts- und Sozialgerichtsverfahren entsprechend) die eigenen Kosten der Prozessführung und der gerichtlichen Gebühren und Auslagen. Da nun auch die Anwaltskosten in der Mediation durch den Gebührentatbestand der Nr. 3104 VV RVG gedeckt sind, werden diese Kosten auch von der Prozesskostenhilfe übernommen. Hier ergeben sich daher keine Unterschiede zum streitigen Verfahren.

\footnotetext{
400 Siehe hierzu Rn. 160.

401 Eine Anfrage hat ergeben, dass ein Drittel der Rechtsschutzversicherungen nicht bereit sind, die Kosten eines Mediationsverfahrens zu übernehmen, siehe FINANZtest 4/2004, 14.

402 Für normative Aspekte siehe unten Rn. 375.
} 
312 Werden in Zukunft gerichtliche Mediationskosten eingeführt, so ist eine der Prozesskostenhilfe entsprechende Mediationskostenhilfe unbedingt zu empfehlen, ${ }^{403}$ um keine negativen Anreize für die gerichtsnahe Mediation für bedürftige Parteien zu erzeugen.

313 Darüber hinaus ist aber auch das Setzen von positiven Anreizen für die Inanspruchnahme von vorgerichtlicher Mediation möglich. Nach jüngeren Entscheidungen ${ }^{404}$ kann das Ausschlagen eines ernsthaft gemeinten und nicht von vornherein aussichtslosen Mediationsangebots der anderen Partei oder von dritter Seite zu Gunsten einer Klage zum Vorwurf einer mutwilligen Prozessführung führen. Die Gewährung von PKH könnte daher langfristig auch an das ernsthafte Bemühen um eine nicht streitige Entscheidung geknüpft werden, die Bereitschaft der gegnerischen Partei zur Mediation natürlich vorausgesetzt.

314 Hier gilt aber zu bedenken, dass in diesen Fällen ein umfassendes Informationsangebot bzw. Informationsgespräch über die Mediation eingeführt wird, um die Partei über dieses Angebot aufzuklären. Der bloße Hinweis auf möglicherweise auch außergerichtliche Mediationsangebote kann hier nicht genügen.

\section{ff) Zeitlich bedingte finanzielle Anreize}

\section{(1) Anreize für den Kläger}

315 Ein Vorteil der gerichtsnahen Mediation gegenüber dem normalen streitigen Verfahren kann ferner darin liegen, dass die beteiligten Parteien schneller ein Ergebnis erzielen und so negative wirtschaftliche bzw. finanzielle Folgen langer Verfahrensdauer vermeiden können. Dadurch kann unter Umständen selbst dann ein Anreiz zur gerichtsnahen Mediation bestehen, wenn die klagende Partei mit einer hohen Wahrscheinlichkeit mit einem Obsiegen im Prozess rechnet.

\footnotetext{
403 Siehe hierzu Rn. 164.

404 AG Bochum ZKM 2003, 233; im Ergebnis abgelehnt von OLG Hamm ZKM 2003, 232; hinsichtlich des hier interessierenden Aspekts der Mutwilligkeit bestehen jedoch übereinstimmende Wertungen; siehe hierzu auch Mankowski, ZKM 2003, 197 ff.
} 


\section{(a) Entwertung eines Prozesserfolges durch eine überlange Verfah- rensdauer}

\section{(aa) Berücksichtigung der Prozessdauer durch Diskontierung}

Zunächst kommt eine Entwertung des Prozesserfolges für die obsiegende Partei durch eine überlange Verfahrensdauer in Betracht. ${ }^{405}$ Eine lange Prozessdauer bewirkt, dass der Kläger seine Leistung später erhält und der Beklagte die Leistung erst um die Prozessdauer verzögert erbringen muss. D.h. um einen Vergleich zwischen dem finanziellen Ergebnis des in der Mediation ausgehandelten Prozessvergleiches mit einem streitigen Urteil ziehen zu können, muss grundsätzlich der Erwartungswert der Auszahlung bei einem Obsiegen im streitigen Verfahren abdiskontiert werden.

\section{(bb) Schädigung des Klägers durch Brachliegen seines Kapitals}

Daneben kann das unter Umständen jahrelange Brachliegen von Geldforderungen aufgrund einer langen Verfahrensdauer einen weiteren finanziellen Verlust für den Berechtigten darstellen. Der Kläger kann über das ihm zustehende Geld nicht verfügen. Der Ausgang des Prozesses ist unsicher und auch der Zeitpunkt, wann der Kläger über das ihm zustehende Kapital verfügen kann, ist nicht berechenbar. Der Kläger ist somit in seinen Investitionsplänen eingeschränkt. Auch ist in den Fällen, in denen dem Kläger ein Anspruch auf Ersatz des entgangenen Gewinns gegen den Beklagten zustehen kann, dieser vor allem für Privatleute schwer zu beweisen. Ob und wie viel Gewinn der Kläger mit dem Geld hätte erzielen können, wenn er sofort über das Geld hätte verfügen können, ist nur schwer festzustellen. 406 Auch Ansprüche gegen den Staat wegen überlanger Verfahrensdauer aus $\int 839$ BGB erscheinen wenig erfolgversprechend. 407

\section{(cc) Durch überlange Verfahrensdauer verursachter Konkurs}

Ferner kommt in Betracht, dass eine überlange Verfahrensdauer unter Umständen eine Mitursache für den Konkurs des Klägers darstellen kann, wenn z.B. der Kläger einen berechtigten Anspruch durchsetzen will, um so einen drohenden Kon- 
kurs abzuwenden. ${ }^{408}$ Ebenfalls problematisch erscheint ein drohender Konkurs des Beklagten. Verfällt der Beklagte während des Prozesses in Konkurs, so ist der Kläger auf die Befriedigung seiner Forderung aus der Konkursmasse angewiesen. Er erhält dann nur einen bestimmten Prozentsatz der ursprünglichen Forderung. ${ }^{409}$ Dieses Risiko entfällt zwar nicht im Mediationsverfahren, ist aber wegen der in der Regel insgesamt kürzeren Verfahrensdauer bis zur Erzielung eines Ergebnisses eher abschätzbar.

\section{(dd) Überbrückung von langen Wartezeiten}

319 Eine Entwertung der Rechtsposition durch eine lange Verfahrensdauer kann sich insbesondere dann ergeben, wenn ein Anspruch auf Rente oder Unterhalt geltend gemacht wird. Der Kläger hat dann trotz berechtigten Anspruchs die Wartezeit bis zu einem Urteil zu überbrücken und ist gegebenenfalls auf die Unterstützung aus öffentlichen Mitteln, eventuell Leistungen aus freiwilligen Versicherungen angewiesen. ${ }^{410}$

\section{(b) Gefährdung der materiellen Richtigkeit des Urteils durch lange Verfahrensdauer}

320 Ferner ist zu berücksichtigen, dass eine lange Verfahrensdauer nicht nur das Urteil für den Kläger entwerten, sondern unter Umständen auch seine materielle Richtigkeit gefährden kann. Gegebenfalls kann dies dazu führen, dass die Rechtsdurchsetzung des Berechtigten ganz vereitelt wird, was wiederum dazu führt, dass er nicht nur eine berechtigte Forderung nicht durchsetzen kann, sondern auch noch gemäß \91 ZPO als unterliegende Partei die Prozesskosten der gegnerischen Partei tragen muss. Dies gilt für die die Beweislast tragende Partei insbesondere dann, wenn mit zunehmender Prozessdauer eine zutreffende Klärung des Sachverhalts und eine Aufklärung der Streitpunkte immer schwerer möglich wird, weil die Erinnerung der Parteien und Zeugen mit der Zeit verblasst, oder Zeugen in der Zwischenzeit versterben oder nicht mehr auffindbar sind. ${ }^{411}$

\footnotetext{
408 Gerking, in: Schmidtchen/Weth, Der Effizienz auf der Spur, 1999, S. 38, 39 f.

${ }^{409}$ Gerking, in: Schmidtchen/Weth, Der Effizienz auf der Spur, 1999, S. 38, 40.

410 Gerking, in: Schmidtchen/Weth, Der Effizienz auf der Spur, 1999, S. 38, 41.

411 Posner, 2 Journal of Legal Studies 399, 420 (1973); Schwab/Gottwald, Verfassung und Zivilprozeß, S. 57.
} 


\section{(c) Konsequenzen}

Die vorstehenden Ausführungen zu den Risiken einer überlangen Verfahrensdauer machen deutlich, dass ein starker Anreiz für den (risikoaversen) Kläger bestehen kann, das Verfahren zugunsten der gerichtsnahen Mediation auszusetzen und so schneller ein Ergebnis erzielen zu können, selbst wenn er dann nicht seine Maximalforderung durchsetzen kann und unter Umständen selbst dann, wenn er eigentlich einem Obsiegen im Prozess und dem Durchsetzen seines Anspruchs eine hohe Wahrscheinlichkeit beimisst.

Zudem ist zu berücksichtigen, dass die ebengenannten finanziellen Nachteile einer langen Prozessdauer in der Regel die finanzschwächere Partei treffen und dadurch die Unterschiedlichkeit der Prozesschancen verstärken. ${ }^{412}$

\section{(2) Anreize für den Beklagten}

Aus den oben dargestellten Nachteilen folgen zugleich Vorteile für den Beklagten durch eine lange Verfahrensdauer. Der Beklagte kann einen Anreiz haben, die lange Verfahrensdauer zu seinen Gunsten auszunutzen und somit zunächst kein Interesse daran, schneller ein Ergebnis durch die Durchführung einer Mediation herbeizuführen.

\section{(a) Kreditgewährung als negativer Anreiz für die Mediation}

Für den Beklagten besteht die Möglichkeit, die Erfüllung des Anspruchs durch einen Prozess hinauszuzögern. Zwar entstehen dem Schuldner dadurch Verzugszinsen und ferner hat er die Gerichtskosten zu tragen, doch kann der Beklagte dann unter Umständen vorteilhaft prozessieren, wenn ein solcher vom Kläger erzwungener Kredit günstiger ist als ein normaler Bankkredit. ${ }^{413}$

Allerdings sind bei diesen Überlegungen zugleich eventuelle Sanktionen des „Zwangskreditgebers" zu berücksichtigen. Insbesondere in langfristigen Geschäftsbeziehungen ist das Verhältnis dann gänzlich gestört. Es entstehen Transaktionskosten (insbesondere Suchkosten), um neue Geschäftsbeziehungen aufzubauen. Außerdem kann ein solches Verhalten des Beklagten seine Reputation im Geschäftsleben schädigen, so dass letztlich auch ein Interesse des Beklagten am

412 Schwab/Gottwald, Verfassung und Zivilprozeß, S. 57; Bernstein, 141 University of Pennsylvania Law Review 2169, 2193 (1993), Fn. 101.

413 Für eine vertiefte Darstellung siehe Adams, Ökonomische Analyse des Zivilprozesses, S. 60 ff.; Wein, Recht durch Rechtsanwälte?, S. 237. 
Erhalt der Beziehung bestehen kann und dieser sich somit auch auf eine Mediation zwecks Erzielung eines schnelleren Ergebnisses einlassen würde. ${ }^{414}$

\section{(b) Zeitgewinn durch den Prozess als negativer Anreiz für die Mediation}

326 Unabhängig von der finanziellen Wirkung des Hinauszögerns eines Prozesses kann auch der bloße Zeitgewinn für den Beklagten einen Vorteil und somit einen negativen Anreiz für die Zustimmung zur gerichtsnahen Mediation darstellen. So kann möglicherweise ein Mieter erst mit großer Zeitverzögerung zur Aufgabe der Wohnung gezwungen werden, selbst wenn das Kündigungsverlangen ohne Zweifel berechtigt ist. Der Mieter beharrt dann insbesondere auf die bisherige Wohnung, wenn er für eine andere Wohnung einen höheren Mietzins zahlen müsste oder gar keine neue Wohnung findet. Der beklagte Mieter realisiert in diesem Fall einen Verzugsgewinn. ${ }^{415}$

\section{(c) Zermürben des Gegners durch die lange Prozessdauer als negativer Anreiz für die Mediation}

327 Der Beklagte kann ferner einen Anreiz haben, den Prozess in die Länge zu ziehen und dadurch den Prozessgegner, also den Kläger, zu einem für ihn ungünstigen Vergleich zu drängen. ${ }^{416}$ Diese Gefahr besteht insbesondere dann, wenn der Beklagte die finanzstärkere Partei ist und der Kläger auf die Durchsetzung des Anspruchs besonders angewiesen ist. ${ }^{417}$

\section{(d) Gefährdung der materiellen Richtigkeit des Urteils durch lange Verfahrensdauer}

328 Hält der Beklagte ein Obsiegen im Prozess für wahrscheinlich, so besteht für ihn durch eine lange Verfahrensdauer - wie auch schon oben für den Kläger erwähnt - ebenfalls die Gefahr einer Gefährdung der materiellen Richtigkeit der Urteilsfindung und somit ein Anreiz, durch die Mediation ein schnelleres Ergebnis zu erzielen.

\footnotetext{
414 Siehe auch Bernstein, 141 University of Pennsylvania Law Review 2169, 2193 f., Fn. 101 (1993).

415 Wein, Recht durch Rechtsanwälte?, S. 237.

416 Gerking, in: Schmidtchen/Weth, Der Effizienz auf der Spur, S. 38, 45.

417 Schwab/Gottwald, Verfassung und Zivilprozeß, S. 57.
} 


\section{gg) Ersparnis sonstiger Kosten}

\section{(1) Transaktionskosten}

Ein Anreiz zur Durchführung der gerichtsnahen Mediation kann für beide Parteien in einer Ersparnis von Transaktionskosten liegen. Durch die Durchführung einer konsensualen Streitbeilegung können neben den Kosten, die mit der Vorbereitung und Führung eines Rechtsstreits verbunden sind, vor allem auch die mit einem Prozess zusammenhängenden immateriellen Kosten durch Ärger, Verlust an Lebensfreude etc. reduziert werden.

\section{(2) Reputationsbezogene Kosten}

Ferner kann durch die vertrauliche Behandlung des Konfliktes im Rahmen der Mediation eine Einbuße an Reputation vermieden werden, die ebenfalls mit Kosten verbunden wäre, sei es durch Einnahmenausfall, sei es durch zusätzlich erforderliche Aufwendungen, um die Reputation wieder herzustellen.

Im Vergleich zu sonstigen außergerichtlichen Mediationsverfahren entfallen bei gerichtsnaher Mediation auch die Kosten, die mit einer Entscheidung für die außergerichtliche Mediation und die Überzeugung der gegnerischen Partei verbunden wären. Für die Partei, die die außergerichtliche Mediation vorschlägt besteht oft die Gefahr, dass die andere Partei diesen Vorschlag als Signal einer schwachen Rechtsposition bzw. allgemein als Schwäche der Partei ansehen könnte. ${ }^{418}$ Durch den Vorschlag des Richtermediators bestehen diese Gefahr und der Verlust an Reputation durch den Gegner nicht. Daher kann auch keine Abwertung der Rechtsposition erfolgen.

\section{(3) Erhalt der Parteibeziehungen}

Insbesondere der Erhalt langfristiger Geschäftsbeziehungen oder auch persönlicher Beziehungen kann für die Beteiligten Parteien einen Anreiz darstellen, sich auf die konsensuale Streitbehandlung einzulassen und die Beziehungen dadurch zu erhalten. Die Sicherung langfristiger Geschäftsbeziehungen (z.B. Zulieferungsverhältnis) erspart die Aufwendung weiterer Transaktionskosten (insbesondere Suchkosten für neue Geschäftsbeziehungen, gegebenenfalls Kosten für die Umstellung der Produktion/Anpassung an den neuen Geschäftspartner).

418 Bernstein, 141 University of Pennsylvania Law Review 2169, 2192 (1993). 


\section{(4) Vermeidung von Folgeprozessen}

333 Auch kann ein Anreiz für Mediation in der Vermeidung von Folgeprozessen, die ebenfalls mit Kosten verbunden sind, bestehen. Dies gilt insbesondere im Fall von langfristigen Beziehungen. Für den bilanzierenden Kaufmann schlägt sich dies konkret darin nieder, dass möglicherweise langjährige Rückstellungen für Prozesskosten vermeiden kann. ${ }^{419}$

\section{hh) Abwägung der finanziellen Anreize und rechtspolitische Hand- lungsempfehlungen}

334 Die wie eben dargestellt offensichtliche ungleiche Verteilung der Vorteile der langen Verfahrensdauer bzw. einer schnelleren Erzielung eines Ergebnisses durch die Mediationsverhandlungen stellt eine Barriere für den Eintritt in Mediationsverhandlungen dar ${ }^{420}$ und hat daher Einfluss auf den von beiden Parteien gebildeten Erwartungswert eines streitigen Verfahrens bzw. der gerichtsnahen Mediation.

335 Wie oben dargelegt bestehen Anreize für eine Beschleunigung der Erzielung eines Ergebnisses durch die gerichtsnahe Mediation insbesondere für den Kläger, der einem Obsiegen im Prozess und dem Durchsetzen seines Anspruchs eine hohe Wahrscheinlichkeit beimisst und gleichzeitig bezüglich der oben dargestellten Gefahren bei langer Verfahrensdauer risikoavers ist.

336 Ob die Gefahr einer langen Prozessdauer und ihrer negativen Folgen den oben angesprochenen Anreiz durch die Verteilung der Kostenlast (Anreiz für den Kläger, der einem Obsiegen im Prozess eine hohe Wahrscheinlichkeit beimisst, um die Kostenlast gemäß \91 auf den unterliegenden Gegner abwälzen zu können und zudem auch keine zusätzlichen Kosten für die anwaltliche Begleitung in der Mediation selbst tragen zu müssen) ausgleicht, hängt von der individuellen Präferenzordnung, den unterschiedlichen subjektiven Wahrscheinlichkeiten hinsichtlich eines Prozesserfolges und der Risikoaversität der Partei ab. Ein pauschales Urteil ist nicht möglich. Zudem bestehen darüber hinaus evtl. noch weitere Anreize durch eine Ersparnis von Transaktionskosten und weitere immaterielle Aspekte, auf die später noch eingegangen wird. Diese Anreize sind alle im Einzelfall gegeneinander abzuwägen.

337 Gleiches gilt auch für den Beklagten. Schätzt er die Wahrscheinlichkeit, im Prozess zu unterliegen und die Kostenlast gemäß $\ 91 \mathrm{ZPO}$ zu tragen, als hoch ein, so

\footnotetext{
${ }^{419}$ Ditges, IDR 2005, 74, 77.

420 Bernstein, 141 University of Pennsylvania Law Review 2169, 2193 f., Fn. 101 (1993).
} 
besteht mit Blick auf die Gerichts- und Anwaltskosten für den Beklagten eher ein Anreiz zur gerichtsnahen Mediation, der allerdings von einem gegenläufigen Anreiz durch die Vorteile einer langen Verfahrensdauer ausgeglichen werden kann. Auch diese Abwägung hängt dann von den persönlichen Präferenzen, der subjektiven Wahrscheinlichkeit und Risikoaversität, dem Erwartungswert der Transaktionskosten und weiteren immateriellen Anreizen ab und muss daher im Einzelfall getroffen werden.

Zwar können diese zeitlich bedingten finanziellen Anreize durch gesetzliche Regeln nicht direkt beeinflusst werden, doch ist eine indirekte Beeinflussung durch Kostensanktionen durch einen geänderten \91 ZPO möglich. Hier kann auf die Ausführungen zu einer Änderung des $\int 91$ ZPO verwiesen werden. Ein solcher Kostenanreiz würde die Gefahr des eben beschriebenen taktischen Verhaltens einer Partei, der an einer Verzögerung des Prozesses gelegen ist, stark verringern und eine schnellere Einigung durch ein Mediationsverfahren unterstützen.

\section{b) Endgültigkeit des Mediationsverfahrens}

Im Hinblick auf die Effektivität der Mediation als Verfahren zur Beilegung rechtlicher Konflikte ist zunächst darauf hinzuweisen, dass das Mediationsverfahren im Vergleich zu einem gerichtlichen Verfahren keine Endgültigkeit hat. Durch die Mediation wird ein Streit nur beigelegt, wenn es den Konfliktparteien gelingt, sich mit Hilfe des Mediators über die streitigen Punkte zu einigen. Da es sich bei der Mediation um ein uneingeschränkt freiwilliges Verfahren handelt, steht es den Parteien jederzeit frei, die Verhandlungen abzubrechen, so dass das Ergebnis einer Mediation ebenso gut die Aufgabe des Mediationsvefahrens sein kann. Im Vergleich zum gerichtlichen Verfahren, bei dem die Parteien die Garantie einer - wie auch immer gestalteten - Entscheidung ihres Konflikts haben, kann sich die Mediation daher insoweit als ineffektiv erweisen, als im Falle eines Scheiterns der Verhandlungen Zeit und Kosten umsonst aufgewandt wurden. Eventuell können aber selbst gescheiterte Mediationsverhandlungen insoweit von Nutzen sein, als sie sich auf den Prozessverlauf und die langfristige Gestaltung der Beziehungen der Parteien zu einander positiv auswirken können.

\section{c) Durchsetzbarkeit des Mediationsergebnisses}

Die Frage der Effektivität des Mediationsverfahrens im Vergleich zum gerichtlichen Verfahren stellt sich darüber hinaus auch im Hinblick auf die Durchsetzbarkeit einer in der Mediation getroffenen Vereinbarung. Mediationsverfahren und 
die darin getroffenen Abschlussvereinbarungen liefern in der Regel pareto ${ }^{421}$ überlegene Konfliktlösungen im Sinne einer win-win-Situation ${ }^{422}$ auf zunächst einmal freiwilliger Basis. Bezüglich einer in der Mediation erarbeiteten Konfliktlösung wird daher angenommen, dass die Parteien sehr viel eher als im Fall einer für sie durch einen Dritten getroffenen Entscheidung bereit sind, sich an die getroffenen, von ihnen selbst entwickelten Vereinbarungen zu halten. ${ }^{423}$ Mit Blick auf die der ökonomischen Analyse des Rechts zugrunde gelegte Annahme des rationalen und eigennützigen Verhaltens ist jedoch zu beachten, dass selbst eine solche pareto-überlegene Lösung nicht gegen einen Bruch der Vereinbarung immun ist. ${ }^{424}$ Um negative Anreize zu vermeiden, ist eine Absicherung der Durchsetzbarkeit der in der Mediation getroffenen Vereinbarung daher unbedingt erforderlich.

341 Bei den in der Mediation erzielten Ergebnissen handelt es sich häufig um einen materiell-rechtlichen Vergleich gemäß \ 779 S. 1 BGB. Damit dieser rechtlich bindende Vertrag Bestand hat und von den Parteien beachtet wird, ist es erforderlich, dass er gerichtlich durchsetzbar ist. Da jedoch auch ein solcher Prozess ein gewisses Prozessrisiko birgt und mit Zeitaufwand verbunden ist, ist es im Interesse der Parteien, dass noch weitere Maßnahmen zur Sicherung der Vertragserfüllung ergriffen werden. Zu denken wäre in diesem Zusammenhang an die Einbeziehung materiell-rechtlicher Sanktionen in den in der Mediation geschlossenen Vergleich. ${ }^{425}$

342 Für die gerichtlichen Mediationsangebote in den öffentlich-rechtlichen Gerichtsbarkeiten sind jedoch die Möglichkeiten, eine rechtlich verbindliche Vereinbarung zu schließen, gegenüber den Möglichkeiten für einen Vergleichsschluss im Zivilrecht deutlich eingeschränkt. ${ }^{426}$ Gleichwohl können die Parteien aber auch in der gerichtsnahen Mediation einen Vollstreckungstitel erlangen. Hierbei stehen den Parteien zur Absicherung der Durchsetzbarkeit der in der gerichtsnahen Mediati-

${ }^{421}$ Eine Regelung wird dann pareto-effizient genannt, wenn sie aus der Sicht der Parteien nicht mehr dergestalt verbessert werden kann, dass zumindest eine Partei noch einen Vorteil erzielt, ohne dass die andere Partei hierdurch einen Nachteil erleidet; vgl. Schumann/Meyer/Ströbele, Grundzüge der mikroökonomischen Theorie, S. 35 f.

422 Im Gegensatz zu einem Nullsummenspiel, bei dem bei jedem Ereignis ein Spieler eine Auszahlung erhält, die der andere Spieler bezahlen muss, bezeichnet eine win-win-Situation in der Spieltheorie einen Zustand, in der beide Spieler, d.h. also die Parteien, positive Auszahlungen erhalten.

423 Bübring-Uble, Arbitration and Mediation in International Business, S. 342; Menkel-Meadow, 19 Florida State University Law Review, 1, 12 (1991); Pearson, 7 The Justice System Journal, 420, 433 (1982).

${ }^{424}$ Weigel, in: FS Ott, S. 491, 510.

425 Siehe hierzu Rn. 78.

426 Siehe hierzu Rn. 80. 
on getroffenen Vereinbarung die gleichen Instrumente wie im streitigen Prozess zur Verfügung, ${ }^{427}$ so dass sich für die Parteien letztlich unter diesem Aspekt keine negativen Anreize für die Wahl der gerichtsnahen Mediation ergeben.

\section{d) Vertraulichkeit des Mediationsverfahrens}

Der Erfolg einer Mediation wird im Wesentlichen davon bestimmt, dass die Parteien offen miteinander kommunizieren, und zwar nicht nur im Hinblick auf ihre Vergleichsbereitschaft, sondern auch bezüglich der hinter ihren jeweiligen Positionen verborgenen Interessen und Bedürfnisse. ${ }^{428} \mathrm{Da}$ jedoch nicht jedes Mediationsverfahren mit einer gütlichen Einigung enden muss, besteht für die Parteien die Gefahr eines Missbrauchs der in der Mediation mitgeteilten vertraulichen Informationen, insbesondere im geschäftlichen Verkehr, aber auch im Bereich ihrer privaten Beziehungen. ${ }^{429}$ Hier muss ein strategisches Verhalten befürchtet werden, dass eine Partei ohne echte Einigungsabsicht die systemimmanente Offenheit in der Mediation als taktisches Mittel zum Ausspionieren der anderen Partei nutzt und sich die Parteien dadurch Nachteilen im Hinblick auf den Fortgang des gerichtlichen Verfahrens aussetzen. 430

Wesentliche Voraussetzung für eine erfolgreiche Mediation wie aber auch die Bereitschaft zur Mediation überhaupt, ist daher der Schutz der Vertraulichkeit der in der Mediation offenbarten Informationen.

Der Richtermediator ist bereits aufgrund seiner beruflichen Stellung zur Verschwiegenheit verpflichtet. Darüber hinaus ergeben sich für den Richter nur vereinzelt Verpflichtungen, Informationen beispielsweise Straftaten betreffend, die er in einem Prozess erlangt, an die Staatsanwaltschaft oder andere Stellen weiterzuleiten. So sieht lediglich $\int 183$ GVG vor, dass ein Richter verpflichtet ist, in der Sitzung begangene Straftaten zu Protokoll zu nehmen und dies der zuständigen Behörde mitzuteilen. Damit sind solche Taten, von denen das Gericht lediglich in der Sitzung Kenntnis erlangt, die jedoch außerhalb des zeitlichen und örtlichen Anwendungsbereichs der Sitzung begangen wurden, von der Feststellungs- und Anzeigepflicht nicht erfasst; diesbezüglich ist das Gericht lediglich berechtigt,

\footnotetext{
427 Siehe hierzu Rn. 89.

428 Gray, 36 Osgoode Hall Law Journal 667, 671 (1998).

${ }^{429}$ Gray, 36 Osgoode Hall Law Journal 667, 671 (1998).

${ }^{430}$ Für die außergerichtiche Mediation: Weigand, BB 1996, 2106, 2108; Casper/Risse, ZIP 2000, 437, 440; Köper, Die Rolle des Rechts im Mediationsverfahren, S. 30.
} 
Straftaten wie jedermann zur Anzeige zu bringen. ${ }^{431}$ Aus \2 EGGVG ergibt sich darüber hinaus, dass $\int 183$ GVG nur dann einschlägig ist, wenn die Straftat in einer Sitzung der ordentlichen streitigen Gerichtsbarkeit begangen wird. Mediation als einvernehmlich bindendes Konfliktlösungsmodell hingegen liegt gerade außerhalb des streitigen Verfahrens und impliziert so bereits begrifflich die Nichtanwendbarkeit des $\int 183$ GVG. Daneben ergeben sich lediglich aus der Anordnung über Mitteilungen in Zivilsachen (MiZi) für bestimmte Delikte, in concreto Schwarzarbeiter-, Ausländer- sowie Steuerstrafsachen, Anzeigepflichten. Der Geltungsbereich der MiZi umfasst jedoch - ähnlich dem GVG - nur Verfahren der streitigen Zivilgerichtsbarkeit und der freiwilligen Gerichtsbarkeit und somit gerade nicht die Mediation als streitschlichtendes Verfahren. Außerhalb dieser Normen besteht für den Richter lediglich die sich für jeden Bürger aus \ 138 StGB ergebende Anzeigepflicht geplanter Straftaten nach dem dort angegebenen Katalog. Daraus folgt, dass der Mediator zwar Straftaten, welche während der Mediation begangen oder bekannt werden, zur Anzeige bringen kann, eine Pflicht hierzu jedoch lediglich für die Katalogstraftaten des \ 138 StGB konstatiert werden kann.

346 Für die Parteien, für die eine solche berufliche Pflicht in der Regel nicht besteht, kann eine Verschwiegenheitspflicht jedoch vertraglich herbeigeführt werden. ${ }^{432}$ Scheitern die Mediationsverhandlungen, so kann ein umfassender Schutz der Vertraulichkeit der Mediationsverhandlungen im nachfolgenden Gerichtsverfahren mit Mitteln des Vertragsrechts nicht hergestellt werden. ${ }^{433}$ Eine gesetzliche Regelung des Vertraulichkeitsschutzes in der gerichtsnahen Mediation ist daher dringend zu empfehlen, sollen negative Anreize für die Parteien vermieden werden. ${ }^{434}$

347 Eine gesetzliche Sicherung der Vertraulichkeit im Mediationsverfahren hat Signalwirkung für die Parteien und fördert somit die Offenheit im Gespräch und damit auch den Erfolg des Mediationsverfahrens. Mögliche Sanktionen bei Verletzung der Vertraulichkeitspflicht durch die Parteien könnten zudem vor strategischem Verhalten bzw. Missbrauch der Mediation zur bloßen Erlangung vertraulicher Informationen abschrecken.

\footnotetext{
431 MünchKommZPO/Wolf, ZPO-Kommentar, \183 GVG Rn. 3; Kissel/Mayer, GVG-

Kommentar, J 183 Rn. 1 f.; BayObLG NJW 1968, 56.

432 Siehe hierzu Rn. 109.

433 Siehe hierzu Rn. 110.

434 Näher hierzu oben Rn. 119.
} 


\section{e) Gefahr von Rechtsverlusten}

Nachteile und damit negative Anreize können sich für die Parteien nicht nur aufgrund eines möglichen Missbrauchs vertraulicher Informationen ergeben, sondern auch daraus, dass mit der Durchführung eines Mediationsverfahrens unter Umständen der Verlust von Rechtspositionen verbunden sein kann. Nachfolgend sollen daher mögliche mit der Durchführung eines Mediationsverfahrens verbundene Risiken, namentlich durch Zeitablauf drohende Rechtsverluste, der Verlust von mit der Rechtshängigkeit der Sache verbundenen Privilegien sowie die Gefahr der Verschiebung von Rechts- und Vermögenspositionen, erörtert und im Hinblick auf ihre Relevanz auch im Bereich der gerichtsnahen Mediation untersucht werden.

\section{aa) Durch Zeitablauf drohende Rechtsverluste}

\section{(1) Verjährung}

Nachteile bezüglich einer Verjährung der geltend gemachten Ansprüche brauchen die Parteien durch die Wahl der gerichtsnahen Mediation aufgrund der Verjährungshemmung nicht zu befürchten. ${ }^{435}$

\section{(2) Ablauf von Ausschlussfristen}

Da das gerichtsnahe Mediationsverfahren zumeist erst nach Rechtshängigkeit angestrengt wird und folglich von etwaigen Ausschlussfristen bedrohte Ansprüche bereits geltend gemacht wurden, ist die Gefahr des Ablaufs von gerichtlichen Ausschlussfristen gering. ${ }^{436}$ Für die von der Klage umfassten Ansprüche besteht daher kein negativer Anreiz.

In den Fällen, in denen es im Mediationsverfahren um Ansprüche geht, die nicht zur ursprünglichen Klage gehörten, ist jedoch diese Gefahr gegeben. Hier kann derjenigen Partei, die sich auf einen solchen Ablauf einer Ausschlussfrist beruft, jedoch die Einrede der unzulässigen Rechtsausübung nach $\ 242$ BGB entgegengehalten werden. ${ }^{437}$ Zur Klarstellung und als positiver Anreiz ist gleichwohl auch hier die Einführung einer gesetzlichen Regelung zu empfehlen, die für Ausschluss-

\footnotetext{
435 Siehe hierzu Rn. 99.

436 Siehe hierzu Rn. 101.

437 Siehe hierzu ausführlich Rn. 101.
} 
fristen eine Fristhemmung für die Dauer von Mediationsverhandlungen vorsieht. ${ }^{438}$

\section{bb) Verlust von mit der Rechtshängigkeit der Sache verbundenen Rechtsvorteilen}

\section{(1) Materiell-rechtliche Wirkungen der Rechtshängigkeit}

352 Im Gegensatz zur nicht von $\int 278$ Abs. 5 Satz 2 ZPO veranlassten Mediation bleiben dem Gläubiger im gerichtsnahen Mediationsverfahren die durch die Rechtshängigkeit einer Sache herbeigeführten materiell-rechtlichen Ansprüche ${ }^{439}$ bestehen, da das Mediationsverfahren nach $\ 278$ Abs. 5 S. 2 und 3 ZPO erst während des gerichtlichen Verfahrens und damit nach Rechtshängigkeit eingeleitet wird. ${ }^{440}$ Die nicht zum Klagegegenstand gehörenden Ansprüche fallen hier jedoch heraus, da sie mangels Klageerhebung nicht rechtshängig geworden sind. ${ }^{441}$ Diese Vorteile gelten für die gerichtsinterne und die von $\$ 278$ Abs. 5 Satz 2 ZPO veranlasste außergerichtliche Mediation aber gleichermaßen gegenüber einer vorgerichtlichen Mediation.

\section{(2) Prozesstaktische Vorteile}

353 Aufgrund der psychologischen Komplexität vieler Konflikte erscheint die Einbeziehung vom Konflikt betroffener Dritter in die gerichtsnahe Mediation sinnvoll. Während für das streitige Verfahren hier die Möglichkeit besteht, im Wege einer Streitverkündung die Nebeninterventionswirkung herbeizuführen, scheidet die Einbeziehung Dritter für die gerichtsnahe Möglichkeit aus, da die Nebeninterventionswirkung gerade nur durch rechtskräftiges Sachurteil, nicht jedoch durch einen Prozessvergleich herbeigeführt werden kann. ${ }^{442}$ Zwar wäre eine der Streitverkündung ähnliche Regelung für die gerichtsnahe Mediation wünschenswert, doch eine solche Regelung muss als Zwangslösung zur Einbeziehung Dritter selbstverständlich für die Mediation, bei der die Freiwilligkeit und der Konsens der beteiligten Parteien wesensimmanent sind, ausscheiden. Dadurch kann die gerichtsnahe Mediation gegenüber dem streitigen Verfahren für die Parteien gegebenenfalls als nachteilig erscheinen.

\footnotetext{
438 Siehe hierzu ausführlich Rn. 104.

439 Siehe im Einzelnen Rn. 105.

440 Siehe hierzu Rn. 105.

441 Siehe hierzu Rn. 105.

442 Siehe hierzu Rn. 106.
} 


\section{cc) Gefahr der Verschiebung von Rechts- und Vermögenspositionen}

Rechtsverluste durch eine Verschiebung von Rechts- oder Vermögenspositionen sind für die gerichtsnahe Mediation in nicht höherem Maße zu erwarten als für das streitige Gerichtsverfahren, ${ }^{443}$ so dass hier kein negativer Anreiz besteht.

\section{f) Zufriedenheit mit Verfahren und Ergebnis der Mediation}

Schließlich ist die Zufriedenheit der Konfliktparteien wesentliche Voraussetzung dafür, dass gerichtsnahe Mediation als Angebot der Justiz auf Akzeptanz trifft. Es wird angenommen, dass Verfahrensbeteiligte mit gerichtsnah angebotenen Formen alternativer Streitbeilegung in der Regel zufriedener sind als mit herkömmlichen Gerichtsverfahren. ${ }^{444}$ Untersuchungen zu verschiedenen Formen alternativer Streitbeilegung haben zudem gezeigt, dass an Mediationsverfahren beteiligte Konfliktparteien typischerweise besonders zufrieden sind. ${ }^{445}$ Der Grad der Zufriedenheit ist in diesem Zusammenhang nicht als eigenständiger Qualitätsmaßstab anzusehen, sondern vielmehr als Hinweis darauf, ob und inwieweit sich das Potenzial der Mediation realisiert. ${ }^{446}$ So wird sich die Zufriedenheit regelmäßig auf einzelne Aspekte des Mediationsverfahrens wie beispielsweise die Ersparnis monetärer sowie auch emotionaler Kosten beziehen. ${ }^{447}$ Am häufigsten wird die Zufriedenheit der Parteien jedoch auf Verfahren und Ergebnis der Mediationsverhandlungen bezogen..$^{48}$

\section{aa) Mediationsverfahren}

Die Flexibilität des Mediationsverfahrens wird als einer der wesentlichen Vorteile der Mediation gegenüber herkömmlichen Gerichtsverfahren gesehen. ${ }^{449}$ Die Parteien haben in der Mediation die Möglichkeit, mit Hilfe des Mediators das Verhandlungsprocedere ihren Bedürfnissen entsprechend selbst zu gestalten ${ }^{450}$ und

\footnotetext{
443 Siehe hierzu Rn. 107.

${ }^{444}$ Boulle/Nesic, Mediation: Principles, Process, Practice, S. 382 f.; die Abhängigkeit vom Parteieindruck des fairen Verfahrens aufzeigend Lande, UCLA Law Review 69 (2000), 69, 118 f.

445 Tyler, 66 Denver University Law Review, 419, 429 (1989).

446 Breidenbach, Mediation: Struktur, Chancen und Risiken von Vermittlung im Konflikt, S. $190 \mathrm{ff}$.

447 Bush/Folger, The Promise of Mediation: Responding to Conflict Through Empowerment and Recognition, S. 16 f.; siehe auch Gamm/Patera, IDR 2005, 59, 63.

448 Tyler, 66 Denver University Law Review, 419, 429 (1989).

449 Duve, Mediation und Vergleich im Prozess, S. 89; Risse, NJW 2000, 1614, 1618.

450 Boulle/Nesic, Mediation: Principles, Process, Practice, S. 35; Pryles, Assessing Dispute Resolution Procedures (1996) 7 The American Review of International Arbitration, 267, 278.
} 
von dem starren, im Prozess durch Klageanträge und wechselseitigen Parteivortrag geförderten Positionsdenken abzurücken, ${ }^{451}$ indem sie ihre tatsächlichen Beweggründe, Interessen und Bedürfnisse in die Mediationsverhandlungen mit einzubeziehen. ${ }^{452}$ Das gegenseitige Verständnis der Parteien für einander und die damit verbundene Chance einer einvernehmlichen, ihren jeweiligen Interessen entsprechenden Lösung des Konflikts kann so verbessert werden. Darüber hinaus kann die Eigenverantwortlichkeit der Parteien im Hinblick auf das Verfahren und den Gegenstand der Mediationsverhandlungen wesentlich zur Zufriedenheit der Parteien mit dem Mediationsverfahren beitragen. ${ }^{453}$ Die Parteien erleben das Verfahren der Mediation als fairer und persönlicher als herkömmliche Gerichtsverfahren. 454

\section{bb) Mediationsergebnis}

357 Die Mediation lässt darüber hinaus auch im Hinblick auf das Ergebnis der Verhandlungen sehr viel flexiblere und interessengerechtere Lösungen zu. Im Gegensatz zu einem Richter im streitigen Gerichtsverfahren ist der Mediator nicht darauf beschränkt, den geltend gemachten Anspruch entweder zuzusprechen oder abzuweisen ${ }^{45}$ und braucht sich bei der Konfliktlösung auch nicht ausschließlich an einem in der Vergangenheit liegenden und unter bestimmte Rechtsnormen subsumierbaren Sachverhalt zu orientieren. ${ }^{456}$ Er kann die Parteien vielmehr dabei unterstützen, auch für die Zukunft ihre jeweiligen Rechte und Pflichten zu regeln, ${ }^{457}$ was mit Blick auf die Gestaltung ihrer zukünftigen Beziehungen für die Parteien oftmals von sehr viel größerem Interesse ist. ${ }^{458}$ In die in der Mediation getroffene Abschlussvereinbarung können dann insbesondere auch Angelegenheiten mit einbezogen werden, die nicht zum Streitgegenstand der ursprünglichen Klage gehören und auch keine rechtliche Relevanz aufweisen. Diese Möglichkeit, die Lösung des Konflikts den individuellen Bedürfnissen der Parteien anzupassen,

\footnotetext{
${ }^{451}$ Risse, NJW 2000, 1614, 1618.

452 Bush/Folger, The Promise of Mediation: Responding to Conflict Through Empowerment and Recognition, S. 16; Boulle/Nesic, Mediation: Principles, Process, Practice, S. 34; Duve, Mediation und Vergleich im Prozess, S. 86.

${ }^{453}$ Bush/Folger, The Promise of Mediation: Responding to Conflict Through Empowerment and Recognition, S. 16.

454 Boulle/Nesic, Mediation: Principles, Process, Practice, S. 382.

455 Risse, Beilage Nr. 9 zu BB 1999, 1, 2.

456 Boulle/Nesic, Mediation: Principles, Process, Practice, S. 41; Risse, Beilage Nr. 9 zu BB 1999, 1, 2.

${ }^{457}$ Duve, Mediation und Vergleich im Prozess, S. 87.

458 Risse, Beilage Nr. 9 zu BB 1999, S. 1, 2.
} 
kann sich maßgeblich auf die Zufriedenheit der Parteien mit dem Ergebnis der Mediation ${ }^{459}$ auswirken. ${ }^{460}$

Andererseits ist mit Blick auf die Qualität des Mediationsergebnisses und die damit verbundene Zufriedenheit der Parteien immer auch an die Gefahr zu denken, dass ein zwischen den Parteien bestehendes Machtungleichgewicht nachteilige Auswirkungen auf die Fairness einer in der Mediation getroffenen Abschlussvereinbarung hat. ${ }^{461}$ In der Mediation fehlen die prozessualen Schutzmechanismen des staatlichen Gerichtsverfahrens, so dass zu befürchten ist, dass eine schwächere Partei durch den stärkeren Gegner in der Mediation übervorteilt wird. ${ }^{462}$ Etwaige Machtungleichgewichte können und sollten in der Mediation bis zu einem gewissen Grad zwar durch den Mediator ausgeglichen werden. ${ }^{463}$ Der Mediator ist jedoch beiden Parteien verantwortlich, so dass zu viel Unterstützung der schwächeren Partei ihn auch in Konflikt mit seiner Rolle als neutraler Dritter bringen kann. ${ }^{464}$

\section{g) Reputation}

In die Überlegungen der Parteien gehen nicht nur Kosten und Nutzen aus einem aktuellen Rechtsstreit ein. So gilt auch für die Partei, dass ihr Verhalten von dem gewünschten öffentlichen Bild und der Reputation beeinflusst wird. So wird z.B. ein Beklagter, der sich den Ruf eines unnachgiebigen Gegners aneignen will, aus einem wenig kompromissbereit geführten Rechtsstreit einen zusätzlichen Nutzen ziehen $^{465}$ und sich daher auch gegen die gerichtsnahe Mediation entscheiden. Um-

459 Als alleiniger Bewertungsmaßstab für die Beurteilung der Qualität einer in der Mediation entwickelten Konfliktlösung ist die Zufriedenheit der Parteien allerdings wohl nicht geeignet. Vgl. insoweit Breidenbach, Mediation: Struktur, Chancen und Risiken von Vermittlung im Konflikt, S. 191; Luban, 66 Denver University Law Review 381, 404 f. (1989); Tyler, 66 Denver University Law Review 419, 432 (1989), die darauf hinweisen, dass die Zufriedenheit der Parteien mit dem Mediationsergebnis auch lediglich auf geringe Ausgangserwartungen der Parteien zurückzuführen sein kann.

460 Bush/Folger, The Promise of Mediation: Responding to Conflict Through Empowerment and Recognition, S. 16.

461 Breidenbach, Mediation: Struktur, Chancen und Risiken von Vermittlung im Konflikt, S. 174 ff.; Coyle, 36 Osgoode Hall Law Journal 625, 642 ff. (1998).

462 Coyle, 36 Osgoode Hall Law Journal 625, 647 (1998); Fiss, 93 Yale Law Journal 1073, 1076 ff. (1984).

463 Breidenbach, Mediation: Struktur, Chancen und Risiken von Vermittlung im Konflikt, S. 231; Coyle, 36 Osgoode Hall Law Journal 625, 647 (1998); Davis/Salem, 6 Mediation Quaterly 17, 18 ff. (1984); Mayer, 16 Mediation Quaterly 75, 80 f. (1987).

${ }^{464}$ Luban, 66 Denver University Law Review 381 ff. (1989).

465 Cooter/Rubinfeld, 27 Journal of Economic Literature 1067, 1074 f. (1989). 
gekehrt gilt für einen Anspruchsgegner, der in der Öffentlichkeit keine Reputationseinbuße erleiden will, dass er eine nicht öffentlich geführte Verhandlung und eine konsensuale Lösung vorziehen wird.

\section{h) Obligatorische Mediation}

360 Fraglich ist schließlich, ob in Deutschland die Einführung einer obligatorischen, mit anderen Worten einer zwangsweisen, Mediation vor dem eigentlichen streitigen Gerichtsverfahren sinnvoll sein könnte:

361 Vordergründig betracht liegt es bereits aus Kostengesichtspunkten durchaus nahe, an eine obligatorische Mediation zu denken. Legt man die PEBB $\mathbb{S}$-analoge Untersuchung zugrunde, so stellt man fest, dass es für die Erfolgswahrscheinlichkeit der Akquisen belanglos ist, ob zunächst eine Untersuchung der Fälle auf ihre Mediationsgeeignetheit erfolgt oder ob wahllos Fälle in die Mediation abgegeben werden. ${ }^{466}$ So liegt die Erfolgswahrscheinlichkeit für die Akquisen ohne vorherige Beurteilung einer möglichen Eignung bei 68,75\%, während sie bei einer vorgenommen Beurteilung bei lediglich 29,17\% liegt. Auch liegt die Erfolgswahrscheinlichkeit bei den ohne Eignungsprüfung durchgeführten Mediationen - lediglich $11,11 \%$ der Mediationen endeten ohne ein Ergebnis - so hoch, dass sich hieraus keine Relativierung ergibt. Man könnte hieraus den Schluss ziehen, dass es ohne Belang sei, ob sich zuvor jemand über die Mediationsgeeignetheit Gedanken gemacht hat, da, wie das Göttinger Modell zeigt, auch ohne vorherige Bewertung gute, ja sogar noch bessere, Erfolgsquoten erzielt werden, als dies bei einer vorherigen Überprüfung auf eine Eignung hin der Fall ist. Konsequenz aus diesem Gedankenansatz wäre, dass die der Justiz entstehenden Kosten durch die Einführung einer obligatorischen Mediation drastisch gesenkt werden könnten, da zum einen die Verfahrensdauer in der Mediation im Durchschnitt wesentlich geringer ausfällt und zum anderen zu großen Teilen langwierige Beweiserhebungen durch die Mediation eingespart werden können.

362 Ein solcher Ansatz verkennt jedoch, dass, selbst wenn man die beste Erfolgsquote bei den Akquisen zugrunde legt, immerhin 31,25\% nicht an einer Mediation teilnehmen wollten. Demgemäß ist ein substantieller Teil der Parteien nicht bereit, an einer Medaition konstruktiv mitzuwirken, was zwangsläufig zu einem Scheitern der Mediation führt, da die Mediation auf diese Mitwirken der Parteien angewiesen ist. Daraus resultieren zwei Konsequenzen:

\footnotetext{
466 Siehe hierzu „Abschlussbericht zur Ausweitung des Begleitforschungsprojekts ,Gerichtsnahe Mediation in Niedersachen', S. 22, erscheint demnächst.
} 
Zum einen wird das Vertrauen in die bzw. die Zufriedenheit mit der Justiz Schaden nehmen, weil die Parteien sich unverstanden fühlen. Eine Partei, die ihren Rechtsstreit durch eine gerichtliche Entscheidung beendet wissen möchte, gerade weil sie die autoritäre Entscheidung des Richters herbeiführen möchte und die sich gegen den Weg der Mediation entschieden hätte, wird nicht verstehen können, warum sie sich mit der anderen Partei zusammensetzen und noch einmal in den Dialog mit ihr treten sollte.

Zum anderen könnte der Prozess durch diese Vorgehensweise künstlich in die Länge gezogen werden, wodurch noch mehr Kosten entstehen könnten, als dies der Fall gewesen wäre, wären die Parteien gleich zu Beginn in das streitige Verfahren eingetreten. ${ }^{467}$ Dies mag zunächst im Widerspruch zu der PEBB $\ Y$-analogen Datenauswertung stehen, nach deren Ergebnis die Zeitersparnis nach einer gescheiterten Mediation und dem Wiedereintritt in das streitige Verfahren immer noch 40\% im Vergleich zu einem von Beginn an im Wege des streitigen Verfahrens betriebenen Prozesses beträgt. Doch ging die diesem Wert zugrunde liegende PEBB $\ Y$-analoge Auswertung bei der Berechnung des entsprechenden Erwartungswertes von Wahrscheinlichkeiten aus, die aufgrund einer freiwilligen Teilnahme bzw. Ablehnung einer Mediation durch die Parteien zustande kamen. Um eine genaue Aussage über eine etwaige Kosten- und Zeitersparnis bzw. eine Verlängerung des Prozesses und eine damit einhergehende Steigerung der Kosten treffen zu können, bedürfte es für eine obligatorische Mediation einer weiteren vertieften empirischen Untersuchung.

Dass die Erfolgsquoten im Rahmen einer obligatorischen Mediation jedoch wesentlich geringer liegen, als dies bei einer freiwilligen Mediation der Fall ist, belegen Zahlen aus dem Bereich der britischen Familiengerichtsbarkeit. Hier wurden alle Bürger, die im Rahmen einer Familienstreitigkeit um Prozesskostenhilfe nachsuchten, durch Section 29 des Family Law Act 1996 verpflichtet, zunächst die Möglichkeit eines Mediationsverfahrens in Betracht zu ziehen. Die gewünschte Prozesskostenhilfe erhielten sie erst, wenn ihr Fall als mediationsungeeignet eingestuft wurde oder sie der Durchführung eines Mediationsverfahrens zugestimmt hatten. Die Erfolgswahrscheinlichkeit der auf diese Art und Weise durchgeführten Mediationen liegt bei lediglich 34\%, während es in 29\% der Fälle zu gar keinem Ergebnis, also nicht einmal zu einer Teilvereinbarung, gekommen ist. ${ }^{468} \mathrm{Im} \mathrm{Ge}-$ gensatz dazu liegt die Erfolgswahrscheinlichkeit der nach dem Göttinger Modell durchgeführten Mediationen - also der zwar freiwilligen Mediation, bei der aber

\footnotetext{
467 So Greger, DRiZ 2005, 28, 29.

468 Kilian, FamRZ 2000, 1006, 1008.
} 
nicht auf eine Mediationeignung geachtet wurde - bei 88,15\%, bei einer Misserfolgswahrscheinlichkeit von 11,11\%. ${ }^{469}$ Dies mag kein Ersatz für eine eigene empirische Untersuchung bei einer obligatorischen Mediation in Deutschland sein, aber es legt doch den Schluss nahe, dass die Befürchtungen, eine obligatorische Mediation würde die Verfahren künstliche in die Länge ziehen und den Prozess so kostenintensiver machen, nicht ganz unbegründet sind.

366 Das wohl gewichtigste Argument, das gegen eine obligatorische Mediation spricht, ist jedoch das Selbstverständnis der Mediation selbst. So stützt sich die Mediation auf die „Freiwilligkeit“ der Parteien, miteinander zu reden; der Sinn der Mediation ist es, ein von allen Parteien akzeptiertes Ergebnis zu erreichen. ${ }^{470}$ Wollte man den Parteien hier einen „Zwang zur Mediation“ auferlegen, und nichts anderes stellt eine obligatorische Mediation dar, so würde das System zwar gegebenenfalls noch funktionieren. Der hinter dem Ganzen stehende Sinn würde jedoch ad absurdum geführt werden, da eine Partei, die schon nicht bereit ist, sich in die Mediation zu begeben, sondern dazu gezwungen werden muss, in den meisten Fällen auch keine Akzeptanz für das Ergebnis aufbringen wird. ${ }^{471}$ Insoweit verfängt auch die Argumentation nicht, die darauf abstellt, dass auch bei der obligatorischen Mediation die Freiwilligkeit gewahrt wird: denn die Parteien entscheiden zwar nicht selbst für den Weg der Mediation, können jedoch innerhalb des Mediationsverfahrens den Ablauf und damit auch einen etwaigen Abbruch, der den Wiedereintritt in das streitige Verfahren zur Folge hat, bestimmen. ${ }^{472}$ Auch das Abstellen auf einen potentiellen Abbruch der Mediationsverhandlung und den Eintritt in den streitigen Prozess kann nicht als Kriterium der Freiwilligkeit angesehen werden, da dies den Bürgern schon aufgrund der Rechtswegsgarantie des Art. 20 Abs. 3 GG zugestanden werden muss.

367 Folglich scheidet ein obligatorisches Mediationsverfahren aufgrund des Selbstverständnisses der Mediation von vornherein aus; die Parteien müssen sich vielmehr aus freien Stücken für ein solches Verfahren entscheiden.

\section{i) Fazit}

368 Werden die Anreize der Parteien maßgeblich durch die Kostenrisiken bestimmt, so muss bei einer langfristigen Aufnahme der Mediation in das Leistungsangebot

\footnotetext{
469 siehe hierzu Abschlussbericht PEBB \Y-analoge Auswertung.

470 Stellungnahme des Deutschen Anwaltsvereins, FuR 2003, 354, 355; Löer, ZKM 2005, 183, 184.

471 So auch Kilian, FamRZ 2000, 1006 , 1008; Löer, ZKM 2005, 182, 184; Pitkowits, SchiedsVZ 2005, $81,83$.

472 Greger, ZKM 2004, 196, 198; Mäbler/Mäbler, AnwBl. 1997, 535, 537.
} 
der Justiz der zukünftigen Ausgestaltung der Kostenstrukturen i.w.S. besondere Aufmerksamkeit gewidmet werden, um negative Anreize zu vermeiden und vielmehr positive Anreize für die gerichtsnahe Mediation zu setzen.

Werden langfristig auch für die gerichtsnahe Mediation Gebühren erhoben, unabhängig von der konkreten Ausgestaltung in Form einer Gebührentabelle oder von Stundenhonorarsätzen - so sollten diese (zusammen mit der durch Einleitung des gerichtlichen Verfahrens entstehenden Gerichtsgebühr) jedoch deutlich unter den im Rahmen eines herkömmlichen Gerichtsverfahrens entstehenden Gerichtskosten liegen, um keine negativen Anreize für die Parteien zu setzen. Sollte eine gesetzliche Normierung der gerichtlichen Mediation nicht erfolgen, so sind bei der Ausgestaltung der Gebühren gleichzeitig auch wettbewerbsrechtliche Aspekte stärker zu berücksichtigen und die zu erhebenden Gebühren der Höhe nach denen privatwirtschaftlicher Anbieter anzupassen. ${ }^{473}$

Zur Förderung der gerichtsnahen Mediation ist die Neuregelung der Verteilungsregelung des $₫ 91 \mathrm{ZPO}$ und das Setzen von Kostenanreizen durch Kostensanktionen zu empfehlen. Danach sollte sich die Kostenlastverteilung nicht an dem Prozessausgang orientieren, sondern auch dann einer obsiegenden Partei die Kosten ganz oder zum Teil auferlegen, wenn sie sich einem gerichtlich angeregten Versuch einer einvernehmlichen Konfliktbeilegung ungerechtfertigt widersetzt hat. Zumindest ist hier an eine verpflichtende Teilnahme an einer Informationssitzung zur gerichtsnahen Mediation zu denken.

Soll die gerichtsnahe Mediation sich langfristig als Alternative zum streitigen Verfahren etablieren, wird - unabhängig von dem jeweiligen Kostenmodell - auch die Einführung einer der Prozesskostenhilfe vergleichbaren Mediationskostenhilfe erforderlich sein, damit bestimmte Gruppen nicht von vornherein von der Möglichkeit der Inanspruchnahme der gerichtsnahen Mediation ausgeschlossen werden und die Justiz auch mit Blick auf das Angebot von gerichtsnaher Mediation der Sozialverantwortung des Staates gerecht wird. Darüber könnte auch die Gewährung von Prozesskostenhilfe an das ernsthafte Bemühen um eine einvernehmliche Konfliktbeilegung geknüpft werden.

Regelungsbedarf besteht auch insoweit, als abgesehen von dem für den Mediator bestehenden Zeugnisverweigerungsrecht die Vertraulichkeit des Mediationsverfahrens, insbesondere mit Blick auf einen nachfolgenden Prozess, nur durch Prozessvertrag und auch insoweit nicht vollständig gesichert werden kann. Dies gilt umso mehr im Bereich des Untersuchungsgrundsatzes, in dessen Anwendungsbe-

${ }^{473}$ Siehe hierzu oben Rn. 273. 
reich Beweismittelverträge nicht abgeschlossen werden können. Ein umfassender Schutz der Vertraulichkeit und ein Zeugnisverweigerungsrecht für jeden an der Mediation Beteiligten im nachfolgenden Prozess müsste durch die Einführung eines entsprechenden gesetzlichen Beweisverbotes gewährleistet werden.

373 Zur Klarstellung empfiehlt sich darüber hinaus auch eine Ergänzung des \278 Abs. 5 ZPO mit dem Inhalt, dass ein Richter, der im Rahmen eines gerichtlichen Mediationsangebotes die Mediationsverhandlungen leitet, durch den gesetzlich zuständigen Richter ersucht werden kann, das gerichtliche Verfahren nach Abschluss der Mediation wieder aufzurufen und einen von den Parteien in der Mediation geschlossenen Vergleich zu Protokoll zu nehmen.

374 Ein positives Signal für die Konfliktparteien mit ebenfalls nur klarstellendem Charakter wäre schließlich eine gesetzliche Regelung, die auch für Ausschlussfristen eine Fristhemmung für die Dauer von (Mediations-) Verhandlungen vorsieht.

\section{Anreizstrukturen Rechtsschutzversicherungen}

375 Da die Rechtsschutzversicherungen (RSV) in zahlreichen Fällen letztlich die anfallenden monetären Kosten tragen, ist davon auszugehen, dass diese ebenfalls ein Interesse daran haben, unnötige Kosten zu vermeiden bzw. einen Anreiz haben, kostengünstigere Lösungen zu fördern.

376 Es erscheint möglich, dass die Kosten für die Gerichtsnahe Mediation gegenüber einem herkömmlichen streitigen Verfahren geringer sein können. Insbesondere kommen dabei Kostenersparnisse dadurch in Betracht, dass im Mediationsverfahren meist auf Sachverständige und Zeugen verzichtet wird und daher dann auch im Gegensatz zum streitigen Verfahren diese Kosten nicht erstattet werden müssen. Ebenso ist auch an eine Kostenreduzierung durch die Vermeidung von Folgeprozessen zu denken.

377 Daher ist grundsätzlich davon auszugehen, dass für die Rechtsschutzversicherungen aufgrund von Einsparungsmöglichkeiten ein Anreiz bestehen sollte, gerichtsnahe Mediation zu fördern.

378 Daneben sind auch die positiven Effekte zu bedenken, die eine Aufnahme der (gerichtsnahen) Mediation in das Leistungsprogramm der Rechtsschutzversicherungsanbieter für die Versicherungsnehmer hätte. Einer solchen Erweiterung des Leistungsangebots käme eine Signalwirkung für die Nachfrager auf dem Markt für Konfliktlösungsdienstleistungen zu. 


\section{a) Status quo}

Derzeit ist das Angebot der gerichtsnahen Mediation kostenlos, d.h. es entstehen für die Rechtsschutzversicherungen zusätzlich zu den Prozessgebühren keine weiteren Gebühren. Nach der Einführung des RVG macht es für die Erstattung durch die RSV in gebührenrechtlicher Hinsicht auch keinen Unterschied mehr, ob die Parteien einen herkömmlichen Prozess mit anwaltlicher Vertretung führen oder an einer gerichtsnahen Mediation mit der Unterstützung ihrer Parteianwälte teilnehmen. Auch ohne explizite Aufnahme von Mediationsdienstleistungen in den Leistungskatalog des Versicherungsvertrages sind daher derzeit die im Rahmen der gerichtsnahen Mediation entstehenden Kosten von der Erstattung der RSV gedeckt. Insofern bestehen hier nach der Einführung des RVG keine negativen Anreize mehr. ${ }^{474}$

Für den Versicherungsnehmer bewirkt das derzeitige Verhalten der Rechtsschutzversicherungen einen eindeutigen Kostenvorteil der gerichtsnahen Mediation gegenüber anderen außergerichtlichen Streitschlichtungsangeboten, deren Kosten oft nicht von den Rechtsschutzversicherungen übernommen werden. ${ }^{475}$

\section{b) Ausblick: Angebot eines Versicherungsschutzes für (gerichtsnahe) Mediation und mögliche Probleme}

\section{aa) Grundsätzliche Überlegungen zu einem Versicherungsschutz für (gerichtsnahe) Mediation}

Stellt sich gerichtsnahe Mediation tatsächlich in bestimmten Fällen auch für die Rechtsschutzversicherungen als kostengünstigere Lösung und auch als Möglichkeit zur Einsparung von durch Folgeprozessen verursachten Kosten dar, erscheint es somit langfristig angebracht, auch einen Versicherungsschutz für die (gerichtsnahe) Mediation anzubieten, um so einen zusätzlichen Anreiz für den Versicherungsnehmer zu setzen, eine kostengünstigere Lösung zu wählen.

Werden für die Inanspruchnahme der gerichtsnahen Mediation langfristig gesonderte Gebühren erhoben, so ist bei einer Erstattung durch die RSV zu bedenken, dass sich insbesondere im Fall des Scheiterns der Mediation und der Fortsetzung des streitigen Verfahrens die von der Rechtsschutzversicherung zu erstattenden

\footnotetext{
474 Zu Überlegungen nach der Rechtslage nach der BRAGO siehe Spindler/Apel/Spalckhaver, ZKM 2003, 192, $196 \mathrm{f}$.

475 Hier oben Rn. 307.
} 
Kosten in einigen Fällen auch erhöhen könnten. Zunächst wäre mit Blick auf die Gefahr des Scheiterns der gerichtsnahen Mediation zumindest daran zu denken, die Kosten für die anwaltliche Begleitung bis zu den Anwaltsgebühren in einem Prozess erster Instanz bei erfolgreicher Mediation auch von der RSV zu erstatten lassen. Dann würde der Versicherungsnehmer bei gleich bleibender Prämienzahlung zumindest dann gleichgestellt, wenn die gerichtsnahe Mediation den herkömmlichen Prozess ersetzt und keine zusätzlichen Kosten verursacht. Dafür spricht auch, dass in einem Schiedsverfahren auch die Kosten bis zur Höhe der in einem Prozess erster Instanz entstehenden Anwaltsgebühren von den Rechtsschutzversicherungen erstattet werden. 476

383 Allerdings ist auch von einem Sinken der von der RSV zu erstattenden Kosten auszugehen, da zum einen - wie bereits angesprochen - die Kosten für Zeugen und Sachverständigen durch die Durchführung von gerichtsnaher Mediation reduziert werden, zum anderen langfristig auch an eine Kostenreduzierung durch die Vermeidung von Folgeprozessen zu denken ist.

384 Grundsätzlich ist eine Erstreckung des Versicherungsschutzes auf die gerichtsnahe Mediation denkbar. Ein Versicherungsschutz wird dann nachgefragt, wenn es im menschlichen Leben unvorhergesehene und einzelwirtschaftlich auch unvorhersehbare Ereignisse gibt, die den Betroffenen oder andere Personen mit erheblichen Aufwendungen belasten und/oder zu Einnahmeausfällen führen. Es besteht daher ein Bedarf, die Aufwendungen oder Einnahmeausfälle in solchen Fällen ersetzt zu bekommen und damit den Nutzen unter Unsicherheit des Eintreffens zukünftiger Ereignisse zu maximieren. Aufgrund der Unsicherheiten dieser Ereignisse besteht eine Nachfrage für Versicherungsdienstleistungen. Der Nachfrager verpflichtet sich dann, in regelmäßigen Abständen Beträge bzw. Prämien zu entrichten. Als Gegenleistung verpflichtet sich der Versicherer, bei Eintritt des Versicherungsfalles dem Betroffenen die Aufwendungen zur Beseitigung des Schadens zu ersetzen oder sonst Zahlungen zu leisten. Das einzelwirtschaftliche Risiko wird bei einer größeren Zahl von Beteiligten kalkulierbar und auf alle Versicherungsnehmer verteilt. Kalkulierbar heißt, dass ein Risiko versicherbar ist, wenn der Versicherer wegen der Vielzahl von Fällen, mit denen er es zu tun hat, eine bessere Prognose über die infolge bestimmter (Schadens-)Ereignisse auf ihn zukommenden Zahlungsverpflichtungen abgeben kann als der mit dem Einzelfall konfrontierte Versicherungsnehmer. ${ }^{477}$ Die Nachfrage nach einem Versicherungsschutz besteht daher auch für die gerichtsnahe Mediation. Wie auch im Falle der klassi-

\footnotetext{
${ }^{476}$ So Mitteilung $W$. Brundiek v. 20. November 2002 abrufbar unter: http://www.centrale-fuermediation.de/info.htm.

477 Siehe dazu Stobbe, Mikroökonomik, S. 466 f.
} 
schen Rechtsschutzversicherung sind die durch eine gerichtsnahe Mediation entstehenden Kosten auch von dem nicht vorhersehbaren Eintritt eines Rechtsstreits abhängig. Ebenso ist auch die Höhe der durch die gerichtsnahe Mediation entstehenden Kostenbelastung nicht vorhersehbar. Auch hier besteht ein Bedürfnis seitens der potentiellen Parteien eines Rechtsstreits, die unsicheren Kosten für die Streitbeilegung in eine kalkulierbare Belastung durch eine Prämienzahlung umzuwandeln.

Ohne die konkrete Gestaltung einer späteren Gebührenordnung für die gerichtsnahe Mediation können allerdings kaum konkrete Aussagen über die insgesamt von den Rechtsschutzversicherungen zu tätigenden Ausgaben getroffen werden.

Grundsätzlich gilt es aber, bei der späteren Ausgestaltung eines Versicherungsschutzes für die (gerichtsnahe) Mediation folgende Probleme und spezielle Gefahren von Marktstörungen zu beachten.

\section{bb) Probleme}

\section{(1) Moral hazard/moralisches Wagnis für die Versicherer}

Zunächst bestehen im Rahmen von Versicherungsgeschäften spezielle Gefahren von Marktstörungen dergestalt, dass Versicherungsnehmer den Eintritt des Versicherungsfalls begünstigen können, dass Versicherungsverträge überwiegend von solchen Nachfragern nach Versicherungsleistungen abgeschlossen werden, deren Risiko höher ist als das mittlere Risiko der Personengruppe, dass der Versicherer der Betragsberechnung zugrunde legt oder dass Versicherungsnehmer ihren Schaden höher als tatsächlich eingetreten bewerten oder angeben. ${ }^{478}$ Aus der Sicht des Versicherers sind diese Gefahren als moralisches Wagnis (moral hazard) zu bezeichnen, die nicht zutreffend eingeschätzt werden können. In diesen Fällen besteht auf dem Markt für Versicherungsleistungen ein Informationsproblem: Der Versicherer ist außerstande, in jedem Einzelfall mit genügender Sicherheit und zu erträglichen Kosten festzustellen, ob die Neuzugänge zum Versichertenkollektiv nicht systematisch mit überdurchschnittlichem Risiko behaftet sind und ob die unter Mitwirkung der Versicherungsnehmer zustande gekommenen Schadensereignisse dem Eintritt und der Höhe nach unvermeidlich waren. Der Versicherer ist also in Bezug auf Neuzugänge, Schadenseintritt und -höhe marginal von der Willkür seiner Vertragspartner abhängig. ${ }^{479}$

\footnotetext{
478 Siehe dazu Stobbe, Mikroökonomik, S. 466 f.

479 Stobbe, Mikroökonomik, S. 469.
} 
388 Auch bei einem Versicherungsschutz für gerichtsnahe Mediation bestünde für den Versicherer ein solches moralisches Wagnis. Auch hier ist die Beziehung durch eine Informationsasymmetrie gekennzeichnet: Die Versicherer tragen zwar letztlich die Kosten, doch haben sie auf den Erfolg der Mediation und auf die durch ein evtl. Scheitern der Mediation zusätzlich entstehende Kosten keinen Einfluss, sondern sind von den Handlungen und auch der Vergleichsbereitschaft ihrer Mandanten abhängig. Die RSV kann das Verhalten des Versicherungsnehmers, ob er evtl. ein Scheitern der Mediationsverhandlungen und somit das Entstehen zusätzlicher Kosten (eines Prozesses) bewusst verursacht hat, nicht beurteilen. Insbesondere kann dieses Problem entstehen, wenn der Versicherungsnehmer die gerichtsnahe Mediation lediglich missbraucht, um Informationen über die gegnerische Partei zu gewinnen, aber an einer konsensualen Streitbeilegung nicht wirklich interessiert ist.

\section{(2) Adverse Selection/Negativauslese}

389 Auch wäre eine Versicherungsleistung für (gerichtsnahe) Mediation von einer herkömmlichen RSV aufgrund der bestehenden Unterschiede zwischen den beiden Arten der Streitbeilegung zu trennen. Ein einheitlicher (nicht-individueller) Versicherungsschutz für alle Versicherungsnehmer für (gerichtsnahe) Mediation und die klassische RSV könnte zu einer Negativauslese (adverse selection) führen.

390 Das Problem der Negativauslese besteht immer dann, wenn zwar die (potentiellen) Versicherungsnehmer ihr eigenes Versicherungsrisiko einschätzen können, die Versicherer hingegen nicht. Die Versicherungsverträge sind dann immer weniger effizient, als sie es wären, wenn eine individuell auf die Bedürfnisse des jeweiligen Versicherungsnehmers abgestimmte Versicherungspolice angeboten werden könnte. ${ }^{480}$

391 Diese Gefahr einer Negativauslese besteht auch bei einem Versicherungsschutz für die Kosten der gerichtsnahen Mediation. Diese Gefahr der unterschiedlichen Risiken, des unterschiedlichen Nutzens und der unterschiedlichen Erwartungen der potentiellen Versicherungsteilnehmer, die in den Unterschieden des normalen streitigen Verfahrens und der gerichtsnahen Mediation begründet sind, sprechen gegen eine gemeinsame Versicherung der gerichtsnahen Mediation und des normalen Prozessrisikos. Würde langfristig eine Einbeziehung der gerichtsnahen Me-

${ }^{480}$ Rothschild/Stiglity, 90 Quarterly Journal of Economics 629 - 649 (1976); Layard/Walters, Microeconomic Theory, 1978, S. 384; Stobbe, Mikroökonomik, S. 486 f.; grundlegend zu dem Problem der Negativauslese Akerloff, 84 Quarterly Journal of Economics 488 - 500 (1970); vgl. auch Richter/Furubotn, Neue Institutionenökonomik, S. 165. 
diation in den normalen Versicherungsschutz der klassischen Rechtsschutzversicherung erfolgen, so würden dann Personen eine solche Versicherung abschlieBen, die gar kein Interesse an einer konsensualen Streitbeilegung haben. Diese könnten die Kosten nur unnötig in die Höhe treiben, indem sie - wenn ohnehin auch die Kosten für die gerichtsnahe Mediation vom Versicherungsschutz umfasst wären - die gerichtsnahe Mediation zwar zunächst einleiten (da sie ohnehin vom Versicherungsschutz gedeckt wäre), dann aber aufgrund mangelnden Interesses an einer konsensualen Streitbeilegung ein Scheitern der Mediation und somit das Entstehen zusätzlicher Kosten verursachen würden. Dann bestünde die Gefahr der adverse selection (Negativauslese).

Für eine Trennung des Verischerungsschutzes könnten weiterhin Unterschiede zwischen dem streitigen Verfahren und der Mediation sprechen, die sich auch auf den unterschiedlichen Nutzen der Versicherten ergeben. Im Rahmen der Mediation entsteht i.d.R. ein zusätzlicher positiver immaterieller Nutzen (z.B. durch Sicherung langfristiger Beziehungen, höhere Zufriedenheit, schnelleres Erlangen eines Titels, Senkung der oben genannten Transaktionskosten), der aber nicht Inhalt des normalen Rechtsschutzversicherungsvertrages ist. Dieser zielt nämlich nur auf die Versicherung gegen das Risiko der Kostenlast im Prozess ab. Es erscheint daher als volkswirtschaftlich sinnvolle Lösung, die dem zusätzlichen positiven Nutzen entsprechenden zusätzlichen Kosten auch dort zu internalisieren, wo sie entstehen, d.h. bei den Parteien. Dieser Nutzen ist aber von der individuellen Präferenzordnung des Versicherungsnehmers abhängig, insbesondere davon, wie viel Nutzen einer konsensualen Streitbeilegung beigemessen wird. Von der jeweiligen individuellen Präferenzordnung ist auch die Zahlungsbereitschaft für eine zusätzliche Versicherungsprämie abhängig.

$\mathrm{Zu}$ den versicherungsrelevanten Unterschieden zwischen dem normalen streitigen Verfahren und der gerichtsnahen Mediation zählt auch, dass der klassischen Rechtsschutzversicherung neben der normalen Versicherungsfunktion vor allem auch eine Funktion als Drohsystem zukommt, das seinen Besitzern seine entscheidenden Erträge durch die Schädigung bedrohter Dritte liefert. Danach besteht der entscheidende Vorteil, den eine Rechtsschutzversicherung ihren Mitgliedern zu bieten vermag, nicht in den Nutzengewinnen, die mit einer Verminderung der Schwankungsmöglichkeiten des zukünftigen Einkommens durch zukünftige unsichere Ereignisse verbunden sind, sondern darin, dass außen stehende Dritte in Verhandlungen durch den Hinweis auf die grenzkostenfreie Prozessführungsmöglichkeit bedroht werden können und damit zu Zugeständnissen genötigt werden, 
die diese andernfalls nicht gemacht hätten. ${ }^{481}$ Eine solche Funktion einer Versicherung widerspricht aber dem Wesen der Freiwilligkeit der gerichtsnahen Mediation.

\section{cc) Empfohlene Ausgestaltungsmöglichkeiten eines Versicherungsschutzes}

394 Auch wenn die nähere Ausgestaltung von vielen - zum Teil noch unbekannten Faktoren abhängig ist, deren Auswirkungen und Zusammenspiel an dieser Stelle nicht abschließend geklärt werden können, sollen an dieser Stelle gleichwohl einige mögliche Ausgestaltungen eines Versicherungsschutzes für (gerichtsnahe) Mediation aufgezeigt werden.

395 Zum einen ist aufgrund des zuletzt beschriebenen Problems der Negativauslese ein von der herkömmlichen RSV getrennter Versicherungsschutz für die (gerichtsnahe) Mediation empfehlenswert. Auch ist zu bedenken, dass das Angebot unterschiedlicher RSV-Verträge eine Selbstselektion der Versicherungsnehmer entsprechend ihrer eigenen Präferenzen ermöglich kann.

396 Da die Versicherung nicht in der Lage ist, die Qualitäten und die Absichten ihrer Versicherungsnehmer zutreffend einzuschätzen, und um das Moral HazardProblem für die Versicherungen abzuschwächen, ist auch für die (gerichtsnahe) Mediation - wie für die klassische Rechtsschutzversicherung - an eine Selbstbeteiligung oder eine Co-Insurance (Versicherungsnehmer zahlt immer einen bestimmten Prozentsatz der entstehenden Kosten) ${ }^{482}$ zu denken . Ein Überwachungssystem, um ex post ein offensichtliches bewusstes Verursachen des Scheiterns der Mediation durch eine Partei zu erkennen und zu sanktionieren, ist wenig viel versprechend aufgrund der Schwierigkeit der Bewertung.

397 Die Möglichkeit, durch die Inanspruchnahme von alternativen Streitlösungsmechanismen und somit auch der (gerichtsnahen) Mediation die Kosten für die RSV zu senken, sollte von den RSV nicht ungenutzt bleiben. So erscheint auch das Setzen positiver Anreize zur Mediation durch die Ausgestaltung der RSV-Verträge denkbar und sinnvoll. Denkbar ist hier z.B. auch ein Kostenanreiz durch eine geringere Selbstbeteiligung an den Kosten der Konfliktlösung bei erfolgreicher Mediation.

\footnotetext{
481 Adams, Ökonomische Analyse des Zivilprozesses, S. 370.

482 Layard/Walters, Microeconomic Theory, 1978, S. 384; Stobbe, Mikroökonomik, S. 484.
} 
Darüber hinaus sollte die RSV durch die Ausgestaltung ihrer Versicherungsverträ398 ge auch Qualitätserfordernissen Rechnung tragen und die Erstattungsfähigkeit der Kosten von der Wahl eines einen gewissen Qualitätsstandard gewährleistenden Mediators (Selbstverpflichtung; Gütesiegel etc.) eingrenzen. 


\section{E. Dritter Teil: Die empirischen Ergebnisse}

\section{I. Überblick}

399 Die Akzeptanz der Beteiligten ist entscheidend für den Erfolg der gerichtsnahen Mediation, damit diese eine tatsächliche Alternative zum herkömmlichen streitigen Gerichtsverfahren darstellen kann. Die Begleitforschung untersuchte hierfür die rechtlichen Rahmenbedingungen auf ihre Auswirkungen und ihre effiziente Ausgestaltung. Im Wege der empirisch-quantitativen und -qualitativen Methode wurden die Motivationen der Verfahrensbeteiligten ermittelt, analysiert und die zuvor theoretisch abgeleiteten Annahmen dem empirischen Test unterworfen. Aus methodischer Sicht sind allerdings einige Einschränkungen zu berücksichtigen, die finanziellen Restriktionen geschuldet waren: So wären an sich Vergleichsgerichte, an denen keine Mediation durchgeführt wird, heranzuziehen, um die Wirkungskraft der gerichtsinternen Mediation abzuschätzen. Auch bedürfte es längerfristiger Begleitung der mediierten Parteien, um tatsächlich sicher den dauerhaften Befriedigungseffekt einzuschätzen. Schließlich müsste im Sinne eines Institutionenvergleichs auch die Situation untersucht werden, in der alternative Streitschlichter, etwa vom Gericht beauftragte Anwaltsmediatoren die Mediation durchführen. Die nachfolgende Darstellung stützt sich dabei auf die Erhebungen und Auswertung der Ergebnisse einer quantitativen und qualitativen Erhebung, die vom sozialwissenschaftlichen Institut arpos im Rahmen des Forschungsprojektes durchgeführt wurde - für die dabei angewandten Methoden sowie für die weiteren Ergebnisse muss hier auf die entsprechenden Publikationen des arpos-Instituts verwiesen werden. ${ }^{483}$

\section{Anreizstrukturen Richterschaft}

400 Eine der Gruppen, die an der gerichtsinternen Mediation beteiligt sind, ist die Richterschaft - zum einen in ihrer Rolle als Fallmanager, zum anderen als Mediator. Im Folgenden soll untersucht werden, ob sich die im Rahmen der ökonomischen Analyse des Rechts getroffenen Annahmen im Hinblick auf diese Beteiligten als zutreffend herausgestellt haben:

${ }^{483}$ Eine Zusammenfassung findet sich unter http://www.arpos.de/download/fb5.pdf. 


\section{Richter als Fallmanager}

\section{a) Arbeitszeitersparnis}

\section{aa) Konzentration auf wesentliche streitige Verfahren}

Die Annahme, dass durch die Abgabe mediationsgeeigneter Fälle in die Mediation für den Fallmanager die Möglichkeit besteht, sich auf die wesentlichen und rechtlich anspruchsvollen Fälle zu konzentrieren, lässt sich durch die empirische Datenerhebung vorsichtig bestätigen. So stimmten dieser Aussage 38,3\%484 der insgesamt 94 befragten Richter zu. Demgegenüber stehen 23,4\% ${ }^{485}$ der Richter, die dem nicht zustimmen können. Während 6,4\% der Befragten keine Angabe zu der entsprechenden Frage machten, waren 31,9\% der befragten Richter indifferent, so dass sie der Aussage weder zustimmen, noch diese ablehnen konnten.

Auch eine Aufschlüsselung in Zivil- und Sozialgerichtsbarkeit ${ }^{486}$ ergibt ein ähnliches Ergebnis. Während bei den Zivilgerichten 31,65\% der befragten Richter der Möglichkeit, sich durch die Abgabe von Fällen in die Mediation auf wesentliche und rechtliche Anspruchsvolle Fälle beschränken zu können, indifferent gegenüber stehen, lehnen 24,05\% ${ }^{487}$ diese Aussage ab. Während sich 7,59\% der insgesamt 79 der Zivilgerichtsbarkeit zuzuordnenden Richter sich nicht zu der Frage äußerten, stimmten 36,71\% der Aussage zu.

Bei den Sozialgerichten sprachen sich von den insgesamt zwölf befragten Richtern 16,66\% ${ }^{488}$ gegen die getroffene Aussage der Möglichkeit der Konzentration auf wesentliche und wichtige Fälle aus. Genau ein Drittel, also 33,33\%, konnte dieser Aussage weder zustimmen noch diese ablehnen, während die übrigen 50,0\% der Ansicht waren, sich auf die wichtigen Fälle beschränken zu können.

Insoweit scheint es zumindest schon unter dem Gesichtspunkt der möglichen Konzentration der Richterschaft auf die wesentlichen streitigen Fälle wünschenswert, die gerichtsinterne Mediation in Niedersachsen zu integrieren, um so zumindest eine gewisse Entlastung bei der Richterschaft zu erreichen.

\footnotetext{
484 16,0\% ,stimme vollkommen zu“; 22,3\% ,stimme eher zu“.

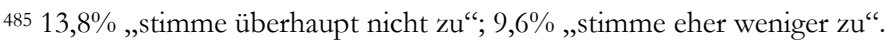

486 Eine Auflistung der Verwaltungsgerichte erübrigte sich, da lediglich ein Fragebogen vorliegt; um eine aussagekräftigere Statistik zu bekommen, wurden Zivil- und Familiengerichtsbarkeit zusammengefasst.

487 13,92\% „stimme überhaupt nicht zu“; 10,13\% „stimme eher weniger zu“.

488 8,33\% „stimme überhaupt nicht zu“; 8,33\% „stimme eher weniger zu“.
} 


\section{bb) Reduzierung des Arbeitsaufwandes}

405 Mit der Möglichkeit der Ersparung von Arbeitsaufwand bzw. der Möglichkeit zu selektieren, welche Fälle in die Mediation abgegeben werden, geht auch immer die Gefahr einher, dass die Fallmanager versucht sein könnten, sich unliebsamer und langwieriger Fälle dadurch zu entledigen, dass gerade diese Fälle vermehrt in die Mediation gegeben werden. Gleichzeitig wäre damit eine Behinderung der Mediation verbunden, da die Fälle nur noch nach persönlichem Vorteil und nicht mehr nach Geeignetheit abgegeben würden.

406 Im Rahmen des Modellprojekts ergeben sich jedoch aus den Fragebögen keine Hinweise, dass lediglich unliebsame und langwierige Fälle in die Mediation gegeben wurden. Von den insgesamt 274 - wobei hier zu berücksichtigen ist, dass mit 177 der Großteil der beantworteten Fragebögen aus der Zivilgerichtsbarkeit stammen - enthielten 268 eine Antwort auf die Frage der Gesamtkomplexität des Falles. Dabei schätzten 52,2\% der Mediatoren die Komplexität als mittel ein. Die übrigen knapp 50\% verteilen sich dann nahezu gleichmäßig auf eine geringe $(25,0 \%)$ sowie auf eine hohe Komplexität (22,8\%), sodass man hier wohl von einer gewöhnlichen Abgabe in die Mediation wird ausgehen können; Hinweise auf einen Missbrauch der Mediation zur persönlichen Vorteilgewinnung der Fallmanager ergeben sich hier nicht.

407 Bei Aufschlüsselung der gewonnenen Daten nach den einzelnen Gerichtsbarkeiten ergibt sich ein leicht verändertes Bild: Gemein ist allen Gerichtsbarkeiten, dass die Gesamtkomplexität der in die Mediation abgegeben Fälle durch die Mediatoren überwiegend als mittel eingestuft wird. Die Angaben reichen hier von den Zivilgerichten mit 47,46\% als niedrigstem, bis zu 64,81\% bei den Sozialgerichten als höchstem Ergebnis. Die Familien- und Verwaltungsgerichte reihen sich hier mit 53,33\% bzw. 59,09\% im statistischen Mittelfeld ein. Allerdings ist bei diesen beiden letztgenannten Gerichtsbarkeiten zu bedenken, dass diese Angaben allenfalls als vorsichtige Tendenz bezeichnet werden können, da die Gesamtzahl der beantworteten Fragebögen (15 bei den Familien- und 22 bei den Verwaltungsgerichten) zu niedrig ist, als dass sich hieraus aussagekräftige Ergebnisse gewinnen ließen.

408 Änderungen zum Ergebnis der Gesamtschau aller Gerichtsbarkeiten nach der Frage der Komplexität des Falles ergeben sich jedoch für die Einstufung einer geringen oder hohen Komplexität. Während bei den Zivilgerichten die übrigen Fälle überwiegend als wenig schwierig eingestuft wurden (32,2\% zu 20,34\%), kehrt sich dieses Ergebnis bei den übrigen Gerichtsbarkeiten um: Hier wurde zum Teil ein deutliches Übergewicht der Abgabe komplexer Fälle im Verhältnis zu einfachen Fällen angenommen. Bei den Sozialgerichten stellt sich dieses Verhält- 
nis als 22,22\% zu 12,96\%, bei den Familiengerichten 33,33\% zu 13,33\% und bei Verwaltungsgerichten sogar als 36,36 zu 4,55\% dar.

Da es aber auch bei diesen beiden Gerichtsbarkeiten zumeist Fälle mittlerer Komplexität sind, die in die Mediation abgegeben werden, lassen sich auch hier die Bedenken eines möglichen Missbrauchs der Mediation zur persönlichen Entlastung des Fallmanagers nicht bestätigen.

Wie schon die statistische Erhebung im Zusammenhang mit der Komplexität der in die Mediation abgegebenen Fälle deutet auch die Frage nach den Beweisschwierigkeiten darauf hin, dass die gerichtsnahe Mediation nicht dazu missbraucht wird, sich unliebsamer Fälle zu entledigen, sondern dass es sich hierbei vielmehr um eine im Vergleich zum streitigen Gerichtsverfahren gleichberechtigte Alternative handelt .

Auch hier wurde nicht in allen 274, sondern lediglich in 265 Fällen auf die Frage hinsichtlich etwaiger Beweisschwierigkeiten eingegangen. Ebenso wie im Zusammenhang mit der Fragestellung nach der Komplexität des Falles schätzte auch hier wieder der Großteil der befragten Mediatoren die Beweisschwierigkeit als „mittel“ ein; hiervon gingen 44,2\% der 265 gültigen Antworten aus. Die übrigen 52,6\% verteilen sich dann paritätisch auf die Angabe, dass es geringe bzw. hohe Beweisschwierigkeiten gab.

Bemerkenswert erscheint jedoch, dass die Ergebnisse der einzelnen Gerichtsbarkeiten kein einheitliches Bild abgeben. Bei den Zivil- und Sozialgerichten - den beiden Gerichtsbarkeiten mit der höchsten Rücklaufquote ${ }^{489}$ - stufte der überwiegende Teil der Mediatoren, wie schon bei der Gesamtbetrachtung, die Beweisschwierigkeiten als mittel ein, nämlich 48,35\% bzw. 51,85\%. Die Anzahl der Fälle, die mit geringen und mit hohen Beweisschwierigkeiten einhergehen, verhält sich dann jedoch genau gegensätzlich zueinander. Während bei der Zivilgerichtsbarkeit $29,12 \%$ die Beweisschwierigkeiten als hoch ansahen, waren es nun 18,68\%, die sie als gering einstuften. Anders hingegen bei den Sozialgerichten: Hier waren es $27,78 \%$, die die Beweisschwierigkeiten als gering beurteilten, während 20,37\% sie als hoch einstuften.

Völlig aus dem Rahmen fallen indes die Familien- und Verwaltungsgerichte, wobei auch an dieser Stelle auf die geringe Gesamtzahl der zurückgesandten Fragebögen $^{490}$ und der damit einhergehenden geringen Aussagekraft der Zahlen hingewie-

489 Zivilgerichte 182 - wobei sieben diese Frage nicht beantwortet haben; Sozialgerichte 54.

49016 von den Familiengerichten, wovon schon 2 keine Aussage über die Beweisschwierigkeiten enthielten sowie 22 von den Verwaltungsgerichten. 
sen werden soll. Daher kann allenfalls eine vorsichtige Tendenz, aber keine statistisch gesicherte Aussage getroffen werden. Bei den beiden genannten Gerichtsbarkeiten sehen die befragten Mediatoren die Beweisschwierigkeiten in den vorliegenden Fällen zum überwiegenden Teil als gering an; bei den Familiengerichten sind dies 62,5\% und bei den Verwaltungsgerichten 59,09\%. Ob die übrigen Fälle dann eine mittlere oder hohe Beweisschwierigkeit aufweisen, wurde von den beiden Gerichtszweigen unterschiedlich beurteilt. Während an den Familiengerichten immerhin noch 18,75\% von einer mittleren Beweisschwierigkeit und nur 6,25\% von einer hohen ausgingen, waren es bei den Verwaltungsgerichten lediglich 9,09\% die die Beweisschwierigkeiten als mittel einstuften, 31,82\% jedoch als hoch.

414 Zusammenfassend lässt sich festhalten, dass sich nach der statistischen Auswertung die in der ökonomischen Analyse aufgestellte Befürchtung, dass gegebenenfalls überwiegend unliebsame und langwierige Fälle und somit solche, bei denen mit immensen Beweisschwierigkeiten zu rechnen ist, in die Mediation gegeben werden, nicht bewahrheitet hat. Vielmehr wurden die Beweisschwierigkeiten überwiegend „mittel“ oder sogar nur als „gering“ eingestuft, was darauf hindeutet, dass die Langwierigkeit oder Unliebsamkeit bestimmter Fälle kein Auswahlkriterium war, sondern vielmehr im Hinblick auf die Beweiserhebung durchschnittliche Fälle in die Mediation abgegeben wurden.

415 Ein weiteres Indiz dafür, dass sich die Befürchtungen, nur schwierige und unliebsame Fälle würden in die Mediation abgegeben, nicht bestätigt, liefert die Untersuchung hinsichtlich der Schwierigkeit der rechtlichen Probleme der in Rede stehenden Fälle. ${ }^{491}$

416 Auch, wenn sich die im Rahmen der ökonomischen Analyse angesprochenen Befürchtungen nicht bewahrheitet haben, so gibt es dennoch Möglichkeiten, einem etwaigen Missbrauch der Mediation, der sich unter Umständen zu einem späteren Zeitpunkt, sollte die gerichtsnahe Mediation niedersachsenweit in das Justizangebot aufgenommen werden, einstellen könnte, entgegenzuwirken. Hierfür bieten sich zwei Lösungsalternativen an. Die erste Möglichkeit wäre eine gesetzlich vorgeschriebene Durchführung einer Mediation, unabhängig davon, um welche Gerichtsbarkeit es sich handelt und unabhängig davon, ob der jeweilige Fall als mediationsgeeignet eingestuft wird oder nicht. Dass eine Prüfung der Mediationsgeeignetheit lediglich sekundäre Bedeutung hat, zeigt das Vorgehen am Landgericht Göttingen. Hier ließen sich die Richtermediatoren wahllos Akten geben, sprachen mit den betroffenen Parteien und führten in den meisten Fällen

491 Siehe hierzu die Auswertung bei Rn. 401. 
auch erfolgreich eine Mediation durch. ${ }^{492}$ Trotzdem ist von einem solchen Ansatz einer „Zwangsmediation“ eher abzuraten, da dieser das gesamte Wesen der Mediation, welche sich gerade auf die Freiwilligkeit der einzelnen Parteien, kraft kommunikativer Interaktion den Konflikt beizulegen, ad absurdum führen würde.

Damit bleibt als zweite Alternative eine Anpassung des Deputatsschlüssels. Augenblicklich bekommt der abgebende Fallmanager jeden in der Mediation verhandelten Fall voll auf seinen Deputatsschlüssel angerechnet. Dies ermöglicht ihm zwar auf der einen Seite, Fälle in die Mediation abzugeben und sich auf die rechtlich schwierigen und beweisintensiven Fälle zu konzentrieren, fördert andererseits jedoch geradezu die Abgabe unliebsamer Fälle in die Mediation. ${ }^{493}$ Dem kann nur entgegengewirkt werden, indem die Fälle, welche in der Mediation behandelt werden, dem Fallmanager nicht mehr voll, sondern nur zu einem Teil auf seinen Deputatsschlüssel angerechnet werden. Dieser Ansatz impliziert wiederum das Problem, dass der Fallmanager versucht ist, abzuwägen, ob es für ihn mehr Sinn macht, den Fall selbst zu verhandeln und damit eine volle Anrechnung auf seinen Deputatsschlüssel zu bekommen, oder ob der Fall derart komplex ist, dass er besser stehen würde, müsste er den Fall selbst gar nicht verhandeln und stattdessen lediglich eine anteilige Anrechnung bekommen würde. Je komplexer also ein Fall ist, desto größer wird die Bereitschaft des Fallmanagers zur Abgabe in die Mediation sein. ${ }^{494}$

\section{b) Gefahr eines Kompetenzverlustes}

Die Befürchtung, dass die Abgabe von Fällen in die Mediation dem richterlichen Selbstverständnis, zur Entscheidung von Konflikten mit Mitteln des Rechts berufen zu sein, zuwiderläuft, lässt sich zumindest insoweit nach der statistischen Auswertung entkräften, als dass der überwiegende Teil - nämlich jeweils etwa vier Fünftel - der befragten Richter der Ansicht ist, dass die Rolle des Mediators ohne Entscheidungskompetenzen dem Richteramt keinen Schaden zufügt. Von den insgesamt 94 befragten Richtern gaben 80,8\% ${ }^{495}$ an, dass das Richteramt durch die

\footnotetext{
492 So liegt die Erfolgsquote der Akquisen am LG Göttingen trotz dieses Vorgehens bei 68,75\%, von denen 88,15\% dann sogar in der Mediation erfolgreich sind, siehe Abschlussbericht zur Ausweitung des Begleitforschungsprojekts „Gerichtsnahe Mediation in Niedersachsen“ - PEBB $\ Y$ analoge Datenerhebung zur Gerichtsnahen Mediation.

${ }^{493}$ Vgl. hierzu schon Vorläufiger Abschlussbericht/Zwischenbericht zum Forschungsprojekt „Gerichtsnahe Mediation in Niedersachen, S. 83 f., 85.

494 Siehe hierzu schon Vorläufiger Abschlussbericht/Zwischenbericht zum Forschungsprojekt „Gerichtsnahe Mediation in Niedersachen, S. 87.

$49573,4 \%$ „stimme überhaupt nicht zu“; 7,4\% „stimme eher weniger zu“.
} 
Rolle als Mediator keinen Schaden nehme. Während 7,4\% dieser Aussage weder zustimmen konnten noch sie abzulehnen vermochten, gaben 8,5\%496 der Richter an, dass das Richteramt durch die entscheidungslose Rolle des Mediators Schaden nehme. 3,2\% der insgesamt 94 Befragten machten hierzu keine Angabe.

419 Im Rahmen der Zivilgerichtsbarkeit fällt die Quote derjenigen, die davon ausgehen, dass das Richteramt durch die eher passive Rolle des Mediators keinen Schaden nimmt, etwas geringer aus als bei der Gesamtgruppe, liegt aber immer noch bei 79,55\%.497 Dem stehen 8,86\% ${ }^{498}$ der Richter gegenüber, die einen Schaden für die Entscheidungskompetenz des Richteramtes durch die Mediatorenrolle annehmen. Während sich 3,80\% der im Rahmen der Zivilgerichtsbarkeit insgesamt 79 befragten Richter nicht zu der gestellten Frage äußerten, standen 7,59\% der Frage unentschlossen gegenüber.

420 Bei den sozialgerichtlichen Fällen gaben von den zwölf befragten Richtern 91,67\% $\%^{499}$ an, dass ihrer Ansicht nach die Rolle des Mediators als nicht zur Entscheidung berufenem Richter dem Richteramt keinen Schaden zufügt. Lediglich $8,33 \%$ gaben an, dass sie dieser Aussage vollkommen zustimmen.

421 Insoweit wird durch einen etwaigen Kompetenzverlust der Richterschaft kein negativer Anreiz im Hinblick auf die Mediation gesetzt, sodass dieses Kriterium einer niedersachsenweiten Einführung der gerichtsnahen Mediation nicht im Wege stünde.

\section{Richtermediatoren}

\section{a) Arbeitszeit- und Kostenersparnis}

\section{aa) Zeitersparnis durch Mediation}

422 Dass die Bearbeitung von komplexen, rechtlich komplizierten und/oder durch Beweisschwierigkeiten geprägten Fällen vereinfacht und zeitlich verkürzt werden kann, wurde bereits im Rahmen der PEBB\Y-analogen Datenerhebung festgestellt. Hiernach lässt sich festhalten, dass die Bearbeitungszeit eines Verfahrens vor dem Landgericht im Wege der Mediation rund fünfmal so schnell ist, wie dies im normalen streitigen Verfahren der Fall wäre; in der gesamten erstinstanzlichen

\footnotetext{
496 5,3\% ,stimme vollkommen zu“; 3,2\% ,stimme eher zu“.

$49773,42 \%$,stimme überhaupt nicht zu“; $6,33 \%$ „stimme eher weniger $z u$ “.

498 5,06\% ,stimme vollkommen zu“; 3,80\% ,stimme eher zu“.

$49975,0 \%$,stimme überhaupt nicht zu“; 16,67\% „stimme eher weniger zu“.
} 
Zivilgerichtsbarkeit beträgt die Bearbeitungszeit immerhin noch gut weniger als die Hälfte des streitigen Verfahrens. ${ }^{500}$

Die durch die im Rahmen der PEBB\Y-analogen Datenauswertung gemachte Beobachtung, dass ein Mediationsverfahren in der Regel schneller ist, als dies bei einem normalen streitigen Verfahren der Fall ist, sollte auch im Zusammenhang mit der vorliegenden Datenerhebung noch einmal untersucht werden. Die Aufschlüsselung in eine konkrete Zeitdauer erfolgte in diesem Zusammenhang nicht, da dies den Umfang der Datenerhebung bei weitem gesprengt hätte; vielmehr beschränkte sich die Erhebung darauf, ob die Mediationsverfahren nach Ansicht der Richtermediatoren länger oder kürzer dauerten, als dies in einem streitigen Verfahren der Fall gewesen wäre. Um dennoch eine verwertbare Aussage zu erhalten wurden die Antwortmöglichkeiten in die Gruppen „sehr viel kürzer“, „,viel kürzer“, „etwas kürzer“, „,ungefähr gleich“, „etwas länger“, „viel länger“ eingeteilt.

Von den 274 auswertbaren Fragebögen enthielten insgesamt 257 eine Angabe auf die konkrete Frage, wobei der größte Anteil auch hier wieder durch die landgerichtlichen Fragebögen ${ }^{501}$ gestellt wird. Das Gesamtergebnis stellt sich, wie es nach der Auswertung der PEBB $\ Y$-analogen Daten zu erwarten war, pyramidenförmig dar. Die meisten Richtermediatoren $(39,8 \%)$ gehen davon aus, dass die Mediationsdauer im Vergleich zu einem streitigen gerichtlichen Verfahren „sehr viel kürzer" ist. Immerhin noch 31,4\% der Mediatoren schätzen die Dauer des Mediationsverfahrens als „viel kürzer“ ein. Während 9,9\% der Befragten noch ein einer „etwas kürzeren“ Verfahrensdauer ausgingen, nahmen lediglich 7,7\% an, dass die unterschiedlichen Verfahrensarten „ungefähr gleichlang“ gedauert hätten. Nur jeweils 2,6\% sahen das Mediationsverfahren als „etwas“ bzw. als „viel länger“ an.

Das gleiche oder doch zumindest ein ähnliches Bild ergibt sich bei einer Aufschlüsselung nach den einzelnen Gerichtsbarkeiten für die Zivil-502 und die Sozial-

500 Vgl. hierzu Abschlussbericht zur Ausweitung des Begleitforschungsprojekts „Gerichtsnahe Mediation in Niedersachsen“ - PEBB \Y-analoge Datenerhebung zur Gerichtsnahen Mediation, S. 20 f. Während ein durchschnittliches streitiges Gerichtsverfahren vor einem Landgericht 529,53 Minuten dauert, benötigt man im Durchschnitt für ein landgerichtliches Mediationsverfahren nur 100,92 Minuten. Betrachtet man die gesamten Zivilgerichte der ersten Instanz, so dauert ein streitiges Verfahren im Schnitt immer noch 275,04 Minuten, ein Mediationsverfahren hingegen nur 108,88 Minuten.

501 182, wobei 11 Fragebögen keine Antwort auf diese Frage enthielten.

502 „sehr viel kürzer“ $36,81 \%$, ,viel kürzer“ $33,52 \%$, „,etwas kürzer“ $10,99 \%$, ,ungefähr gleich“ $7,69 \%$, „etwas länger“ $2,75 \%$, ,viel länger“ $2,20 \%$. 
gerichte. ${ }^{503}$ Dem hingegen fallen die Familien- und die verwaltungsgerichtlichen Fragebögen etwas aus dem Rahmen. Während bei den Verwaltungsgerichten zwar niemand von einer längeren Verfahrensdauer ausgeht, ${ }^{504}$ wird bei den Familiengerichten zu einem recht großen Prozentsatz eine viel längere Verfahrensdauer angenommen. ${ }^{505}$ Bei diesem, zumindest bei den Familiengerichten doch sehr stark von dem Gesamtergebnis abweichenden Resultat, stellt sich die Frage, ob dieses aufgrund der geringen Gesamtzahl der auswertbaren Fragebögen,506 zustande gekommen ist oder ob das Mediationsverfahren aufgrund der im Familienrecht doch recht häufig komplexen und festgefahrenen persönlichen Beziehungen länger dauert, als dies der Fall wäre, wenn der gesetzliche Richter den Fall einfach nach den tatsächlichen und rechtlichen Gegebenheiten entscheidet, ohne weiter auf die persönlichen Hintergründe einzugehen. Leider lassen sich hier auch keinerlei Rückschlüsse aus der PEBB\Y-analogen Datenauswertung gewinnen, da auch hier lediglich eine sehr geringe Rückmeldung seitens der Familiengerichte erfolgte.

426 Auch wenn das verwaltungsgerichtliche Ergebnis dem von den Zivilgerichten geprägten Gesamtergebnis recht nahe kommt, kann hier aus statistischen Gründen kein gesicherter Schluss gezogen, sondern allenfalls eine Tendenz festgestellt werden; von den insgesamt 22 von dem Verwaltungsgericht zurückgesandten Fragebögen enthielten immerhin 21 eine Antwort auf die Frage nach der Mediationsdauer im Vergleich zum gerichtlichen Verfahren. Bei den Sozialgerichten kann man hingegen, auch wenn diese mit der Anzahl der zurückgesandten Fragebögen immer noch weit hinter den Zivilgerichten zurückliegen, doch zumindest von einem einigermaßen repräsentativen Ergebnis ausgehen. Von den immerhin 54 Fragebögen enthielten 52 eine Antwort auf die vorliegende Frage.

427 Insoweit lässt sich festhalten, dass das Ergebnis der PEBB\Y-analogen Studie zum Vergleich der Verfahrensdauer in einem streitigen und einem Mediationsverfahren durch die vorliegende Datenerhebung im großen und Ganzen bestätigt wurde, sodass alleine schon aus Zeit- und damit auch aus Kostenersparnisgründen das Mediationsverfahren eine für die Justiz erwägenswerte Alternative darstellt.

\footnotetext{
503 „sehr viel kürzer“ $64,81 \%$, „viel kürzer“ $25,93 \%$, „,etwas kürzer“ $0,0 \%$, ,ungefähr gleich“ $1,85 \%$, „etwas länger“ $3,7 \%$, ,viel länger“ $0,00 \%$.

504 „sehr viel kürzer“ $18,18 \%$, „viel kürzer“ $36,36 \%$, ,etwas kürzer“ $18,18 \%$, „ungefähr gleich“ $22,73 \%$, ,etwas länger“ $0,0 \%$, ,viel länger“ $0,0 \%$.

505 „,sehr viel kürzer“ $18,75 \%$, „viel kürzer“ $18,75 \%$, ,etwas kürzer“ $18,75 \%$, ,ungefähr gleich“ $6,25 \%$, ,etwas länger“" $0,0 \%$, ,viel länger“ $18,75 \%$.

506 16, wovon 3 zu dieser Frage keine Angabe enthielten.
} 


\section{bb) Arbeitsbelastung durch Mediation}

Neben dem reinen zeitlichen Aspekt spielt auch die durch die Mediation entstandene gerichtliche, im Vergleich zu der erfahrungsgemäß in einem solchen Fall ansonsten zu erwartenden Arbeitsbelastung bei der Frage, ob die Bearbeitung von komplexen, rechtlich komplizierten und/oder durch Beweisschwierigkeiten geprägten Fällen vereinfacht werden kann, eine Rolle. Um diesen Aspekt zu untersuchen, wurden ähnliche Kategorien gewählt, wie diejenigen bei der Frage nach der Mediationsdauer im Vergleich zum gerichtlichen Verfahren; namentlich wurde die Aufteilung in „sehr viel geringer“, „viel geringer“, ,etwas geringer“, „, ungefähr gleich“, „etwas höher“, „,viel höher“, „,sehr viel höher" vorgenommen. Nicht nur die gewählten Kategorien sind ähnlich, sondern auch - wie zu erwarten war - die Häufigkeitsverteilung bei der Beantwortung der Frage, so dass sich das Ergebnis auch hier wieder nahezu pyramidenförmig darstellt. Jeweils 32,5\% sahen die Arbeitsbelastung im Mediationsverfahren als „sehr viel geringer“ bzw. als „viel geringer" an, während zumindest noch 16,1\% eine etwas geringere Arbeitsbelastung angenommen haben. Lediglich 6,2\% gingen von einer „ungefähr gleichen“ Belastung aus, $5,8 \%$ von einer „etwas höheren“, $1,1 \%$ von einer viel höheren und nur noch $0,4 \%$ schließlich von einer ,sehr viel höheren“ Arbeitsbelastung; 5,5\% äuBerten sich gar nicht zu dieser Frage. Dieses Gesamtergebnis spiegelt auch die Einzelergebnisse der Zivil-507 und der Sozialgerichtsbarkeit ${ }^{508}$ in etwa wieder, und auch das Verwaltungsgericht weist eine ähnliche Verteilung auf. ${ }^{509}$ Lediglich das Familiengericht fällt, wie schon bei der Frage nach der Dauer des Mediationsverfahrens im Vergleich zum streitigen Verfahren, durch seine abweichende Verteilung auf. ${ }^{510}$ Leider beruhen auch diese Ergebnisse wieder auf nur 16 von dem Familiengericht zurückgesandten Fragebögen, sodass auch hier nicht geklärt werden kann, ob die Abweichung der Wahrscheinlichkeiten auf der geringen Anzahl der der Untersuchung zugrunde liegenden Fälle beruht oder ob sich in diesem Ergebnis familienrechtliche Spezifika widerspiegeln.

\footnotetext{
507 „,sehr viel geringer“ 26,37\%, ,viel geringer“ 33,52\%, ,,etwas geringer“ 19,23\%, ,ungefähr gleich“ $8,24 \%$, ,etwas höher“ 4,95\%, ,viel höher“ $1,65 \%$, ,sehr viel höher“ $0,55 \%$, ,keine Angabe“ $5,49 \%$.

508 ,sehr viel geringer“ $61,11 \%$, ,viel geringer“ $31,48 \%$, ,,etwas geringer“ $5,56 \%$, ,ungefähr gleich“ 0,0\%, ,etwas höher" $0,0 \%$, ,viel höher“ $0,0 \%$, ,sehr viel höher“ $0,0 \%$, ,keine Angabe“ 1,85\%.

509 ,sehr viel geringer“ $27,27 \%$, ,viel geringer“ 40,91\%, ,etwas geringer“ $22,73 \%$, ,, ungefähr gleich“ 4,55\%, ,etwas höher“ $0,0 \%$, „,viel höher“ $0,0 \%$, ,sehr viel höher“ $0,0 \%$, ,keine Angabe“ 4,55\%

510 „,sehr viel geringer“ $12,50 \%$, ,viel geringer“ $12,50 \%$, ,etwas geringer“ $6,25 \%$, ,ungefähr gleich“ 6,25\%, ,etwas höher“ $43,75 \%$, ,viel höher“ $0,0 \%$, ,sehr viel höher“ $0,0 \%$, ,keine Angabe“ $18,75 \%$.
} 
429 In den verbleibenden drei betrachteten Gerichtsbarkeiten ist die Arbeitsbelastung nach Einschätzung der Mediatoren in der Summe wesentlich geringer, als dies bei einem streitigen Gerichtsverfahren der Fall wäre, sodass auch die statistische Untersuchung der Arbeitsbelastung zeigt, dass die Annahme zutrifft, durch die Mediation könne die Bearbeitung von Sachverhalten vereinfacht und zeitlich verkürzt werden.

\section{cc) Erforderlichkeit einer Beweisaufnahme im streitigen Prozess}

430 Ein weiterer statistischer Beleg dafür, dass durch die Mediation die Bearbeitung von komplexen, rechtlich komplizierten und/oder durch Beweisschwierigkeiten geprägten Fällen vereinfacht und zeitlich verkürzt werden kann, ist die Tatsache, dass in den meisten Fällen, wäre es zu einem streitigen Verfahren gekommen, eine Beweisaufnahme erforderlich gewesen wäre. So haben von 274 befragten Richtermediatoren 68,6\% angegeben, dass eine Beweisaufnahme erforderlich gewesen wäre, in 29,6\% hätte es auch im streitigen Verfahren keiner Beweisaufnahme bedurft; $1,8 \%$ haben zu dieser Frage keine Angabe gemacht.

431 Schlüsselt man dieses Gesamtergebnis erneut nach den einzelnen Gerichtsbarkeiten auf, so ergibt sich ein hiervon divergierendes Bild: Bei den Zivilgerichten wird noch wesentlich deutlicher, dass durch die Mediation eine ansonsten erforderliche Beweiserhebung umgangen werden konnte. Hier haben 79,12\% der Befragten angegeben, dass sie im streitigen Verfahren eine Beweiserhebung für erforderlich gehalten hätten, lediglich in 18,68\% hätte es auch hier keiner Beweiserhebung bedurft; 2,2\% machten keine Angaben zu der Frage. In den anderen Gerichtsbarkeiten hält sich die Einschätzung, ob in den einzelnen Fällen eine Beweiserhebung verzichtbar gewesen wäre, in etwa die Waage. Bei dem Sozialgericht wäre zu $50,0 \%$ eine Beweiserhebung erforderlich gewesen, in 48,15\% hätte es einer solchen nicht bedurft (1,85\% enthielten sich einer Angabe). Bei den Familien- und Verwaltungsgerichtssachen verhält es sich ganz ähnlich: Hier hielten $43,75 \%$ beim Familiengericht und $45,45 \%$ beim Verwaltungsgericht eine Beweisaufnahme im streitigen Verfahren für erforderlich. Da hier jeder der 16 bzw. 22 zurückgesandten Bögen eine Antwort auf die gestellte Frage enthielt, hielten im Gegenzug bei den Familiengerichtssachen 56,25\% bzw. 54,55\% beim Verwaltungsgericht eine Beweiserhebung auch im streitigen Prozess für entbehrlich.

432 Somit lässt sich festhalten, dass in der Zivilgerichtsbarkeit zu 4/5, in den anderen Gerichtsbarkeiten immer noch in der Hälfte der Fälle, auf eine Beweisaufnahme verzichtet werden kann. Dies führt nicht nur dazu, dass die einzelnen Verfahren schneller behandelt werden bzw. mehr Zeit für andere Probleme bleibt, sondern auch, dass zu einem guten Teil die mit dem Einbringen von Beweismitteln, wie zum Beispiel Personenzeugen, einhergehenden Unsicherheiten vermieden werden 
können. Die Gefahr, dass sich das im Verfahren gefundene Ergebnis auf falsche Beweise gründet, ist somit geringer.

\section{dd) Kostenersparnis in Bezug auf eine etwaige Beweiserhebung}

Mit der Frage nach dem Einsparen eine Beweiserhebung geht auch die Frage der Kostenersparnis einher, um deren Beantwortung die Richtermediatoren im Zusammenhang mit der Erforderlichkeit einer Beweiserhebung in einem streitigen Verfahren gebeten wurden. Von den insgesamt 274 zurückgesandten Fragebögen enthielten leider 112 keine Angabe über die geschätzten Kosten einer möglichen Beweiserhebung; das entspricht einer Quote von 40,9\%. 20,5\%, schätzten dass durch die ersparte Beweiserhebung bis zu $999 €$ gespart werden konnten. Immerhin noch 13,3\% sahen die Ersparnis zwischen 1.000 bis zu $1.999 €$ und noch einmal fast ebenso viele, nämlich 12,5\% nahmen an, dass durch die Mediation und dem damit einhergehenden möglichen Verzicht auf eine Beweisaufnahme 2.000 bis $2.999 €$ gespart werden konnten. Nur noch knapp 5,8\% sahen die Einsparung in der Größenordnung von 3.000 bis $3.999 €$. Die übrigen Angaben in der Gesamtsumme von 7,3\% machten Angaben, die bis zu einer Einsparung von maximal $15.000 €$ reichten. Die Einzelangaben bei den entsprechenden Beträgen sind jedoch so gering ausgefallen, dass es sich hier offenbar nur um einzelne Extremfälle handelt, die nicht typisch für die gemachten Einsparungen sind. Nichtsdestotrotz belegt diese Statistik, dass die Gerichtsnahe Mediation geeignet ist, neben Zeitersparnissen und Arbeitserleichterungen auch Kosten, welche in einem gewöhnlichen streitigen Verfahren entstehen, einzusparen.

\section{ee) Zeitersparnis durch Mediation}

Bei der ökonomischen Analyse des Rechts wurde ferner die Annahme aufgestellt, dass die Fälle, die in die Mediation abgegeben werden, durch die Prägung und Bestimmung des Verhandlungsablaufs der Mediation durch die Parteien einer kürzeren Bearbeitungszeit unterliegen. Ob dies an der Beeinflussung des Verhandlungsablaufs der Mediation durch die Parteien erfolgte, lässt sich aufgrund der statistischen Auswertung nicht erkennen; erkennbar ist jedoch, dass die Richter zum überwiegenden Teil den Eindruck haben, dass die Verfahrensdauer eines Mediationsverfahrens im Vergleich zum Gang des streitigen Verfahrens kürzer ist. So gaben bei der Gesamtauswertung von 94 befragten Richtern 45,7\% an, dass das Mediationsverfahren schneller war, als dies bei einem Gang im streitigen Verfahren der Fall gewesen wäre. Für 23,4\% war kein Unterschied im Hinblick auf die Verfahrensdauer bei einem Mediations- und bei einem streitigen Verfahren zu erkennen, während 18,1\% der Richter angaben, dass die Mediation länger gedauert hätte als ein gewöhnliches Gerichtsverfahren. Die übrigen 12,8\% der befragten Richter äußerten sich nicht zu dieser Frage. 
435 Im Bereich der Zivilgerichte gaben 43,04\% - und damit der überwiegende Prozentsatz - der 79 befragten Richter an, eine Zeitersparnis im Vergleich zum gewöhnlichen streitigen Verfahren empfunden zu haben. Während bei 20,25\% das Mediationsverfahren sich als zeitintensiver erwies als das gewöhnliche Verfahren, gehen $24,05 \%$ der Richter von einer etwa gleichen Verfahrensdauer aus. Die übrigen 12,66\% machten keine Angabe zu dieser Frage.

436 Bei den sozialgerichtlichen Streitigkeiten gaben von den zwölf befragten Richtern zwei Drittel $(66,67 \%)$ an, dass die Mediation im Vergleich zu gewöhnlichen Prozessen das schnellere Verfahren sei, während $16,67 \%$ von einer gleichen Dauer ausgingen. Während kein Richter angab, das Mediationsverfahren hätte länger gedauert als die Mediation, äußerten sich 16,67\% nicht zu der obigen Frage.

437 Insoweit werden auch in diesem Zusammenhang die Ergebnisse der PEBB\Yanalogen Datenauswertung bestätigt.

438 Weniger eindeutig gestaltet sich das statistische Ergebnis bei der Frage, inwieweit eine Mediation sich im Vergleich zu einem richterlichen Vergleich als die zeitsparendere Alternative darstellt. Während 30,9\% der befragten Richter hier keinen Unterschied zwischen der Dauer eines richterlichen Vergleichs und eines Mediationsverfahrens sahen, gaben 29,8\% der befragte Richter an, dass die Mediation der zeitsparendere Weg sei, während 26,6\% angaben, der richterliche Vergleich sei die weniger zeitintensive Variante. 12,8\% der 94 befragten Richter äußerten sich nicht zum diesem Komplex. Insoweit ergibt sich aus der Gesamtauswertung, dass sich die Verfahrensdauer von richterlichen Vergleichen und von gerichtsnahen Mediationsverfahren in der Gesamtbetrachtung in etwa die Waage hält.

439 Nichts anderes gilt für die Einzelbetrachtung der Zivilgerichte. Auch hier ist das Verhältnis zwischen denjenigen Richtern, die angaben, dass der richterliche Vergleich weniger zeitaufwendig war $(29,11 \%)$ als die Mediation zu denen, die die Mediation als zeitsparendere Alternative ansehen (26,58\%), recht ausgeglichen. Keine Unterschiede ergaben sich für 31,65\% der befragten Richter. Von den 79 Befragten machten 12,66\% der Richter der Zivilgerichtsbarkeit keine Angabe zu der gestellten Frage.

440 Bei den Sozialgerichten hingegen gab mit 50,0\% der befragten Richter der überwiegende Teil an, dass das gerichtsnahe Mediationsverfahren auch im Verhältnis zum richterlichen Vergleich die zeitsparendere Alternative darstellt, wohingegen lediglich 8,33\% der Ansicht sind, dass der richterliche Vergleich vom Zeitaufwand her geringer ist. Ein Viertel der Befragten gab an, keine Unterschiede bei der Dauer festgestellt zu haben. Auch wenn dieses Ergebnis im Rahmen der Sozialgerichtsbarkeit äußerst positiv für die Mediation ausfällt, so muss hier doch berücksichtigt werden, dass sich die angegebenen Quoten auf eine recht geringe Gesamt- 
anzahl an Fragebögen, nämlich auf nur zwölf, stützt, wobei darüber hinaus 16,67\% sich nicht zu der Frage nach der kürzeren Verfahrensdauer bei richterlichen Vergleichen und gerichtsnaher Mediation äußerten.

Insoweit lässt sich zwar auch im Verhältnis zwischen dem richterlichen Vergleich und dem Mediationsverfahren feststellen, dass zwar im Hinblick auf die Mediation keine negativen Anreize gesetzt werden, die positiven Anreize jedoch eher marginal sind.

\section{ff) Arbeitsbelastung}

Als positiver Anreiz für die Richterschaft im Hinblick auf die gerichtsnahe Mediation wurde im Rahmen der ökonomischen Analyse des Rechts angenommen, dass die Tätigkeit als Mediator im Vergleich zu der eines Richters im gewöhnlichen streitigen Prozess weniger belastend sei. Zum einen kann sich diese geringere Belastung im Hinblick auf eine gesamtgerichtliche Arbeitsbelastung und darüber hinaus auch auf eine persönliche Arbeitsbelastung für den Richter auswirken. Im Rahmen der empirischen Begleitforschung wurden diese beiden Aspekte sowohl für den Vergleich des streitigen Gerichtsverfahrens mit der Mediation als auch des gerichtlichen Vergleichs mit der Mediation untersucht.

Betrachtet man zunächst die gesamtrichterliche Arbeitsbelastung bei der gerichtsnahen Mediation im Hinblick auf das streitige Verfahren, so ergibt sich, dass lediglich ein geringer Teil der befragten Richter, nämlich 11,7\%, davon ausgehen, dass die Arbeitsbelastung im Zusammenhang mit der gerichtsnahen Mediation höher ist, als dies bei einem gewöhnlichen streitigen Verfahren der Fall wäre. Dem stehen $37,2 \%$ der Richter gegenüber, die angeben, dass die gesamtgerichtliche Arbeitsbelastung durch die Mediation geringer ausfällt, als dies im Wege des streitigen Verfahrens der Fall wäre. Für 38,3\% stellt sich die Belastung als ungefähr gleich dar. Von den insgesamt 94 befragten Richtern machten 12,8\% keine Angabe zu der Frage nach der gesamtgerichtlichen Arbeitsbelastung. Insoweit lässt sich im Zusammenhang mit der gesamtgerichtlichen Arbeitsbelastung erkennen, dass durch sie tatsächlich positive Anreize für die Richterschaft im Hinblick auf eine gerichtsnahe Mediation geschaffen werden, da die Zahl derer, die die Arbeitsbelastung als geringer ansehen die derer, die eine Mediation mit einem Mehraufwand verbinden, deutlich übersteigt.

Auch bei einer Aufschlüsselung in die Gerichtsbarkeiten, die in diesem Zusammenhang einzeln untersucht werden konnten, ergibt sich ein ähnliches Bild. Bei den Zivilgerichten gaben von 79 befragten Richtern 37,97\% an, dass der gesamtgerichtliche Arbeitsaufwand durch die gerichtsnahe Mediation geringer liegt, als dies im streitigen Verfahren der Fall ist. Der gleiche Prozentsatz, also ebenfalls 
$37,97 \%$ der Befragten gab an, dass die Mediation zumindest nicht mit einem Mehraufwand verbunden ist, sondern sich die Arbeitsbelastung in etwa die Waage hält. Lediglich 11,39\% schätzen den gesamtgerichtlichen Arbeitsaufwand durch ein Mediationsverfahren höher ein als bei einem streitigen Verfahren. Die übrigen 12,66\% äußerten sich nicht zu der gestellten Frage.

445 Noch deutlicher fallen die Quoten im Bereich der Sozialgerichtsbarkeit aus. Hier ist die Mediation für lediglich 8,33\% der Richter mit einer gesamtgerichtlich höheren Arbeitsbelastung verbunden als die streitigen Verfahren. Während es für ein Drittel, also 33,33\% der Befragten keinen Unterschied macht, ob ein Verfahren im Rahmen eines streitigen Verfahrens oder im Wege der Mediation durchgeführt wird, gaben 41,67\% an, dass die Arbeitsbelastung für den gesamten Gerichtsapparat durch die Mediation geringer ausfällt. Bei der Auswertung dieser Zahlen für die Sozialgerichte gilt es jedoch zu berücksichtigen, dass die gefundenen Ergebnisse lediglich als Tendenzen zu sehen sind, da die Gesamtzahl der der Untersuchung zugrunde liegenden Fragebögen mit zwölf relativ gering ist. Zudem kommt noch hinzu, dass sich 16,67\% - absolut zwei Richter - nicht zu der gestellten Frage geäußert haben.

446 Auf eine Einzeldarstellung der Verwaltungsgerichte soll in diesem Zusammenhang verzichtet werden, da hier lediglich ein Fragebogen für die Auswertung zur Verfügung stand, so dass eine sinnvolle Aussage nicht zu treffen ist.

447 Während schon die gesamtgerichtliche Arbeitsbelastung im Zusammenhang mit der Mediation im Vergleich zum normalen streitigen Verfahren zu großen Teilen als geringer angesehen wurde, so wird dies für die persönliche Arbeitsbelastung des mit einer Mediation betrauten Richters noch deutlicher. Hieran zeigt sich, dass sich für einen Richter, der als Mediator tätig ist, wie es schon im Rahmen der ökonomischen Analyse angenommen wurde, durchaus positive Anreize im Hinblick auf eine Mediatorentätigkeit ergeben. In Zahlen stellt sich der Sachverhalt wie folgt dar:

448 Nach der Gesamtauswertung war es für 42,6\% der insgesamt 94 befragten Richter so, dass die persönliche Arbeitsbelastung, wie sie im Bereich der Mediaiton anfällt, sich als geringer darstellte, als dies im streitigen Gerichtsverfahren der Fall ist. Für weitere 34,0\% ergaben sich bezüglich der Arbeitsbelastungen keine Unterschiede zwischen den beiden Verfahrensarten. Lediglich 11,7\% der befragten Richter gaben an, dass die Arbeitsbelastung im Mediationsverfahren höher als in einem vergleichbaren streitigen Verfahren wäre. Die restlichen 11,7\% der Richterschaft äußerten sich nicht zu dieser Frage.

449 Die Einzelstatistik für die Zivilgerichtsbarkeit weicht nur leicht von der Gesamtbetrachtung ab, was sicherlich zu einem Großteil dadurch bedingt ist, dass 79 
Fragebögen, der der Gesamtauswertung insgesamt zugrunde liegenden 94 Fragebögen aus diesem Bereich stammen. Hier gaben $41,77 \%$ an, dass sie durch die Mediation einer geringeren Arbeitsbelastung ausgesetzt sind, als dies im streitigen Verfahren der Fall ist. Dass dieses weniger belastend sei, gaben nur 12,66\% an. Für 34,18\% stellt sich die Arbeitsbelastung ungefähr gleich dar. Die übrigen 11,39\% machten hierzu keine Angabe.

Noch günstiger im Hinblick auf etwaige positive Anreize im Zusammenhang mit der Mediation stellt sich die Lage beim Sozialgericht dar, auch wenn es hier zur berücksichtigen gilt, dass sich die Auswertung lediglich auf zwölf Fragebögen stützt, von denen lediglich 16,67\% eine Angabe zu der gestellten Frage enthalten. Dennoch gaben hier 58,33\% der Richter an, dass die Mediation für sie persönlich eine geringere Arbeitsbelastung darstelle, als dies im streitigen Verfahren der Fall sei. 8,33\% hingegen sahen die Mediation im Vergleich zum streitigen Verfahren als belastender an, während 16,67\% keine Unterschiede im Hinblick auf die Arbeitsbelastung feststellen konnten.

Insofern ergeben sich bei einem Vergleich des gewöhnlichen streitigen Verfahrens mit dem der Mediation, wie schon in der ökonomischen Analyse gemutmaßt positive Anreize für einen Richter, Verfahren in der Mediation zu behandeln, weil dies zum einen die gesamtgerichtliche Arbeitsbelastung senkt, als auch zum anderen die persönliche Arbeitsbelastung des mit der Mediation betrauten Richters verringert.

Im Bereich der gesamtgerichtlichen Arbeitsbelastung bezüglich gerichtlicher Vergleiche gegenüber der Mediation ergeben sich für die Richterschaft weniger positive Anreize. So gaben hier von den 94 Befragten lediglich 16,0\% an, dass die Mediation auf gesamtgerichtlicher Ebene eine geringere Arbeitsbelastung darstellen würde. Dem stehen $25,5 \%$ der Richterschaft gegenüber, die die Mediation vielmehr als arbeitsintensiver ansehen. Der Großteil der Befragten (45,7\%) sieht keine Unterschiede bezüglich der Arbeitsbelastung. Die übrigen 12,8\% äußerten sich nicht zu der Frage.

Im Bereich der Zivilgerichtsbarkeit gestaltet sich die Situation hinsichtlich der Mediation als marginal positiver. Hier gaben immerhin 17,72\% der Richter an, dass die gesamtgerichtliche Arbeitsbelastung durch Mediation im Vergleich zu den gerichtlichen Vergleichen geringer ausfällt. Während 45,57\% der Richterschaft hier keine Unterschiede feststellen konnten, war die mit der Mediation verbundene Arbeitsbelastung für 24,05\% der Befragten größer. Die übrigen 12,66\% der 79 befragten Zivilrichter äußerten sich nicht zu dieser Frage.

Noch deutlicher als die Gesamtauswertung gestaltet sich die Einzelauswertung der Sozialgerichtsbarkeit, auch wenn es hier zu berücksichtigen gilt, dass der Auswer- 
tung lediglich zwölf Fragebögen zugrunde liegen, von denen zusätzlich auch noch 16,67\% keine Angabe zu der Frage enthalten, sodass man dieses Ergebnis lediglich als Tendenz und nicht als gesicherte statistische Erkenntnis werten darf. Hier waren es 8,33\% der Richter, die die gesamtgerichtliche Arbeitsbelastung durch ein Mediationsverfahren geringer ansahen als dies bei einem gerichtlichen Vergleich der Fall gewesen wäre. Den umgekehrten Fall hingegen nehmen 33,33\% der Befragten an. Für 41,67\% macht es keinen Unterschied in Bezug auf die gesamtgerichtliche Arbeitsbelastung, ob ein richterlicher Vergleich oder eine gerichtsnahe Mediation durchgeführt wird.

455 Da sich durch die Mediation im Hinblick auf den richterlichen Vergleich der status quo nicht sonderlich ändern würde, könnte man unter diesem Gesichtspunkt auf die Einführung einer niedersachsenweiten Mediation verzichten, da sich insoweit mehr negative als positive Anreize ergeben. Wie sich im Folgenden jedoch zeigen wird, stellt sich die Lage für die persönliche Arbeitsbelastung des einzelnen Richters wiederum anders dar. Auch, wenn die gesamtgerichtliche Arbeitsbelastung von den Richtern bei einem Mediationsverfahren als höher empfunden wird, als dies bei einem richterlichen Vergleich der Fall ist, so ergibt die empirische Auswertung doch erstaunlicherweise, dass die Richter ihre persönliche Arbeitsbelastung im Zusammenhang mit der Mediation im Durchschnitt als niedriger ansehen, sodass sich für die Richterschaft auch hier die im Rahmen der ökonomischen Analyse getroffene Vermutung, dass durch die Mediation für die Richter positive Anreize durch geringere Belastungen geschaffen werden, bestätigt. Von den insgesamt 94 befragten Richtern gaben nämlich 29,8\% an, dass ihre persönliche Arbeitsbelastung bei einem Mediationsverfahren geringer ausfällt, als dies bei einem richterlichen Vergleich der Fall wäre. Den umgekehrten Fall, dass die persönliche Arbeitsbelastung bei einem richterlichen Vergleich geringer ist, nahmen hingegen nur $23,4 \%$ an. Für 35,1\% macht es für die persönliche Arbeitsbelastung keinen Unterschied, ob ein Verfahren durch eine Mediation oder durch einen richterlichen Vergleich geregelt wird. Die übrigen 11,7\% machten zu dieser Frage keine Angaben.

456 Im Bereich der Zivilgerichtsbarkeit gestalten sich die Verhältnisse zwischen den Richtern, die eine geringere persönliche Arbeitsbelastung im Bereich der Mediation annehmen, und denen, welche eine solche bei einem richterlichen Vergleich spüren, als recht ausgeglichen. Hier gehen $27,85 \%$ der 79 befragten Richter von einer geringeren Belastung im Bereich der Mediation aus; dem stehen 25,32\% der Richterschaft gegenüber, für die sich die Arbeitsbelastung bei einem richterlichen Vergleich als geringer darstellt. Während 35,44\% hier keine Unterschiede sehen, machten 11,39\% keine Angaben hierüber. 
Im Bereich der Sozialgerichtsbarkeit hingegen zeigen sich Tendenzen - eine genaue Aussage ist aufgrund des geringen Zahlenmaterials nicht möglich -, dass die Mediation auch im Hinblick auf einen richterlichen Vergleich die für den Richter weniger belastende Alternative ist. Hier gaben 50,0\% der zwölf befragten Richter an, dass dem so sei. Während sich für ein Viertel der Richterschaft hier keine Unterschiede zwischen einem richterlichen Vergleich und einer Mediation zeigen, war die persönliche Arbeitsbelastung für 8,33\% der Richter bei einer Mediation höher. 16,67\% äußerten sich nicht zu dieser Frage.

Insofern lohnt sich die Einführung der gerichtsnahen Mediation im Verhältnis zu einem richterlichen Vergleich schon aus dem Grund, dass die persönliche Arbeitsbelastung der einzelnen Richter mit einer größeren Wahrscheinlichkeit geringer ausfällt. Insoweit könnte die Zufriedenheit der Richter mit ihrem Berufsverhältnis durch die Einführung der gerichtsnahen Mediation gesteigert werden.

\section{gg) Ausbildungskosten}

Im Rahmen der ökonomischen Analyse des Rechts wurde die These aufgestellt, dass momentan zwar Kosten für die Zusatzausbildung der Mediatoren entstehen, was einen negativen Anreiz darstellt, dass diese Kosten aber dann wieder entfallen, wenn der Erwerb der für die Mediation erforderlichen Fähigkeiten im Ausbildungskanon etabliert wird.

Damit einher geht zunächst die Frage, ob man die Fähigkeit zur mediativen Konfliktlösung überhaupt erlernen kann. Die zu diesem Bereich befragten Richter gaben zu einem überwiegenden Teil, nämlich zu 47,8\% $0^{511}$ an, dass man die Fähigkeiten eines Mediators durchaus erlernen kann. Während 23,4\% hier indifferent waren, gaben $20,2 \% 512$ an, dass die Fähigkeit zu mediieren ,angeboren“ sein muss und man sie dementsprechend nicht erlernen könnte. Die übrigen 8,5\% der insgesamt 94 befragten Richter machten keine Angabe zu der Frage nach der Erlernbarkeit der Mediatorenfähigkeiten.

Splittet man dieses Gesamtergebnis nach den Gerichtsbarkeiten auf - wobei hier die Zivil- und Familiengerichte zusammengefasst sind, um aussagekräftigere Ergebnisse zu erzielen und das Verwaltungsgericht aufgrund einer zu geringen Anzahl an Fragebögen (es liegt nur einer vor) aus der Einzelbetrachtung ausgenommen wird - so ergibt sich für die Zivilgerichtsbarkeit lediglich ein leicht abgewan-

\footnotetext{
$51125,5 \%$,stimme überhaupt nicht zu“; 22,3\% „stimme eher weniger zu“.

512 6,4\% ,stimme vollkommen zu“; $13,8 \%$,stimme eher zu“.
} 
deltes Bild. Hier gaben 49,37\% $0^{513}$ der befragten Richter an, dass man die Mediatorenfähigkeiten durchaus erlernen könne, während hingegen 20,25\%514 gegenteiliger Ansicht waren. 21,52\% waren sich unschlüssig, während 8,86\% der insgesamt 79 befragten Richter keine Angabe zu dieser Frage machten.

462 Bei der Sozialgerichtsbarkeit gaben genau 50,0\%515 der Richter an, dass sie der Ansicht seien, dass die Mediatorentätigkeit durchaus erlernbar sei; $25,0 \%$ hingegen 516 sehen die Fähigkeiten eines Mediators für nicht erlernbar an. Hier waren es 16,67\%, die der Frage indifferent gegenüber standen, während 8,33\% der insgesamt zwölf Sozialrichter sich nicht zu der Frage äußerten. Aufgrund der geringen Gesamtzahl der befragten Sozialrichter kann dieses Ergebnis jedoch auch nur wieder als Hinweis, nicht hingegen als statistische Erkenntnis gewertet werden.

463 Fraglich in diesem Zusammenhang ist jedoch weiter, ob es überhaupt erforderlich ist, die Fähigkeiten eines Mediators zu vermitteln oder ob dies nicht vielmehr dadurch überflüssig wird, dass der Richter durch seine Berufsausübung schon genügend Erfahrungen gesammelt hat, sodass eine solche Ausbildung sich als überflüssig darstellen würde. Danach befragt, gab der größte Teil der Richterschaft, nämlich $65,9 \% 517$ an, dass die Erfahrungen im Richteramt eine spezielle Mediatorenausbildung nicht zu ersetzen vermögen; dieser Auffassung waren lediglich 10,6\%.518 14,9\% waren indifferent im Hinblick auf diese Frage, während 8,5\% der insgesamt 94 Befragten keine Angabe zu dieser Fragestellung machten.

464 Eine Aufschlüsselung in die einzelnen Gerichtszweige ergibt eine vergleichbare Wahrscheinlichkeitsverteilung. Bei den Zivilgerichten gaben 67,09\% ${ }^{519}$ an, dass eine spezielle Ausbildung zum Mediator nicht durch richterliche Erfahrung zu substituieren ist; lediglich 11,39\% $0^{520}$ der Zivilrichter gehen von einer solchen Substituierbarkeit aus. 12,66\% waren indifferent bezüglich dieser Frage, während 8,86\% der 79 befragten Richter keine Angabe zu dieser Frage machten.

$51324,05 \%$,stimme überhaupt nicht zu“; 25,32 „stimme eher weniger zu“.

$5147,59 \%$,stimme vollkommen zu“; $12,66 \%$,stimme eher zu“.

$51541,67 \%$,stimme überhaupt nicht zu“; $8,33 \%$,stimme eher zu“.

$5160,0 \%$,stimme vollkommen zu“; $25,0 \%$,stimme eher zu““.

$51746,8 \%$,stimme überhaupt nicht zu“; $19,1 \%$,stimme eher weniger zu“.

518 2,1\% ,stimme vollkommen zu“; 8,5\% „stimme eher zu“.

$51945,57 \%$,stimme überhaupt nicht zu“; $21,52 \%$,stimme eher nicht zu“

520 2,53\% „stimme vollkommen zu“; $8,86 \%$,stimme eher zu“. 
Ähnlich auch die Wahrscheinlichkeitsverteilung bei der Sozialgerichtsbarkeit. Hier gehen 66,66\% $0^{521}$ davon aus, dass eine mediative Ausbildung nicht durch die Erfahrungen, die man als Richter gesammelt hat, zu kompensieren ist. Dieser Ansicht waren lediglich 8,33\%.522 Während 16,67\% indifferent in Bezug auf diese Frage waren, machten 8,33\% der insgesamt zwölf befragten Richter keine Angabe zu dieser Frage.

Dementsprechend ist eine Ausbildung zum Mediator unabdingbar, auch wenn derjenige, der als Mediator fungieren möchte, schon jahrelange Erfahrungen als Richter hat. Dass die entsprechenden Kompetenzen jedoch nicht bereits im Studium der Rechtswissenschaften selbst vermittelt werden, sondern in den Ausbildungskanon erst integriert werden müssten, wird deutlich, wenn man sich die Befragung der Richter bezüglich des Unterweisens in den nötigen Fähigkeiten zur Mediation im Rahmen des Jurastudiums betrachtet.

Von den insgesamt 94 befragten Richtern gaben $82,9 \% 0^{523}$ an, dass die zu der Durchführung einer Mediation erforderlichen Kompetenzen nicht bereits im Studium vermittelt werden; hiervon gingen lediglich $3,2 \% 524$ aus. 3,2\% standen dieser Frage unentschieden gegenüber, während 10,6\% hierzu keine Angabe machten.

Unterteilt in die einzelnen Gerichtsbarkeiten ergibt sich folgende Wahrscheinlichkeitsverteilung: Bei den Zivilrichtern sind 82,28\%525 der Ansicht, dass keine Grundlagen für die Mediatorentätigkeit im Studium gelegt werden; hiervon gehen nur 3,8\% 526 aus. Während $11,39 \%$ der insgesamt 79 befragten Richter keine Angabe zu der Frage machten, standen ihr 2,53\% der Richter indifferent gegenüber.

Die Sozialrichter gaben zu 83,34\% $0^{527}$ an, die Grundlagen für die Durchführung einer Mediation nicht im Jurastudium vermittelt bekommen zu haben; hiervon ging im Bereich der Sozialgerichtsbarkeit niemand aus. Lediglich 8,33\% der zwölf Sozialrichter war unentschieden bezüglich dieser Frage und der gleiche Prozentsatz, also ebenfalls 8,33\% machten keine Angabe zu dieser Frage.

Da eine Ausbildung zum Mediator, wie die empirische Untersuchung belegt hat, für die erfolgreiche Durchführung einer Mediation unbedingt erforderlich ist, die

\footnotetext{
$52158,33 \%$,stimme überhaupt nicht zu“; $8,33 \%$,stimme eher weniger zu“.

$5220,0 \%$,stimme vollkommen zu“; $8,33 \%$ „stimme eher zu“.

$52357,4 \%$,stimme überhaupt nicht zu“; $25,5 \%$ „stimme eher weniger zu“.

$5243,2 \%$,stimme vollkommen zu“; $0,0 \%$,stimme eher weniger zu“.

$52554,43 \%$,stimme überhaupt nicht zu“; $27,85 \%$ „stimme eher weniger zu“.

526 3,8\% „stimme vollkommen zu“; 0,0\% „stimme eher zu“.

$52766,67 \%$,stimme überhaupt nicht zu“; $16,67 \%$ „stimme eher weniger zu“.
} 
Grundlagen jedoch (noch) nicht während des Studiums gelegt werden, sollte genau hier angesetzt und die Juristenausbildung dahingehend geändert werden, dass den Studenten von Anfang an die Fähigkeit zur mediativen Konfliktbeilegung an die Hand gegeben werden. Damit entfallen auf die Dauer auch die Kosten für etwaige Fortbildungsveranstaltungen für fertig ausgebildete Juristen.

\section{b) Zufriedenheit mit der beruflichen Tätigkeit}

471 Für die Mediation kann auch durch die Zufriedenheit und Motivation der Richter dadurch gesteigert werden, dass er im Rahmen der Mediation eine Kooperation zwischen den Parteien fördern kann und nicht dazu gezwungen ist, ihren Streit zu entscheiden.

472 Die statistische Untersuchung zur Belastung durch Mediation im Vergleich zur sonstigen Arbeitsbelastung in einem streitigen Verfahren kann nicht nur, wie bereits geschehen, für die Untersuchung, ob die Mediation eine Vereinfachung und Beschleunigung des jeweiligen Prozesse bewirkt, herangezogen werden, ${ }^{528}$ sondern mit ihrer Hilfe kann auch nachgewiesen werden, dass die Mediationsverfahren für den Richtermediator nicht so belastend sind, wie das bei einem normalen Verfahren der Fall wäre.

473 Nach den Angaben des Sozial- und des Verwaltungsgerichts fiel die Arbeitsbelastung generell geringer aus, als dies in einem streitigen Verfahren zu erwarten gewesen wäre. Bei den Zivilgerichten verhält es sich zum überwiegenden Teil ähnlich. Einzige Ausnahme stellen hier wieder die Familiengerichte dar, wo sich geringere und höhere Belastungen in einem Vergleich zum streitigen Verfahren die Waage halten. Trotzdem soll auch an dieser Stelle noch einmal darauf hingewiesen werden, dass in dem Ergebnis des Familiengerichts aufgrund der geringen Zahl der auswertbaren Fragebögen kein statistisch gesichertes Ergebnis, sondern allenfalls eine vorsichtige Tendenz gesehen werden kann.

\section{c) Beförderungschancen und Verbesserung der Berufschancen}

474 Ein weiterer positiver Anreiz im Zusammenhang mit der Mediation könnte die Verbesserung von Berufs- und Beförderungschancen sein. In diesem Zusammenhang wurden die Richterinnen und Richter befragt, ob sie der Ansicht sind, dass die Mediation ihre Berufschancen zum einen im Rahmen der Justiz und zum anderen im Bereich der Privatwirtschaft steigert.

\footnotetext{
528 Siehe hierzu Rn. 428.
} 
Im Hinblick auf die Berufs- und Beförderungschancensteigerung im Bereich der Justiz durch die Mediation lässt sich die aufgestellte These nach der statistischen Auswertung so nicht halten. Von den insgesamt 94 befragten Richtern gaben $53,2 \%$ an, dass ihre Berufsaussichten in der Justiz durch ihre Tätigkeit als Mediator nicht steigen würden; hiervon waren lediglich 35,1\% der Befragten überzeugt, die übrigen 11,7\% machten hierzu keine Angabe.

Ähnlich gestaltet sich die Wahrscheinlichkeitsverteilung im Bereich der Zivilgerichtsbarkeit. Hier gaben 53,16\% der befragten Richter an, keine besseren Berufschancen durch ihre Mediatorentätigkeit zu haben, während 34,18\% der gegenteiligen Überzeugung waren. 12,66\% der insgesamt 79 in diesem Bereich befragten Richter äußerten sich nicht zu der Frage.

Die Wahrscheinlichkeitsverteilung bei den Sozialgerichten hingegen stellt sich recht ausgeglichen dar. Von den zwölf befragten Richtern gab jeweils die Hälfte an, dass ihre Tätigkeit als Mediator ihre beruflichen Aussichten in der Justiz steigern würden bzw. dass dem nicht so sei.

Anders hingegen stellt sich die Lage bei der Frage dar, ob die Tätigkeit als richterlicher Mediator die Berufschancen des betroffenen Richters auf dem privaten Arbeitsmarkt steigern würde. Hier gehen von den 79 insgesamt befragten Richtern $50,0 \%$ von einer solchen Steigerung ihrer Berufschancen aus. Lediglich 31,9\% glauben nicht, dass mit der Mediatorentätigkeit eine Steigerung ihrer Berufschancen außerhalb der Justiz einhergeht. Die übrigen 18,1\% äußerten sich nicht zu dieser Frage.

Nicht ganz so positiv wie im Rahmen der Gesamtauswertung stellt sich die Wahrscheinlichkeitsverteilung dar, wenn man den Bereich der Zivilgerichtsbarkeit für sich genommen betrachtet. Hier sind es 45,57\% der Zivilrichter, die an eine Steigerung ihrer Berufschancen auf dem privaten Arbeitsmarkt glauben, weil sie als Richtermediator tätig waren oder sind. Dem stehen jedoch 35,44\% der Richter gegenüber, die nicht von einer solchen Steigerung ausgehen. 18,99\% der 79 befragten Richter enthielten sich einer Angabe bezüglich dieser Frage.

Bei der Sozialgerichtsbarkeit hingegen sind es 83,33\% die eine Chancensteigerung annehmen, während lediglich 16,67\% ein solches nicht annehmen. Alle zwölf befragten Richter äußerten sich zu dieser Frage.

Insofern lässt sich feststellen, dass im Bereich der Verbesserung der Berufschancen die Richter in ihrer subjektiven Wahrnehmung durchaus positive Anreize sehen, allerdings eher im Bereich der Privatwirtschaft und nicht in der Justiz. Nichtsdestotrotz kann dies die Richterschaft dazu bewegen, der Mediation aufgeschlossen gegenüber zustehen. 


\section{Anreizstrukturen Anwälte}

\section{Gebührenordnungen}

482 Durch die statistische Erhebung im Hinblick auf einen möglichen Einnahmeverlust der Rechtsanwälte, wenn sie ein Verfahren in der Mediation und nicht im Wege des gewöhnlichen streitigen Verfahrens betreiben, bestätigt sich die Annahme, die im Rahmen der ökonomischen Analyse aufgestellt wurde: Für die Rechtsanwälte, sollten sie ihrem Mandaten zu einem gerichtsnahen Mediationsverfahren raten, ergeben sich zumindest finanziell keine negativen Anreize.

483 Von den 304 befragten Anwälten, haben lediglich 17 (6,91\%) keine Angabe zu dieser Frage gemacht. Insgesamt gaben 70,1\% der Anwälte an, dass sie keine finanziellen Einbußen durch den Gang in die Mediation erlitten hätten. Lediglich $22,4 \%$ gaben an, mit der Mediation weniger verdient zu haben, als dies in einem streitigen Gerichtsverfahren der Fall gewesen wäre.

484 Dieses Ergebnis bestätigt sich auch, wenn man es auf die einzelnen Gerichtsbarkeiten herunter bricht, wobei die Aussagekraft der Ergebnisse sowohl für die Familien- und Verwaltungsgerichtsbarkeit als auch für die Sozialgerichtsbarkeit mit insgesamt 32 beantworteten Fragebögen eingeschränkt ist und kein statistisch repräsentatives Ergebnis wiedergeben kann.

485 Bei den Zivilgerichten gaben 69,11\% der insgesamt 246 Anwälte an, keine finanziellen Einbußen erlitten zu haben, während dies in 23,98\% der Fall war. 6,91\% der Befragten machten hierzu keine Angabe. Ähnlich stellt sich die Sachlage bei den Verwaltungsgerichten dar: Hier mussten 68,75\% der Anwälte keine Einbußen bei den Gebühren hinnehmen, 12,5\% hingegen schon; 18,75\% äußerten sich nicht zu der Frage. Beim Sozial- und beim Familiengericht stellt sich die Lage der Anwälte sogar noch positiver dar. Während am Familiengericht 70,0\% ihren vollen Gebührensatz bekommen haben, war das lediglich bei 20,0\% nicht der Fall; 10,0\% antworteten nicht auf diese Frage. Beim Sozialgericht schließlich waren es 78,13\% im Verhältnis zu 15,63\% der Befragten, die ihren vollen Gebührensatz bekommen haben. Hier enthielten sich lediglich 6,25\% einer Angabe.

486 Besonders bekräftigt wird die Annahme, durch die Mediation würden keine negativen finanziellen Anreize gesetzt, wenn man sich vor Augen führt, dass der größte Teil der befragten Anwälte eine Abrechnung nach der jeweiligen Gebührenordnung vorgenommen und nicht aufgrund einer selbstständigen Honorarvereinbarung auf Stundenbasis abgerechnet hat. Während von den insgesamt befragten 304 Anwälten 41 (13,5\%) keine Angaben machten, so haben 78,6\% nach der je- 
weiligen Gebührenordnung abgerechnet und nur 7,9\% trafen eine individuelle Gebührenvereinbarung.

Auch wenn dieses Gesamtergebnis eindeutig durch die Zivilgerichtsbarkeit beherrscht wird, stellt sich die Lage für alle Gerichtszweige ähnlich dar. Bei den Zivil- und Familiengerichten liegt der Anteil derjenigen, die über die Gebührenordnungen abrechen, bei etwa 80\% (Zivilgericht 81,3\%; Familiengericht 80,0\%), beim Sozial- und Verwaltungsgericht immerhin noch bei gut 60\% (Sozialgericht 65,63\%; Verwaltungsgericht 62,5\%). Entsprechend gering fallen die Quoten für diejenigen aus, welche eine Honorarvereinbarung getroffen haben. ${ }^{529}$ Die übrigen Befragten haben sich zu dieser Frage nicht geäußert. ${ }^{530}$

\section{Transaktionskostenersparnis}

Wie im Rahmen der ökonomischen Analyse angenommen, kann ein Einnahmeverlust, der aufgrund des Betreibens eines Verfahrens in der Mediation statt in einem streitigen Verfahren eingetreten ist, nicht durch Zeit- und Aufwandsersparnisse kompensiert werden. Die Quoten derjenigen, die dies bewerkstelligten bzw. derer, die dies nicht schafften, halten sich zwar in etwa die Waage, die Tendenz geht indessen dahin, dass ein solcher Ausgleich in Freizeit nicht geglückt ist (Frage 9a). So gaben von den 67 Anwälten, die sich zu dieser Frage äußerten, 58,2\% an, den Verdienstausfall nicht durch mehr Freizeit ausgeglichen zu haben, während dies im Gegenzug bei 41,8\% der Anwälte der Fall war.

\section{Zusammenfassung Gebührenordnung und Transaktionskosten- ersparnis}

Zusammenfassend lässt sich feststellen, dass die Gerichtsnahe Mediation insgesamt im Hinblick auf die Einnahmen der Rechtsanwälte keine negativen Anreize setzt, auch, wenn die Annahme der ökonomischen Analyse, für die Anwälte ergäben sich bezüglich ihrer Vergütung keinerlei negative Anreize, so nicht haltbar ist.

Wie die Datenauswertung gezeigt hat, überwiegt der Anteil der Anwälte, die nach dem Betreiben eines Verfahrens in der Mediation genauso gut stehen, als hätten sie das Verfahren vor dem gesetzlichen Richter durchgefochten, denjenigen der Anwälte, die Einkommensverluste hinnehmen mussten, deutlich. Darüber hinaus lässt sich feststellen, dass die Gebührenordnungen in ihrer derzeitigen Form geeignet erscheinen, die Mediation aus Anwaltssicht zumindest neutral beurteilen zu

\footnotetext{
529 Zivilgericht 6,91\%, Sozialgericht 12,5\%, Familiengericht 0,0\%, Verwaltungsgericht 18,75\%.

530 Zivilgericht 11,79\%, Sozialgericht 21,88\%, Familiengericht 20\%, Verwaltungsgericht 18,75\%.
} 
lassen. Der Großteil der Anwälte rechnete auch in der Mediation nach der entsprechenden Gebührenordnung ab und traf keine spezielle Honorarvereinbarung. Somit scheidet ein negativer Anreiz im Zusammenhang mit den Gebühren bei dem überwiegenden Anteil der Anwälte von vornherein aus, da es keinen Unterschied macht, ob sie ein Verfahren in der Mediation oder vor dem gesetzlichen Richter begleiten.

491 Für zumindest diejenige Hälfte des Teils der Anwaltschaft, der im Mediationsverfahren über geringere Einnahmen als im streitigen Verfahren verfügt, ergeben sich jedoch negative Anreize, als dass es ihnen nicht gelingt, die Einbußen an Gebühren durch mehr Freizeit wieder auszugleichen. Allerdings bleibt hier zu berücksichtigen, dass diese Anwälte die gewonnene Zeit für andere Einkünfte genutzt haben könnten.

\section{Reputation}

492 Die Annahmen der ökonomischen Analyse im Hinblick auf den Reputationsgewinn bzw. -verlust lassen sich durch die Auswertung der Datenerhebung nicht bestätigen. Weder erhoffen sich die Anwälte durch die Durchführung der Mediation einen Reputationsgewinn, noch befürchten sie auf der anderen Seite einen Reputationsverlust. Folglich ist die Reputation nicht geeignet, positive oder negative Anreize für den Anwalt zu bewirken.

493 Auf die Frage, ob der Reputationsgewinn für den Anwalt selbst oder seine Kanzlei ein Grund dafür wäre, den Mandanten auch in Zukunft eine Gerichtsnahe Mediation zu empfehlen, kreuzten 84,9\% der 304 befragten Anwälte diese Antwort nicht an. Lediglich für 10,5\% spielte der Reputationsgewinn für die Empfehlung der Mediation auch in Zukunft eine Rolle. 4,6\% der befragten Anwälte äußerten sich gar nicht zu der Frage, welche Gründe für sie für eine Weiterempfehlung der Mediation in Zukunft sprechen würden.

494 Auf der anderen Seite spricht jedoch nur für 3,0\% der Anwälte die Befürchtung eines negativen Images gegen eine Empfehlung der Mediation in Zukunft, während 67,4\% keinen negativen Effekt für ihr Ansehen befürchten. 29,6\% der Befragten äußerten sich gar nicht zu der Frage, was ihrer Ansicht nach gegen eine Empfehlung der Mediation in Zukunft sprechen würde.

495 Nichtsdestotrotz würde der größte Teil der Anwaltschaft den Mandanten die Mediation auch in Zukunft empfehlen, weil die Mehrzahl der Anwälte davon ausgeht, dass die Mandanten durch die Mediation geringeren psychischen Belastungen ausgesetzt sind und mit dem abschließenden Ergebnis zufriedener wären, als dies in einem gewöhnlichen streitigen Verfahren der Fall wäre. 
So würden 67,1\% der Anwälte die Mediation auch in Zukunft empfehlen, da sie sich hiervon eine größere Mandantenzufriedenheit erhoffen. Lediglich für 28,3\% der Anwälte spielt die Zufriedenheit ihrer Mandanten für die Weiterempfehlung der Mediation auch in Zukunft keine Rolle. 4,6\% der Anwaltschaft machten keine Angabe zur der Frage nach den Beweggründen für eine Weiterempfehlung der Mediation auch in Zukunft. Neben der generellen Zufriedenheit der Mandanten war für 51,0\% der Anwälte auch die in der Mediation angenommene geringere psychische Belastbarkeit ein Grund, die Mediation auch in Zukunft weiterzuempfehlen. Für 44,4\% spielte die geringere Belastung keine Rolle; 4,6\% machten auch hier wieder keine Angabe zu etwaigen Beweggründen.

Auch, wenn die eigentliche Intention der beratenden Anwälte demnach nicht die Mehrung der eigenen Reputation bzw. die Befürchtung um Einbußen derselbigen ist, so bringt die von den Anwälten angestrebte größere Zufriedenheit der eigenen Mandaten automatisch einen Reputationsgewinn und damit ein Beibehalten und ggf. sogar einen Zuwachs der Mandantenschaft mit sich. Demnach sind hier für die Anwälte, auch wenn dies nicht ihr primäres Anliegen ist, positive Anreize gegeben.

\section{Arbeitsmarktbedingungen und Gewinn von zusätzlicher Ausbil- dung und Erfahrung}

Dass die Tätigkeit des Anwalts in der Mediation dazu führt, dass er, wie in der ökonomischen Analyse bereits ausgeführt, sich in Verhandlungstechniken und führung fortbildet und im Hinblick auf eine etwaige Mediatorentätigkeit frühzeitig Einblicke in das Betätigungsfeld eines Mediators und in dessen Vorgehen und Technik nimmt, ist wohl unbestreitbar. Dennoch sind diese beiden Punkte für die wenigstens Rechtsanwälte ein Antrieb, ihren Mandanten auch in Zukunft die Durchführung eines Mediationsverfahrens nahe zu legen. So sahen lediglich 13,5\% der 304 befragten Anwälte in der Möglichkeit eines Ausbaus ihrer Verhandlungstechnik und -führung einen Grund in der Weiterempfehlung der Mediation. Für 81,9\% hingegen spielte dieser Beweggrund keinerlei Rolle. 4,6\% der Anwälte machten keine Angabe zu der Frage nach ihrem Beweggrund zur Weiterempfehlung der Mediation in der Zukunft.

Ähnlich verhält sich die Quote im Hinblick auf eine Weiterempfehlung der Mediation in Bezug auf eine eventuelle Mediatorentätigkeit. Hier waren es lediglich 8,9\% der Anwälte, die dies als Grund für eine Weiterempfehlung angaben, während dies für $86,2 \%$ keine Bedeutung hatte. Hier haben sich 4,9\% der Befragten nicht zu ihren Beweggründen geäußert. 


\section{Ergebnis Anreizstrukturen Anwälte}

500 In den meisten untersuchten Punkten ergeben sich für die Anwälte im Zusammenhang mit der gerichtsinternen Mediation weder positive noch negative Anreize, so dass sie dieser eher neutral gegenüberstehen. Da die Anwälte sich finanziell nach der Durchführung eines Mediationsverfahrens genauso gut stellen, wie dies bei einem streitigen Verfahren der Fall gewesen wäre, sind die Auswirkungen der Gebührenordnung daher als neutral bezüglich etwaiger Anreizstrukturen zu bezeichnen.

501 Auch, wenn sich die Rechtsanwälte durch die Mediation nicht direkt einen Reputationsgewinn erhoffen, so tritt dieser dennoch mit der von den Anwälten angestrebten größeren Zufriedenheit ihrer Mandanten mit dem erzielten Ergebnis ein. Insoweit ergeben sich hier durch einen Zuwachs an Reputation auch positive Anreize für die Anwaltschaft.

502 Auch eine Verbesserung der eigenen Verhandlungstechnik und -führung ist für die meisten Anwälte kein Anreiz, Verfahren in der Mediation zu betreiben, sodass sich auch hieraus keine positiven Anreize für die Rechtanwälte ergeben.

\section{Anreizstrukturen Parteien}

\section{Zeitlich bedingte finanzielle Anreize}

\section{a) Ersparnis an Arbeits- und Zeitaufwand}

503 Eine wirklich klare Bestätigung der Annahme, dass die Entscheidung für eine Mediation durch die Frage nach der möglichen Ersparung von Kosten motiviert wurde, lässt sich an Hand der empirischen Untersuchung nicht feststellen. Allenfalls eine vorsichtige Tendenz, die diese Annahme stützt, ist erkennbar. So war in 32,4\% der Fälle von insgesamt 404 retournierten Fragebögen auf die Frage, ob der Mediation zugestimmt worden sei, weil Arbeits- oder Zeitaufwand gespart werden sollte, mit „ja“ geantwortet worden. Immerhin noch $24,8 \%$ beantworteten diese Frage mit „eher ja“. Dem stehen die Parteien gegenüber, für welche die Ersparnis von Arbeits- und Zeitaufwand gar keine oder zumindest eher eine untergeordnete Rolle spielt. Während für 19,8\% eine etwaige Ersparnis an Arbeitszeit und der damit verbundene Zeitaufwand völlig belanglos für die Entscheidung für eine Mediation waren, hatte dieses Motiv für 17,8\% zumindest eine untergeordnete Bedeutung. 5,2\% äußerten sich nicht zu der Frage der Motivation im Zusammenhang mit Arbeits- und Zeitaufwandsersparnissen. 
Schlüsselt man dieses Ergebnis in die einzelnen Gerichtsbarkeiten auf, so ergeben sich unterschiedliche Ergebnisse:

Im Bereich der Zivilgerichtsbarkeit fällt die Einzelaufschlüsselung ähnlich aus, wie das bei der Gesamtbetrachtung der Fall war, was sicherlich zu einem Teil auch daran liegen kann, dass der Anteil der Fragebögen, die von der Zivilgerichtsbarkeit rückübersandt wurden, mit 240 Bögen der weitaus größte ist, sodass das Gesamtergebnis hierdurch maßgeblich beeinflusst wird. Während 9 Fragebögen (3,75\%) keinerlei Angaben zu der Frage enthielten, gaben 39,17\% an, dass die Einsparungen an Arbeit und Zeit sie dazu veranlasst hätten, sich für die Mediation anstelle des streitigen Gerichtsverfahrens zu entscheiden. Bei 25,83\% war diese Überlegung zumindest eher gegeben, als andere. Dagegen gaben 16,67\% an, dass dies eher weniger ausschlaggebend war, während dieses Kriterium für 14,58\% gar keine Bedeutung hatte.

Bei den anderen Gerichtsbarkeiten ist eine völlig andere Verteilung gegeben. So lässt sich bei den Sozialgerichten keinerlei Tendenz erkennen, da sich die Wahrscheinlichkeiten hier relativ gleich verteilen. ${ }^{531}$ Noch anders gestaltet sich die Situation bei den Familien- und den Verwaltungsgerichten. Im Rahmen der Familiengerichtsbarkeit scheinen die Aussichten auf eine Ersparnis an Arbeits- und Zeitaufwand eine eher untergeordnete Rolle zu spielen. Während 35,29\% angaben, dass dies für sie völlig bedeutungslos war, sahen zumindest 23,53\% in diesem Kriterium so gut wie keine Relevanz für ihre Entscheidung. Lediglich 11,76\% gaben an, dass die Möglichkeit einer Arbeits- und Zeitaufwandsersparnis zumindest eine untergeordnete Rolle für ihre Entscheidung für eine Mediation gespielt hat, während dies für 17,65\% der Hauptgrund war. Darüber hinaus machten im Rahmen der Familiengerichtsbarkeit 11,76\% der Befragten keinerlei Angaben zu dieser Frage.

Auch bei den Verwaltungsstreitigkeiten ist eine mögliche Ersparnis von Arbeitsund Zeitaufwand kein Kriterium, was die Leute dazu bewegt, sich für eine Mediation zu entscheiden. So war für 31,37\% eindeutig dieser Aspekt für die Entscheidung für das Betreiben eines Mediationsverfahrens gänzlich ohne Belang, für $15,69 \%$ immerhin nur eine marginale Bedeutung. Für immerhin 27,45\% war dieser Gesichtspunkt immerhin von untergeordneter Bedeutung, während 15,69\% ihn als Hauptkriterium für die Entscheidung für eine Mediation angaben. 9,80\% machten keine Angabe zu diesem Beweggrund.

531 27,08\% ja, 22.92\% eher ja, 20,83\% eher nein, 23,96\% nein, 5,21\% keine Angabe. 
508 Zusammenfassend lässt sich also festhalten, dass einzig in der Zivilgerichtsbarkeit die Aussicht auf die Ersparnis an Arbeitszeit einen Anreiz im Hinblick auf die Mediation darstellt. In der Sozialgerichtsbarkeit lässt sich keine genaue Aussage über die Relevanz dieses Kriteriums treffen, da hier alle Entscheidungsmöglichkeiten nahezu paritätisch verteilt sind. Im Bereich der Familien- und Verwaltungsgerichtsbarkeit hingegen spielen Zeit- und Arbeitsersparnisse eher eine untergeordnete Rolle.

\section{b) Ersparnis an Geld}

509 Auch das Kriterium der möglichen Ersparnis an Geld scheint in vielen Fällen nicht der Hauptgesichtspunkt gewesen zu sein, warum die Parteien sich für eine Mediation entschieden haben. Für den größten Teil der Befragten spielten finanzielle Aspekte gar keine oder nur eine marginale Rolle bei der Frage nach der Entscheidung für eine Mediation. So gaben 31,2\% der Befragten an, dass finanzielle Aspekte ihre Entscheidung überhaupt nicht beeinflusst hätten. Bei 24,3\% waren finanzielle Aspekte zumindest von marginaler Bedeutung. Lediglich für 21,8\% waren die möglichen finanziellen Einbußen das entscheidende Kriterium, warum sie den Weg der Mediation dem des streitigen Verfahrens vorgezogen haben. Bei 16,8\% waren die Kostengesichtspunkte zumindest ein Teilaspekt bei der Entscheidung für die Mediation. 5,9\% der 404 Befragten machten keine Angabe darüber, inwieweit die Kosten für sie entscheidend waren.

510 Noch deutlicher wird das Ergebnis bei einer Aufschlüsselung in die einzelnen Gerichtsbarkeiten. Besondern im Bereich der Sozial- und der Verwaltungsgerichtsbarkeit wird hier deutlich, dass es den Beteiligten bei der Entscheidung für eine Mediation in den meisten Fällen nicht darum geht, Geld zu sparen. So gaben $38,54 \%$ bei den sozialgerichtlichen und $37,25 \%$ bei den verwaltungsgerichtlichen Fällen an, dass Kostengesichtspunkte für sie kein Entscheidungsgrund gewesen wären. 29,17\% bzw. 29,41\% gaben an, dass finanzielle Gesichtspunkte ihre Entscheidung wohl eher nicht beeinflusst hätten. Auf der anderen Seite waren für nur 12,50\% bei den Sozialgerichten und sogar nur 7,84\% bei den Verwaltungsgerichten die Kosten der Grund warum sie sich für eine Mediation entschieden hatten. Bei 13,54\% bzw. 15,69\% haben die Kosten wohl eher eine Rolle gespielt als andere Gesichtspunkte. 6,25\% bei den Sozialgerichten sowie 9,80\% bei den Verwaltungsgerichten machten keinerlei Angabe zu der Frage.

511 Bei den Familiengerichten sind die Verhältnisse relativ ausgeglichen. Während für 29,41\% der Kostenaspekt der Anreiz für eine Mediation war, war diese für ebenfalls 29,41\% völlig belanglos. Während 23,53\% angaben, dass Kostengesichtspunkte schon eine gewisse Rolle für ihre Entscheidung gespielt haben, gaben hin- 
gegen 11,76\% der Befragten an, dass Kostensaspekte eher unwichtig waren. 6,25\% äußerten sich im Rahmen der Familiengerichtsbarkeit nicht zu dieser Frage.

Eine ähnliche Verteilung ergibt sich auch für die Zivilgerichte. Der Anteil derjenigen, die sich aus Kostengründen für die Mediation entschieden haben und der jener, für die Kostenaspekte keinerlei Relevanz im Zusammenhang mit der Entscheidung für eine Mediation aufweisen, halten sich auch hier etwa die Waage. So gaben 27,92\% an, dass sie sich aus Kostengründen für die Mediation entschieden hätten, währen dies bei 27,08\% gerade nicht der Fall gewesen ist. Auch die Wahrscheinlichkeiten für diejenigen, bei denen Kostenaspekte zumindest eine gewisse Rolle spielten als auch für diejenigen, bei denen dies nur eine ganz untergeordneter Punkt war verhalten sich etwa gleich (17,92\% „eher ja“, 22,08\% „eher nein“). 5,0\% machten im Rahmen der Zivilgerichtsbarkeit keine Angabe zu dieser Frage.

Demnach hat sich die Annahme aus der ökonomischen Analyse durch die empirische Untersuchung nicht bestätigt, dass die Ersparnis an finanziellen Mitteln einen großen Einfluss auf die Entscheidung für eine Mediation statt des gewöhnlichen streitigen Verfahrens hat.

\section{c) Zeitliche Ersparnis}

Was jedoch für die meisten Befragten von zentraler Bedeutung für eine Entscheidung für den Weg in die Mediation war, ist die mit der Mediation verbundene Zeitersparnis. Dieser Beweggrund spiegelt sich nicht nur in der Gesamtauswertung wieder, sondern lässt sich auch in den einzelnen Gerichtsbarkeiten wieder finden.

In der Gesamtauswertung gaben 64,4\% der Befragten an, dass die Zeitersparnis einer der Hauptgründe für sie gewesen wäre, sich für eine Mediation zu entscheiden. Bei immerhin noch 24,5\% war diese Zeitersparnis ebenfalls, wenn auch nicht einer der Hauptgründe, so aber noch ein Beweggrund von eher zentraler Bedeutung für den Gang in die Mediation. Lediglich 5,0\% gaben an, dass Zeitersparnis für sie eine eher untergeordnete Rolle spiele, während lediglich 3,2\% in der Zeitersparnis gar keinen Grund für die Anstrengung eines Mediationsverfahrens sahen. 3,0\% äußerten sich nicht zu der Frage, wie wichtig ihnen eine Zeitersparnis bei der Entscheidung für die Mediation gewesen ist.

Noch etwas ausgeprägter als in der Gesamtauswertung ist das Einzelergebnis für die Zivilgerichtsbarkeit. Hier entschieden sich $71,25 \%$ für eine Mediation, weil sie sich davon eine Zeitersparnis im Vergleich zum streitigen Verfahren erhofften. Für weitere 20,0\% stellte die Zeitersparnis zwar nicht den alleinigen Beweggrund, aber dennoch ein eher wichtiges Kriterium bei der Entscheidung für die Mediation dar. Für allein 5,42\% war die Zeitersparnis nur von untergeordneter Bedeu- 
tung, während bei 1,25\% dieser Aspekt nichts mit der Entscheidung für den Gang in die Mediation zu tun hatte.

517 Ähnliche Wahrscheinlichkeitsverteilungen, wenn auch nicht ganz so ausgeprägt, finden sich in den anderen Gerichtszweigen. Für den Großteil der Befragten war die Zeitersparnis das ausschlaggebende Kriterium bei der Wahl der Mediation (Sozialgericht 58,33\%; Familiengericht 58,82\%; Verwaltungsgericht 45,10\%). Für einen weiteren großen Prozentsatz der Befragten war die kürzere Verfahrensdauer zumindest einer der ausschlaggebenden Punkte (Sozialgericht 28,13\%; Familiengericht 29,41\%, Verwaltungsgericht 37,25\%). Dementsprechend niedrig sind die Quoten bei denjenigen Befragten ausgefallen, die angegeben haben, dass Zeitersparnis nur marginal ihre Entscheidung beeinflusst hat bzw. für die Entscheidung gänzlich ohne Belang war („eher nein“: Sozialgericht 1,04\%; Familiengericht 5,88\%; Verwaltungsgericht 9,80\%; „nein“: Sozialgericht 7,29\%, Familiengericht 5,88\%; Verwaltungsgericht 3,92\%).

518 Folglich lässt sich festhalten, dass für die Verfahrenbeteiligten einer der Hauptentscheidungsgrund für den Weg in die Mediation in der Annahme dessen besteht, dass die Verfahrensdauer im Verhältnis zu der eines streitigen Verfahrens wesentlich kürzer sei.

\section{d) Zeitersparnis nach dem Verfahren}

519 Auch nach der Durchführung des Mediationsverfahrens geben die meisten Parteien noch an, dass sie aufgrund der Mediation anstatt der Durchführung eines streitigen Verfahrens eine Zeitersparnis hatten; auch, wenn hier die Quoten nicht mehr ganz so positiv ausfallen, wie dies bei der Frage nach einer Zeitersparnis als Beweggrund für die Entscheidung für eine Mediation der Fall war.

520 Nach der Gesamtauswertung gaben hier 47,5\% der Befragten an, dass sie durch die Durchführung eines Mediationsverfahrens im Vergleich zum streitigen Verfahren Zeit eingespart hätten. Noch 22,0\% gaben an, dass sie wohl eher Zeit gespart, als verloren haben. Demgegenüber gehen 17,1\% davon aus, dass das Mediationsverfahren nicht kürzer gedauert hätte, als ein streitiges Verfahren. 9,4\% sind der Überzeugung, dass das Mediationsverfahren wohl eher nicht schneller war, als der streitige Prozess.

521 Bei einer Einzelaufschlüsselung in die jeweiligen Gerichtszweige zeigt sich, dass der überwiegende Teil im Bereich der Zivil- und Sozialgerichtsbarkeit auch noch nach der Mediation von einer Zeitersparnis ausgeht. Bei den Familien- und Verwaltungsgerichten hingegen ist der Großteil der Parteien nun der Ansicht, dass das streitige Verfahren auch nicht länger gedauert hätte, als dies bei der Mediation der Fall war. 
So gaben bei den zivilgerichtlichen Verfahren 56,25\% an, dass sie Zeit durch die Mediation gespart hätten, während immerhin noch 21,25\% davon ausgehen, dass sie wohl eher Zeit gespart haben. Auf der anderen Seite sind 12,92\% der befragten Parteien der Ansicht im Vergleich zum streitigen Verfahren keinerlei Zeit eingespart zu haben, während 6,25\% der Ansicht sind, eher weniger Zeit gespart zu haben.

Ähnlich verteilen sich die Wahrscheinlichkeiten bei den sozialgerichtlichen Fällen. Hier gaben 42,71\% der befragten Parteien an, durch das Mediationsverfahren einen Zeitvorteil bekommen zu haben, während 23,96\% immerhin noch der Ansicht waren, eher Zeit gespart zu haben. Auf der anderen Seite waren 12,50\% der Überzeugung, dass sie mit einem streitigen Verfahren ebenso schnell zu einem Ergebnis gekommen wären, wie dies durch die Mediation der Fall war; 15,63\% gingen eher davon aus, keine Zeit gespart zu haben.

Bei den Familien- und Verwaltungsgerichten hingen ist der überwiegende Teil der Befragten der Ansicht, durch die Mediation keinerlei Zeitvorteile erreicht zu haben. So sind $41,18 \%$ bei den Familien- und immerhin noch 37,25\% bei den Verwaltungsgerichten der Auffassung, dass ein streitiges Verfahren ebenso schnell abgeschlossen worden wäre. Jeweils weitere 11,76\% der Parteien waren der Auffassung, eher keine Zeit gespart zu haben, als dass ihnen die Mediation irgendeinen zeitlichen Vorteil eingebracht hätte. Davon sind bei den Familiengerichten lediglich 17,65\% und bei den Verwaltungsgerichten 23,53\% der Parteien ausgegangen. Für jeweils 23,53\% war auch bei diesen Gerichtszweigen die Mediation eindeutig mit einer Zeitersparnis verbunden.

Zusammenfassend lässt sich damit festhalten, dass im Bereich der Zivil- und der Sozialgerichtsbarkeit positive Anreize dadurch geschaffen werden, dass der Großteil der Parteien auch nach dem Prozess noch der Ansicht ist, dass ihnen das Mediationsverfahren im Vergleich zu einem streitigen Prozess einen Zeitvorteil eingebracht hat. Bei den Familien- und Sozialgerichten empfindet hingegen jeweils eine knappe Mehrheit keine spürbaren zeitlichen Vorteile durch die Anspruchnahme der Mediation. Dennoch sollte in diesem Zusammenhang nicht außer betracht gelassen werden, dass es sich hierbei wirklich nur eine knappe Mehrheit handelt, sodass die knappe Hälfte der beteiligten Parteien die Mediation als eine im Vergleich zum streitigen Verfahren zeitsparende Alternative betrachtet.

\section{e) Kostenersparnis nach dem Verfahren}

Ähnlich wie die Gesamtbetrachtung bei der Frage nach dem ersparten Zeitaufwand bei einem Mediationsverfahren im Vergleich zu einem streitigen Prozess, ergeben sich positive Anreize für die Mediation auch im Hinblick auf die Kosten, 
die den Parteien in einem Mediationsverfahren im Vergleich zum streitigen Verfahren entstehen. Der überwiegende Teil der befragten Parteien, nämlich 41,1\%, gehen von einer Kostenersparnis durch die Mediation aus. Immerhin noch 20,0\% sind der Auffassung, dass sie durch das Mediationsverfahren eher Geld gespart haben, als dass es sie mehr gekostet hätte als ein streitiger Prozess. Demnach gehen nach der Gesamtbetrachtung 61,1\% der Parteien von einer Kostenersparnis aus. Demgegenüber stehen 31,6\%, nach deren Ansicht das Mediationsverfahren nicht weniger gekostet hat, als dies bei einem normalen streitigen Prozess der Fall gewesen wäre. 15,3\% gehen hierbei davon aus, dass das Mediationsverfahren definitiv nicht günstiger für war, während 16,3\% eher davon ausgehen, dass das Mediationsverfahren nicht günstiger war. 7,2\% der insgesamt 404 Befragten machten keine Angabe zu der gestellten Frage.

527 In etwa gleich verteilen sich die Quoten im Bereich der Zivilgerichtsbarkeit. Auch hier werden die Wahrscheinlichkeiten immer geringer, je mehr die Parteien angenommen haben, dass die Unkosten, die durch ein Mediationsverfahren verursacht wurden, nicht geringer sind als bei einem streitigen Verfahren. So waren 45,83\% der Überzeugung, dass ihr Mediationsverfahren günstiger war, als dies bei einem streitigen Prozess der Fall gewesen wäre. Immer noch 24,58\% gehen davon aus, dass die Mediation eher günstiger als teurer im Vergleich zu einem normalen Verfahren war. Den umgekehrten Fall, nämlich dass die Mediation sie nicht weniger gekostet hat als ein streitiges Verfahren, nehmen nur noch 12,5\% an, während lediglich 9,17\% von mindestens gleichen Kosten der beiden Alternativen ausgingen.

528 Auch bei den anderen Gerichtszweigen geht der überwiegende Anteil der Befragten davon aus, dass die Mediation weniger kostenintensiv war. ${ }^{532}$ Die Verteilung der übrigen Wahrscheinlichkeiten divergiert bei den einzelnen Gerichtsbarkeiten allerdings und weicht damit von der Gesamtaufschlüsselung ab. Während im Bereich der Sozialgerichtsbarkeit lediglich 11,46\% annehmen, dass das Mediationsverfahren eher günstiger war als ein entsprechender Prozess, nehmen hier jeweils 25,0\% an, dass die Mediation eher nicht günstiger bzw. dass sie in keinem Fall günstiger war. Damit ist das Sozialgericht die einzige Gerichtsbarkeit, bei der die Mehrheit davon ausgeht, dass sie keine Kosten durch ein Mediationsverfahren gespart hätte (6,25\% machten hierzu keine Aussage).

529 Bei den Familien- und Verwaltungsgerichten gehen noch 11,76\% bzw. 17,65\% davon aus, dass die Mediation eher günstiger war als ein streitiger Prozess. Damit ist auch hier, ebenso wie bei den Zivilgerichten, über die Hälfte der Befragten der

532 Sozialgericht 32,29\%; Familiengericht 47,06\%; Verwaltungsgericht 33,33\%. 
Auffassung, dass für sie die Mediation eine Kostenersparnis gebracht hat. Im Bereich der Familiengerichtsbarkeit sind darüber hinaus 11,76\% der Ansicht, dass die Mediation für sie eher nicht günstiger war und 17,65\% sind der Auffassung dass die Mediation definitiv nicht günstiger war als der Weg über das streitige Verfahren. Bei den Verwaltungsgerichten sind 25,49\% der Auffassung, dass die Mediation keine Kostenersparnis bedeutet, während 19,61\% eher nicht an eine Kostenersparnis glauben. Bei den Familiengerichten äußerten sich 11,76\%, bei den Verwaltungsgerichten 3,92\% nicht zu der Frage.

Abschließend lässt sich sagen, dass im Großen und Ganzen die Mediation dadurch für die Parteien im Vergleich zu einem streitigen Verfahren attraktiv ist, weil sie für die einzelnen Parteien in den meisten Fällen eine Kostenersparnis bedeutet. Insoweit ist hier ein positiver Anreiz gegeben.

\section{f) Ersparnis sonstiger Kosten}

\section{aa) Transaktionskosten}

Die im Rahmen der ökonomischen Analyse aufgestellte Annahme, dass die durch die Mediation verursachten Transaktionskosten geringer seien, als dies im streitigen Verfahren der Fall ist, lässt sich, zumindest für einzelne Gerichtsbarkeiten, ebenfalls durch die empirische Untersuchung belegen. So war für viele Parteien, die sich für die Mediation entschieden haben, die Annahme einer geringeren Belastung als im streitigen Verfahren ausschlaggebend. So gaben 42,1\% der befragten Parteien als Motivation für die Teilnahme an einem Mediationsverfahren an, dass sie gehofft hatten, dass das Mediationsverfahren weniger belastend sei als ein gewöhnlicher streitiger Prozess. Weitere 23,8\% gaben an, dass dieser Grund sie eher zu einer Teilnahme an einer Mediation bewogen hätte, als andere. Für lediglich 15,3\% war die mögliche geringere Belastung kein Entscheidungskriterium und für 15,1\% spielte diese Annahme einer eher untergeordnete Rolle. 3,8\% der insgesamt 404 beantworteten Fragebögen enthielten keine Antwort auf eine mögliche Motivation durch geringere Belastungen. Bei dieser Gesamtauswertung ist jedoch zu beachten, dass sie sehr stark durch die Zivilgerichtsbarkeit geprägt ist. Während von hier für die Auswertung 240 Fragebögen zur Verfügung standen, waren dies bei der Sozialgerichtsbarkeit nur 96, bei der Verwaltungsgerichtsbarkeit lediglich und 51 und bei den Familiengerichten sogar nur 17 Fragebögen. Hieraus lässt sich auch schon ersehen, dass zumindest im Bereich der Familiengerichtsbarkeit aufgrund der zu geringen Zahl der auszuwertenden Fragebögen keine gesicherten statistischen Ergebnisse präsentiert, sondern allenfalls vorsichtige Tendenzen widergespiegelt werden können. 
532 Bei einer Aufschlüsselung in die einzelnen Gerichtszweige stellt sich das Ergebnis, zumindest im Bereich der Zivil- und Familiengerichtsbarkeit noch ausgeprägter dar. In beiden Fällen gaben über 50\% der Parteien an, dass die geringere Belastung die ausschlaggebende Motivation für ein Mediationsverfahren war (Zivilgerichte 52,5\%; Familiengerichte 58,82\%). Ein weiterer großer Teil der befragten Parteien gab an, dass die geringere Belastung zumindest ein wesentlicher Punkt im Zusammenhang mit der Entscheidung für ein Mediationsverfahren war (Zivilgerichte 25,83\%; Familiengerichte 29,41\%). Irrelevant war die Überlegung nach einer Belastungsersparnis lediglich für 7,08\% bei den Zivilgerichten und 11,76\% in der Familiengerichtsbarkeit. Während bei letzteren niemand angab, dass diese Kriterium eher eine untergeordnete Rolle spielt, so war dies bei der Zivilgerichtsbarkeit doch immerhin bei 11,25\% der Fall. Keine Angaben zu dieser Frage machten bei den Zivilgerichten 3,39\%. Bei den Familiengerichten enthielten alle Fragebögen Antworten auf diese Frage.

533 Ausgeglichener gestaltet sich die Wahrscheinlichkeitsverteilung im Bereich der Sozial- und Verwaltungsgerichtsbarkeit. So gaben bei den Sozialgerichten 23,96\% an, dass die geringere Belastung für sie der Hauptgrund für den Weg der Mediation war, während dies bei $20,83 \%$ der Befragten noch von übergeordneter Bedeutung war. Im Gegensatz dazu gaben 23,95\% an, dass eine Ersparnis an Belastungen für sie bei der Entscheidung eher unwichtig gewesen ist und bei 26,04\% war die geringere Belastung gar kein Entscheidungskriterium. 5,21\% haben auf diese Frage keine Antwort gegeben.

534 Ähnlich verhält es sich bei den Verwaltungsgerichten, auch wenn hier mit 35,29\% der größte Teil der Parteien angab, sich nicht wegen einer geringeren Belastbarkeit für eine Mediation entschieden zu haben. Für weitere 21,57\% war dies ebenfalls nur von untergeordneter Bedeutung. Im Gegensatz dazu waren es immerhin 21,57\% der befragten Parteien, die sich wegen der Erwartung einer geringeren Belastung auf ein Mediationsverfahren eingelassen haben, während dies 17,57\% zumindest noch als einen überwiegenden Aspekt in ihre Entscheidung haben mit einfließen lassen. Bei der Verwaltungsgerichtsbarkeit machten 3,92\% keine Angaben zu dieser Frage.

535 Insoweit stellt sich zumindest im Rahmen der Zivil- und Familiengerichte nach der statistischen Auswertung eine gerichtsinterne Mediation insofern als sinnvolle Alternative zu einem streitigen Gerichtsverfahren dar, als dass die Parteien hoffen, durch die Mediation weniger belastet zu werden, als dies bei einem streitigen Prozess der Fall wäre. Dementsprechend entscheiden sich die Parteien zumindest in diesen Gerichtsbarkeiten für diesen Weg, um ihre Transaktionskosten zu senken. Im Bereich der Sozial- und Verwaltungsgerichte sind es etwa $40 \%$ die sich von einer Mediation geringere Belastungen versprechen und dies zumindest als Teilas- 
pekt in ihre Entscheidung für eine Mediation haben einfließen lassen. Auch, wenn der überwiegende Teil sich aus anderen Beweggründen für eine Mediation entschieden hat, so hat die Ersparnis von Transaktionskosten hier dennoch ein recht großes Gewicht, sodass sich auch hieraus für einige Parteien eine Anreizfunktion ergibt.

\section{bb) Erhalt der Parteibeziehungen}

Auch der Erhalt langfristiger Geschäftsbeziehungen oder auch persönlicher Verbindungen kann einen Anreiz darstellen, sich auf eine konsensuale Streitbeilegung einzulassen, da die Parteien größere Chancen sehen, diese Beziehungen bei einer Mediation aufrechtzuerhalten als dies bei einem streitigen Prozess wäre.

Im Rahmen der empirischen Untersuchung ließ sich feststellen, dass es tatsächlich mehr als der Hälfte (17,6\% sehr wichtig; 34,2\% ziemlich wichtig) der befragten Parteien wichtig ist, die gute Beziehung zur anderen Partei aufrecht zu erhalten. Auf der anderen Seite sahen 46,0\% das Aufrechterhalten der Beziehung als weniger wichtig an (30,2\% nicht sehr wichtig; 15,8\% vollkommen unwichtig). 2,2\% der 404 Parteien, die Fragebögen abgegeben haben, antworteten nicht auf diese Frage.

Erstaunlich ist hierbei, dass bei einer Einzelbetrachtung der Gerichtszweige zwar im Bereich der Sozial-, der Familien- und der Verwaltungsgerichte die Parteibeziehungen eine gewichtige Rolle spielen, dass im Rahmen der Zivilgerichtsbarkeit die Aufrechterhaltung einer guten Beziehung zur anderen Partei im Großen und Ganzen als eher unwichtig angesehen wird. Dies gaben 60,83\% (23,75\% vollkommen unwichtig; 37,08\% nicht sehr wichtig) der 240 befragten Parteien an. Demgegenüber stehen lediglich 36,66\% (12,08\% sehr wichtig; $24,58 \%$ ziemlich wichtig), die an einer Aufrechterhaltung einer guten Beziehung zu der anderen Partei ein Interesse haben. 2,50\% der befragten Parteien im Zivilprozess äußerten sich nicht zu der Frage nach der Wichtigkeit eines Aufrechterhaltens der Beziehungen.

Genau konträr verhalten sich die Statistiken in den anderen drei Gerichtsbarkeiten. Hier sind die Beziehungen zur anderen Partei durchaus von einiger Bedeutung. So gaben bei den Sozialgerichten 76,04\% (30,21 „sehr wichtig“; 45,83\% „ziemlich wichtig“"), bei den Verwaltungsgerichten 70,59\% (17,65\% ,sehr wichtig“; $52,94 \%$,ziemlich wichtig“) und bei den Familiengerichten ebenfalls 70,59\% (23,53\% „,sehr wichtig“; 47,06\% „ziemlich wichtig“) der Parteien an, dass ihnen an der Aufrechterhaltung ihrer Beziehungen zur anderen Partei gelegen sei. Das familiengerichtliche Ergebnis darf an dieser Stelle jedoch nicht überinterpretiert werden, da es lediglich auf einer Gesamtsumme von 17 Fragebögen beruht, von denen zwei kein Angabe zu der Wichtigkeit der Parteibeziehung enthielten, was einer 
Quote von 11,76\% entspricht. Bei den Sozialgerichten liegen der Untersuchung 96 (keine Angabe bei 12,5\%) und bei den Verwaltungsgerichten immerhin noch 51 (keine Angabe bei 15,69\%) Fragebögen zugrunde. Den restlichen befragten Parteien war die Aufrechterhaltung der guten Beziehung zur anderen Partei weniger wichtig. 533

540 Trotz dieses Ergebnisses, dass über die Hälfte der Parteien an einer Aufrechterhaltung der Parteibeziehungen gelegen ist, gibt ebenfalls über die Hälfte der Befragten an, dass die zur anderen Partei bestehende Beziehung für sie leicht zu ersetzen ist $(22,3 \%$,sehr leicht“; 28,5\% „eher leicht"). Auf der anderen Seite sind es nur $39,6 \%$, die den Abbruch einer Beziehung nur schwer oder gar überhaupt nicht (22,5\% „eher schwer“; $17,1 \%$,überhaupt nicht“) ersetzen könnten. 9,7\% der befragten 404 Parteien machten zu dieser Frage keine Angabe.

541 Bei einer Aufgliederung in die einzelnen Gerichtszweige, ergibt sich ein sehr unterschiedliches Bild. Während die Parteibeziehungen im Bereich der Zivil- und Familiengerichte leicht zu ersetzen zu sein scheinen, ist dies im Bereich des Sozialgerichtes nicht der Fall. Beim Verwaltungsgericht ergibt sich eine recht ausgeglichene Wahrscheinlichkeitsverteilung.

542 Von den 240 Parteien (7,08\% machten keine Angaben zu der Frage der Ersetzbarkeit der Parteibeziehung), die den Fragebogen bei den Zivilgerichten ausfüllten gaben 60,0\%\% (29,17\% ,sehr leicht“; 30,83\% „eher leicht" ${ }^{\circ /}$ an, dass sie die Beziehung zu der anderen Partei leicht substituieren könnten. Den übrigen 32,92\% fällt dies schwer $(21,67 \%)$ oder ist dies gar unmöglich $(11,25 \%)$.

543 Für die Familiengerichtsbarkeit ergibt sich, dass die Parteien hier zu 52,94\% $(17,65 \%$,sehr leicht“; 35,29\% „,eher leicht“) den Verlust der Beziehung zu der anderen Partei ersetzen können. Weiteren 35,3\% (17,65\% „überhaupt nicht“; $17,65 \%$,eher schwer") gelingt dies nicht ohne weiteres oder sogar gar nicht. 11,76 der insgesamt 17 Fragebögen enthielten keine Antwort auf die gestellte Frage. Wie im Rahmen der gesamten Untersuchung ist im Zusammenhang mit der Familiengerichtsbarkeit die geringe Gesamtzahl der der Untersuchung zugrunde liegenden Fragebögen zu berücksichtigen, sodass die angegebenen Wahrscheinlichkeiten nur dazu dienen können, einen Trend anzuzeigen.

533 Sozialgericht 20,84\%, aufgeteilt in 4,17\% ,vollkommen unwichtig“; $16,67 \%$,nicht sehr wichtig“; Familiengericht 29,41\%, aufgeteilt in 5,88\% ,vollkommen unwichtig“; 23,53\% ,nicht sehr wichtig“; Verwaltungsgericht 29,41\%, aufgeteilt in 3,92\% ,vollkommen unwichtig“; 25,49\% ,nicht sehr wichtig“. 
Bei den Sozialgerichten, deren Untersuchung eine Gesamtzahl von 96 Fragebögen zugrunde liegt, von denen jedoch 12,5\% keine Antwort auf die Frage nach der Substituierbarkeit der Parteibeziehung enthalten, ist es für den Großteil der befragten Parteien schwieriger (29,17\%) oder gar unmöglich (27,08\%), eine zerstörte Beziehung zur anderen Partei durch andere zu ersetzen. Dem stehen 31,26\% (9,38\% „sehr leicht“; $21,88 \%$ „eher leicht“) der Parteien gegenüber, für die dies möglich ist.

Der verwaltungsgerichtlichen Untersuchung schließlich liegen insgesamt 51 Fragebögen zugrunde, bei denen 15,69\% keine Angaben im Hinblick auf die Ersetzbarkeit der Parteibeziehung enthalten. Daneben ist es 41,18\% (25,49\% „überhaupt nicht“; 15,69\% ,eher schwer") der befragten Parteien eher schwer oder gar nicht möglich eine Parteibeziehung zu substituieren, während dies bei 43,14\% (15,69\% „,sehr leicht“; 27,45\% ,eher leicht") der Fall ist.

Im Rahmen der Nachbefragung der Parteien ergab sich, dass sich das Verhältnis derjenigen Parteien, die nach Abschluss der Mediation das Verhältnis zur anderen Partei aufrecht erhalten haben, zu denjenigen, die keine Beziehung mehr zu der anderen Partei unterhalten, in etwa die Waage hält. So geben 44,8\% der $154 \mathrm{Be}-$ fragten an, dass die Beziehung noch besteht, während 47,2\% dies verneinen. 8,0\% gaben keine Angabe über das Fortbestehen der Parteibeziehungen.

Erstaunlich dabei ist, dass die Wahrscheinlichkeitsaufteilungen in den einzelnen Gerichtsbarkeiten zum Teil stark divergieren. Während bei den Sozialgerichten von den 29 befragten Parteien 65,52\% und bei den Verwaltungsgerichten von den 39 Befragten 56,41\% angaben, die Beziehungen zur anderen Partei aufrecht erhalten zu haben, ist dies im Bereich der Zivilgerichte wesentlich seltener der Fall. Hier beträgt die Quote der aufrechterhaltenen Beziehungen lediglich 33,33\% bei insgesamt 93 Befragten. Demgegenüber gaben 59,14\% an, die Beziehungen zur anderen Partei abgebrochen zu haben. Entsprechend verhalten sich die anderen Wahrscheinlichkeiten: Während bei den Zivilgerichten 33,33\% angaben, die Beziehungen zur anderen Partei aufrecht erhalten zu haben, gaben bei der Sozialund der Verwaltungsgerichtsbarkeit jeweils etwa ein Drittel der Befragten an, die Beziehungen abgebrochen zu haben (Sozialgericht 31,03\%; Verwaltungsgericht 31,71\%). Bei den Zivilgerichten äußerten sich 7,53\%, beim Sozialgericht 3,45\% und beim Verwaltungsgericht 12,2\% nicht zu der gestellten Frage.

Durch diese unterschiedlichen Ergebnisse lässt sich aufgrund der statistischen Untersuchung keine generelle Aussage darüber treffen, ob die Mediation wirklich dazu geeignet ist, die Parteibeziehungen zu schonen. Jedoch sind hier Tendenzen erkennbar, dass dies im Bereich der Sozial- und der Verwaltungsgerichte durchaus 
der Fall ist, während im Bereich der Zivilgerichtsbarkeit die Mediation eher nicht dazu führt, dass die Parteibeziehungen intakt bleiben.

\section{cc) Vermeidung von Folgeprozessen}

549 Die in der ökonomischen Analyse gemachte Annahme, dass die Mediation ein Anreiz zur Vermeidung von Folgeprozessen ist, lässt sich durch die empirische Untersuchung voll umfänglich, durch alle Gerichtsbarkeiten hindurch, bestätigen. So gaben in der Gesamtauswertung 79,5\% (53,5\% ,ja“; 26,0\% ,eher ja“) der 404 befragten Parteien an, dass die Motivation für den Gang in eine Mediation in der Hoffnung bestand, den Konflikt dauerhaft zu lösen, sodass es zu keinerlei Folgekonflikten kommt. Dem stehen nur 17,6\% gegenüber für die dies eher von untergeordneter bzw. keinerlei Relevanz war (10,4\% „eher nein“; 7,2\% „nein“). 3,0\% der 404 Befragten machten hier keinerlei Angaben.

550 Wie bereits angedeutet spiegelt sich dieses Ergebnis auch in den Einzelaufschlüsselungen der vier untersuchten Gerichtszweige wider. So war das Kriterium der Folgeprozessvermeidung bei den Zivilgerichten in 81,66\% (54,58\% ,ja“; 27,08\% „,eher ja“), bei den Sozialgerichten in 66,67\% (42,71\% ,ja“; 23,96\% „,eher ja“), bei den Familiengerichten in 94,12\% (76,47\% ,ja“; $17,65 \%$ „eher ja“) und bei den Verwaltungsgerichten in 88,23\% (60,78\% ,ja“; $27,45 \%$ „eher ja“) ausschlaggebend für die Entscheidung für eine Mediation. Entsprechend gering stellen sich die Wahrscheinlichkeitsverteilungen für die Fälle dar, in denen die Folgeprozessvermeidung von eher untergeordneter bis hin zu gar keiner Bedeutung sind. ${ }^{534}$ Bei den Zivilgerichten machten 2,92\% der Befragten, bei den Sozialgerichten 4,17\%, bei den Familiengerichten 0,0\% und bei den Verwaltungsgerichten 1,96\% keine Angaben.

\section{Endgültigkeit des Mediationsverfahrens}

551 Die im Rahmen der ökonomischen Analyse des Mediationsverfahrens aufgestellte Annahme, dass die Mediation sich trotz Scheiterns positiv auf den weiteren Verhandlungsablauf auswirkt, lässt sich durch die empirischen Erhebungen zumindest vorsichtig bestätigen. An einer ausdrücklichen Bestätigung oder Widerlegung muss es in diesem Zusammenhang jedoch fehlen, da die Gesamtzahl der der Untersuchung liegenden Datensätze zu gering ist. Zurückgegriffen werden konnte hier nur auf solche Fragebögen, bei denen zuvor die Mediationsverhandlung gescheitert

534 Zivilgerichte: 9,16\% „eher nein“; 6,25\% „nein“; Sozialgerichte: 15,63\% „eher nein“; $13,54 \%$ „nein“; Familiengerichte: 0,0\% „eher nein“; 5,88\% „nein“; Verwaltungsgerichte: 9,80\% ,eher nein“; $0,0 \%$,nein“. 
war, was insgesamt nur in 73 Fällen vorkam, wobei 21,9\% davon keine Angabe zu der Frage nach einer etwaigen positiven Auswirkung der Mediation auf den weiteren Prozess enthielten.

Von den übrigen Befragten gaben 43,8\% (2,7\% „sehr positiv“; 41,1\% „,eher positiv $^{\text {“) }}$ an, dass die Mediationsverhandlung trotz eines Scheiterns einen positiven Verlauf auf das folgende streitige Verfahren hatte. Dem stehen 34,2\% (2,7\% ,sehr nachteilig“; $31,5 \%$,eher nachteilig $\left.{ }^{6 /}\right)$ der Parteien gegenüber, die die gescheiterte Mediation als nachteilig für den Fortgang des Prozesses ansahen.

Eine Aufschlüsselung in die einzelnen Gerichtszweige soll hier der Vollständigkeit halber zwar aufgeführt werden, eine umfassende Aussagekraft kann hieraus jedoch aufgrund der geringen Anzahl der rückübersandten Fragebögen ${ }^{535}$ nicht gezogen werden.

Bei den Zivilgerichten, die immer noch die größte Gesamtzahl an Fragebögen stellen, waren immerhin 52,63\% (5,26\% „,sehr positiv“; 47,37\% „eher positiv“) der Meinung, eine positive Auswirkung des gescheiterten Mediationsverfahrens auf den Fortgang des Prozesses feststellen zu können; während 31,58 \% (2,63\% „,sehr nachteilig“; 28,95\% ,eher nachteilig“) genau der gegenteiligen Ansicht sind. 15,79\% der Parteien machten keine Angabe hierüber.

Auch bei den Sozialgerichten gab mit 46,67\% der größte Teil der befragten Parteien an, dass die Mediation eher positiv auf den Fortgang der Verhandlung gewirkt hat, währen 20,0\% der gegenteiligen Ansicht waren. Ein Drittel der befragten Parteien machten keine Angaben zu der Frage.

Anders hingegen die Verteilung bei den Familien- und Verwaltungsgerichten. Hier war der größte Teil der Parteien der Ansicht, dass die gescheiterte Mediation negative Auswirkungen auf den Fortgang des Verfahrens hatte. ${ }^{536}$ Während bei den Familiengerichten 16,67\% und bei den Verwaltungsgerichten 28,57\% keine Angabe $\mathrm{zu}$ der entsprechenden Frage machten, gaben bei den Familiengerichten 33,33\% und bei den Verwaltungsgerichten 21,43\% an, dass die Mediation sich positiv auf das weitere Verfahren ausgewirkt habe.

Die Annahme, dass sich sogar eine gescheiterte Mediation auch positiv auf die zukünftigen längerfristigen Parteibeziehungen auswirken kann, lässt sich hingegen durch die empirische Untersuchung nicht bestätigen. Vielmehr gab hier der über-

\footnotetext{
535 Zivilgerichte 38; Sozialgerichte 15; Familiengerichte 6; Verwaltungsgerichte 14.

536 Familiengericht: 50,0\% „,eher nachteilig“; 0,0\% „,sehr nachteilig“; Verwaltungsgericht: 7,14\% „sehr nachteilig“; 42,86\% ,eher nachteilig“.
} 
wiegende Teil der befragten Parteien (5,5\% „sehr nachteilig“; 37,0\% „eher nachteilig") an, dass die gescheiterte Mediation eher nachteilige Auswirkungen auf die zukünftigen Beziehungen gehabt hätte. Während keine Partei die gescheiterte Mediation als sehr positiv für den Fortbestand der Beziehungen empfand, gaben immerhin 32,9\% der Parteien an, dass die Mediation trotz Scheiterns sich eher positiv auf die Beziehung ausgewirkt habe. Bei diesem Ergebnis ist jedoch zu berücksichtigen, dass es insgesamt auf lediglich 73 Fragebögen beruht, von denen mit 24,7\% knapp ein Viertel keine Angabe zu dieser Frage enthielt.

558 Aufgrund des geringen Zahlenmaterials noch weniger aussagekräftig, aber dennoch nicht unerwähnt bleiben soll die Aufschlüsselung in die einzelnen Gerichtsbarkeiten.

559 Von den insgesamt 38 Fragebögen aus dem Bereich der Zivilgerichte enthielten 23,68\% keine Aussage über die Frage nach den künftigen Parteibeziehungen. Darüber hinaus gaben 44,73\% (5,26\% ,sehr nachteilig“; $39,47 \%$,eher nachteilig“) an, dass das Scheitern der Mediation eher nachteilige Folgen für den Fortbestand der Parteibeziehungen gehabt hätte. Lediglich bei 31,58\% schien sich die Mediation trotz Scheiterns positiv auf den weiteren Verlauf der Beziehungen ausgewirkt zu haben.

560 Ähnlich gestaltet sich die Lage im Bereich der Verwaltungsgerichte. Während von den 14 Parteien insgesamt 21,43\% nicht auf die Frage antworteten, gaben 57,15\% $(14,29 \%$,sehr nachteilig“; $42,86 \%$,eher nachteilig“) an, dass das Scheitern der Mediation negative Folgen für die Beziehungen zur anderen Partei gehabt habe. Bei lediglich 21,43\% waren positive Auswirkungen zu spüren.

561 Ähnlich deutlich sehen die Quoten bei den Familiengerichten aus. Hier gaben 50,0\% der Parteien an, dass die Mediation eher nachteilig gewesen sei, während lediglich 33,33\% eine eher positivere Wirkung zu verzeichnen schienen. Hier ist jedoch zu berücksichtigen, dass insgesamt lediglich sechs Parteien Fragebögen ausfüllten, von denen einer - mit anderen Worten 16,67\% - sich nicht zu der Frage äußerten.

562 Einzig bei den Sozialgerichten, bei denen 15 Parteien Fragebögen ausfüllten, von denen ein Drittel keine Angabe zu der betreffenden Frage machten, gab der mit 46,67\% überwiegende Teil an, eher positive Auswirkungen auf die zukünftigen Beziehungen zur anderen Partei zu spüren. 20,0\% hingegen verzeichneten eher nachteilige Auswirkungen. 


\section{Durchsetzbarkeit des Mediationsergebnisses}

\section{a) Mitsprachemöglichkeiten}

Auch wenn festgestellt wurde, dass die Zielsetzung, zu einer einvernehmlichen Lösung zu gelangen, noch kein Garant dafür ist, dass das erzielte Ergebnis von Dauer ist, so lassen sich hieraus dennoch gewisse Rückschlüsse ziehen. Durch die empirische Begleitforschung lässt sich zumindest festhalten, dass eine häufig für die Parteien ausschlaggebende Motivation, an einem Mediationsverfahren teilzunehmen, die der größeren Mitsprachemöglichkeit im Verfahren im Vergleich zu einem streitigen Prozess ist. Durch diese größere Mitsprache- und damit auch Gestaltungsmöglichkeit erhoffen sich viele der Parteien, zu einer einvernehmlichen Lösung zu gelangen, die sie - da sie daran selber konstruktiv mitgewirkt haben - leichter akzeptieren können, als ein „oktroyiertes“ Urteil.

In der Gesamtauswertung sehen 63,9\% zumindest zum Teil (34,7\% ,ja“; 29,2\% „eher ja“) die größere Mitsprache- und Gestaltungsmöglichkeit als Motivation für die Teilnahme an einem Mediationsverfahren an. Demgegenüber war die Mitsprachemöglichkeit für 32,2\% der befragten Parteien eher unwichtig $(17,3 \%)$ bzw. überhaupt nicht relevant (14,9\%). 4,0\% der insgesamt 404 befragten Parteien gaben auf diese Frage keine Auskunft.

Auch eine Aufschlüsselung in die einzelnen Gerichtszweige ergibt, zumindest vom Ergebnis her betrachtet, keine großen Unterschiede. Für den überwiegenden Teil der Befragten, gleichgültig welcher Gerichtsbarkeit ihr jeweiliger Streitgegenstand zugeordnet war, ist die größere Mitsprachemöglichkeit ein Anreiz, an der Mediation teilzunehmen. So gaben bei den zivilgerichtlichen Streitigkeiten 70,83\% (40,0\% „ja“; 30,83\% ,eher ja“) an, dass die größere Mitsprachemöglichkeit für sie zumindest zum Teil ausschlaggebend war. Bei den Sozialgerichten waren es 50,0\% $(22,92 \%$, ja“; $27,08 \%$, ,eher ja“) und bei den Verwaltungsgerichten 49,02\% (19,61\% ,ja“; $29,41 \%$,eher ja“), die dies als Motiv angaben. Berücksichtigt man, dass bei den Sozialgerichten 5,21\% und bei den Verwaltungsgerichten 3,92\% der Parteien keine Angabe zu dieser Frage machten, stellen die jeweiligen Wahrscheinlichkeiten die Mehrheit dar. Im Rahmen der Familiengerichtsbarkeit gaben sogar 88,24\% (70,59\% ,ja“; $17,65 \%$,eher ja“) an, dass die größere Mitsprachemöglichkeit zu den Beweggründen gehört, warum sie an einem Mediationsverfahren teilgenommen haben. Dem stehen bei den Zivilgerichten 25,425\% (8,75\% „nein“; 16,67\% ,eher nein“), bei den Sozialgerichten 44,79\% (25,0\% „nein“; $19,79 \%$,eher nein"), den Familiengerichten 11,76\% (11,76\% „,nein"; 0,0\% „,eher nein") und den Verwaltungsgerichten 47,06\% (25,49\% „nein“; 21,57\% „eher nein“) gegenüber. 
566 Dementsprechend ergibt sich für jeden Gerichtszweig ein Anreiz für die Mediation schon daraus, dass sich der Partei die Möglichkeit eröffnet, den „eigenen Prozess" konstruktiv mitzugestalten und sie nicht dazu verpflichtet ist, passiv all das hinzunehmen, was der Richter ihr vorgibt.

\section{b) Konstruktive Mitarbeit}

567 Diese Motivation der Parteien, sich in die Mediation zu begeben, um an der entstehenden Vereinbarung konstruktiv mitwirken zu können, wurde zum überwiegenden Teil - und auch hier wieder in allen Gerichtsbarkeiten - auch nach dem Mediationsverfahren bestätigt.

568 Als Grundlage für die Auswertung dieser Frage standen 314 Fragebögen von den Parteien zur Verfügung, deren Mediationsverfahren mit einer Vereinbarung endete. Lediglich ein Fragebogen, was ein Quote von 0,3\% entspricht, enthielt keine Angaben zu der gestellten Frage. Von den 313 Parteien, die antworteten, gaben insgesamt 87,6\% (27,7\% ,sehr stark“; 59,9\% „,recht deutlich“) an, dass sie an der Entstehung der Vereinbarung beteiligt waren. Die restlichen 12,1\% hingegen hatten den Eindruck gewonnen, nur wenig (11,5\%) oder gar nicht $(0,6 \%)$ an der Entstehung der Vereinbarung beteiligt zu sein.

569 Ähnlich stellen sich auch die Wahrscheinlichkeiten bei einer Aufschlüsselung nach den einzelnen Gerichtsbarkeiten dar. Im Rahmen der Zivilgerichtsbarkeit, auf die insgesamt 192 Fragebögen entfallen, von denen alle auf die Frage eine Antwort enthielten, geben 85,94\% (19,27\% ,sehr stark“; 66,67\% ,,recht deutlich“) an, an der Findung der Vereinbarung beteiligt zu sein. Dem stehen lediglich 14,06\% der Befragten gegenüber, die sich bei der Findung der Vereinbarung eher übergangen fühlten. Dabei splittet sich diese Angabe in 13,02\% der Parteien auf, die sich zumindest noch ein wenig an der Findung der Vereinbarung beteiligt und 1,04\%, die sich völlig übergangen fühlten.

570 Bei der Sozialgerichtsbarkeit, von der immerhin noch 78 Fragebögen stammen, von denen auch alle eine Antwort auf die Frage nach der Beteiligung an der Vereinbarung enthielten, sind es sogar 94,87\% (50,0\% „sehr stark“; 44,87\% ,recht deutlich“) der Parteien, die ihrer Ansicht nach die Vereinbarung mitgestaltet haben. Lediglich 5,13\% sahen sich während der Mediation in einer eher untergeordneten Position, da sie ihrer Ansicht nach die Vereinbarung lediglich ein wenig zu beeinflussen vermochten.

571 Die Verwaltungsgerichte bewegen sich von den Verhältnissen in etwa in dem Rahmen der Zivilgerichte, auch wenn hier mit 35 Fragebögen, von denen einer keine Angaben zu der gestellten Frage enthielt, eher keine gesicherten statistischen Erkenntnis, sondern allenfalls Tendenzen angenommen werden können. Von den 
befragten Parteien im Bereich des Verwaltungsgerichts gaben 80,0\% (25,71\% „sehr stark“; 54,29\% „,recht deutlich“) an, dass sie die Vereinbarung, welche in der Mediation getroffen wurde, mitgestaltet hätten. Dem stehen lediglich 17,14\% der Parteien gegenüber, die ihre Beteiligung an der Vereinbarung als gering einschätzen. Im Bereich der Verwaltungsgerichte gab es niemanden, der davon ausging, überhaupt nicht an der Vereinbarung beteiligt worden zu sein.

Noch weniger gesicherte Erkenntnisse, als dies im Bereich der Verwaltungsgerichte der Fall ist, können für die Familiengerichte abgeleitet werden. Hier lässt sich die Auswertung lediglich auf neun Fragebögen stützen, von denen aber immerhin alle eine Antwort auf die gestellte Frage enthalten. Nichtsdestotrotz entspricht die Wahrscheinlichkeitsverteilung derjenigen der anderen Gerichtzweige. So geben 88,89\% (22,22\% „sehr stark“; 66,67\% „recht deutlich“) an, an der Entscheidungsfindung beteiligt zu sein. Während sich bei den Familiengerichten niemand überhaupt nicht an der Vereinbarungsfindung beteiligt fühlte, waren es zumindest $11,11 \%$ die sich nur ein wenig beteiligt fühlten.

Daraus ergibt sich, dass die Parteien nicht nur aufgrund der Motivation in die Mediation gehen, die Entscheidung grundlegend selbst mitzubestimmen, sondern dass ihnen dies in den meisten Fällen nach ihrer eigenen Einschätzung auch im Nachhinein in den meisten Fällen gelungen ist. Insoweit lässt sich auch die Annahme aus der ökonomischen Analyse, dass die Parteien sich eher an Vereinbarungen halten, die sie selbst mit beeinflusst haben, stützen, soweit die Parteien mit dem Ergebnis aufgrund der eigenen Einflussnahme zufriedener sind.

\section{c) Beeinflussung durch den Mediator}

Entsprechend der Frage nach der Möglichkeit der Mitgestaltung der Vereinbarung durch die Parteien, fällt die Wahrscheinlichkeitsverteilung im Bezug darauf, ob die Parteien durch den Mediator gedrängt wurden, jener Vereinbarung zuzustimmen, aus. Lediglich 4,7\% (1,0\% ,ja“; $3,7 \%$,eher ja“ $)$ der 311 Parteien, die auf die Frage antworteten - drei machten keine Angabe zu der Fragestellung - fühlten sich vom Mediator zu einer Entscheidung gedrängt. Die übrigen $94,2 \%$ ( $73,2 \%$ „nein“; 21,0\% „eher nein“) sahen den Mediator als neutrale Person an, der sie zu keinen Entscheidungen nötigte.

Damit einher geht auch die Wahrscheinlichkeitsverteilung bezüglich der einzelnen Gerichtszweige. Bei den Zivilgerichten waren es 5,73\% (0,52\% „,ja“; 5,21\% „eher ja“), bei den Sozialgerichten 2,56\% (1,28\% ,ja“; $1,28 \%$,eher ja“), bei den Familiengerichten $11,11 \%(0,0 \%$, ,ja “; $11,11 \%$,eher ja“ $)$ und bei den Verwaltungsgerichten 2,86\% (2,86\% , ,ja“; $0,0 \%$, ,eher ja“), die der Ansicht waren, die in der Mediation getroffene Vereinbarung nicht frei, sondern auf Veranlassung des Mediators 
getroffen zu haben. Der überwiegende Teil der Parteien sah den Mediator jedoch als neutrale Person an, der nicht versuchte, eine bestimmte Vereinbarung herbeizuführen. So gaben in der Zivilgerichtsbarkeit 93,23\% (69,79\% „nein“; 23,44\% „,eher nein“), bei den Sozialgerichten 97,44\% (85,9\% „,nein“; $11,54 \%$,eher nein“), bei den Familiengerichten 77,77\% (44,44\% „,nein“; 33,33\% „eher nein“) und bei den Verwaltungsgerichten 97,14\% (71,43\% „nein“; 25,71\% „eher nein“) der Parteien an, dass der Mediator sie nicht zu einer Vereinbarung gedrängt hätte. Bei diesen Wahrscheinlichkeiten gilt es auch wieder zu berücksichtigen, dass bei der Familiengerichtsbarkeit und bei dem Verwaltungsgericht die angegebenen Prozentzahlen aufgrund der der Untersuchung zugrunde liegenden geringen Anzahl an Fragebögen - bei den Familiengerichten waren dies neun, wovon einer keine Angaben zu der genannten Frage enthielt, bei den Verwaltungsgerichten immerhin 35 - lediglich eine vorsichtige Tendenz widerspiegeln, jedoch kein statistisch gefestigtes Ergebnis darstellen kann. Anders hingegen bei den Zivilgerichten: Hier liegen der Untersuchung insgesamt 192 Fragebögen zugrunde, von denen lediglich zwei keine Angabe enthielten; bei den Sozialgerichten stützen sich die empirischen Untersuchungen auf 78 Fragebögen, die eine Antwort auf die gestellte Frage enthielten.

576 Auch im Zusammenhang mit den Anwälten fühlte sich der überwiegende Teil der Parteien nicht dazu gedrängt, einer Mediationsvereinbarung zustimmen zu müssen. Naturgemäß fällt der Prozentsatz derjenigen, die sich von ihrem Anwalt zu einer Vereinbarung gedrängt sahen höher aus, als dies bei den neutralen Mediatoren der Fall ist, da die Aufgabe der Anwälte eben in einer umfassenden Beratung ihres Mandanten besteht. Damit einher geht auch die Pflicht des Anwalts, seinem Mandanten aufzuzeigen, ob ein Mediationsergebnis, auf das dieser sich gerne einlassen würde, für ihn von Vorteil wäre.

577 So fühlten sich insgesamt im Zusammenhang mit ihrem Anwalt 13,0\% (2,2\% ,ja“; $10,8 \%$,eher ja“) der 314 Parteien, die Fragebögen zurückgegeben hatten, zu einer Vereinbarung gedrängt; bei 79,6\% (63,7\% „,nein“; 15,9\% ,eher nein“) war dies nicht der Fall. 7,3\% der Befragten machten keine Angabe hierzu.

578 Auch hier gibt es wieder keine signifikante Abweichung der einzelnen Gerichtsbarkeiten von der Gesamtauswertung, außer im Bereich der Familiengerichtsbarkeit. Hier gaben 100\% (77,78\% „,nein“; 22,22\% „,eher nein“) der Parteien an, dass ihr Anwalt nicht versucht habe, sie in Richtung eines bestimmten Ergebnisses zu beeinflussen. Dieses außergewöhnliche Ergebnis ist jedoch vor dem Hintergrund der geringen Gesamtzahl der Fragebögen, die im Rahmen dieser Gerichtsbarkeit zur Verfügung stehen - es waren insgesamt nur neun - zu relativieren. Demnach kann hieran zwar eine dahingehende Tendenz gesehen werden, dass in Prozessen der Familiengerichtsbarkeit überwiegend kein Einfluss der Anwälte auf die Ent- 
scheidung der Parteien ausgeübt wird, eine gesicherte statistische Erkenntnis, dass solche Fälle nicht existent sind, kann hieraus jedoch nicht abgeleitet werden.

Bei den Zivilgerichten, denen eine Gesamtsumme von 192 Fragebögen zugrunde liegt, von denen 1,56\% keine Angabe zu der Frage nach der Beeinflussung der Entscheidung der Partei zu einer Vereinbarung durch den Anwalt enthielten, gaben 79,17\% (57,29\% „,nein“; 21,88\% „,eher nein“) an, nicht durch ihren Anwalt zu einer Entscheidung gedrängt worden zu sein. Dies war lediglich bei 19,27\% $(16,67 \%$, eher ja“; $2,60 \%$,jac) der Fall.

Bei den Sozialgerichten liegt die Verteilung ähnlich. Auch hier gab der überwiegende Teil der Parteien (80,77\%, aufgeschlüsselt in 74,36\% „nein“; 6,41\% „eher nein") an, dass keine Beeinflussung durch den Rechtsanwalt stattgefunden habe. Nur 2,56\% gaben an, dass sie definitiv von ihrem Anwalt in Bezug auf eine bestimmte Vereinbarung gedrängt wurden. 16,67\% äußerten sich bei den Sozialgerichten nicht zu einer etwaigen Beeinflussung durch den Anwalt.

Ebenso gestaltet sich das Ergebnis bei den Verwaltungsgerichten. Auch hier gaben 74,29\% (71,43\% „,nein“; 2,86\% „eher nein“) an, beim Treffen der Mediationsvereinbarung nicht durch ihren Anwalt geleitet worden zu sein, was lediglich bei 5,71\% (eher ja) der Fall gewesen ist. 20,0\% machten keine Angabe zu der Frage.

\section{d) Erreichen der selbst gesteckten Ziele}

Ein weiteres wichtiges Kriterium bezieht sich auf das Erreichen der selbst gesteckten Ziele - eng verknüpft insofern mit der Frage, ob die Parteien mit der getroffenen Vereinbarung zufrieden sind und sich dementsprechend eher an sie halten werden, als dies der Fall wäre, hätten sie eine Entscheidung aufoktroyiert bekommen. Legt man für die empirische Untersuchung dieser Annahme die Gesamtauswertung zugrunde, so ergibt sich, dass die Mediation durchaus geeignet ist, den Parteien zu einem Großteil (68,2\% „einiges“) wesentliche Aspekte dessen, was sie durch das Verfahren erreichen wollen, zukommen zulassen und das es in der Mediation sogar bei einem guten Fünftel (20,4\%) gelingt, dass die Parteien eigentlich alle ihr Ziele zu erreichen. Zusammenfassend lässt sich demnach feststellen, dass 88,6\% der Parteien mit dem erreichten Mediationsergebnis durchaus zufrieden sind. Auf der anderen Seite sind es 9,2\% der Parteien, die durch die Mediation kaum etwas $(7,0 \%)$ oder gar überhaupt nichts $(2,2 \%)$ erreicht haben.

Bis auf die Familiengerichtsbarkeit, bei denen die Wahrscheinlichkeitsverteilung etwas von der Gesamtbetrachtung abweicht, wobei dies auch hier durch die geringe Gesamtzahl der Fragebögen - es waren neun, von denen 22,22\% keine Angabe zu der Frage enthielten - bedingt sein kann, fallen die Quoten für die Gerichts- 
zweige für sich betrachtet ähnlich aus. So haben bei den Zivilgerichten 86,98\% (18,75\% „eigentlich alles“; 68,23\% „einiges“) der Parteien einen Großteil dessen erreichen können, was sie sich erhofft hatten. Bei lediglich 11,98\% (3,65\% ,überhaupt nicht“; $8,33 \%$,kaum etwas“) gelang den Parteien das nicht und sie blieben weit hinter dem zurück, was sie sich versprochen hatten. 1,04\% der 192 zurückgesandten Fragebögen enthielten keine Angabe zu der Frage.

584 Noch deutlicher fielen die Auswertungen der Sozial- und Verwaltungsgerichte im Hinblick auf das Erreichen von Zielen in der Mediationsvereinbarung aus. Bei den Sozialgerichten gaben $97,44 \%$ (23,08\% „eigentlich alles“; $74,36 \%$,einiges“) an, größtenteils in den Vereinbarungen das erreicht zu haben, was sie erreichen wollten. Nach den Parteienangaben kam es nicht vor, dass in den sozialgerichtlichen Mediationsfällen niemand kaum etwas oder gar überhaupt nichts von dem in der Mediation erreicht hat, was er hatte erreichen wollen. Die fehlenden 2,56\% ergeben sich daraus, dass zwei der insgesamt 78 Parteien keine Angabe zu dieser Frage machten.

585 Bei den Verwaltungsgerichten war es eine Partei von insgesamt 35, die sich nicht zu der gestellten Frage äußerte, was einer Quote von 2,86\% entspricht. Daneben konnten 85,72\% (22,86\% ,eigentlich alles“; 62,86\% „einiges“) der Befragten ihre Vorstellungen verwirklichen. 11,43\% hingegen konnten von dem, was sie gerne erreicht hätten, kaum etwas verwirklichen.

\section{e) Gerechtigkeit der Vereinbarung}

586 Ein weiteres wichtiges Indiz für die Zufriedenheit der Parteien mit der Mediationsvereinbarung ist das Empfinden der Parteien im Hinblick auf die Gerechtigkeit der getroffenen Vereinbarung. Von den insgesamt 314 zurückgesandten Fragebögen, von denen 2,2\% keine Angabe zu der Frage nach der Gerechtigkeit der Vereinbarung enthielten, gaben 76,1\% (29,0\% ,ja“; 47,1\% „,eher ja“) an, dass sie die getroffene Vereinbarung für gerecht halten. Dem stehen 21,6\% (5,7\% „nein“; 15,9\% „eher nein“) gegenüber, denen das Mediationsergebnis ungerecht erscheint.

587 Ähnliche Ergebnisse ergeben sich auch in den einzelnen Gerichtszweigen. Lediglich die Familiengerichte fallen hier etwas aus dem Rahmen, was jedoch dadurch bedingt sein mag, dass die zugrunde liegende Gesamtzahl der Fragebögen mit neun zu niedrig ist, um hieraus genaue statistische Aussagen ableiten zu können. Erschwerend kommt hinzu, dass zwei dieser Fragebögen, was schon einer Quote von 22,22\% entspricht, sich nicht zu der gegebenen Frage äußerten. Der Vollständigkeit halber sei hier das Ergebnis der Umfrage jedoch dennoch dargestellt: Während 44,44\% (22,22\% ,ja“; $22,22 \%$,eher ja“) der Parteien der Ansicht waren, dass das in der Mediation erreichte Ergebnis gerecht war, gaben doch immerhin 
33,33\% (0,0\% „nein“; 33,33\% „eher nein“) an, dass dies ihrem Empfinden nach nicht der Fall gewesen sei.

Bei den Gerichtsbarkeiten, bei denen eine größere auswertbare Gesamtsumme an Fragebögen gegeben ist, decken sich die Ergebnisse weitestgehend mit der Gesamtauswertung. So gaben von den insgesamt 192 von den Zivilgerichten eingereichten Fragebögen 71,35\% (25,0\% ,ja“; 46,35\% „,eher ja“) der Parteien an, das Ergebnis der Mediationsverhandlung für gerecht $\mathrm{zu}$ halten, während 26,56\% $(8,33 \%$ „nein“; 18,23\% „,eher nein“) dies für ungerecht hielten. Bei 2,08\% der Fragebögen fehlte eine entsprechende Angabe.

Vergleichbar gestalten sich die Wahrscheinlichkeitsverteilungen am Verwaltungsgericht. Hier waren es 77,14\% (25,71\% ,ja“; 51,43\% „eher ja“) der 35 Parteien, die auf diese Frage eine Antwort gaben, die das erzielte Ergebnis für gerecht hielten. Dem steht eine Quote von 20,0\% (5,71\% „,nein“; 14,29\% „,eher nein“) der Parteien gegenüber, die die erzielte Vereinbarung als ungerecht ansahen. 2,86\% machten hierzu keinerlei Angaben.

Das deutlichste Ergebnis wurde im Bereich der Sozialgerichtsbarkeit erzielt. Von den 78 befragten Parteien gaben alle eine Antwort auf die Frage nach der Gerechtigkeitsempfindung im Zusammenhang mit dem Mediationsergebnis. Von diesen gaben 91,3\% (41,03\% ,ja“; 50,0\% „,eher ja“) an, dass sie die Mediationsvereinbarung für gerecht erachten, während sich lediglich 8,97\% (0,0\% „,nein“; 8,97\% „eher nein“) der Befragten dahingehend äußerten, dass dem nicht so sei.

\section{f) Zufriedenheit mit dem Ergebnis insgesamt}

Mit den Fragen nach der Gerechtigkeit und der Möglichkeit des eigenen Mitgestaltens der Mediationsvereinbarung einher geht auch die Frage nach der Zufriedenheit mit dem Ergebnis insgesamt. Dementsprechend ähnlich fällt auch das Ergebnis der Frage nach der Gesamtzufriedenheit im Vergleich zu den anderen Fragen aus. So gaben von den 314 befragten Parteien, von denen lediglich 1,6\% keine Angabe zu der Frage gemacht hatten, 85,6\% (24,8\% „sehr zufrieden“; 60,8\% „eher zufrieden") an, mit dem Ergebnis der Mediation insgesamt zufrieden zu sein. Lediglich 12,8\% (2,9\% ,sehr unzufrieden“; 9,9\% „,eher unzufrieden“) gaben an, mit dem Mediationsergebnis insgesamt nicht zufrieden zu sein.

Auch hier ergeben sich bei einer Aufschlüsselung in die einzelnen Gerichtszweige, mit Ausnahme der Familiengerichtsbarkeit - was durch die geringe Anzahl der beantworteten Fragebögen, es waren hier nur neun Stück von denen jedoch alle eine Antwort auf die Frage nach der Gesamtzufriedenheit enthielten, bedingt ist keine signifikanten Abweichungen von der Gesamtauswertung. 66,66\% (22,22\% „sehr zufrieden“; 44,44\% „,eher zufrieden“) der befragten Parteien gaben hier an, 
mit dem Mediationsergebnis zufrieden zu sein, das restliche Drittel $(11,11 \%$,sehr unzufrieden“; 22,22\% „eher unzufrieden“) war mit dem erzielten Ergebnis nicht einverstanden.

593 Bei den Zivilgerichten waren es 14,07\% (3,13\% „,sehr unzufrieden“; 10,94\% „,eher unzufrieden“), die mit dem gesamten Mediationsergebnis unzufrieden waren. Dem stehen 84,9\% (23,44\% „,sehr zufrieden“; 61,46\% „,eher zufrieden“) der Parteien gegenüber, die das Ergebnis als positiven Gesamtabschluss betrachteten. Von den 192 Fragebogen der Zivilgerichtsbarkeit enthielten lediglich zwei, was einer Quote von 1,04\% entspricht, keine Angabe über die Gesamtzufriedenheit.

594 Eine ähnliche Wahrscheinlichkeitsverteilung wie bei den Zivilgerichtsbarkeiten ergibt sich im Bereich der Verwaltungsgerichtsbarkeit. Auch hier ist der Großteil, genauer 76,97\% (22,86\% ,sehr zufrieden“; 54,29\% „,eher zufrieden“) der 35 Parteien, die Fragebögen zurücksandten, mit dem Gesamtergebnis der Mediation zufrieden. Während eine Partei, sprich 2,86\% nicht zu der Frage äußerten, gaben 20,0\% (2,86\% „sehr unzufrieden“; $17,14 \%$,eher unzufrieden“) an, mit der getroffenen Mediationsvereinbarung unzufrieden zu sein.

595 Im Bereich der Sozialgerichtsbarkeit liegt die Zufriedenheit der Parteien noch deutlich über dem Durchschnitt der Gesamtauswertung. Hier gaben 93,59\% (29,49\% „sehr zufrieden“; 64,1\% „eher zufrieden“) der insgesamt 78 im Bereich der Sozialgerichtsbarkeit befragten Parteien an, mit dem Ergebnis zufrieden zu sein, während lediglich 3,84\% (1,28\% „sehr unzufrieden“; $2,56 \%$ „eher unzufrieden $\left.^{\prime \prime}\right)$ hiermit unzufrieden waren. Zwei Parteien und damit eine Quote von 2,56\% äußerten sich nicht zu der Gesamtzufriedenheit.

\section{g) Dauerhafte Lösung des Konflikts}

596 Im Rahmen der ökonomischen Analyse wurde die These aufgestellt, dass die Parteien zwar eher bereit sind, sich an eine Vereinbarung zu halten, wenn sie selbst an ihrer Findung aktiv beteiligt waren, dass dies jedoch noch kein Garant dafür sei, dass diese getroffene Vereinbarung gegen einen Bruch immun ist, sodass es hier unbedingt einer Absicherung der Durchsetzbarkeit der Mediationsvereinbarung bedürfe.

597 Im Rahmen der empirischen Begleitforschung wurden die Parteien, nachdem die Mediationsverhandlung mit einer Vereinbarung endete, gefragt, inwieweit sie der Ansicht seien, dass der Konflikt nun dauerhaft gelöst sei. Sowohl der überwiegende Teil der befragten Parteien in der Gesamtauswertung als auch in den einzelnen Gerichtszweigen gab hier an, dass der Konflikt, um den es gegangen war, nun tatsächlich dauerhaft beigelegt worden sei. Von den 314 Parteien, die in der Statistik für die Gesamtauswertung erfasst sind, gaben dies 78,7\% (39,5\% ,,ja“; 39,2\% 
„eher ja“) an. Während 1,3\% sich gar nicht zu der Frage äußerte, waren 20,2\% (7,0\% „,nein“; $13,2 \%$,eher nein“) der befragten Parteien der Meinung, dass der Konflikt durch die Mediation noch nicht dauerhaft gelöst worden sei.

Ähnlich gestaltet sich die Statistik im Bereich der Zivilgerichte. Von den insgesamt 192 befragten Parteien gaben 80,21\% (41,67\% ,ja“; 38,54\% ,eher ja“) an, dass ihr Konflikt durch die Mediation zu einem dauerhaften Abschluss gekommen sei, wohingegen 18,23\% (6,77\% „nein“; $11,46 \%$, „eher nein“) angaben, dass dies nicht der Fall gewesen sei. 1,56\% der befragten Parteien äußerten sich nicht zu diesem Punkt.

Bei den Sozialgerichten stellt sich die Lage ähnlich wie bei den Zivilgerichten dar. Von den 78 befragten Parteien sahen 83,16\% (42,31\% ,ja“; 41,03\% „eher ja“) ihren Konflikt als dauerhaft beigelegt an, während 16,67\% (5,13\% „nein“; $11,54 \%$ „eher nein") angaben, dass der Konflikt mit der anderen Partei auch nach der Durchführung der Mediation immer noch fortbestehe. Alle befragten Parteien machten Angaben zu der gestellten Frage.

Etwas weniger positiv fiel die Auswertung bei den Verwaltungsgerichten aus, obwohl auch hier immer noch gut über die Hälfte, genauer 62,85\% (25,71\% „,ja“; $37,14 \%$,eher ja") der Parteien nach Abschluss der Mediation von einer dauerhaften Konfliktlösung ausging. Dem stehen 34,28\% (8,57\% „nein“; $25,71 \%$ „,eher nein") der Parteien gegenüber, die ihren Konflikt noch nicht als beigelegt betrachten. 2,86\% der Parteien äußerten sich nicht zu der Frage nach einer dauerhaften Konfliktbeilegung.

Bei der Familiengerichtsbarkeit lassen sich auch bei der Frage nach einer dauerhaften Konfliktlösung wieder nur Tendenzen ablesen, da die Gesamtzahl der von den Parteien zurückgesandten Fragebögen mit neun zu gering ist, als dass hieraus statistisch gesicherte Erkenntnisse abgeleitet werden könnten. Jedoch scheint es auch hier so zu sein, dass der überwiegende Teil, nämlich zwei Drittel $(22,22 \%$,ja“; $44,44 \%$,eher ja“) der Parteien von einer dauerhaften Konfliktlösung ausgehen, während das übrige Drittel (22,22\% „nein“; $11,11 \%$ „eher nein“) annimmt, dass der Konflikt auch weiterhin Bestand hat. Alle neun Parteien äußerten sich zu der gestellten Frage.

\section{h) Vereinbarung eingehalten}

Dass die Befürchtung, wonach sich die Parteien ohne ausreichende Durchsetzungsmöglichkeiten nicht an die in der Mediation getroffene Vereinbarung halten, nicht ganz unbegründet ist, zeigt sich auch im Rahmen der statistischen Auswertung der Nachbefragungsbögen der Parteien. Stellte man alleine auf die Nachbefragungsbögen der Parteien ab, die gefragt wurden, ob sie sich selbst an die 
Mediationsvereinbarung gehalten haben, so erscheint eine ausreichende Durchsetzungsmöglichkeit zunächst noch verzichtbar. Zieht man jedoch auch die Auswertung der Frage, ob die andere Partei die in der Mediation getroffene Vereinbarung eingehalten habe, heran, ergibt sich ein hiervon abweichendes Bild.

603 Bei der Frage, ob sich die befragte Partei selbst an die Vereinbarung gehalten hätte, gaben so gut wie alle an, dass dem so gewesen ist. Lediglich eine $(0,6 \%)$ von 163 befragten Parteien gab zu, sich nicht an die in der Mediation getroffene Vereinbarung gehalten zu haben. Während 2,5\% der Parteien keine Aussage zu der Frage trafen, gaben die übrigen $96,9 \%$ jegliche Vereinbarung eingehalten zu haben.

604 Die Partei, die die Vereinbarung gebrochen hat, stammt aus der Zivilgerichtsbarkeit und stellt hier 1,08\% der insgesamt 93 Befragten dar. 2,15\% machten keine Angaben, während 96,77\% angaben, sich an die Absprachen gehalten zu haben.

605 Von den 29 Parteien aus dem Bereich der Sozialgerichtsbarkeit, die den Fragebogen ausfüllten, enthielten sich 6,9\% jeglicher Antwort, die übrigen 93,1\% gaben an, die Vereinbarungen eingehalten zu haben.

606 Bei den Verwaltungsgerichten gaben alle 41 befragten Parteien an, die Mediationsvereinbarung nicht gebrochen zu haben.

607 Bei der Frage, ob die andere an der Mediation beteiligte Partei die Mediationsvereinbarung eingehalten habe, gab bei der Nachbefragung zwar auch der größte Teil der Parteien $(81,6 \%)$ an, dass dem so sei, in 11,7\% der Fälle hingegen wurde die Mediationsvereinbarung von der anderen Partei jedoch gebrochen; 6,7\% der 163 Befragten äußerten sich nicht auf die Frage nach einem Bruch der getroffenen Vereinbarung. Da dies immerhin über ein Zehntel ist, erscheint es, wie in der ökonomischen Analyse bereits angeklungen, durchaus empfehlenswert, die Mediationsentscheidungen auch trotz des der eigenen Gestaltung durch die Parteien und des hohen Zufriedenheitsgrades mit dem erzielten Ergebnis durch etwaige Durchsetzungsmaßnahmen abzusichern.

608 Gliedert man dieses Gesamtergebnis darüber hinaus noch in die einzelnen Gerichtsbarkeiten auf, so empfiehlt es sich insbesondere für die Zivil- und die Verwaltungsgerichte die Mediationsergebnisse durch Durchsetzungsmaßnahmen zu stützen. In diesen beiden Gerichtszweigen lagen die Quoten des Nichteinhaltens der getroffenen Vereinbarung bei über 12\% (Zivilgericht 12,9\%; Verwaltungsgericht 12,2\%). Lediglich am Sozialgericht lag diese Quote mit 6,9\% deutlich niedriger als der Durchschnitt. Dem stehen Wahrscheinlichkeiten von 80,65\% für die Zivilgerichte, $89,66 \%$ für die Sozialgerichte und 78,05\% für die Verwaltungsgerichte gegenüber, in denen sich die andere Partei an die getroffene Vereinbarung 
gehalten hat. Keine Angaben machten bei den Zivilgerichten 6,45\% der 93 Parteien, bei den Sozialgerichten 3,45\% der 29 Parteien und bei den Verwaltungsgerichten $9,76 \%$ der 41 Befragten.

\section{Zufriedenheit mit dem Verfahren und Ergebnis der Mediation}

Ein weiterer wesentlicher Vorteil des Mediationsverfahrens im Vergleich zum streitigen Verfahren ist nach ökonomischer Analyse des Rechts, dass die Verfahrensbeteiligten mit dem Mediationsergebnis in der Regel zufriedener sind, als dies bei einem streitigen Gerichtsverfahren der Fall ist.

\section{a) Mediationsverfahren}

In diesem Zusammenhang stellt schon die Flexibilität des Mediationsverfahrens einen wesentlichen Vorteil der Mediation gegenüber herkömmlichen Gerichtsverfahren dar, da die Parteien die Möglichkeit haben, mit Hilfe des Mediators das Verhandlungsprocedere ihren Bedürfnissen entsprechend selbst zu gestalten.

Dass ein wesentlicher Vorteil der Mediation darin besteht, dass die Parteien konstruktiv an dem Ergebnis mitwirken, wurde bereits im Zusammenhang mit der Durchsetzbarkeit des Mediationsergebnisses bei Untersuchung der Frage, ob diese Gestaltungsmöglichkeit ein Anreiz für die Parteien darstellt, den Weg in die Mediation statt des streitigen Gerichtsverfahrens zu wählen, festgestellt. ${ }^{537}$

Über die Beweggründe für den Weg in die Mediation hinaus wurden die Parteien jedoch auch darüber befragt, ob sie den Gang der Mediation wirklich nach ihren eigenen Vorstellungen mitgestalten konnten oder ob Entscheidungen über ihren Kopf hinweg getroffen wurden. Nach der statistischen Auswertung lässt sich feststellen, dass die Beweggründe vieler Parteien, nämlich die Möglichkeit der aktiven Einwirkung auf die Ergebnisfindung, durch die Mediation im Wesentlichen nicht enttäuscht wurden. So konnten nach der Gesamtauswertung 86,6\% (46,3\% ,ja“; 40,3\% „eher ja“") der Parteien die Mediation nach ihren eigenen Vorstellungen mitgestalten. Lediglich 11,6\% (3,2\% „nein“; $8,4 \%$,eher nein“) war dies nach eigenen Angaben nicht möglich. 1,7\% der Parteien äußerten sich nicht auf diese Frage. Dieses Ergebnis deckt sich auch in etwa mit dem der Frage, ob die Entscheidungen über den Kopf der Parteien hinweg getroffen wurden. Hier gaben 91,4\% (73,8\% „,nein“; $17,6 \%$ „eher nein“) in der Gesamtbetrachtung an, dass die Entscheidungen nicht über ihren Kopf hinweg getroffen wurden. Allerdings fühl-

${ }^{537}$ Siehe hierzu unter Rn. 563. 
ten sich auch 6,2\% (4,5\% „,eher ja“; 1,7\% ,ja“) bei der Entscheidungsfindung übergangen. Die übrigen 2,5\% der Parteien äußerten sich nicht zu dieser Frage.

613 Diese Gesamtergebnisse korrelieren mit den Einzelergebnissen bei den unterschiedlichen Gerichtszweigen. In jeder der vier untersuchten Gerichtsbarkeiten gab der Großteil der befragten Parteien an, dass sie den Ablauf des Mediationsverfahrens aktiv mitgestalten konnten. Bei den Zivilgerichten waren dies 85,42\% (40,42\% ,ja“; 45,0\% „eher ja“), bei den Sozialgerichten 93,75\% (63,54\% ,ja“; 30,21\% ,eher ja“), bei den Familiengerichten 76,47\% (23,53\% ,ja“; 52,94\% ,eher $\mathrm{ja}^{\text {“") }}$ und bei den Verwaltungsgerichten schließlich 82,35\% (49,02\% ,ja“; 33,33\% „eher $\mathrm{ja}^{6}$ ). Dementsprechend verhalten sich die Wahrscheinlichkeitsverteilungen bei den Gerichtsbarkeiten im Hinblick auf die Frage, ob Entscheidungen über den Kopf der Parteien hinweg entschieden wurden. So gaben bei den Zivilgerichten 90,0\% (71,67\% „,nein“; 18,33\% „eher nein“) an, dass nicht über ihren Kopf hinweg entschieden wurde. Bei den Sozialgerichten waren es sogar 96,88 (84,38\% „,nein“; 12,5\% ,eher nein“), bei den Familiengerichten 70,59\% (47,06\% „,nein“; 23,53\% „eher nein“) und bei den Verwaltungsgerichten schließlich 91,5\% (73,75\% „,nein“; $17,75 \%$,eher nein“).

614 Durch die statistische Erhebung lässt sich daher die Annahme aus der ökonomischen Analyse, dass die Mediation gegenüber dem streitigen Verfahren den Vorteil hat, dass die Parteien das Verfahren selber aktiv mitgestalten können, belegen.

615 Der Vollständigkeit halber seien hier noch die Einzelergebnisse im Hinblick darauf aufgeführt, dass die Parteien der Ansicht waren, die Mediation nicht nach eigener Vorstellung mitgestalten zu können bzw. bei der Entscheidungsfindung übergangen wurden. Bei den Zivilgerichten waren es 13,34\% (4,17\% „nein“; 9,17\% „eher nein“) der Parteien, die der Ansicht waren, das Mediationsergebnis nicht nach ihren eigenen Vorstellungen mitgestalten zu können. Während dies bei den Sozialgerichten lediglich 3,12\% (1,04\% „,nein“; 2,08\% „eher nein“) der Befragten so sahen, waren es bei den Familiengerichten immerhin 23,53\% (5,88\% „,nein“; 17,65\% „eher nein“). Die Quote bei den Verwaltungsgerichten beträgt 15,69\% (1,96\% „,nein“; 13,73\% ,eher nein“). Sich nicht zu dieser Frage geäußert haben sich bei den Zivilgerichten 1,25\%, bei den Sozialgerichten 3,13\% und bei den Verwaltungsgerichten 1,96\%. Im Rahmen der Familiengerichtsbarkeit haben alle befragten Parteien auf diese Frage geantwortet.

616 Der Ansicht, dass eine Entscheidung trotz eines Mediationsverfahrens über ihren Kopf hinweg getroffen wurde, waren bei den Zivilgerichten 7,08\% (2,08\% , ,ja“; 5,0\% ,eher ja“), bei den Sozialgerichten 1,04\% (1,04\% ,ja“; 0,0\% ,eher ja“), bei den Familiengerichten 29,41\% (5,88\% ,ja“; 23,53\% „eher ja“) sowie bei den Verwaltungsgerichten 3,92\% (0,0\% ,ja“; 3,92\% „,eher ja“). Keine Angaben zu dieser 
Frage machten bei den Zivilgerichten 2,92\%, den Sozialgerichten 2,08\% und den Verwaltungsgerichten 1,96\%. In der Familiengerichtsbarkeit antworteten alle befragten Parteien auf diese Frage.

Auch dadurch, dass die Parteien das Verfahren der Mediation als fairer und persönlicher als das herkömmliche Gerichtsverfahren erleben, wie schon dargelegt wurde, ${ }^{538}$ steigt ihre Zufriedenheit mit dem Verfahren.

\section{b) Mediationsergebnis}

Wie schon die Auswertungen im Zusammenhang mit der Durchsetzbarkeit des Mediationsergebnisses festgestellte, ist die Zufriedenheit nicht nur mit dem Mediationsverfahren, sondern auch mit dem Mediationsergebnis selbst höher als dies bei einem Gang in das streitige Verfahren der Fall gewesen wäre. ${ }^{539}$ Hieraus lässt sich ableiten, dass die Annahme aus der ökonomischen Analyse, dass die Parteien mit einem Mediationsergebnis zufriedener sind, als dies bei einem Urteil der Fall wäre.

Wie die Auswertung gezeigt hat, bestätigen sich die Bedenken, dass ein etwaiges zwischen den Parteien bestehendes Machtungleichgewicht nachteilige Auswirkungen auf die in der Mediation getroffene Abschlussvereinbarung hat, nicht. Zum einen waren die Parteien nur zu einem geringen Prozentsatz der Überzeugung, vom Mediator zu einer Zustimmung zu einer Vereinbarung gedrängt worden zu $\operatorname{sein}^{540}$ und zum anderen hielt der überwiegende Teil der Parteien die Vereinbarung für gerecht ${ }^{541}$.

Selbst bei der Nachbefragung Parteien bestätigt sich die Befürchtung, dass ein Mediator voreingenommen oder parteiisch war, zum überwiegenden Teil nicht. So gaben 90,8\% (80,4\% „nein“; $10,4 \%$ „eher nein“) der insgesamt 163 befragten Parteien an, dass der Mediator nicht parteiisch oder voreingenommen, sondern vielmehr neutral gewesen sei. Dem stehen lediglich 7,4\% (3,7\% ,ja“; $3,7 \%$, ,eher ja") gegenüber, die diesen Eindruck gewonnen hatten. Fünf Parteien machten keine Angabe zu einer möglichen Voreingenommenheit.

Die Aufschlüsselung in die einzelnen Gerichtszweige ergibt ein ähnliches Bild. Im Bereich der Zivilgerichtsbarkeit waren es 87,1\% (73,12\% „nein“; 13,98\% „eher nein") der befragten Parteien, die den Mediator als neutral empfanden. Während 11,83\% (6,45\% „,ja“; 5,38\% „eher ja“) der insgesamt 93 befragten Parteien anga-

\footnotetext{
538 Siehe hierzu Rn. 563.

539 Siehe hierzu Rn. 563.

540 Siehe hierzu Rn. 563.

541 Siehe hierzu Rn. 563.
} 
ben, den Mediator als voreingenommen empfunden zu haben, enthielten sich $1,08 \%$ einer Aussage.

622 Bei den Verwaltungsgerichten gaben von den 41 Befragten 90,8\% (80,37\% „nein"; $10,43 \%$,eher nein“) an, dass der Mediator nicht parteiisch gewesen sein. Lediglich 7,36\% (3,68\% ,ja“; 3,68\% „eher ja“) waren der Ansicht, dass er eher voreingenommen war. Weitere $1,84 \%$ machten hierzu keine Angabe.

623 Noch deutlicher fallen die Quoten am Sozialgericht aus. Von den 29 befragten Parteien enthielten sich lediglich 3,45\%. Die übrigen $96,55 \%$ ( $91,1 \%$ „nein“; $3,45 \%$,eher nein") gaben an, den Mediator als unparteiisch und unvoreingenommen erlebt zu haben.

\section{Fazit}

624 Nach der empirischen Auswertung empfiehlt es sich, die gerichtsinterne Mediation als dauerhaftes Angebot in die niedersächsische Justiz aufzunehmen.

625 Ein solches empfiehlt sich alleine schon aufgrund der für die Parteien aus der Mediation sich ergebenden Vorteile. Durch die gerichtsinterne Mediation können zum einen das Vertrauen und das Verständnis der Bürger in und für die Justiz und zum anderen auch die Zufriedenheit der betroffenen Parteien mit den erzielten Ergebnissen gesteigert bzw. erreicht werden, was sich nachhaltig auf den Rechtsfrieden zwischen den streitenden Parteien auswirkt. Wie schon im Rahmen der ökonomischen Analyse des Rechts angenommen und wie sich durch die empirische Untersuchung bestätigen ließ, trägt die Mediation zu diesen Gesichtspunkten wesentlich bei.

626 Auch im Hinblick auf die Richterschaft empfiehlt sich eine dauerhafte Einführung der gerichtsinternen Mediation. Wie die empirische Auswertung gezeigt hat, ergeben sich zwar im Bereich der ,gewöhnlichen“ Richter durch eine solche Einführung keine großen Änderungen, doch sind bei den Richtermediatoren durchaus positive Einflüsse der Mediation auf ihren beruflichen Alltag zu spüren. Zum einen werden Fälle in der Mediation schneller abgearbeitet, was auch für das Land selbst eine Kostenersparnis bedeutet, zum anderen wird die Zufriedenheit der betroffenen Richter durch ihre Tätigkeit als Mediator gesteigert, was wiederum eine höhere Belastbarkeit und Produktivität mit sich bringt.

627 Auch aus Rücksicht auf die Anwaltschaft braucht von einer niedersachsenweiten Einführung einer gerichtsnahen Mediation kein Abstand genommen werden, da 
den Anwälten durch diese kein Schaden droht. Für den Rechtsanwalt ergeben sich zwar keine direkten Vorteile aus der Mediation - außer, dass sein Mandant mit dem in der Mediation erzielten Ergebnis zufriedener ist, als dies bei einem Urteil der Fall wäre, wodurch die Reputation des Anwalts steigt - doch braucht die Anwaltschaft auch keine negativen Folgen zu fürchten. Die Hauptbedenken, nämlich ein etwaiger Einnahmeverlust, ließen sich im Rahmen der empirischen Untersuchung nicht bestätigen, vielmehr stehen die Rechtsanwälte nach der Durchführung einer Mediation genauso gut als hätten sie ein Verfahren im Wege des streitigen Prozesses geführt hätten. 


\section{F. Vierter Teil: Zusammenfassung und Ausblick}

628 Das Angebot der gerichtsnahen Mediation hat sich während der Projektlaufzeit als erfolgreiche und auch für die Justiz kostengünstige Alternative zum streitigen Verfahren erwiesen. Für eine dauerhafte Aufnahme der - rechtlich zulässigen gerichtsnahen Mediation in das Leistungsangebot der Justiz besteht jedoch noch erheblicher Handlungsbedarf.

629 Dringend ist die gesetzliche Verankerung des gerichtsinternen Mediationsangebotes aufgrund wettbewerbsrechtlicher Bedenken eines kostenfreien Mediationsangebotes unter den derzeitigen gesetzlichen Rahmenbedingungen zu empfehlen. Eine angemessene Lösung wäre hier die explizite Nennung der gerichtsinternen Mediation im Rahmen des $\int 278$ ZPO sowie in der VwGO und dem SGG, indem die Möglichkeit der Verweisung eines Falles an den Richtermediator zur gerichtsinternen Mediation entsprechend des bisherigen \278 Abs. 5 ZPO formuliert wird. Aufgrund der positiven Erfahrungen im Modellprojekt sollte die Ergänzung auch die Klarstellung enthalten, dass der Richtermediator als durch den gesetzlich zuständigen Richter ersuchten Richter das gerichtliche Verfahren nach Abschluss der Mediation wieder aufrufen und einen von den Parteien in der Mediation geschlossenen Vergleich zu Protokoll nehmen kann. Gleichzeitig sollte die bisher in $\int 278$ Abs. 5 Satz 2 und 3 ZPO enthaltene Möglichkeit des Vorschlags einer auBergerichtlichen Streitschlichtung beibehalten werden, um letztlich den Parteien Alternativen zur Konfliktlösung zur Wahl zu stellen und so auch Wettbewerb zwischen gerichtlichen und außergerichtlichen Konfliktlösungsangeboten herzustellen. Wird von einer solchen Gesetzesänderung abgesehen, so erscheint die Einführung einer angemessenen Kostenregelung unerlässlich.

630 Darüber hinaus sollte zur Klarstellung der dienstrechtlichen Zulässigkeit der Richtermediatorentätigkeit eine (landesrechtliche) Regelung im Sinne von $\int 4$ Abs. 2 Nr. 2 DRiG geschaffen werden, welche dem Richter die Tätigkeit im Rahmen der gerichtsnahen Mediation als ausdrücklich erlaubte Tätigkeit zuweist.

631 Die rechtlichen Beziehungen zwischen den Mediationsparteien und der Justiz können umfassend durch privatrechtliche Vereinbarungen geregelt werden. Um aber die Einheitlichkeit des gerichtlichen Mediationsangebotes an den einzelnen Gerichten sicherzustellen, ist hinsichtlich der Regelung der Pflichten des Mediators die Einbeziehung eines anerkannten Regelwerkes, wie z.B. des europäischen Verhaltenskodex für Mediatoren in die privatrechtlichen Vereinbarungen, zu empfehlen. Eine freiwillige Selbstbindung an einen solchen Verhaltenskodex oder auch eine Zertifizierung der Richtermediatoren kann ein positives Signal zur Ge- 
währleistung bestimmter Qualitätsstandards auf dem Markt für Konfliktlösungsdienstleistungen setzen. Zur langfristigen Qualitätssicherung der Richtermediatorentätigkeit sind darüber hinaus auch Überprüfungen, Supervisionen und regelmäßige Weiterbildungen einzuführen.

Um die Alternative der gerichtsinternen Mediation innerhalb der Justiz nutzbar zu machen und ihren Erfolg langfristig zu sichern, sollte die Fallabgabebereitschaft der gesetzlichen Richter gestärkt bzw. unterstützt werden: Insbesondere sollte die Ausgestaltung des Pensenschlüssels dem Gedanken der Anreizsetzung zur Abgabe von Fällen in die gerichtsnahe Mediation Rechnung tragen. Darüber hinaus wäre auch an die generelle Einführung von Informationsgesprächen über das Angebot der gerichtsinternen Mediation zu denken, was die Akzeptanz des Mediationsangebotes auch in der Richterschaft stärken würde.

Ebenso ist für die Richtermediatoren die Einführung einer einem Deputatsschlüssel vergleichbaren Verpflichtung zur Durchführung einer bestimmten Anzahl von Mediationen zu empfehlen. Als alternativer, die Richtermediatoren stärker motivierender Lösungsansatz kommt hier aber auch eine freiwillige Selbstbindung der Richtermediatoren bei gleichzeitiger mit den Parteien vereinbarten Beschränkung des zeitlichen Rahmens der Mediationsverhandlungen in Betracht. Das in Göttingen erprobte Modell erscheint dabei unter Anreizaspekten als empfehlenswerte Ausgestaltung des Zusammenspiels von Deputatsschlüssel der abgebenden Kammer und freiwilliger Selbstbindung der Richtermediatoren.

Die Einführung des neuen Rechtsanwaltsvergütungsgesetzes (RVG) hat die noch durch die BRAGO bedingten negativen finanziellen Anreize für den Anwalt durch die Empfehlung der gerichtsnahen Mediation deutlich entschärft. Durch die Empfehlung der gerichtsnahen Mediation an seinen Mandanten entsteht nun für den Anwalt kein Gebührenverlust gegenüber dem streitigen Verfahren - bei gleichzeitiger Chance auf eine geringere Arbeitsbelastung durch die Wahl der Alternative der gerichtsnahen Mediation. Ob darüber hinaus zur Förderung der gerichtsnahen Mediation aber auch noch deutliche positive Anreize durch die Gebührenordnung für den Anwalt gesetzt werden sollten, kann derzeit noch nicht beantwortet werden. Allerdings ist bereits jetzt in diesem Zusammenhang zu bedenken, dass dann ein für die Anwaltschaft gesetzter zusätzlicher positiver Anreiz für die Mediation gegebenenfalls durch einen negativen Anreiz für die Konfliktparteien aufgrund höherer Gebühren ausgeglichen werden könnte.

Zur Förderung der gerichtsnahen Mediation, aber auch sonstiger alternativer Streitschlichtungsverfahren, ist darüber hinaus auch die Unterstützung der Justiz zur Einführung einer berufsständischen Verhaltenspflicht für Anwälte zu empfehlen, ihre Parteien umfassend und qualifiziert über alternative Konfliktlösungsan- 
gebote und damit auch die gerichtsnahe Mediation zu informieren. Als Alternative erscheint hier eine Selbstregulierung durch Gütesiegel, Zertifizierung oder Akkreditierung als wirkungsvolles Instrument.

636 Für die Parteien werden die Anreize für die gerichtsnahe Mediation maßgeblichen durch die Kostenrisiken bestimmt, weshalb bei einer langfristigen Aufnahme der Mediation in das Leistungsangebot der Justiz auf die Ausgestaltung der Kostenstrukturen besonderes Augenmerk gelegt werden muss, um negative Anreize zu vermeiden. Werden langfristig für die gerichtsnahe Mediation Gebühren eingeführt, so sollten diese deutlich unter den im Rahmen eines streitigen Verfahrens entstehenden Gebühren liegen. Wird die gerichtsnahe Mediation nicht gesetzlich normiert, so muss die Ausgestaltung der Gebühren gleichzeitig auch wettbewerbsrechtlichen Aspekten Rechnung tragen und sollte sich daher an privatwirtschaftlichen Anbietern orientieren.

637 Empfehlenswert ist auch die Neuregelung der Verteilungsregelung des $\int 91$ ZPO, um - wie in anderen Ländern bereits üblich - Kostenanreize durch Kostensanktionen zu setzen. Die Kostenlastverteilung sollte sich dann nicht ausschließlich am Prozessausgang orientieren, sondern der obsiegenden Partei auch dann die Kosten - vollständig oder anteilig - auferlegen, wenn sie sich einem gerichtlich angeregten Versuch einer einvernehmlichen Konfliktbeilegung ungerechtfertigt widersetzt hat. Auf diesem Wege wären auch mittelbar Anreize für Rechtschutzversicherungen zur Änderung der Deckungsschutzzusage oder des Ausmaßes an Versicherungsschutz gegeben. Zumindest ist hier an eine verpflichtende Teilnahme an einer Informationssitzung zur gerichtsnahen Mediation zu denken.

638 Bei Einführung einer Gebührenordnung für die gerichtsnahe Mediation wird auch die Einführung einer der Prozesskostenhilfe vergleichbaren Mediationskostenhilfe erforderlich sein, um zu vermeiden, dass bestimmte Gruppen nicht von vornherein von der Möglichkeit der Inanspruchnahme der gerichtsnahen Mediation ausgeschlossen werden und um außerdem zu gewährleisten, dass die Justiz auch mit Blick auf das Angebot von gerichtsnaher Mediation der Sozialverantwortung des Staates gerecht wird. Um für die Parteien Anreize zur Nutzung des gerichtsnahen Mediationsangebotes zu setzen, könnte auch die Gewährung von Prozesskostenhilfe an das ernsthafte Bemühen um eine einvernehmliche Konfliktbeilegung geknüpft werden.

639 Hinsichtlich der prozessualen Ausgestaltung des gerichtsnahen Mediationsangebotes ist zu bedenken, dass - abgesehen von dem für den Mediator bestehenden Zeugnisverweigerungsrecht - die Vertraulichkeit des Mediationsverfahrens, insbesondere mit Blick auf einen nachfolgenden Prozess, nur durch Prozessvertrag und auch insoweit nicht vollständig gesichert werden kann. Um im nachfolgenden 
Prozess einen umfassenden Schutz der Vertraulichkeit aller vertraulich erörterter Umstände gewährleisten zu können, ist die gesetzliche Einführung eines entsprechenden umfassenden gesetzlichen Beweisverbotes zu empfehlen.

Ein positives Signal für die Konfliktparteien mit ebenfalls nur klarstellendem Charakter würde schließlich eine gesetzliche Regelung, die auch für Ausschlussfristen eine Fristhemmung für die Dauer von (Mediations-) Verhandlungen vorsieht, darstellen.

Zur Förderung der gerichtsnahen Mediation sind auf rechtspolitischer Ebene auch Verhandlungen mit den Rechtsschutzversicherern zu empfehlen, die auch für sie kostengünstige Alternative der (gerichtsnahen) Mediation unter bestimmten Voraussetzungen in ihr Leistungsprogramm aufzunehmen. Alternativ kann auch eine Änderung der Kostenverteilungsregelung in $\$ \int 91 \mathrm{ff}$. ZPO genügen, da damit mittelbar auch die entsprechenden Rechtsschutzversicherungsmöglichkeiten verändert werden.

Abschließend kann die gerichtsinterne Mediation als ein erfolgversprechender Weg hin zur einer größeren Entlastung der Justiz gelten - allerdings kann dies nur ein erster Schritt sein, der in einen größeren Rahmen gestellt werden muss, den diese Untersuchung nicht leisten konnte, für den aber die methodischen Grundlagen gelegt werden konnten. So wären die Chancen und die Ausgestaltung einer vorgerichtlichen Mediation, insbesondere der Anreizstrukturen für Parteien und Anwaltschaft, zu analysieren. Selbst für die gerichtsinterne Mediation ist das Bild noch unvollständig, da alternative Möglichkeiten für die Streitschlichtung bei einem bereits anhängigen Verfahren nicht in die Analyse einbezogen werden konnten, etwa die Beauftragung von Anwaltsmediatoren. Um ein geflügeltes Wort zu zitieren: „Much research has to be done“. 



\section{G. Literaturverzeichnis}

Aaken, Anne van, „Rational Choice“ in der Rechtswissenschaft, Zum Stellenwert der ökonomischen Theorie im Recht, Baden-Baden 2003.

Adams, Michael, Ökonomische Analyse des Zivilprozesses, Frankfurt a.M. 2002

Adams, Michael, Ökonomische Analyse des Zivilprozesses, Königsstein/Ts. 1981

Akerloff, George, A., The Market for "Lemons": Quality Uncertainty and the Market Mechanism, 84 Quarterly Journal of Economics 488 - 500 (1970)

Alexander, Janet, Judges'self-interest and procedural rules: Comment on Macey, 23 Journal of Legal Studies 647 (1994)

Allmayer-Beck, Joseph, Das österreichische Zivilrechts-Mediations-Gesetz, IDR 2001, 119 ff.

Alm-Merk, Heidi, Das Verhältnis zwischen Exekutive und der Verwaltungsgerichtsbarkeit sowie zu Inhalt und Grenzen der Mediation im Verwaltungs- und Verwaltungsgerichtsverfahren, NdsVBl. 1997, 245 ff.

Althammer, Christoph, Mediation als prozessuale Last, JZ 2006, 69 ff.

Arrow, Kenneth J., Methodological Individualism and Social Knowledge, 84 American Economic Review 1994, Papers and Proceedings, S. 1 ff.

Babusiaux, Ulrike, Richterliches Entscheidungsverhalten im Lichte von Rechtsdogmatik, Rechtssoziologie und Ökonomischer Analyse des Rechts, in: Schmidtchen, Dieter / Weth, Stephan (Hrsg.), Der Effizienz auf der Spur. Die Funktionsfähigkeit der Justiz im Lichte der ökonomischen Analyse des Rechts, Baden-Baden 1999, S. 157 ff.

Bamberger, Heinz Georg / Roth, Herbert (Hrsg.), Kommentar zum Bürgerlichen Gesetzbuch, Band 1, München 2003 (zitiert: Bearbeiter, in: Bamberger/Roth, BGB, Band 1)

Bargen, Joachim von, Mediation im Verwaltungsprozess - Eine neue Form konsensualer Konfliktlösung vor Gericht, DVBl 2004, 468 ff. 
Bauer, Günter / Maier, Kar / Stabl, Peter, Rechtsschutzversicherung, Kommentar zu den Allgemeinen Bedingungen für die Rechtsschutzversicherung (ARB 75 und ARB 94/2000) (begr. von Walter Harbauer), 7. Aufl., München 2004 (zitiert: Bearbeiter, in Harbauer)

Baumann, Antje, Förderung der Mediation durch das Gericht - Maßnahmen in England und Deutschland, IDR 2005, 9 ff.

Baumbach, Adolf / Hefermehl, Wolfgang, Wettbewerbsrecht, 23. Auflage, München 2004 (zitiert: Bearbeiter, in: Baumbach/Hefermehl, Wettbewerbsrecht)

Baumbach, Adolf / Lauterbach, Wolfgang / Albers, Jan / Hartmann, Peter, Zivilprozessordnung: mit Gerichtsverfassungsgesetz und anderen Nebengesetzen, 62. Auflage, München 2004 (zitiert: Bearbeiter, in: Baumbach/Lauterbach/Albers/P. Hartmann, ZPO)

Behrens, Peter, Aspekte einer ökonomischen Theorie des Rechts, RT 1981, S. $472 \mathrm{ff}$.

Behrens, Peter, Die Bedeutung der ökonomischen Analyse des Rechts für das Arbeitsrecht, ZfA 1989, S. 209 ff.

Benda, E. / Weber, A., Der Einfluß der Verfassung im Prozeßrecht, ZZP 96 (1983), $285 \mathrm{ff}$.

Berg-van der Wijk, Irma J., in: Blankenburg/Gottwald/Strempel (Hrsg.), Alternativen in der Ziviljustiz - Berichte, Analysen, Perspektiven, Köln 1982, S. 65 ff.

Bernstein, Lisa, Understanding the limits of court-connected ADR: A critique of federal court-annexed arbitration programs, 141 University of Pennsylvania Law Review 2169, 2195 (1993)

Bischof, Hans Helmut, RVG: Erste Gebührenprobleme für Schiedsverfahren und Mediation, SchiedsVZ 2004, $252 \mathrm{ff}$.

Böttcher, Bianca / Laskawy, Dirk Helge, Mediationsklauseln in Verträgen und Geschäftsbedingungen: Anregungen und Formulierungsvorschläge, Der Betrieb 2004, 1247 ff.

Boulle, Laurence / Nesic, Miryana, Mediation: Process, Principles, Practice, London u.a. 2001 
Braun, Anton, Gebührenabrechnung nach dem neuen Rechtsanwaltsvergütungsgesetz (RVG): Einführung mit Berechnungsbeispielen nach BRAGO und RVG-E im Vergleich, Recklinghausen 2004

Breidenbach, Stephan / Henssler, Martin (Hrsg.), Mediation für Juristen, Köln 1997

Breidenbach, Stephan, Mediation: Struktur, Chancen und Risiken von Vermittlung im Konflikt, Köln 1995, zugl. Habil.-Schr., München 1993/94

Brieske, Rembert, Haftungs- und Honorarfragen in der Mediation, in: Henssler, Martin / Koch, Ludwig (Hrsg.), Mediation in der Anwaltspraxis, Bonn 2000, S. 271 ff.

Bübring-Uble, Christian, Arbitration and Mediation in International Business.

Designing Procedures for Effective Conflict Management, Kluwer Law International, The Hague u.a. 1996

Bush, Robert A. Baruch / Folger, Joseph P., The Promise of Mediation: Responding to Conflict Through Empowerment and Recognition, San Francisco 1994

Canaris, Claus-Wilhelm, Die Feststellung von Lücken im Gesetz. Eine methodologische Studie über Voraussetzungen und Grenzen richterlicher Rechtsfortbildung praeter legem, 2. Auflage, Berlin 1983

Casper, Matthias / Risse, Jörg, Mediation von Beschlussmängelstreitigkeiten, ZIP 2000, 437 ff.

Chemnitz, Jürgen / Johnigk, Frank, Rechtsberatungsgesetz, 11. Auflage, Münster 2003

Cohen, Mark A., Explaining Judicial Behavior or What's "Unconstitutional" about the Sentencing Commission?, 7 Journal of Law, Economics, and Organization 183, 185 (1991)

Cohen, Mark A., The motives of judges: Empirical evidence from antitrust sentencing, 12 International Review of Law and Economics 12 (1992)

Coleman, James S., Foundations of Social Theory, Cambridge (Mass.), London 1990

Cooter, Robert D. / Rubinfeld, Daniel L., Economic Analysis of Legal Disputes and Their Resolution, 27 Journal of Economic Literature 1067, 1074 f. (1989) 
Coyle, Micheal, Defending the Weak and Fighting Unfairness: Can Mediators Respond to the Challenge?, 36 Osgoode Hall Law Journal (1998), $625 \mathrm{ff}$.

Davis, Albie M. / Salem, Richard A., Dealing with Power Imbalances in the Mediation of Interpersonal Disputes, 6 Mediation Quaterly (1984), $17 \mathrm{ff}$.

Deason, Ellen E., Procedural Rules for Complementary Systems of Litigation and Mediation Worldwide, Public Law and Legal Theory Working Paper Series No. 22/Center for Law, Policy and Social Science Working Paper Series No. 15, abrufbar unter http://ssrn.com/abstract $=583141$.

Diering, Björn / Timme, Hinnerk / Waschull, Dirk (Hrsg.), Sozialgesetzbuch X, Sozialverwaltungsverfahren und Sozialdatenschutz, Lehr- und Praxiskommentar, Baden-Baden 2004 (zitiert: Bearbeiter in LPK-SGB $\mathrm{X})$

Ditges, Thomas, Mediation und Rechtsstreit - ein Kosten- und Effizienzvergleich, IDR 2005, $74 \mathrm{ff}$.

Dreier, Horst (Hrsg.), Grundgesetz, Band 3: Art. 83 -146, Tübingen 2000 (zitiert: Bearbeiter, in: Dreier, GG, Band 3)

Dürschke, Joachim, Mediation - Ein Weg im sozialgerichtlichen Verfahren, SGb 2001, 532 ff.

Dütz, Wilhelm, Rechtsstaatlicher Gerichtsschutz im Privatrecht: Zum sachlichen Umfang der Zivilgerichtsbarkeit, Habil.-Schr., Münster 1970

Duve, Christian / Prause, Matthias, Mediation und Vertraulichkeit. Der Vorentwurf einer europäischen Mediationsrichtlinie, IDR 2004, 126 ff.

Duve, Christian, Brauchen wir ein Recht der Mediation? Zur Zukunft rechtlicher Rahmenregelungen für die Mediation in Deutschland und Europa, AnwBl 2004, 1 ff.

Duve, Christian, Mediation und Vergleich im Prozess. Eine Darstellung am Beispiel des Special Master in den USA, Köln 1999, zugl. Diss., München 1998

Duve, Christian, Rechtsberatung durch Mediatoren im Spiegel der Rechtsprechung. Zugleich eine Anmerkung zu LG Rostock, Urteil vom 11.8.2000, BB 2001, 692 ff. 
Eckardt, Bernd / Dendorfer, Renate, Der Mediator zwischen Vertraulichkeit und Zeugnispflicht - Schutz durch Prozessvertrag, MDR 2001, 786 ff.

Eidenmüller, Horst, Effizienz als Rechtsprinzip, Tübingen 1995

Eidenmüller, Horst, Lösungspaket schnüren und umsetzen, in: Duve, Christian / Eidenmüller, Horst / Hacke, Andreas, Mediation in der Wirtschaft. Wege zum professionellen Konfliktmanagement, Köln 2003

Eidenmüller, Horst, Verhandlungsmanagement durch Mediation, in: Henssler, Martin / Koch, Ludwig (Hrsg.), Mediation in der Anwaltspraxis, Bonn 2000, S. 39 ff.

Eidenmüller, Horst, Vertrags- und Verfahrensrecht der Wirtschaftsmediation: Mediationsvereinbarungen, Mediatorvertäge, Mediationsvergleiche, internationale Mediationsfälle, Köln 2001

Eidenmüller, Horst, Vertrags- und verfahrensrechtliche Grundfragen der Mediation: Möglichkeiten und Grenzen privatautonomen Konfliktmanagements, in: Breidenbach, Stephan / Coester-Waltjen, Dagmar / Heß, Burkhard / Nelle, Andreas / Wolf, Christian, Konsensuale Streitbeilegung. Akademisches Symposium zu Ehren von Peter F. Schlosser aus Anlass seines 65. Geburtstags, Bielefeld 2001, S. 45 ff.

Emmerich, Volker, Der unlautere Wettbewerb der öffentlichen Hand, Tübingen 1969

Enders, Horst-Reiner, Anwaltsgebühren rund um den Anwaltsvergleich - Teil I, JurBüro 1998, 337 ff.

Erichsen, Hans-Uwe / Ehlers, Dirk (Hrsg.), Allgemeines Verwaltungsrecht, 12. Auflage, Berlin 2002

Ewig, Eugen, Gesetzgebungsvorschlag der BRAK zu \278 ZPO (gerichtsnahe Mediation) - erarbeitet vom Ausschuss Mediation der BRAK, ZKM 2002, 45 ff.

Ferz, Sascha / Filler, Ewald, Mediation. Gesetzestexte und Kommentar, Wien 2003

Fiss, Owen, Against Settlement, 93 Yale Law Journal (1984), 1073 ff.

Fucik, Robert, Die Rolle des Richters in der ZPO. Eine ökonomische Analyse, in: Bundesministerium für Justiz/Lewisch/Rechberger, 100 Jahre ZPO: ökonomische Analyse des Zivilprozesses, 1998, S. 191, 193 
Fürst, Walther, Gesamtkommentar Öffentliches Dienstrecht, Band 1, Beamtenrecht des Bundes und der Länder, Richterrecht, Wehrrecht, Loseblatt, Berlin, Stand: 2004 (zitiert: Bearbeiter, in: Fürst, GKÖD, Band 1)

Gamm, Otto-Friedrich von, Verfassungs- und wettbewerbsrechtliche Grenzen des Wettbewerbs der öffentlichen Hand, WRP 1984, 303 ff.

Gamm, Ulrike / Patera, Mario, Das Tabu lebt! - Verfehlt Wirtschaftsmediation die Zielgruppe der österreichischen KMU?, IDR 2005, 59 ff.

Gerking, Wiebke, Die wirtschaftlichen Folgen langer Verfahrensdauer, in: Schmidtchen, Dieter / Weth, Stephan (Hrsg.), Der Effizienz auf der Spur. Die Funktionsfähigkeit der Justiz im Lichte der ökonomischen Analyse des Rechts, Baden-Baden 1999

Gern, Alfons, Zur Möglichkeit öffentlichrechtlicher Verträge zwischen Privaten, NJW 1979, 694 ff.

Gerold, Wilhelm / Schmidt, Herbert / Eicken, Kurt von / Madert, Wolfgang / Müller-Rabe, Steffen, Rechtsanwaltsvergütungsgesetz, 16. Auflage, München 2004 (zitiert: Bearbeiter, in: Gerold/H. Schmidt/v. Eicken/Madert/Müller-Rabe, RVG)

Gilson, Ronald J. / Mnookin, Robert H., Disputing through agents: Cooperation and conflict between lawyers in ligitation, 94 Columbia Law Review 509, 513 (1994)

Glenewinkel, Werner, Mediation als außergerichtliches Konfliktlösungsmodell: am Beispiel der Trennungs- und Scheidungsmediation in der Bundesrepublik Deutschland, Stuttgart 1999, zugl. Diss., Göttingen 1999

Göthlich, Stephan E., On the Nature of the Costs of Conflict, IDR 2005, $68 \mathrm{ff}$.

Gottwald, Walther, Alternative Streitbehandlungsformen: Erprobungsspielräume für gerichtsverbundene Modellversuche, AnwB1 2000, 265 ff.

Götz, von Olenhusen, Peter Götz, von, Gerichtsmediation - Richterliche Konfliktvermittlung im Wandel, ZKM 2004, $104 \mathrm{f}$.

Götz von Olenhusen, Peter Götz, von, Mediation durch Richter - ein Projekt mit Zukunft, DRiZ 2003, 396 f.

Gray, Owen V., Protecting the Confidentiality of Communications in Mediation, 36 Osgoode Hall Law Journal (1998), 667 ff. 
Greger, Reinhard / Engelhardt, Matthias, "Take mediation seriously or face the consequences", ZKM 2003, 4 ff.

Greger, Reinhard, Die Verzahnung von Mediation und Gerichtsverfahren in Deutschland, ZKM 2003, 240 ff.

Greger, Reinhard, Justizreform - und nun?, JZ 2002, 1020 ff.

Greger, Reinhard, Stand und Perspektiven der außergerichtlichen Streitbeilegung in Bayern, ZKM 2004, 196 ff.

Groth, Klaus-Martin / von Bubnoff, Daniela, Gibt es „gerichtsfeste“ Vertraulichkeit bei der Mediation?, NJW 2001, 338 ff.

Grupp, Klaus, Wirtschaftliche Betätigung der öffentlichen Hand unter dem Grundgesetz, ZHR 140 (1976), 367 ff.

Hacke, Andreas, Der ADR-Vertrag: Vertragsrecht und vertragliche Gestaltung der Mediation und anderer alternativer Konfliktlösungsverfahren, Heidelberg 2001, zugl. Diss., Münster 2001

Haffke, Bernhard, Legalität von Mediation im deutschen Rechtsraum, in: Duss-von Werdt, Josef / Mähler, Gisela / Mähler, Hans-Georg (Hrsg.), Mediation: Die andere Scheidung. Ein interdisziplinärer Überblick, Stuttgart 1995, S. 65 ff.

Hager, Günter, Konflikt und Konsens. Überlegungen zu Sinn, Erscheinung und Ordnung der alternativen Streitschlichtung, Tübingen 2001

Harte-Bavendamm, Henning / Henning-Bodewig, Frauke, Gesetz gegen den unlauteren Wettbewerb (UWG), München 2004 (zitiert: Bearbeiter, in: Harte-Bavendamm/Henning-Bodewig, UWG)

Hartmann, Christoph, Sicherung der Vertraulichkeit, in: Haft, Fritjof / von Schlieffen, Katharina (Hrsg.), Handbuch Mediation, München 2002, S. $712 \mathrm{ff}$.

Hartung, Wolfgang / Römermann, Volker, Praxiskommentar zum Rechtsanwaltsvergütungsgesetz, München 2004 (zitiert: Bearbeiter, in: Hartung/Römermann, RVG)

Hellriegel, Mathias, Mediation im Umweltrecht, Berlin 2002, zugl. Diss., Berlin 2001

Henssler, Martin / Prütting, Hanns, Bundesrechtsanwaltsordnung, 2. Auflage, München 2004 
Henssler, Martin, Mediation und Rechtsberatung, NJW 2003, 241 ff.

Heß, Burkhard / Sharma, Daniel, Rechtsgrundlagen der Mediation, in: Haft, Fritjof / von Schlieffen, Katharina (Hrsg.), Handbuch Mediation, München 2002, S. 675 ff.

Hoffmann-Riem, Wolfgang, Konfliktbewältigung in einer angebotsorientierten Rechtsschutzordnung, ZRP 1997, 190 ff.

Hoffmann-Riem, Wolfgang, Wahrheit, Gerechtigkeit, Unabhängigkeit und Effizienz - das Magische Viereck der Dritten Gewalt, JZ 1997, 1 ff.

Holznagel, Bernd / Ramsauer, Ulrich, Mediation im Verwaltungsrecht, in: Haft, Fritjof / Schlieffen, Katharina von, Handbuch Mediation, München 2002

Homann, Karl / Suchanek, Andreas, Ökonomik: Eine Einführung, Tübingen 2000

Hückstädt, Gerhard, Gerichtliche Mediation beim Landgericht Rostock - Ein Erfahrungsbericht, NJ 2005, 289 ff.

Huther, Edda, Gerichtsnahe Mediation aus der Sicht der Bayrischen Justiz, ZKM 2004, 247ff.

Isensee, Josef, Privatwirtschaftliche Expansion öffentlich-rechtlicher Versicherer, Der Betrieb 1979, 145 ff.

Jansen, Nicola, Die außergerichtliche obligatorische Streitschlichtung nach \ 15 a EGZPO, St. Augustin 2001, zugl. Diss., Bonn 2000/01

Janson, Gunnar, Ökonomische Theorie im Recht, Anwendbarkeit und Erkenntniswert im allgemeinen und am Beispiel des Arbeitsrechts, Ber$\operatorname{lin} 2004$

Kilian, Matthias, Erprobung der gerichtsnahen Mediation, ZKM 2005, 186 ff.

Kirchgässner, Gebhard, Homo Oeconomicus, 2. Aufl. Tübingen 2000

Kirchner, Christian, Ökonomische Theorie des Rechts, Berlin 1997

Kissel, Otto Rudolf / Mayer, Herbert, Gerichtsverfassungsgesetz, 4. Auflage, München 2005

Kloepfer, Michael, Verfahrensdauer und Verfassungsrecht. Verfassungsrechtliche Grenzen der Dauer von Gerichtsverfahren, JZ 1979, 209 ff. 
Klose, Alexander, Rechtliche Hürden auf dem Weg zur gerichtsinternen Mediation?, ZKM 2005, $146 \mathrm{ff}$.

Knack, Joachim (Begr.), Verwaltungsverfahrensgesetz, 7. Auflage, Köln u.a. 2000

Kobayashi, Bruce, H. / Parker, Jeffrey S., Civil Procedure: General, in:

Bouckaert/ de Geest: Encyclopedia of Law and Economics (http:/ / encyclo.findlaw.com/), 1999, 1 ff.

Koch, Ludwig, Vertragsgestaltung in der Mediation, in: Henssler, Martin /

Koch, Ludwig (Hrsg.), Mediation in der Anwaltspraxis, Bonn 2000, S. $245 \mathrm{ff}$.

Köhler, Helmut / Piper, Henning, Gesetz gegen den unlauteren Wettbewerb: mit Preisangabenverordnung, 3. Auflage, München 2002 (zitiert: Bearbeiter, in: Köhler/Piper, UWG)

Köper, Roman, Die Rolle des Rechts im Mediationsverfahren, Berlin 2003, zugl. Diss., Tübingen 2002

Köper, Roman, Eine Qualitätsstudie des Österreichischen ZivilrechtsMediations-Gesetzes, ZKM 2004, 161 ff.

Kopp, Ferdinand O. / Ramsauer, Ulrich, Verwaltungsverfahrensgesetz, 8. Auflage, München 2003

Kopp, Ferdinand O. / Schenke, Wolf-Rüdiger, Verwaltungsgerichtsordnung, 13. Auflage, München 2003

Kretschmer, Reinhard, Nichtanwaltliche Mediation und Rechtsberatungsgesetz, NJW 2003, 1500 ff.

Lande, John, Using Dispute Design Methods to Promote Good-Faith Participation in Court-Connected Medation Programs, 50 UCLA Law Review 69 (2002), 69 ff.

Landes, William M. / Posner, Richard A., Legal change, judicial behavior, and the diversity jurisdiction, 9 Journal of Legal Studies 367, 369 (1980)

Larenæ, Karl / Canaris, Claus-Wilhelm, Methodenlehre der Rechtswissenschaft, 3. Auflage, Berlin 1995

Layard, Peter R. /Walters, Alan A., Microeconomic Theory, New York 1978

Leibner, Wolfgang, Haftungsrechtliche Rahmenbedingungen für die Tätigkeit als Mediator, NJW 2002, $3521 \mathrm{ff}$. 
Löer, Lambert, Einbindung von Mediation in den Zivilprozess - Teil 1, ZKM 2005, $182 \mathrm{ff}$.

Löer, Lambert, Einbindung von Mediation in den Zivilprozess - Teil 2, ZKM 2006, 4 ff.

Löer, Lambert, Richterliche Mediation, ZZP 119 (2006), 199 ff.

Lörcher, Gino, Durchsetzbarkeit von Mediationsergebnissen, in: Haft, Fritjof / von Schlieffen, Katharina (Hrsg.), Handbuch Mediation, München 2002, S. $747 \mathrm{ff}$.

Luban, David, The Quality of Justice, 66 Denver University Law Review (1989), $381 \mathrm{ff}$.

Macey, Jonathan R., Judicial preferences, public choice, and the rules of procedure, 23 Journal of Legal Studies 627, 631 (1994)

Mäbler, Gisela / Mäbler, Hans-Georg, Kriterien für Gerechtigkeit in der Mediation, in: Dieter, Anne / Montada, Leo / Schulze, Annedore (Hrsg.), Gerechtigkeit im Konfliktmanagement und in der Mediation, Frankfurt/New York 2000, S. 9 ff.

Mäbler, Gisela / Mäbler, Hans-Georg, Mediation im Spektrum außergerichtlicher Konfliktbeilegung, AnwBl. 1997, 535 ff.

Mäbler, Gisela / Mäbler, Hans-Georg, Missbrauch von in der Mediation erlangten Informationen, ZKM 2001, 4 ff.

Mangoldt, Hermann von / Klein, Friedrich / Starck, Christian, Das Bonner Grundgesetz, Band 1, 4. Auflage, München 1999 (zitiert: Bearbeiter, in: v. Mangoldt/Klein/Starck, GG, Band 1)

Mangoldt, Hermann von / Klein, Friedrich / Stark, Christian, Das Bonner Grundgesetz, Band 3, 4. Auflage, München 2001 (zitiert: Bearbeiter, in: v. Mangoldt/Klein/Starck, GG, Band 3)

Mankowski, Peter, Ablehnung eines Mediationsnagebots und Prozesskostenhilferecht - Anm. zu OLG Hamm v. 20.3.2003 - 3 WF 44/03 und AG Bochum v. 20.12.2002 - 59 F 335/02, ZKM 2003, $197 \mathrm{ff}$.

Mankowski, Peter, Anmerkung zu OLG Rostock, Urteil vom 20.6.2001, MDR 2001, $1198 \mathrm{ff}$.

Mankowski, Peter, Gibt es gesetzliche Kostenanreize zur Mediation bereits unter geltendem Recht? ZKM 2004, 8 ff. 
Martiensen, Jörn, Institutionenökonomik - Die Analyse der Bedeutung von Regeln und Organisationen für die Effizienz ökonomischer Tauschbeziehungen, München 2000

Maurer, Hartmut, Allgemeines Verwaltungsrecht, 14. Auflage, München 2002

Mayer, Bernard, The Dynamics of Power in Mediation and Negotiation, 16 Mediation Quaterly (1987), $75 \mathrm{ff}$.

Mayer, Hans Jochen / Kroiß, Ludwig (Hrsg.), Rechtsanwaltsvergütungsgesetz, Baden Baden 2004 (zitiert: Bearbeiter, in: Mayer/Kroiß, RVG)

Medicus, Dieter, Schuldrecht: ein Studienbuch, Band 2, Besonderer Teil, 12. Auflage, München 2004

Menkel-Meadow, Carrie, Pursuing Settlement in an Adversary Culture: A Tale of Innovation Co-opted or "The Law of ADR", 19 Florida State University Law Review (1991), 1 ff.

Meyer-Ladewig, Jens, Sozialgerichtsgesetz, 7. Auflage, München 2002

Miller, Geoffrey P., Some agency problems in settlement, 16 Journal of Legal Studies 189, 209 f. (1987)

Mnookin, Robert. H. / Kornhauser, Lewis., Bargaining in the shadow of the law, 88 (5) Yale Law Journal 950 ff. (1979)

Monßen, Hans-Georg, Bringt die ZPO-Reform den Durchbruch für die Mediation?, ZKM 2003, $116 \mathrm{ff}$.

Monßen, Hans-Georg, Mediation - nur noch Anwaltssache? Das Rechtsberatungsgesetz als Hüter des Status Quo und Instrument, nicht anwaltlichen Mediatoren den Marktzutritt zu erschweren - Anmerkung zu LG Rostock, Urteil vom 11.8.2000, AnwBl 2001, 169 f.

Münchener Kommentar ₹um Bürgerlichen Geset乏buch, Band 1, 4. Auflage, München 2001 (zitiert: Bearbeiter, in: MünchKommBGB, Band 1)

Münchener Kommentar zum Bürgerlichen Gesetzbuch, Band 4, 3. Auflage, München 1997 (zitiert: Bearbeiter, in: MünchKommBGB, Band 4)

Münchener Kommentar zum Bürgerlichen Gesetz̧buch, Band 5, 4. Auflage, München 2004 (zitiert: Bearbeiter, in: MünchKommBGB, Band 5)

Münchener Kommentar zur Zivilprozessordnung: mit Gerichtsverfassungsgesetz. und Nebengesetzen, Band 1, 2. Auflage, München 2000 (zitiert: Bearbeiter, in: MünchKommZPO, Band1) 
Münchener Kommentar zur Zivilprozessordnung: mit Gerichtsverfassungsgesetz, und Nebengesetzen, Band 2, 2. Auflage, München 2000 (zitiert: Bearbeiter, in: MünchKommZPO, Band 2)

Münchener Kommentar zur Zivilprozessordnung: mit Gerichtsverfassungsgesetz. und Nebengesetzen, Band 3, 2. Auflage, München 2001 (zitiert: Bearbeiter, in: MünchKommZPO, Band 2)

Musielak, Hans-Joachim, Kommentar zur Zivilprozessordnung, 3. Auflage, München 2002 (zitiert: Bearbeiter, in: Musielak, ZPO)

Nerlich, Jörg, Außergerichtliche Streitbeilegung mittels Anwaltsvergleichs, MDR 1997, $416 \mathrm{ff}$.

Newmark, Christopher, Agree to mediate ... or face the consequences - A review of the English courts' approach to mediation, SchiedsVZ 2003, $23 \mathrm{ff}$.

Nölting, Hubertus, Die Haftung von Mediatoren, ZKM 2004, 231 ff.

Nölting, Hubertus, Mediatorenverträge, Köln 2003

North, Douglass C., Economic Performance Through Time, 84 American Economic Review 359 (1994)

Oberhammer, Paul / Domej, Tanja, Ein rechtlicher Rahmen für die Mediation in Österreich. Zum neuen Zivilrechts-Mediations-Gesetz, ZKM 2003, $144 \mathrm{f}$.

Ortloff, Karsten-Michael, Mediation außerhalb und innerhalb des Verwaltungsprozesses, NVwZ 2004, 385 ff.

Ortloff, Karsten-Michael, Kommunikationsdefizite im Verwaltungsprozess?, NVwZ 2002, 1310 ff.

Ortloff, Karsten-Michael, Mediation und Verwaltungsprozess, in: Haft, Fritjof / von Schlieffen, Katharina (Hrsg.), Handbuch Mediation, München 2002, S. 762 ff.

Palandt, Otto (Hrsg.), Bürgerliches Gesetzbuch : mit Einführungsgesetz (Auszug), BGB-Informationspflichten-Verordnung, Unterlassungsklagengesetz, Produkthaftungsgesetz, Erbbaurechtsverordnung, Wohnungseigentumsgesetz, Hausratsverordnung, Lebenspartnerschaftsgesetz, Gewaltschutzgesetz (Auszug), 63. Auflage, München 2004 (zitiert: Bearbeiter, in: Palandt, BGB) 
Pawlowski, Hans-Martin, Einführung in die Juristische Methodenlehre, 2. Auflage, Heidelberg 2000.

Pearson, Jessica, An Evaluation of Alternatives to Court Adjudication, 7 Justice System Journal (1982), 420 ff.

Piper, Henning, Zum Wettbewerb der öffentlichen Hand, GRUR 1986, 574 ff.

Pitkowits, Niklaus, Der Mediations-Richtlinienvorschlag der EU: Gleichstellung der Mediation mit Gerichtsverfahren!, ZKM 2005, 68 ff.

Pitschas, Rainer, Mediation als Methode und Instrument der Konfliktmittlung im öffentlichen Sektor, NVwZ 2004, 396 ff.

Ponschab, Reiner, Mediation in der anwaltlichen Tätigkeit, in: Breidenbach, Stephan / Henssler, Martin (Hrsg.), Mediation für Juristen, Köln 1997, S. 93 ff.

Posner, Richard A., An economic approach to legal procedure and judicial administration, 2 Journal of Legal Studies 399, 420 (1973)

Posner, Richard A., Economic Analysis of Law, 5. Aufl. New York 1998

Posner, Richard A., What do judges maximize? (The same thing everybody else does), Chicago Working Paper in Law \& Economics No. 15 (2nd series)

Preibisch, Wolfgang, Außergerichtliche Vorverfahren in Streitigkeiten der Zivilgerichtsbarkeit, Berlin 1982, zugl. Diss., Berlin 1980

Pruckner, Martina, Recht der Mediation. ZivMediatG mit Kurzkommentar, Vertrags- und Berufsrecht, Arbeiten auf EU-Ebene, Wien 2003

Prütting, Hanns (Hrsg.), Außergerichtliche Streitbeilegung. Handbuch für die Praxis, München 2003

Prütting, Hanns, Haftung, in: Haft, Fritjof / von Schlieffen, Katharina (Hrsg.), Handbuch Mediation, München 2002, S. 824 ff.

Pryles, M., Assessing Dispute Resolution Procedures, 7 The American Review of International Arbitration (1996), 267 ff.

Rennen, Günter / Caliebe, Gabriele, Rechtsberatungsgesetz, 3. Auflage, München 2001

Richter, Rudolf / Furubotn, Eirik, Neue Institutionenökonomik, 3. Auflage, Tübingen 2003 
Riehle, Eckart, Rechtsberatungsgesetz und Mediation nach \$S 17, 28 SGB VII, ZfJ 2001, 13 ff.

Risse, Jörg / Wagner, Christof, Mediation im Wirtschaftsrecht, in: Haft, Fritjof / von Schlieffen, Katharina (Hrsg.), Handbuch Mediation, München 2002, S. 987 ff.

Risse, Jörg, Die Rolle des Rechts in der Wirtschaftsmediation, Beilage Nr. 9 zu BB 1999, 1 ff.

Risse, Jörg, Wirtschaftsmediation, NJW 2000, 1614 ff.

Ritter, Ernst-Hasso, Justiz - verspätete Gewalt in der Wettbewerbsgesellschaft, NJW 2001, 3440 ff.

Roth, Marianne, The Proposal for an EU Directive on Certain Aspects of Mediation in Comparison with the Austrian Mediation law, IDR 2005, 114 ff.

Rothschild, Michael / Stiglits, Joseph, Equilibrium in Competitive Insurance Markets: An Essay on the Economics of Imperfect Information, 90 Quarterly Journal of Economics 629 - 649 (1976)

Schäfer, Hans-Bernd / Ott, Claus, Lehrbuch der ökonomischen Analyse des Zivilrechts, 3. Aufl., Berlin u.a. 2000

Schlette, Volker, Die Verwaltung als Vertragspartner: Empirie und Dogmatik verwaltungsrechtlicher Vereinbarunen zwischen Behörde und Bürger, Tübingen 2000, zugl. Habil.-Schr., Göttingen 1999

Schlosser, Peter, Einverständliches Parteihandeln im Zivilprozeß, Tübingen 1968

Schmidt, Reiner, Öffentliches Wirtschaftsrecht - Allgemeiner Teil, Berlin 1990

Schmidtchen, Dieter / Kirstein, Roland, Prozeßkostenreform und richterliche Entdeckungsfähigkeit, in: Schmidtchen, Dieter / Weth, Stephan (Hrsg.), Der Effizienz auf der Spur. Die Funktionsfähigkeit der Justiz im Lichte der ökonomischen Analyse des Rechts, Baden-Baden 1999, S. $207 \mathrm{ff}$.

Schmidtchen, Dieter / Weth, Stephan, Ökonomische Analyse des Rechts: Ein Ausweg aus der Krise der Justiz, in: Schmidtchen, Dieter / Weth, Stephan (Hrsg.), Der Effizienz auf der Spur. Die Funktionsfähigkeit der Justiz im Lichte der ökonomischen Analyse des Rechts, BadenBaden 1999, S. 237 ff. 
Schmidtchen, Dieter, Die ökonomische Analyse des Rechts, in: Schmidtchen, Dieter / Weth, Stephan (Hrsg.), Der Effizienz auf der Spur. Die Funktionsfähigkeit der Justiz im Lichte der ökonomischen Analyse des Rechts, Baden-Baden 1999, S. 9 ff.

Schmidt-Räntsch, Günther, Deutsches Richtergesetz: Richterwahlgesetz, 5. Auflage, München 1995 (zitiert: Schmidt-Räntsch, DRiG)

Schneider, Norbert / Mock, Peter, Das neue Gebührenrecht für Anwälte. Abrechnen nach dem neuen RVG, Bonn 2004

Schoch, Friedrich / Schmidt-Aßmann, Eberhard / Piet₹ner, Rainer, Verwaltungsgerichtsordnung, Band 2, Loseblatt, München, Stand: September 2003 (zitiert: Bearbeiter, in: Schoch/Schmidt-Aßmann/Pietzner, VwGO)

Schreiber, Frank, Wie „gerichtsnah“ kann Mediation funktionieren? Die Rollen von Richtern und Mediatoren müssen deutlich getrennt werden, Betrifft Justiz 2004, 216 ff.

Schricker, Helmut, Wirtschaftliche Tätigkeit der öffentlichen Hand und unlauterer Wettbewerb, 2. Auflage, Köln u.a. 1987

Schumann, Jochen / Meyer, Ulrich / Ströbele, Wolfgang, Grundzüge der mikroökonomischen Theorie, 7. Auflage, Berlin u.a. 1999

Scbünemann, Wolfgang B., Wettbewerbsrechtliche Zulässigkeit wirtschaftlicher Tätigkeit der öffentlichen Hand, in: Stober, Rolf / Vogel, Hanspeter (Hrsg.), Wirtschaftliche Betätigung der öffentlichen Hand. Staat und Kommunen als Konkurrent der Privatwirtschaft, Köln u.a. 2000

Schwab, Karl Heinz / Gottwald, Peter, Verfassung und Zivilprozeß, Bielefeld 1984

Schwintowski, Hans-Peter, Ökonomische Theorie des Rechts, JZ 1998, 581 ff.

Smith, Janet / Cox, Steven R., The pricing of legal services: A contractual solution to the problem of bilateral opportunism, 14 Journal of Legal Studies 167, 169 ff. (1985)

Sobich, Philip, Neuere Entwicklungen im englischen Zivilprozessrecht - Die ersten Jahre nach „Woolf“, ZVglRWiss 103 (2004), 69 ff.

Soergel, Hans Theodor (Begr.) / Siebert, Wolfgang (Hrsg.), Bürgerliches Gesetzbuch: nebst Einführungsgesetz, Band 4/2, 12. Auflage, Stuttgart u.a. 1999 (zitiert: Bearbeiter, in: Soergel, BGB, Band 4/2) 
Sommer, Herbert / Konert, Karl-Hans / Sommer, Imke, Niedersächsisches Beamtengesetz, Frankfurt a.M. 2001 (zitiert: Sommer/Konert/Sommer, $\mathrm{NBG})$

Spellbrink, Wolfgang, Mediation im sozialgerichtlichen Verfahren - Ein Zwischenruf, SGb 2003, 141 ff.

Spindler, Gerald / Apel, Katharina / Spalckhaver, Judith, Rechtsökonomische Grundlagen der gerichtsnahen Mediation, ZKM 2003, 192 ff.

Staudinger, Julius von (Begr.) / Beitzke, Günther (Hrsg.), Julius von Staudingers Kommentar zum Bürgerlichen Gesetzbuch: mit Einführungsgesetz und Nebengesetzen, S\$ 652-704, Berlin, 13. Bearbeitung, 1995 (zitiert: Bearbeiter, in: Staudinger, BGB, $\int \$$ 652-704)

Staudinger, Julius von (Begr.) / Beitzke, Günther (Hrsg.), Julius von Staudingers Kommentar zum Bürgerlichen Gesetzbuch: mit Einführungsgesetz und Nebengesetzen, SS 164-240, Berlin, Neubearbeitung 2004 (zitiert: Bearbeiter, in: Staudinger, BGB, $\int S \int S$ 164-240)

Staudinger, Julius von (Begr.) / Beit₹ke, Günther (Hrsg.), Julius von Staudingers Kommentar zum Bürgerlichen Gesetzbuch: mit Einführungsgesetz und Nebengesetzen, S\$241-243, Berlin, 13. Bearbeitung 1995 (zitiert: Bearbeiter, in: Staudinger, BGB, JS 241-243)

Stein, Friedrich / Jonas, Martin / Berger, Christian, Kommentar zur Zivilprozessordnung, Band 9, 22. Auflage, Tübingen 2002 (zitiert: Bearbeiter, in: Stein/Jonas, ZPO, Band 9)

Stein, Friedrich / Jonas, Martin / Berger, Christian, Kommentar zur Zivilprozessordnung, Band 2, 21. Auflage, Tübingen 1994 (zitiert: Bearbeiter, in: Stein/Jonas, ZPO, Band 2)

Stein, Friedrich / Jonas, Martin / Berger, Christian, Kommentar zur Zivilprozessordnung, Band 3, 21. Auflage, Tübingen 1999 (zitiert: Bearbeiter, in: Stein/Jonas, ZPO, Band 3)

Stein, Friedrich / Jonas, Martin / Berger, Christian, Kommentar zur Zivilprozessordnung, Band 4/2, 21. Auflage, Tübingen 1999 (zitiert: Bearbeiter, in: Stein/Jonas, ZPO, Band 4/2)

Stein, Friedrich / Jonas, Martin / Berger, Christian, Kommentar zur Zivilprozessordnung, Band 6, 21. Auflage, Tübingen 1995 (zitiert: Bearbeiter, in: Stein/Jonas, ZPO, Band 6) 
Stelkens, Paul / Bonk, Heinz Joachim / Sachs, Michael, Verwaltungsverfahrensgesetz, 6. Auflage, München 2001

Stobbe, Alfred, Mikroökonomik, 2. Auflage, Berlin 1991

Stober, Rolf, Rein gewerbliche Betätigung der öffentlichen Hand und Verfassung, ZHR 145 (1981), 565 ff.

Thomas, Heinz / Putæo, Hans, Zivilprozessordnung: mit Gerichtsverfassungsgesetz, den Einführungsgesetzen und europarechtlichen Vorschriften, 26. Auflage, München 2004 (zitiert: Bearbeiter, in: Thomas/Putzo, ZPO)

Tyler, Tom R., The Quality of Dispute Resolution Procedures and Outcomes: Measurement Problems and Possibilities, 66 Denver University Law Review (1989), 419 ff.

Ule, Carl Hermann / Laubinger, Hans-Werner, Verwaltungsverfahrensrecht. Ein Lehrbuch für Studium und Praxis, 4. Auflage, Köln u.a. 1995

Voßkuble, Andreas, Rechtsschutz gegen Richter: zur Integration der Dritten Gewalt in das verfassungsrechtliche Kontrollsystem vor dem Hintergrund des Art. 19 Abs. 4 GG, München 1993 (zitiert: Voßkuhle, Rechtsschutz gegen Richter)

Wagner, Gerhard, Alternative Streitbeilegung und Verjährung, NJW 2001, 182 ff.

Wagner, Gerhard, Das neue Verjährungsrecht - Ein Segen für die außergerichtliche Streitbeilegung, ZKM 2002, 103 ff.

Wagner, Gerhard, Gerichtlich veranlasste Mediation: England als Vorreiter, ZKM 2004, 100 ff.

Wagner, Gerhard, Prozeßverträge: Privatautonomie im Verfahrensrecht, Tübingen 1998

Wagner, Gerhard, Sicherung der Vertraulichkeit von Mediationsverfahren durch Vertrag, NJW 2001, 1398 ff.

Wagner,Klaus-R., Alternative Streitvermeindung: Notarielle Beurkundung, Betreuung, Schlichtung, BB 1997, 53 ff.

Walther, Harald, Mediation durch Verwaltungsgerichte, ZKM 2005, 53 ff.

Wasilewski, Rainer, Streitverhütung durch Rechtsanwälte : empirische Untersuchung von Umfang, Struktur und Bedingungen außergerichtlicher 
Beilegung zivilrechtlicher Streitigkeiten durch Rechtsanwälte, Köln, Essen 1990

Watts, Alison, Bargaining through an Expert Attorney, 10 Journal of Law, Economics and Organization (1994), 168 ff.

Wegmann, Bernd, Der Vertrag über die außergerichtliche Streitbeilegung im Konfliktfall, Zeitschrift für Mediation 1999, 146 ff.

Wegmann, Bernd, Die vorsorgende Verabredung außergerichtlicher Streitbeilegung im Zivilrecht, Zeitschrift für Mediation 1999, 80 ff.

Weigand, Frank Bernd, Alternative Streiterledigung. „Alternative Dispute Resolution“ auch in Deutschland?, BB 1996, 2106 ff.

Weigel, Wolfgang, Rechtsökonomik, München 2003

Weigel, Wolfgang, Zivilrechtliche Absicherung von Mediationsübereinkommen, eine Erkundung, in: Schäfer, Hans-Bernd / Lwowski, HansJürgen (Hrsg.), Konsequenzen wirtschaftsrechtlicher Normen. Kreditrecht - Verbraucherschutz - Allgemeines Wirtschaftsrecht, Festschrift für Claus Ott zum 65. Geburtstag, Wiesbaden 2002, S. 491 ff. (zitiert: Weigel, in: FS Ott)

Wein, Thomas, Recht durch Rechtsanwälte? Eine ökonomische Analyse des

Marktes für Rechtsanwaltsdienstleistungen, Berlin 1995

Wieczorek, Bernhard / Schütre, Rolf A., Zivilprozessordnung und Nebengesetze, Großkommentar, Erster Band (Einleitung; \$S 1 - 127a ZPO), 2. Teilband (\$S 50 bis 127 a) 3. Auflage, Berlin, New York, 1994 (zitiert: Bearbeiter, in Wieczorek/Schütze, ZPO)

Wolf, Christian, Die institutionelle Handelsschiedsgerichtsbarkeit, München 1992, zugl. Diss., München 1991

Wolf, Manfred, Richter und Rechtspfleger im Zivilverfahren, ZZP 99 (1986), $361 \mathrm{ff}$.

Wulffen, Matthias von, Sozialgesetzbuch, Zehntes Buch Sozialgesetzbuch Sozialverwaltungsverfahren und Sozialdatenschutz - (SGB X), 4 Aufl., München 2001 (zitiert: Bearbeiter, in von Wulffen, SGBX)

Ziekow, Jan, Mediation in der Verwaltungsgerichtsbarkeit. Möglichkeiten der Implementation und rechtliche Folgerungen, NVwZ 2004, 390 ff. 
Zöller, Richard (Begr.), Zivilprozessordnung: mit Gerichtsverfassungsgesetz und den Einführungsgesetzen, mit Internationalem Zivilprozessrecht, EG-Verordnungen, Kostenamerkungen, 24. Auflage, München 2004 (zitiert: Bearbeiter, in: Zöller, ZPO) 